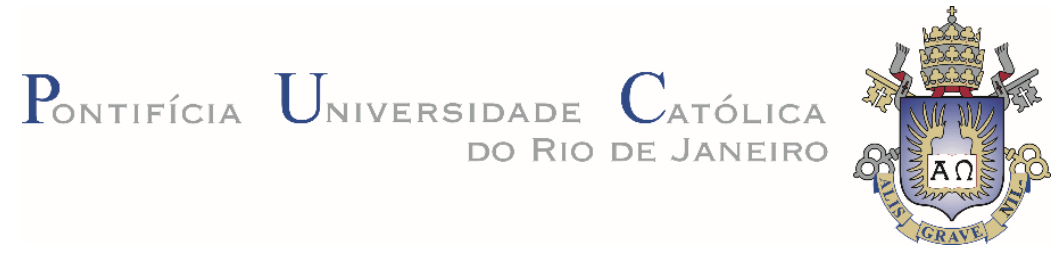

Leonardo dos Santos Lourenço Bastos

\title{
An essay on analysis of performance and use of resources in healthcare systems
}

Tese de Doutorado

Thesis presented to the Programa de Pós-graduação em Engenharia de Produção of PUC-Rio in partial fulfillment of the requirements for the degree of Doutor em Engenharia de Produção.

Advisor: Prof. Silvio Hamacher Co-advisor: Prof. Fernando Augusto Bozza 


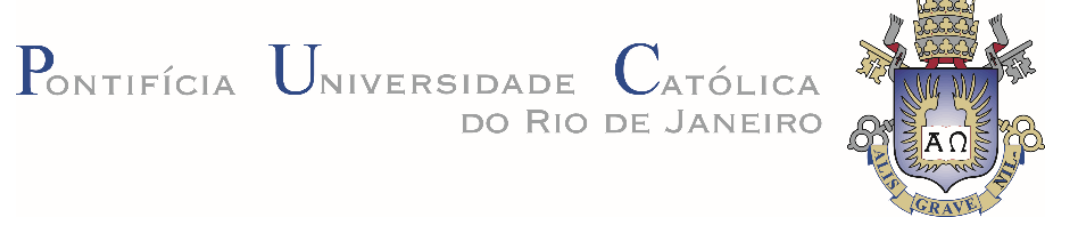

\section{Leonardo dos Santos Lourenço Bastos}

\section{An essay on analysis of performance and use of resources in healthcare systems}

Thesis presented to the Programa de Pós-graduação em Engenharia de Produção of PUC-Rio in partial fulfillment of the requirements for the degree of Doutor em Engenharia de Produção. Approved by the Examination Committee:

Prof. Silvio Hamacher Advisor Departamento de Engenharia Industrial - PUC-Rio

Prof. Fernando Augusto Bozza Co-advisor Fundação Oswaldo Cruz - FIOCRUZ

Prof. Paula Medina Maçaira Louro Departamento de Engenharia Industrial - PUC-Rio

Prof. Otavio Tavares Ranzani Faculdade de Medicina - FMUSP

Prof. Jorge Ibrain Figueira Salluh Instituto D'Or de Ensino e Pesquisa - IDOR

Prof. Marcio Soares Instituto D'Or de Ensino e Pesquisa - IDOR 
All rights reserved.

\section{Leonardo dos Santos Lourenço Bastos}

Graduated in Production Engineering at the State University of Para in 2015 and obtained his M.Sc. Degree in Production Engineering from the Pontifical Catholic University of Rio de Janeiro in 2018.

Bibliographic data

Bastos, Leonardo dos Santos Lourenço

An essay on analysis of performance and use of resources in healthcare systems / Leonardo dos Santos Lourenço Bastos; advisor: Silvio Hamacher; co-advisor: Fernando Augusto Bozza. - 2021.

221 f. : il. color. ; $30 \mathrm{~cm}$

Tese (doutorado)-Pontifícia Universidade Católica do Rio de Janeiro, Departamento de Engenharia Industrial, 2021.

Inclui bibliografia

1. Engenharia Industrial - Teses. 2. Sistemas de saúde. 3. Recursos de saúde. 4. Análise de desempenho. 5. Desfechos. 6. COVID-19. I. Hamacher, Silvio. II. Bozza, Fernando Augusto. III. Pontifícia Universidade Católica do Rio de Janeiro. Departamento de Engenharia Industrial. IV. Título. 
To my parents, Carlos and Mônica, my sister, Isabela, and my nephew, Carlos Eduardo. 


\section{Acknowledgments}

To God for all the strength, bless and guidance that could assist me towards my objectives.

To my parents, Carlos and Monica, my sister Isabela and my nephew Carlos Eduardo, my grandmother Elizete, my aunts, uncles, and cousins, for the love and support always present in my journeys.

To Verissa, for all the love, support, friendship, and motivation given to me towards my dreams and objectives, even in the hardest of the moments.

To my longtime friends, for the constant partnership and support. Despite the distance we are always close somehow and this really motivates me.

To my new friends from this recent journey, for introducing me to new universes and sharing your precious lessons. We have grown together, and I wish you all the best.

To my advisors, Silvio Hamacher and Fernando Bozza. You have opened great opportunities for improving professionally and as a person. Thank you for all the learning, lessons, brainstorms, and opportunities given, which certainly assisted the development of this and other projects.

To the researchers and colleagues that offered me the opportunity to contribute, to discuss, and learn together. A special thanks for all friends and colleagues from the healthcare world: Otavio, Pedro, Jorge, Marcio, Zampieri. You have taught me new perspectives, from which I am very grateful.

To all the Professors, colleagues, and employees at DEI, for providing the assistance and the necessary resources that conveyed the development of this dissertation and myself

To CNPq and PUC-Rio, for providing adequate resources and the opportunity to participate in this great university.

This study was financed in part by the Coordenação de Aperfeiçoaento de Pessoal de Nível Superior - Brasil (CAPES) - Finance Code 001 


\section{Abstract}

Bastos, Leonardo dos Santos Lourenço; Hamacher, Silvio (Advisor); Bozza, Fernando (Co-Advisor). An essay on analysis of performance and use of resources in healthcare systems. Rio de Janeiro, 2021. 221p. Tese de Doutorado - Departamento de Engenharia Industrial, Pontifícia Universidade Católica do Rio de Janeiro.

The adequate management of healthcare resources is essential to provide optimal patient care, especially under high stress/strain conditions or limited resources. Benchmarking is helpful to evaluate the performance and quality of care within these systems and provide targets for improvement. This is especially important for the intensive care units (ICUs), which deal with complex cases, high costs and provides relevant insights for treating severe diseases. Under usual conditions, assessing performance in intensive care is complex since metrics must account for the patient's case-mix and the unit's organizational settings. When high strain or stress conditions arise, the resource use increases, and the unit performs in unusual conditions. One of these settings is the COVID-19 pandemic, which has overwhelmed healthcare systems worldwide since December 2019, notably intensive care resources. This thesis aims to evaluate the use of resources and performance of healthcare systems under the perspectives of before and during the COVID-19 pandemic. Using data from Brazilian hospitals, we developed six individual studies aiming to perform ICU benchmarking in a pre-pandemic period and understand the use of ICU resources and outcomes during the progression of the pandemic. We managed each work as data science projects following the Data Science Life Cycle, under the Design Science research framework, and used different statistical approaches to analyze data. Our main results show that before the pandemic, the assessment of quality-of-care metrics and active surveillance of infections were associated with efficient ICU units. During the pandemic period, the use of resources and outcomes varied temporally and regionally in Brazil. North and Northeast, regions with more vulnerable healthcare systems, showed poor outcomes and lower availability of ICU resources than South and Southeast regions. The impact on the Brazilian healthcare system was intensified in a second pandemic surge, showing increasing use of respiratory resources and mortality. Finally, when evaluating the mortality evolution in a 
network of private hospitals that underwent preparedness and presented large availability of resources, the overall mortality was low and decreased over time. Noninvasive respiratory support was independently associated with a reduction in mortality

\section{Keywords}

Healthcare systems; Resource management; Benchmarking; Outcomes; COVID-19 


\section{Resumo}

Bastos, Leonardo dos Santos Lourenço; Hamacher, Silvio; Bozza, Fernando. Um ensaio sobre análise de desempenho e uso de recursos em sistemas de saúde. Rio de Janeiro, 2021. 221p. Tese de Doutorado - Departamento de Engenharia Industrial, Pontifícia Universidade Católica do Rio de Janeiro.

A gestão adequada dos recursos de saúde é essencial para fornecer os cuidados ideais aos pacientes, especialmente em condições de alta pressão no sistema ou recursos limitados. O benchmarking é útil para avaliar o desempenho e a qualidade do atendimento em sistemas de saúde e para identificar pontos de melhoria. Na unidade de terapia intensiva (UTI) isto é essencial, pois nela há ocorrência de casos complexos, custos elevados e geração de conhecimento relevante para o tratamento de doenças graves. Em condições normais, a avaliação do desempenho em terapia intensiva é uma tarefa difícil, uma vez que métricas de desempenho devem levar em conta o perfil dos paciente admitidos e os aspectos organizacionais da unidade. Condições de alta pressão no sistema aumentam a variabilidade no uso de recursos e, portanto, a unidade passa desempenhar de maneira incomum. Um desses cenários é a pandemia COVID-19, que sobrecarregou os sistemas de saúde pelo mundo desde dezembro de 2019, especialmente em termos da terapia intensiva. Esta tese tem como objetivo avaliar a utilização de recursos e o desempenho dos sistemas de saúde nos cenários de pré e durante a pandemia de COVID19. Utilizando dados de hospitais brasileiros, desenvolvemos seis estudos individuais com o objetivo de realizar benchmarking em UTIs em um período pré-pandêmico e compreender o uso de recursos e resultados da unidade durante a progressão da pandemia. Cada trabalho foi executado como um projetosde Ciência de Dados seguindo o Ciclo de Vida da Ciência de Dados, embasado pelo Design Science framework, e usando diferentes técnicas e modelagens estatísticas para analisar dados. Os principais resultados mostraram que antes da pandemia os dados de UTIs brasileiras indicavam que a avaliação das métricas de qualidade e a vigilância ativa de infecções eram atividade organizacionais associadas às unidades eficientes. Além disso, durante o período de pandemia o uso de recursos e os desfechos variaram temporal e regionalmente no Brasil. Regiões com sistemas de saúde mais vulneráveis e menor disponibilidade de recursos de UTI, tais como Norte e Nordeste, apresentaram panoramas desfavoráveis em comparação às 
regiões Sul e Sudeste. Este impacto no sistema de saúde brasileiro foi intensificado em um segundo surto da pandemia, mostrando aumento no uso de recursos respiratórios e mortalidade. Por fim, ao avaliar uma rede de hospitais privados com grande disponibilidade de recursos, verificamos que a mortalidade geral foi baixa, e o suporte respiratório não invasivo foi independentemente associado à redução da mortalidade.

\section{Palavras-chave}

Sistemas de saúde; Recursos de saúde; Análise de desempenho; Desfechos; COVID-19 


\section{Summary}

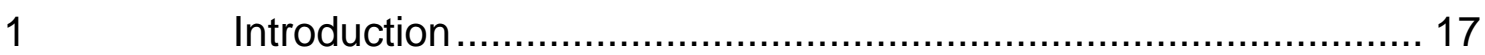

$1.1 \quad$ Research topics and objectives ............................................... 19

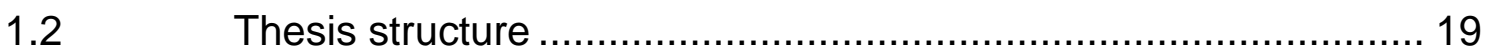

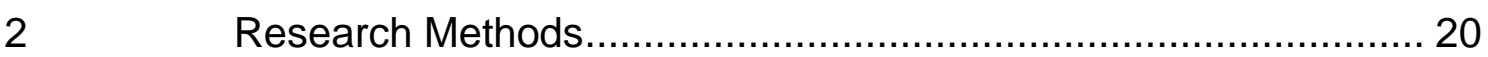

2.1 Design Science Research and Data Science Life Cycle............... 20

2.1.1 Modeling in Data Science projects............................................. 24

2.1.2 Main statistical methods used............................................... 25

2.2 Thesis projects and data science applications ............................ 32

3 Article 1 - Structure and Process Associated with the Efficiency of Intensive Care Units in Low-Resource Settings: An Analysis of the CHECKLIST-ICU Trial database ........................................................ 39

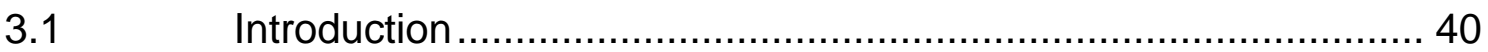

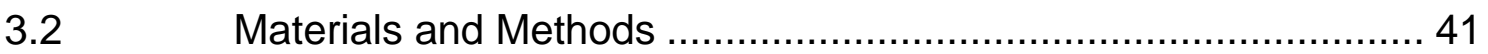

3.2.1 Study design and data source ……..................................... 41

3.2.2 Study population ................................................................. 41

3.2.3 Outcomes and ICU efficiency ................................................. 42

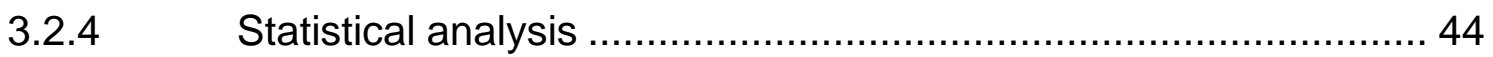

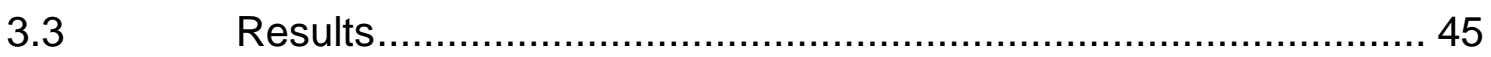

3.3.1 Efficiency groups and general characteristics ........................... 45

3.3.2 Adherence to processes of care and clinical results ................... 47

3.3.3 Association of structure and process with the efficiency groups

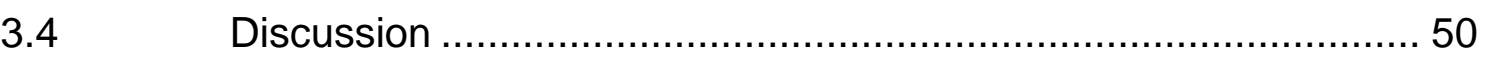

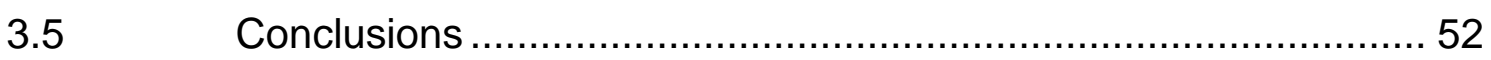

$4 \quad$ Article 2 - Comparing continuous versus categorical measures to assess and benchmark intensive care unit performance and efficiency....... 54

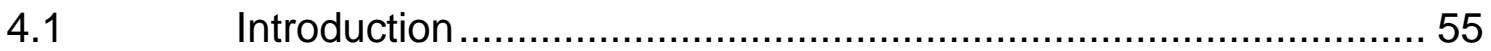

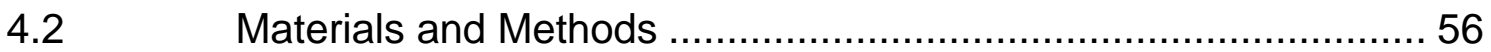

4.2.1 Study design and data source ………….............................. 56

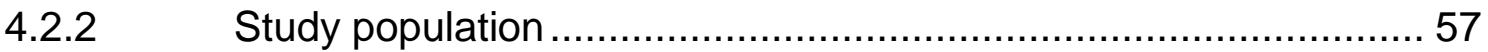


4.2.3 Outcomes and ICU performance ............................................ 57

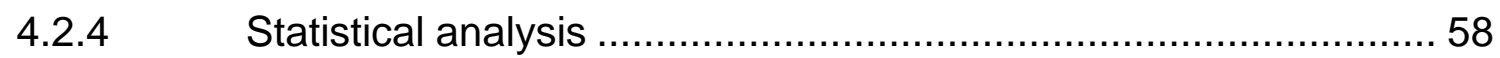

4.2.5 Advantages and disadvantages of SMR and SRU

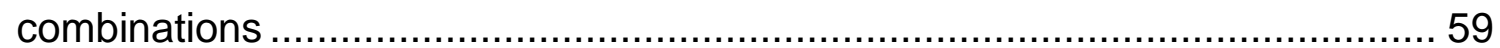

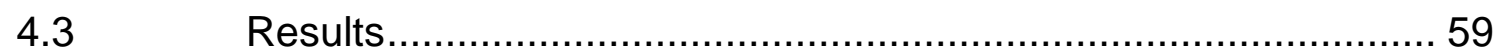

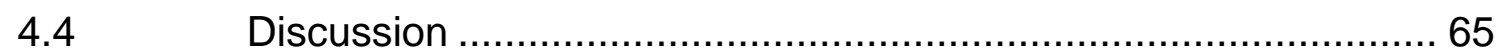

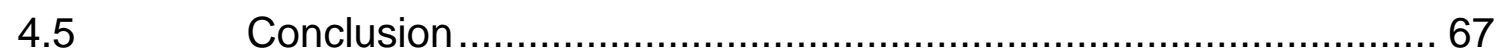

$5 \quad$ Article 3 - Increased number of nurses per bed is associated with higher efficiency in intensive care units: An analysis of the

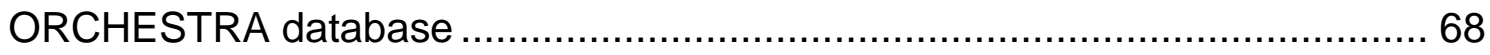

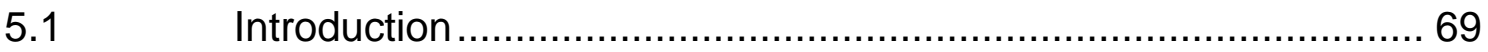

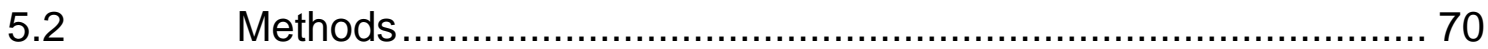

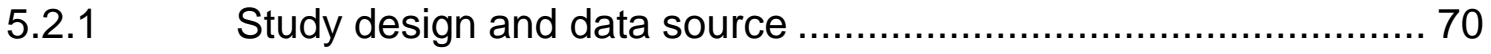

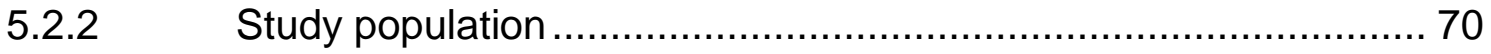

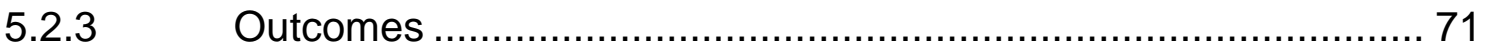

5.2.4 Statistical Analysis ............................................................... 71

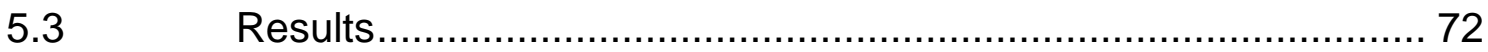

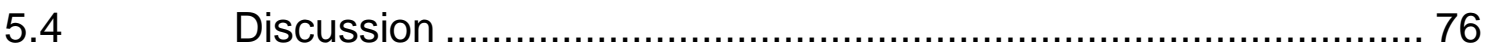

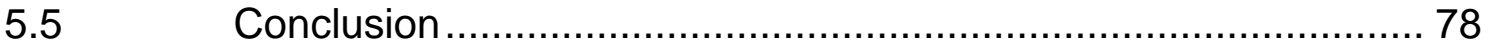

$6 \quad$ Article 4 - Characterisation of the first 250000 hospital admissions for COVID-19 in Brazil: a retrospective analysis of nationwide data

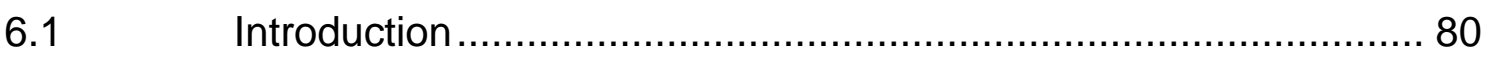

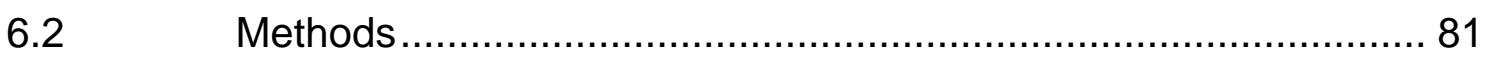

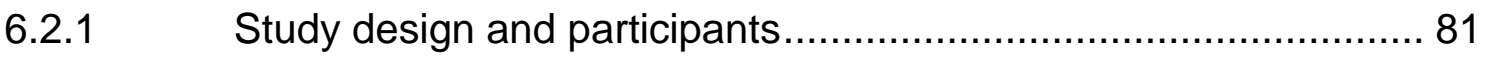

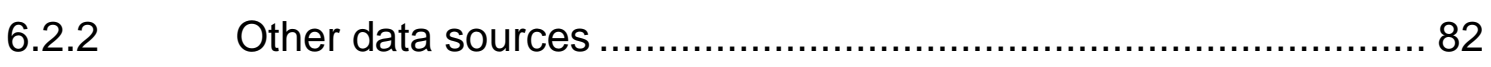

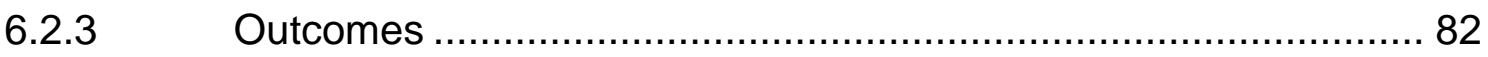

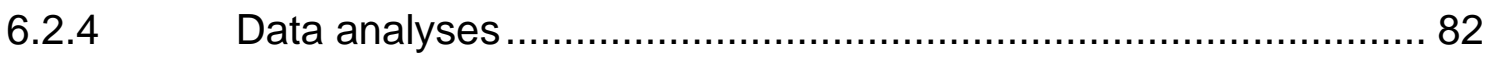

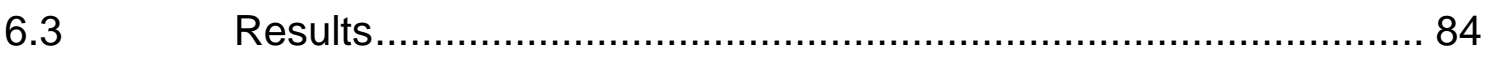

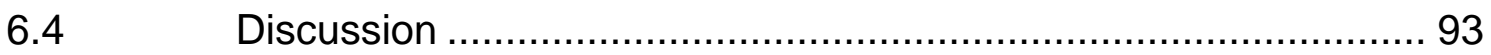

$7 \quad$ Article 5 - Severity, resource use and outcomes of COVID-19 hospital admissions in Brazil: comparison between the first and second wave

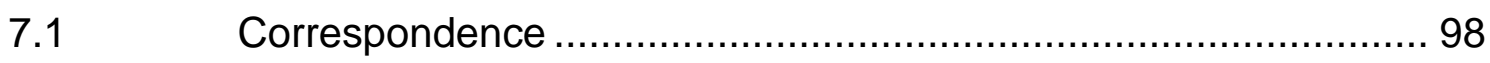

$8 \quad$ Article 6 - Evolving changes in mortality of 13,301 critically ill adult patients with COVID-19 over eight months......................................... 102

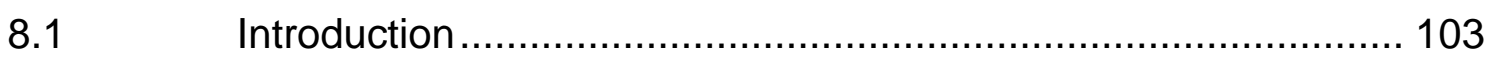




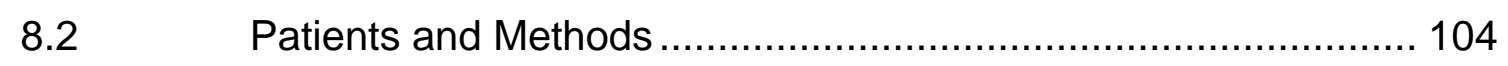

8.2.1 Study design and participants................................................. 104

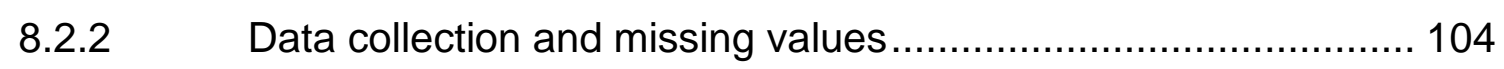

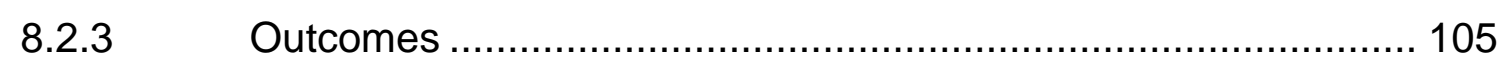

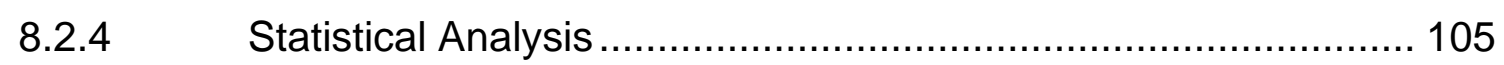

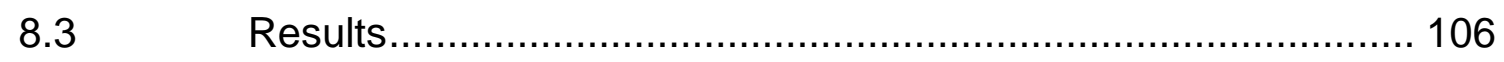

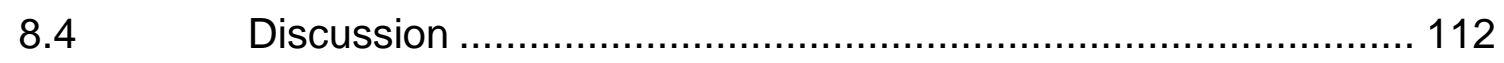

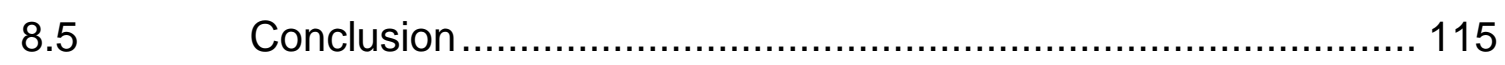

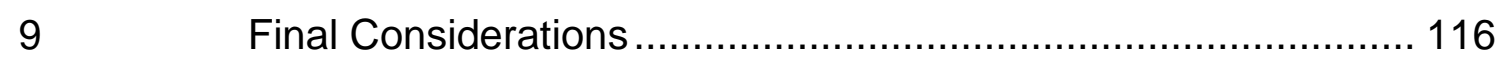

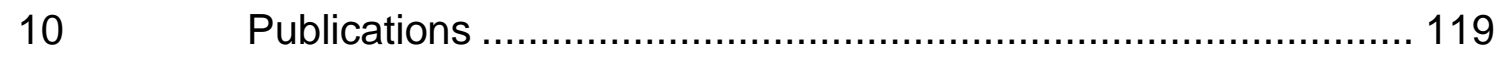

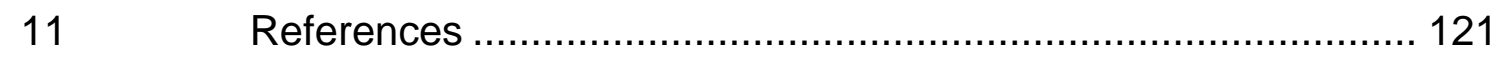

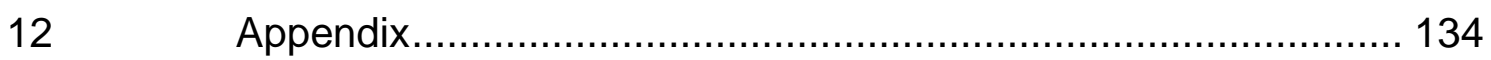

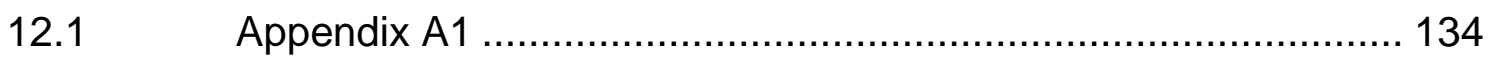

12.1.1 Appendix A1.1 - Calibration of SAPS-3 curves and the

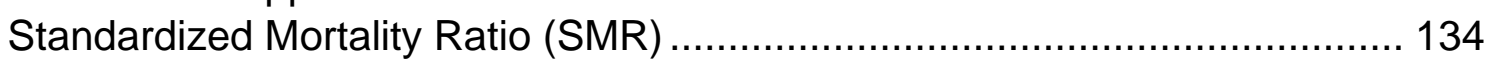

12.1.2 Appendix A1.2 - Standardized Resouce Use (SRU) calculation

12.1.3 Appendix A1.3 - List of Intensive care units ............................. 137

12.1.4 Appendix A1.4 - Organizational characteristics ......................... 138

12.1.5 Appendix A1.5 - Adaptive elastic-net hyperparameter estimation and confidence intervals ........................................................... 140

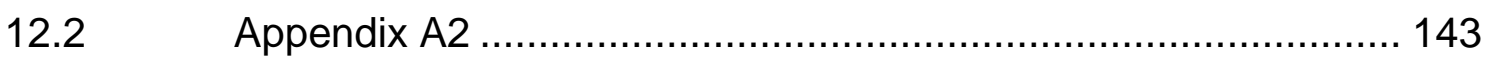

12.2.1 Appendix A2.1 - Calibration of SAPS-3 and APACHE-IV mortality risk models...................................................................... 143

12.2.2 Appendix A2.2 - Average length of stay for each SAPS-3 and

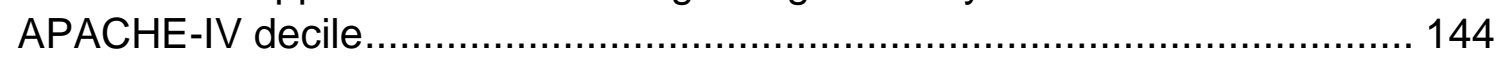

12.2.3 Appendix A2.3 - Distribution of performance metrics (SMR, SRU and ASER) per units stratified by efficiency groups ............................ 145

12.2.4 Appendix A2.4 - Distribution of SMR, SRU and ASER values

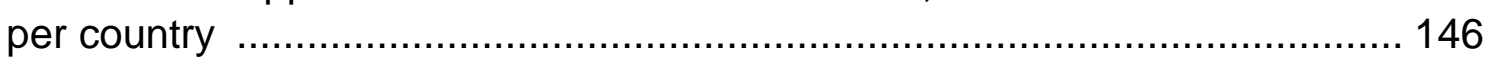

$12.3 \quad$ Appendix A4 ................................................................. 147

12.3.1 Appendix A4.1 - Data Coverage, data sources and variables.... 147

12.3.2 Appendix A4.2 - Multiple imputation procedure and results ....... 152

12.3.3 Appendix A4.3 - Supplementary analyses of COVID-19 hospital admissions, use of resources and outcomes ................................. 159

12.3.4 Appendix A4.4 - Sensitivity analyses .................................... 175

12.3.5 Appendix A4.5 - Comparison of multicenter COVID-19 cohort . 190 
12.4 Appendix A5

12.4.1 Appendix A5.1 - Data sources and study population

12.4.2 Appendix A5.2 - Average daily prevalence of Variants of Concern in Brazil

12.4.3 Appendix A5.3 - Comparison of hospital admissions and inhospital mortality between first and second COVID-19 waves in Brazil $(n=$ $1,187,840)$

12.4.4 Appendix A5.4 - Comparison of first and second waves of COVID-19 hospital admissions in Brazil. .

12.4.5 Appendix A5.5 - In-hospital mortality stratified by hypoxaemia,

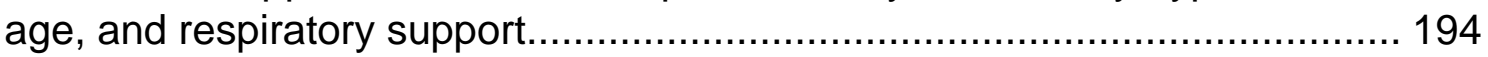

12.4.6 Appendix A5.6 - Changes in Mobility in Brazil .......................... 195

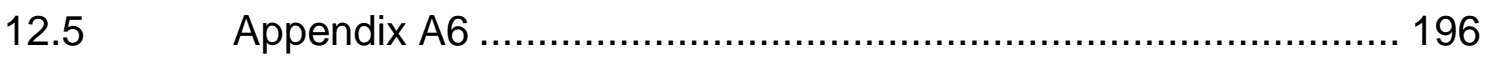

12.5.1 Appendix A6.1 - Supplemental Methods ................................ 196

12.5.2 Appendix A6.2 - Summary of hospitals and ICUs ..................... 198

12.5.3 Appendix A6.3 - Study Flowchart and ICU occupancy rates...... 199

12.5.4 Appendix A6.4 - Additional descriptive analyses 201

12.5.5 Appendix A6.5 - Modelling preparations, propensity scores and results 205

12.5.6 Appendix A6.6 - Sensitivity Analyses 


\section{List of tables}

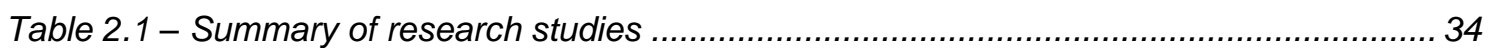

Table 3.1- General characteristics of all ICUs the efficiency groups. ...................................... 46

Table 3.2 - Efficiency groups and secondary outcomes of adherence to processes of care

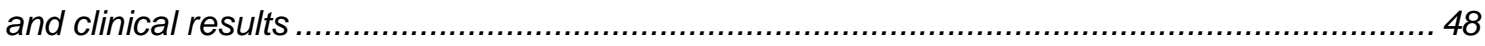

Table 3.3 - Cramer's $V$ values for the association between the characteristics of structure

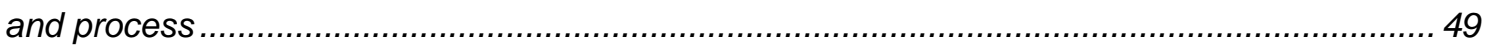

Table 3.4 - Results from the regularized multivariable logistic regression of the

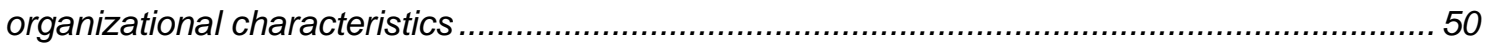

Table 4.1 - Characteristics and outcome metrics of Brazilian and Dutch ICUs ......................... 60

Table 4.2 - Characteristics and outcomes of critically ill patients in Brazil and The

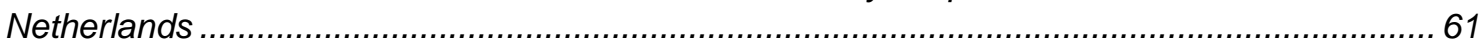

Table 4.3 - Advantages and disadvantages of using a categorical/dichotomous versus the

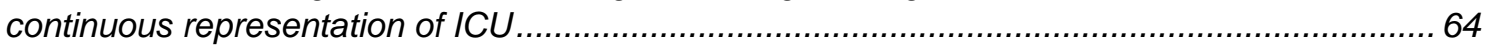

Table 5.1 - Characteristics and outcomes of Intensive Care Unit patients .............................. 73

Table 5.2 - Outcome measures and organizational characteristics of all Intensive Care

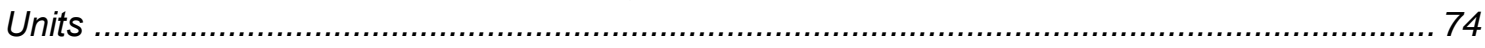

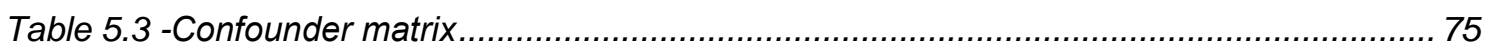

Table 5.4 -Estimates of effect for each organizational factor to the efficiency in the linear model with propensity scores and the causal random forests ................................................. 75

Table 6.1 - Patient characteristics stratified by region ......................................................... 86

Table 6.2 - Intensive care admission, need of respiratory support, ICU and in-hospital

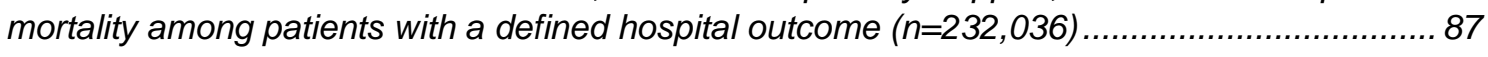

Table 6.3 - Demographic, administrative and health system regional characteristics ................ 90

Table 7.1 - Comparison of hospital admissions and in-hospital mortality between first and second COVID-19 waves in Brazil ( $n=1,187,840$ hospitalizations until May 01, 2021;

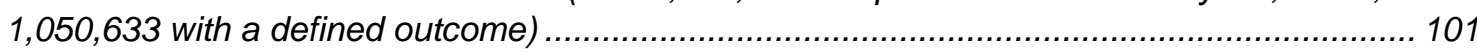
Table 8.1 - Clinical characteristics and outcomes of 13,301 critically ill COVID-19 patients,

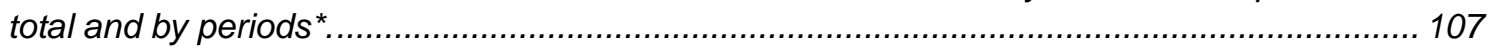
Table 8.2 - Characteristics and outcomes of critically ill patients stratified by advanced

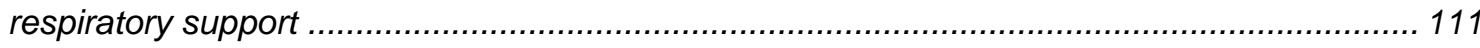

Table A12.1 - Comparison of average ICU length-of-stay per survivor patient and SAPS-3

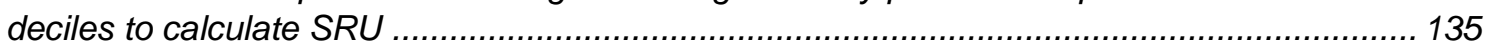

Table A12.2 - List of intensive care units and their performance indicators .......................... 137 Table A12.3 - Descriptive statistics and univariate analysis of structure and process

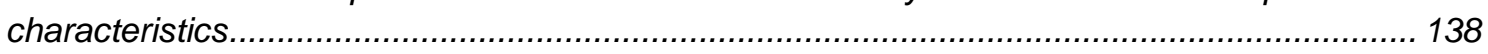

Table A12.4 - Intervals and final results of the AENet hyperparameter estimation .................. 141

Table A12.5 - Database coverage in terms of Brazilian municipalities and population ............ 147

Table A12.6 - Description of the data sources used in this study ........................................ 148

Table A 12.7 - Detailed description of variables used in the study ...................................... 149

Table A12.8 - Missingness pattern (proportion of missing values in assessed variables) on the population used in the main analysis (RT-qPCR confirmed) $(n=232,036$ with a defined

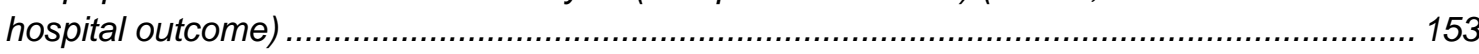

Table A12.9 - Missing vs. not missing comorbidities ( $n=232,036$, sample with defined

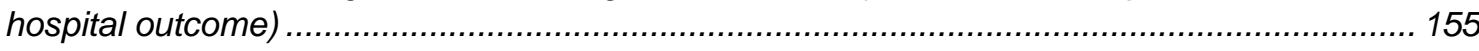

Table A12.10 - Missing ICU admission versus not missing ICU admission ( $n=232,036$ with a defined hospital outcome) 
Table A12.11 - Missing Respiratory support versus not missing Respiratory support (sample $n=232,036$ with a defined hospital outcome)

Table A12.12 - Comparison between complete-case and imputed values (sample $n=232,036$ with a defined hospital outcome).

Table A12.13 - Number of COVID-19 cases, hospitalisations, and in-hospital deaths, absolute and age and sex-adjusted rates per 100,000 population for each time frame of the pandemic and region of Brazil.....

Table A12.14 - Symptoms of hospitalised COVID-19 patients in Brazil and regions (sample $n=254,288)$

Table A12.15 -- Chronic comorbidities description of hospitalised COVID-19 patients in

Brazil (sample $n=254,288$ )

Table A12.16 - Times of the disease among patients with a defined hospital outcome (main analysis)

Table A12.17 - In-hospital mortality stratified by age and sex in Brazil

Table A12.18 - In-hospital mortality stratified by chronic comorbidities, level of education and self-reported race and age .....

Table A12.19 - In-hospital mortality stratified by ICU admission, respiratory support and age.

Table A12.20 - Health system burden in Brazil and its regions (number / per 100,000 inhabitants)

Table A12.21 - Overall in-hospital mortality, among those admitted to the ICU or under invasive mechanical ventilation, stratified by age and Brazilian regions

Table A12.22 - Hospitalisations and ICU admissions per hospital and ICU beds in Brazil and regions*

Table A12.23 - Use of resources in terms of proportions of hospitalised patients admitted

to the ICU and under invasive mechanical ventilation stratified by age and region.....

Table A12.24 - Patients characteristics stratified by region (Sensitivity Analysis: patients with laboratorial and clinical diagnosis of COVID-19)

Table A12.25 - Times of the disease, intensive care admissions and need of respiratory support among patients with a defined hospital outcome (Sensitivity Analysis: patients with laboratorial and clinical diagnosis of COVID-19)....

Table A12.26 - In-hospital mortality stratified by age and sex in Brazil (Sensitivity Analysis: patients with laboratorial and clinical diagnosis of COVID-19)

Table A12.27 - In-hospital mortality stratified by chronic comorbidities, level of education, self-reported race, and age (Sensitivity Analysis: patients with laboratorial and clinical diagnosis of COVID-19)

Table A12.28 - In-hospital mortality stratified by ICU admission, respiratory support and age (Sensitivity Analysis: patients with laboratorial and clinical diagnosis of COVID-19)

Table A12.29 - Health system burden in Brazil and its regions (number / per 100,000 inhabitants) (Sensitivity Analysis: patients with laboratorial and clinical diagnosis of COVID19)

Table A12.30 - Overall in-hospital mortality, among those admitted to the ICU or under invasive mechanical ventilation, stratified by age and Brazilian regions (Sensitivity Analysis: patients with laboratorial and clinical diagnosis of COVID-19)

Table A12.31 - Hospitalisations and ICU admissions per hospital and ICU beds in Brazil and regions (Sensitivity Analysis: patients with laboratorial and clinical diagnosis of COVID19)*.....

Table A12.32 - Use of resources in terms of proportions of hospitalised patients admitted to the ICU and under invasive mechanical ventilation stratified by age and region (Sensitivity Analysis: patients with laboratorial and clinical diagnosis of COVID-19)

Table A 12.33 - In-hospital mortality by comorbidities numbers, ICU admission and respiratory support in main analysis and multiple imputed data in Brazil (sensitivity analysis)

Table A 12.34 - In-hospital mortality by comorbidities numbers and age in main analysis and multiple imputed data in Brazil (sensitivity analysis) 
Table A 12.35 -In-hospital mortality by comorbidities numbers, ICU admission and respiratory support in main analysis and multiple imputed data stratified by age (sensitivity analysis)

Table A12.36 -List of Hospitals

Table A12.37 - Number of beds; ICU occupancy rates and proportion of patients in invasive mechanical ventilation before and after the pandemic....

Table A12.38 - Clinical characteristics and outcomes of 4,188 critically ill COVID-19 patients that required advanced respiratory support (noninvasive or invasive). Period 1 February 27th to April 25 th Period 2 - April $26^{\text {th }}$ to June $6^{\text {th }}$; Period 3 - June $7^{\text {th }}$ to August $10^{\text {th }}$; Period $4-$ August $11^{\text {th }}$ to October $28^{\text {th }}$

Table A12.39 - Distribution of patient's comorbidities in overall and stratified by the time periods defined by the estimated breakpoints (Period 1 - February $27^{\text {th }}$ to April $25^{\text {th }}$; Period 2 - April $26^{\text {th }}$ to June $6^{\text {th }}$; Period 3 - June $7^{\text {th }}$ to August $10^{\text {th }}$; Period 4 - August $11^{\text {th }}$ to October $\left.28^{\text {th }}\right)$

Table A12.40 - Clinical characteristics and outcomes of critically ill COVID-19 patients that underwent advanced respiratory support $(n=4,188)$, stratified by the first support used (IMV - invasive mechanical ventilation, NIRS - Noninvasive respiratory).....

Table A12.41 - Comparison of three multivariable logistic regression modelling approaches for propensity score estimation (patients that underwent advanced respiratory support, $n=$ 4,188)

Table A12.42 - Comparison full and reduced random-effects multivariable cox regression modelling approaches (patients that underwent advanced respiratory support, $n=4,188$ ) .....210 Table A12.43 - Random-effects multivariable cox regression model with SMR-weighting from propensity scores and adjusted by the time periods defined using the estimated breakpoints (Sensitivity analysis)

Table A12.44 - Random-effects multivariable cox regression model with inverse probability treatment weighting (IPTW) using trimmed propensity scores (percentile 95\%) and adjusted by the time periods defined using the estimated breakpoints (Sensitivity analysis)

Table A 12.45 -Comparison of three multivariable logistic regression modelling approaches for propensity score estimation in the subset of patients that presented $\mathrm{PaO}_{2} / \mathrm{FiO}_{2}$ values ( $n=1,963)$ and adjusted by the time periods defined using the estimated breakpoints (Sensitivity analysis).....

Table A12.46 -Random-effects multivariable cox regression model with inverse probability treatment weighting (IPTW) for the subset of patients that presented $\mathrm{PaO}_{2} / \mathrm{FiO}_{2}(\mathrm{~N}=1,963)$ values and adjusted by the time periods defined using the estimated breakpoints (Sensitivity analysis).....

Table A12.48 -Random-effects multivariable cox regression model with inverse probability weighting (IPTW) and multiple imputation by chained equations of $\mathrm{PaO}_{2} / \mathrm{FiO}_{2}$ values. The model was adjusted by the time periods defined using the estimated breakpoints 


\section{List of figures}

Figure 2.1 - Design Science research cycles (HEVNER, 2007; MANRESA PEREZ, 2020) ...... 21

Figure 2.2 -Data Science Life Cyle (MANRESA PEREZ, 2020; SHEARER, 2000)................... 22

Figure 3.1 - Efficiency matrix and the efficiency groups. ................................................. 45

Figure 4.1 Distribution of SMR, SRU, and ASER values in the efficiency matrix in (A)

Brazilian ICUs and (B) Dutch ICUs.... 60

Figure 4.2 - Association between SMR and SRU with ASER in (A) Brazilian ICUs and (B) Dutch ICUs. Bisector line represents a perfect correlation between metrics. The blue line is the regression line with confidence intervals (shaded area). $R^{2}$ was obtained from the regression line using the ASER as a predictor for SMR or SRU.

Figure 4.3 - Funnel plots for SMR, SRU and ASER in (A) Brazilian ICUs and (B) Dutch ICUs. Dashed lines represent control limits: dark blue - 95\%; and light blue-99.8\%. Theta is the baseline value for each metric obtained as the observed/expected value.

Figure 5.1 - Association between SMR and SRU with ASER in (A) Brazilian ICUs and (B) Dutch ICUs. Bisector line represents a perfect correlation between metrics. The blue line is the regression line with confidence intervals (shaded area). $R^{2}$ was obtained from the regression line using the ASER as a predictor for SMR or SRU. ........................................ 73

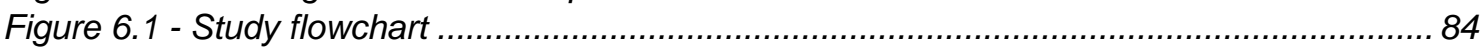

Figure 6.2 - Epidemic evolution shown in three-time frames in Brazil in terms of reported confirmed COVID-19 cases, hospitalisations and in-hospital deaths.

Figure 6.3 -In-hospital mortality stratified by age, sex, comorbidities, level of education, self-reported race*, intensive care admission and invasive mechanical ventilation for hospitalised COVID-19 patients in Brazil.

Figure 6.4 -Temporal evolution of COVID-19 hospitalisation rates per 100,000 adult population and crude in-hospital mortality in the five geopolitical regions of Brazil................... 91 Figure 6.5 -Health system burden and in-hospital mortality stratified by age in hospitalised COVID-19 patients in the five regions of Brazil.

Figure 7.1 - Temporal increase in the number of COVID-19 hospital admissions and deaths in Brazil stratified by severity (hypoxaemia), age and respiratory support ( $n=1,217,332$, all hospitalizations)....

Figure 8.1 - Progression of adult ICU admissions with COVID-19 from February 27th, 2020 to October 28th, 2020.

Figure 8.2 - Univariable survival curves (Kaplan-Meier) of factors related to the 60-day outcome in critically ill patients that underwent advanced respiratory support

Figure 8.3 - Random-effects multivariable cox proportional hazards model to assess the association of clinical characteristics and initial respiratory support with 60-day mortality in patients that underwent advanced ventilatory support (NIRS and/or IMV), adjusted by the time-period of admission. The hospital was considered as the random intercept (Standard deviation $=0.50$ ). To account for the nonrandomization, we used inverse-probability treatment weighting (IPTW) of propensity scores regarding the initial use of NIRS. We provide the Hazard Ratio (HR) for 60-day in-hospital mortality and its respective 95\% confidence intervals for each variable.

Figure A12.1 - Calibration belts for evaluating the calibration of SAPS-3 standard equation

Figure A12.2 - Missingness pattern for ICU, respiratory support, signs/symptoms and comorbidities 
Figure A12.3 - Epidemic evolution showed during three-time frames in Brazil with rates per 100,000 population

Figure A12.4 - In-hospital mortality stratified by age and sex accounting for the reported symptom of oxygen saturation < $95 \%$, number of comorbidities, ICU admission, and respiratory support.

Figure A12.5 - In-hospital mortality per age group for symptoms of Oxygen saturation < 95\%, Dyspnoea, respiratory distress, and SARI diagnosis.....

Figure A12.6 - Proportion of intensive care unit admission and use of invasive mechanical ventilation stratified by age in hospitalised COVID-19 patients in the five regions of Brazil..... 173 Figure A12.7 - In-hospital mortality stratified by age, sex, comorbidities, level of education, self-reported race *, intensive care admission and invasive mechanical ventilation for hospitalized COVID-19 patients in Brazil (Sensitivity Analysis: patients with laboratorial and clinical diagnosis of COVID-19)....

Figure A12.8 - Health system burden and in-hospital mortality stratified by age in hospitalised COVID-19 patients in the five regions of Brazil (Sensitivity Analysis: patients with laboratorial and clinical diagnosis of COVID-19)

Figure A12.9 - Proportion of intensive care unit admission and use of mechanical ventilation stratified by age in hospitalised COVID-19 patients in the five regions of Brazil (Sensitivity Analysis: patients with laboratorial and clinical diagnosis of COVID-19) ............................... 185

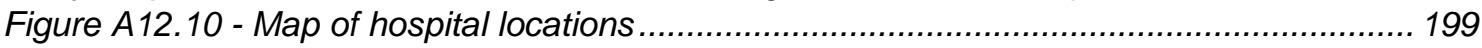

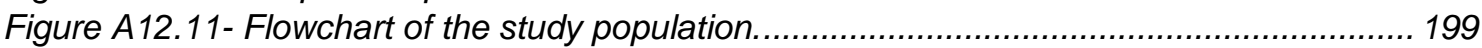

Figure A12.12 - Univariable survival curves (Kaplan-Meier) of factors related to the 60-day outcome in critically ill patients that underwent invasive mechanical ventilation or noninvasive respiratory support $(n=13,301)$.

Figure A12.13 - Univariable survival curves (Kaplan-Meier) 60-day outcome in critically ill patients that underwent advanced respiratory support $(n=4,188)$ stratified by Respiratory support considering patients that underwent oxygen support, noninvasive respiratory support (NIRS only), NIRS with subsequent intubation (NIRS failure) and invasive ventilation (IMV only). Differences among curves were assessed using the log-rank test with a confidence level of 0.05 .

Figure A12.14 - Distribution of 60-day in-hospital mortality per age, SOFA, and SAPS-3 from patients that underwent advanced respiratory support $(n=4,188)$

Figure A12.15 - Martingale residuals for Age, SOFA, and SAPS-3

Figure A12.16 - Distribution of propensity scores among those treated initially with noninvasive respiratory support (NIRS first) and those treated initially with invasive mechanical ventilation (IMV first)

Figure A12.17 - Proportion of COVID-19 patients that underwent steroids administration. .....217 


\section{List of Abbreviations}

AENET - Adaptive Elastic Net

AIC - Akaike information criteria

AMIB - Brazilian Association of Intensive Care (Associação Brasileira de Medicina Intensiva)

APACHE - Acute Physiology and Chronic Health Evaluation

ATE - Average Treatment Effect

AUROC - Area Under the Receiving Operating Characteristic curve

$B I C$ - Bayesian information criteria

Brazil CAAE - Committees and the Brazilian National Ethics Committee (Certificado de Apresentação de Apreciação Ética)

$\mathrm{Cl}$ - Confidence Interval;

CLABSI- Central-line associated bloodstream infection;

CNES - Cadastro Nacional de Estabelecimentos de Saúde

COPD - Chronic Obstructive Pulmonary Disease

COVID-19 - Coronavirus Disease 19

$\mathrm{CPH}$ - Cox proportional hazard regression model

CRF - Causal Random Forests,

CRISP-DM - Cross-Industry Standard Process for Data Mining

CVC-Central Venous Catheter;

DSLC - Data Science Life Cycle

$D S R$ - Data Science Research

EDA - Exploratory data analysis

Generalized linear model - GLM

GiViTI - Italian Group for the Evaluation of the Interventions in Intensive Care Units (Gruppo

Italiano per la Valutazione degli interventi in Terapia Intensiva)

HFNC - High-flow Nasal Cannula

$H R$ - Hazard Ratio

IBGE - Instituto Brasileiro de Geografia e Estatística

ICU - Intensive Care Unit

IHM - In-hospital Mortality

IMV - Invasive Mechanical Ventilation

IPTW - Inverse-probability Treatment Weighting

IQR- Interquartile Range;

KM - Kaplan-Meier

LASSO - Least Absolute Shrinkage and Selection Operator

LOS - Length-of-Stay

$L R$ - Logistic Regression

MAR - Missing at Random

MFI - Modified Frailty Index

MICE - Multiple Imputation by Chained Equations

$M L$ - Machine Learning

MLE - Maximum Likelihood Estimator

MV-Mechanical Ventilator;

NICE - National Intensive Care Evaluation

NIRS - Noninvasive Respiratory Support

NPPV - Noninvasive Positive Pressure Ventilation

OR - Odds Ratio

ORCHESTRA - Organisational CHaractEriSTics in cRitical cAre

RASS- Richmond Agitation-Sedation Scale;

RRT - Renal Replacement Therapy 
RT-PCR - Reverse transcription polymerase chain reaction

SAPS - Simplified Acute Physiology Score

$S A R I$ - Severe Acute Respiratory Infection

SARS - Severe Acute Respiratory Syndrome

SARS-CoV-2 - Severe Acute Respiratory Disease CoronaVirus 2

$S D$ - Standard Deviation

SES - State health departments (Secretaria Estadual da Saúde)

SIVEP-Gripe - Influenza Epidemiological Surveillance Information System (Sistema de Informação de Vigilância Epidemiológica da Gripe)

SMR - Standardized Mortality Ratio

SOFA - Sequential Organ Failure Assessment

$S R U$ - Standardized Resource Use

STROBE -

SUS - Brazilian Universal Health System (Sistema Único de Saúde),

UK - United Kingdom

UTI- Urinary tract infection;

VAP-Ventilator-Associated Pneumonia.

VoC - Variant of Concern

Vol - Variant of Interest

VTE - Venous Thromboembolism

WHO - World Health Organization 


\section{Introduction}

Healthcare systems aim to provide suitable treatment to patients. For this purpose, the decisions vary from state-of-the-art treatments to adequate use of resources. Management of resources is essential to satisfactorily treat patients and improve efficiency, especially under high strain conditions or limited resources, such as low-and-middle-income countries (BOZZA; SALLUH, 2010).

The use of resources is different among departments within a hospital or a system. One way to improve resource allocation is through benchmarking. In healthcare systems, benchmarking follows the Donabedian's framework and is divided into three perspectives (DONABEDIAN, 1988): structure, process and outcome. Several studies have identified targets for improvement in different departments with this framework to reduce costs and improve patient outcomes (AYANIAN; MARKEL, 2016).

One of the main departments in a healthcare system is the intensive care unit (ICU), which deals with complex cases, results in high costs, and provides relevant insights for treating severe diseases (GARLAND, 2005). In the intensive care field, benchmarking consists of quantifying units' performance using standardized measures to allow fair comparison (WOODHOUSE et al., 2009). Previous studies focused on benchmarking ICU's outcomes, since they are easy to collect, especially mortality (clinical efficacy) and use of resources (efficiency), measured by the Standardized Mortality Ratio (SMR) and the Standardized Resource Use (SRU), respectively (FLAATTEN, 2012; VERBURG et al., 2018).

Performing benchmarking in intensive care units is challenging (GARLAND, 2005). Factors such as the organizational culture, the availability of data, and the implementation of protocols may impact the adequate evaluation of the whole system. The challenge increases with an unexpected strain increase, mainly due to external or non-controlled factors such as a pandemic. Under these conditions, the healthcare system may provide unusual performance, with a sudden change of organizational procedures to deal with the high resource demand. 
On March 11, 2021, the World Health Organization (WHO) has declared the coronavirus disease (COVID-19) pandemic caused after the global outbreak of the severe acute respiratory syndrome coronavirus 2 (SARS-CoV-2) (WORLD HEALTH ORGANIZATION, 2020). The first confirmed case was in Wuhan, China, in December 2019 (ZHU et al., 2020), spreading to other Asian countries, Europe, and the Americas. Each healthcare system has dealt differently with the surge of cases trying their capacity with the increased demand for ICU resources, especially respiratory support.

This surge concerned specially low-and-middle-income countries (LMIC), where hospitals and ICU are limited (MURTHY; LELIGDOWICZ; ADHIKARI, 2015; RANZANI et al., 2021). One of those countries was Brazil, which became one epicenter of the COVID-19 pandemic. The first COVID-19 case was confirmed on February 26, 2020, and more than 17,7 million cases and 496,004 deaths were confirmed as of June $18^{\text {th }}, 2021$ (DONG; DU; GARDNER, 2020).

Brazil has one of the largest unified healthcare systems, providing universal care throughout the territory. It has an extensive national surveillance database that provided important data to understand the impact of the pandemic on healthcare resources (BASTOS et al., 2020a; RANZANI et al., 2021). Previous studies have shown that the progression of the COVID-19 pandemic started in State capitals and moved to inner regions, being locations with more and fewer healthcare resources in the country (CANDIDO et al., 2020; RANZANI et al., 2021). The pandemic has also intensified social-economic and regional inequalities, which may have influenced healthcare access and outcomes (BAQUI et al., 2020; DANTAS et al., 2021; PERES et al., 2021).

Understanding the best and worst-performing units using benchmarking provides essential insights for better allocation of resources. In addition, evaluating the course of the pandemic and its impact on resource use and outcomes assist decision-makers in better actions for pandemic mitigation and control and improve patient outcomes. Hence, this thesis consists of a collection of studies addressing these two major topics. 


\section{1}

\section{Research topics and objectives}

Two major research topics guide this thesis: the benchmarking or analysis of performance before the COVID-19 pandemic and evaluating outcomes and use of resources during the pandemic. In the first, the main research question was "What does drive the performance of healthcare systems?"; in the second part, the question was "What is the impact of COVID-19 pandemic in the use of resources and outcomes of healthcare systems?". Hence, this thesis consists of a collection of research studies on evaluating healthcare systems, use of resources, and outcomes, both pre and during the COVID-19 pandemic.

These research questions motivated six research studies in this thesis, from which four were already published, and two are still ongoing. A summary of those research studies is presented in Section 2.1.

\section{2}

Thesis structure

This thesis is structured as follows: this first chapter corresponds to the Introduction, providing background on the research topics, research questions, and objectives. Chapters 2 comprises the research methodology, analytical methods, and main research steps that guided this thesis. Chapters 3 to 8 are composed each by the research studies that follow this thesis. Chapter 9 provides the final considerations of the research studies and suggestions for future work. Chapter 10 summarizes all publications that occurred during this thesis's research period, with bibliographic reference and journal information. Chapter 11 is a single list of references cited in this thesis. Chapter 12 corresponds to the single appendix section comprised of the supplementary material of the six articles. 


\section{2 \\ Research Methods}

This section described the Data Science Life Cycle, a general framework for executing data science projects and extracting important insights from databases. We addressed each proposed research question as individual Data Science projects. In addition, we described the main methods used for data analysis in each project inside the life cycle.

\section{1}

\section{Design Science Research and Data Science Life Cycle}

This thesis followed a scientific methodology based on Design Science Research (DSR). The DSR is a paradigm that guides research focused on finding solutions or designs artefacts to solve daily problems and improve the activities of professionals (DRESCH; LACERDA; ANTUNES JR, 2015). Different from research in Social or Natural sciences, the DSR promotes the creation of artefacts (e.g., products, tools, information) to produce scientific knowledge in several areas (DRESCH; LACERDA; ANTUNES JR, 2015; MANRESA PEREZ, 2020). In this thesis, our research questions provided the initial motivation and problems to solve. Then, we combined the healthcare and epidemiology field knowledge with insights from statistical models' data and provided insights and artefacts to assist physicians, healthcare professionals, and decision-makers.

In brief, the DSR is composed of three main cycles (HEVNER, 2007) as illustrated in Figure 2.1: Design - in this cycle, the artefacts are designed, developed, evaluated, and improved to address the problem to be solved; Relevance - connects the main opportunities and demands to develop the artefact with the professionals and context of application; and the Rigor - consists in the knowledge foundation to guide the artefact development and guarantee the innovation and research relevance (e.g., scientific research, theories, frameworks). 


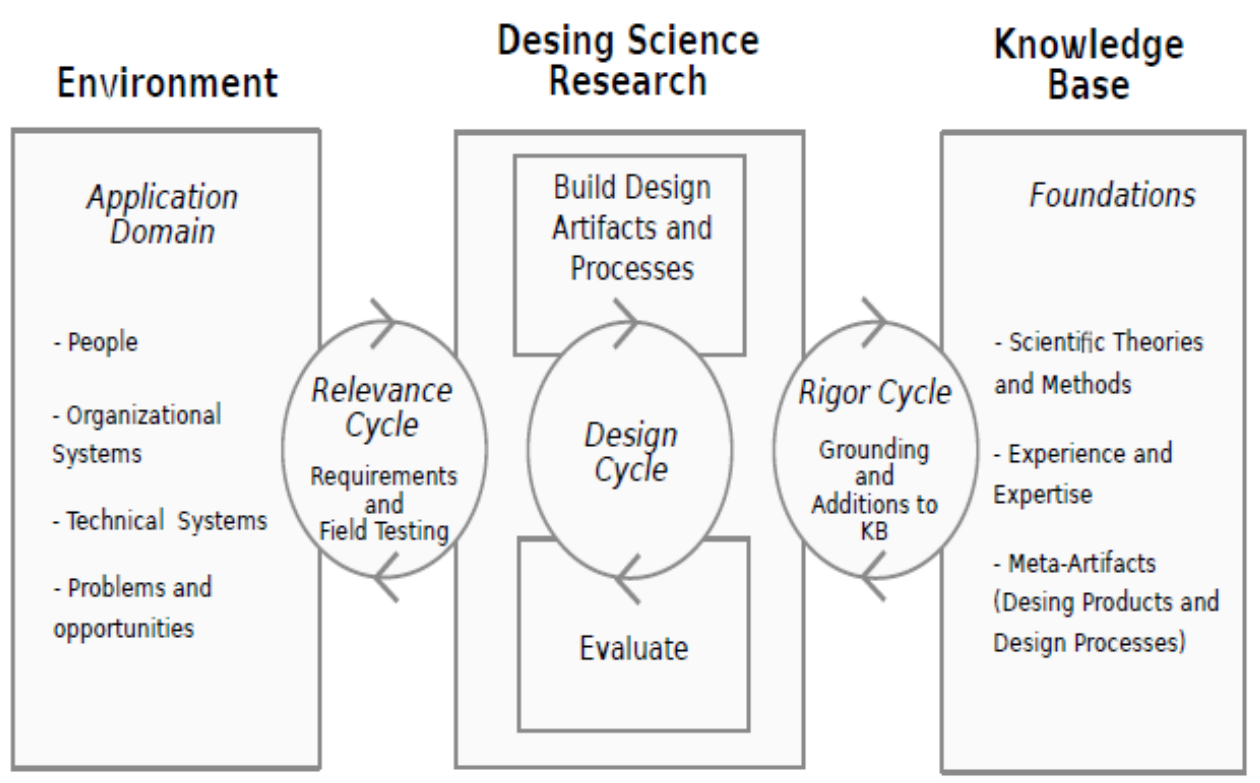

Figure 2.1 - Design Science research cycles (HEVNER, 2007; MANRESA PEREZ, 2020)

In the DSR paradigm, a work method is required to guide the research questions and develop the artefact (MANRESA PEREZ, 2020; NISBET; ELDER; MINER, 2009). In this thesis, we considered extracting knowledge from data to improve the comprehension of the context and problems and assist decision-making to the resource management in healthcare. Hence, we addressed each research question as a Data Science project, following the Data Science Life Cycle (DSLC).

We used the Cross-Industry Standard Process for Data Mining (CRISP-DM) among the several frameworks to explore and obtain information from data. The CRISP-DM is a framework that provides steps to guide a data mining project within the DSLC (SHEARER, 2000): Business Understanding, Data Understanding, Data Preparation, Modelling, Evaluation, and Deployment. We note that the steps are progressive but iterative and cyclical, allowing the incorporation of new questions or hypotheses, new experiences from business knowledge, and improving the final artefact. Each step or phase is briefly described as follows (Figure 2.2). 


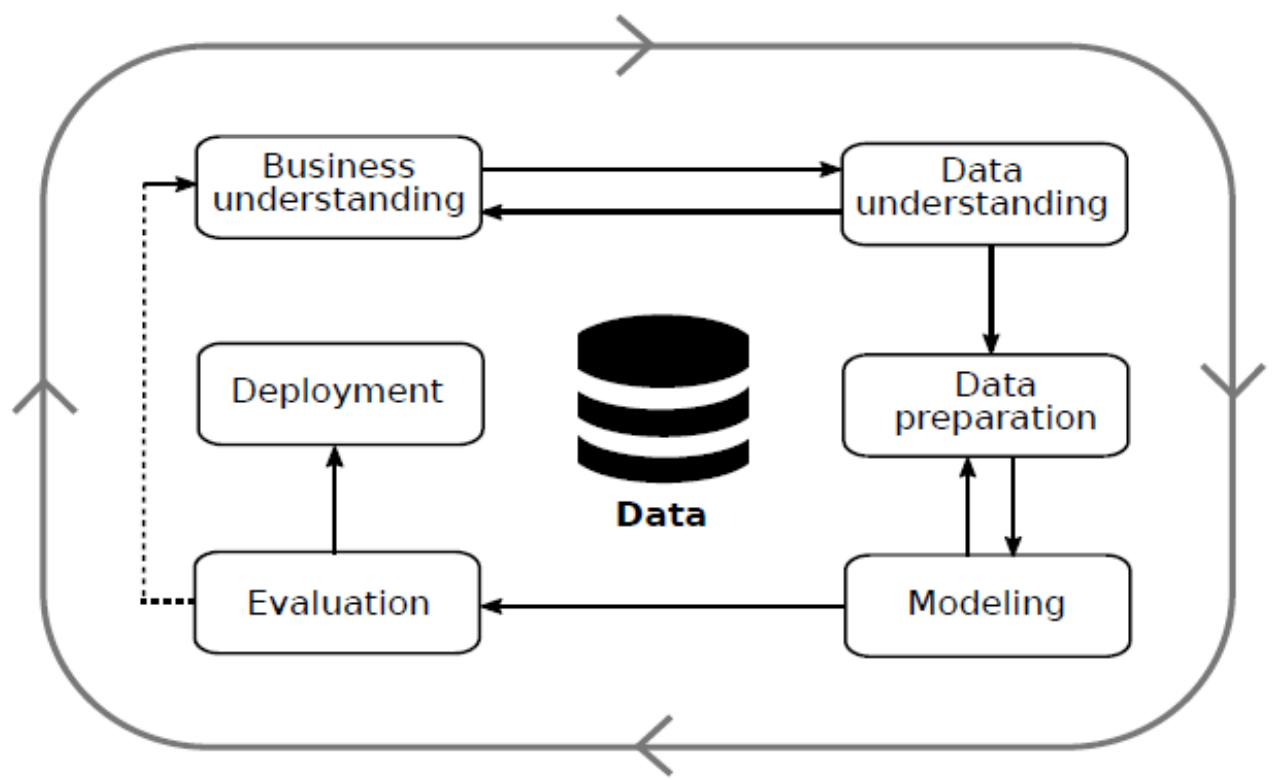

Figure 2.2 -Data Science Life Cycle (MANRESA PEREZ, 2020; SHEARER, 2000)

a) Business Understanding: This phase focuses on understanding the main research questions and business needs to be addressed. Therefore, the researcher defines the questions, the potential methods for addressing them, and the expected goals to solve them. Information from the environment, such as stakeholders and (business) specialists, also plays an important role in providing a good definition of the questions. In this thesis, our research questions were based on the previous knowledge of their corresponding healthcare field of study with inputs from healthcare specialists to refine the objectives and comprehend the needs to address them.

b) Data Understanding: This phase mainly comprises collecting, describing, and assessing the available data. Exploratory data analysis (EDA) comes into play to provide a deep understanding of what and when are the available data sources, potential biases, and data quality problems (e.g., missing values, inconsistencies), and what are the main characteristics and variables and their distributions. Descriptive statistical methods are used to obtain the main statistics from each variable and identify potential data errors and spurious values that could be addressed. Imputation may also be considered for missing values. In this thesis, our data sources were mainly healthcare databases of national surveillance and monitor systems used for decision-making and benchmarking. We evaluated data quality 
and refinements with the assistance of healthcare specialists to improve further analysis.

c) Data Preparation: One prepares the (final) dataset to extract information in this phase. This process may comprehend the selection of variables of interest to address the research questions, data cleaning, filtering, feature engineering, and format. In this thesis, we defined the study population - the final data sample and the variables or characteristics to be analyzed in the study.

d) Modelling: This phase comprehends the application of models and algorithms to identify patterns and extract relevant information from data. Depending on the research or business goal, a different model or several models may be considered, from an extensive list of data-driven models based on, for instance, machine learning or statistical models. Besides the model application, one should also evaluate its fitness to the research question and assess its results, using metrics for comparison or statistical tests. In this thesis, each research question considered a set of methods to evaluate the relationship among characteristics with a single response or outcome variable, mainly using statistical models. We briefly describe the main statistical methods considered in this thesis in Section 2.1.1.

e) Evaluation: This thesis evaluates if the model or data analysis method results were satisfactory to address the research question. Additional questions or hypotheses may arise to refine the previous steps and provide better results. Hence, the researcher may plan the next steps for the study and how to address them further. We described the main findings, discussed the potential drivers for those results and implications from the healthcare field, and the main limitations that can be overcome in future studies.

f) Deployment: In this "final" phase, one releases or deploys the main findings and artefacts obtained from the project to the environment. Feedback from users or other parties can be incorporated into the cycle to improve the results. In this thesis, each research question motivated research studies from which the results and knowledge built were reported as research articles. A few of those questions promoted the development of models and dashboards (tools) publicly available for the community. 


\subsection{1 \\ Modeling in Data Science projects}

The objective of the modeling phase is to extract patterns and obtain relevant information from data to respond to the research question. Technically, one aims to estimate a functional form to obtain a signal from data (expected pattern) and separate it from the random variation (noise). This process has two major objectives: named "inference" and "prediction", respectively (HARRELL, 2015).

When making Inference, one aims to identify or establish a relationship, correlation (also referred to as "association") or causal, between one or more variables (JAMES et al., 2021). In these settings, the research question provides one or more hypotheses to be verified and potentially confirmed. The researcher creates the (statistical) analysis plan by defining the variables of interest and the expected relation to be tested. For instance, one may be interested in identifying which organizational aspects provide better performance in hospitals or which characteristics from a patient are associated with increased odds of survival.

Regarding methods, statistical models play a significant role in Inference studies since they estimate the uncertainty around the hypothesis. The main methods or models are regression analysis and hypothesis testing. Assumptions are made to evaluate the hypothesis regarding a specific single estimator or a combination of variables.

On the other hand, Prediction consists of extracting patterns and relationships from a set of variables within a dataset to repeat them to newly available data (JAMES et al., 2021). Prediction models correspond to estimate a function in which a target variable (response) is explained by a set of inputs (predictors). In this context, the goal is to perform the best prediction in newly available data (generalization), as this model can offer inputs for future decisions. One example is prognostic modeling, in which a patient's conditions are inputs to estimate the risk of death for certain diseases.

One of the most often used prediction models is regression modeling. The response variable is a function of predictors and the noise or natural variability (HASTIE; TIBSHIRANI; FRIEDMAN, 2009). When the mapping function between response and predictors is linear, it is called "linear regression". However, transformations of the response variable or different mapingp functions may be 
necessary, extending those models to different contexts and improve the model fitness. This class of models is called the "generalized linear model" (GLM), and typical examples are linear regression and logistic regression, in which the response variable is numeric or binary (two classes), respectively.

In addition to regression modeling, machine learning (ML) has been developed to improve state of the art in prediction. ML provided more flexibility to the estimated function, allowing non-parametric modeling, and high dimensional settings (number of predictors greater than the number of observations), which extended some of the assumptions from previous models (JAMES et al., 2021). ML models presented better results than classic regression methods in certain settings, especially when different models are combined to make predictions, which is called ensemble (HASTIE; TIBSHIRANI; FRIEDMAN, 2009; JAMES et al., 2021). Examples of widely used machine learning models are the tree-based models such as Random Forest and Artificial Neural Networks (HASTIE; TIBSHIRANI; FRIEDMAN, 2009; JAMES et al., 2021).

Statistical models and machine learning algorithms can be applied in both Inference or Prediction perspectives. For instance, regression models have used regularization methods to address high dimensional variables and improve model fitting and prediction performance (JAMES et al., 2021). Also, tree-based models have been reformulated to obtain good statistical estimates for identifying causal relationships, such as the Causal Random Forests (ATHEY; TIBSHIRANI; WAGER, 2019).

\subsection{2}

\section{Main statistical methods used}

In this thesis, we used different methods to perform Inference over healthcare data. We focused on applying statistical methods to describe the study sample and model the relationships and hypothesis using clinically relevant variables under each data science project. We then evaluated models' goodness-of-fit, and their results were validated regarding the hypothesis tested and the adequacy to the research question. We briefly describe the main methods and techniques used for data analysis in each project:

a) Descriptive Statistics 
Descriptive Statistics consists of describing data distributions in the dataset. Descriptive methods are widely used for an initial analysis and assessment of data quality and potential correlations. For this purpose, we evaluate statistical estimates for central tendency - e.g., mean, median or mode, dispersion measures - e.g., standard deviation, range, or interquartile range (AMBROSIUS, 2007). In addition to those statistics, visual data distribution is assessed using histograms, boxplots, bar plots, or scatterplots.

b) Regression analysis

The linear relationship between two variables is often assessed using correlation coefficients, such as Pearson's correlation coefficient or Spearman's rho. Further analysis is conducted using regression models (HARRELL, 2015): where a specific random variable of interest, called "dependent" or "response" variable, is a function of another single or many random variables, called "independent" or "predictor" variables. The most common and simple model is Linear Regression.

Linear Regression assumes that the expected value of the dependent variable is a linear combination of the independent variables and a random error due to natural variability (Equation 1).

$$
E(\boldsymbol{Y} \mid \boldsymbol{X})=\boldsymbol{X} \widehat{\boldsymbol{\beta}}+\boldsymbol{\varepsilon}
$$

Where:

$\boldsymbol{Y}$ is the vector of the response variable

$\boldsymbol{X}$ is the matrix of predictors` values

$\widehat{\boldsymbol{\beta}}$ is the vector of the estimated coefficients

$\boldsymbol{X} \widehat{\boldsymbol{\beta}}$ is the linear combination of predictors, $\boldsymbol{X} \widehat{\boldsymbol{\beta}}=\widehat{\beta_{0}}+\widehat{\beta_{1}} X_{1}+\widehat{\beta_{2}} X_{2}+\cdots+$ $\widehat{\beta_{p}} X_{p}$, and $p$ is the number of predictors $\left(X_{0}=1\right)$

$\boldsymbol{\varepsilon}$ is the error or residual, where $\boldsymbol{\varepsilon} \sim N\left(0, \sigma^{2}\right)$

The $\hat{\beta}$ coefficients are estimated using the Least Squares estimator (JAMES et al., 2021). Those coefficients are helpful to understand the independent variable's contribution either as the rate of change (slope) of a line or plane or the effect size 
of this variable in explaining the response depending on the analysis or business objectives. For instance, one could evaluate the relationship between height and weight and understand how much weight increases with height and predict weight using height values.

The linear model is advantageous in several statistical analyses, especially when performing Inference. However, in some situations, the response variable may present different assumptions of the residual component, which requires the linear model to be extended. This more general set of models are called generalized linear models (GLMs), in linear models include transformations of the response or predictor variables using link functions.

In this thesis, we widely used Logistic Regression (LR), a GLM in which the response variable is a categorical variable of two classes (HARRELL, 2015; WALKER; DUNCAN, 1967). Most commonly, the Y is modelled as a binary or indicator variable $\{0,1\}$, in which " 1 " corresponds to the class of interest (or "positive class") and zero is the counterpart or baseline reference (or "negative class"). The modelling of the response variable is provided using a binomial link function. Hence, the LR model provides probabilities and measures of associations with respect to the class of interest. Examples in which response variables are binary classes are the patient's outcome (discharge/death) or the results of a diagnostic test (positive/negative). The mathematical formulation of the LR model is:

$$
P(\boldsymbol{Y}=1 \mid \boldsymbol{X})=\frac{1}{\left(1+e^{\boldsymbol{X} \boldsymbol{\beta}}\right)}
$$

Where:

$P(\boldsymbol{Y}=1 \mid \boldsymbol{X})$ is the conditional probability of $Y$ (the binary response variable) to be equal 1 , given the set of predictors $X$

$\boldsymbol{X}$ is matrix of predictor`s values

$\boldsymbol{\beta}$ is the vector of the estimated coefficients

In the LR, the linear assumption is made to the logit of the response variable $(\operatorname{logit}(P)$, Equation 3), a mathematical transformation that allows the model to become linear in the logarithm. Hence, the estimation of $\beta$ coefficients is possible 
in linear settings using the Maximum Likelihood Estimator (MLE) (HARRELL, 2015; JAMES et al., 2021).

$$
\operatorname{logit}(P)=\log \left(\frac{P}{1-P}\right)
$$

Where:

$P=P(Y=1 \mid X)$, and $\frac{P}{1-P}$ is the odds.

Similar to the linear regression model, the LR's coefficients represent the contribution of the predictors to the response. Since the response variable was transformed, the coefficients now represent the change in $\log$ odds, i.e., the rate of change in the logit of the $\mathrm{Y}$ for each unit of $\mathrm{X}$. For some studies, the log odds do not provide a direct interpretation. Hence, one prefers to obtain the odds ratio $(O R)$. The odds ratio is a measure of association between two variables, and in the LR model, it corresponds to the exponential of the log odds (BLAND, 2000). An $O R=$ 1.00 corresponds to a $\log$ odds $=0$, indicating that $X$ does not influences in $Y$. Therefore, if the $O R>1.00$, the odds for $Y$ are increased in $X$; and if the $O R<$ 1.00 the odds are decreased.

c) Regularization

The assumptions of regression models and GLMs are maintained under settings where the sample size $(n)$ is large. However, when the number of independent variables $(p)$ increases, the model fitness may reduce. Two major problems arise when $\mathrm{p}$ approaches $\mathrm{n}$ : overfitting and curse of dimensionality. Overfitting occurs when data is precisely modelled by the statistical model, often when the number of predictors is larger than necessary. In this case, the model fits precisely the data used for its estimation, but its performance and fitness are reduced when applied to new data. When the number of predictors approaches $(p=n)$ or becomes larger than the sample size $(p \gg n)$, also known as "high-dimensionality settings"), data become sparse, which increases the need for a larger sample and harms some properties of the LSE or MLE. Subsequently, the estimated coefficients may not be statistically reliable. With a large number of predictors, the problem of collinearity increases. 
To overcome these limitations, regularization methods have been applied to classic regression models or GLMs (JAMES et al., 2021). Regularization consists of adding a penalization parameter to the conventional regression coefficients. This method improves prediction accuracy. In addition, it allows an increased number of predictors, even larger than the sample size, and manages multicollinearity (BARRETT; LOCKHART, 2019; HASTIE; TIBSHIRANI; FRIEDMAN, 2009).

We consider two main types of penalties: the L1 and L2-norm. The first comprehends penalties in the absolute values of the coefficients, whereas the second is the penalty on the squared values (BARRETT; LOCKHART, 2019; HASTIE; TIBSHIRANI; FRIEDMAN, 2009). Each penalty also provides different properties. L1 and L2-norm also define the LASSO (Least Absolute Shrinkage and Selection Operator) and Ridge regression models, respectively.

The L1-norm allows coefficients to be reduced to zero, which features a variable selection approach. The mathematical formulation of LASSO regression is displayed in Equation 4:

$$
\widehat{\boldsymbol{\beta}}(L A S S O)=\left\{\operatorname{argmin}_{\boldsymbol{\beta}}\|\boldsymbol{y}-\boldsymbol{X} \boldsymbol{\beta}\|_{2}^{2}+\lambda\|\beta\|\right\}
$$

Where:

$\widehat{\boldsymbol{\beta}}$ (LASSO): the vector of the estimated coefficients

$\lambda$ penalty factors for the $\mathrm{L} 1$-norm

$\boldsymbol{y}$ : vector of the response values $(n \times 1)$

$X$ : matrix of the predictor values $(n \times p)$

$\|\beta\|:$ is the L1-norm term, where $\|\beta\|=\sum_{j=1}^{p}\left|\beta_{j}\right|$, and $p$ is the number of variables

The L2-norm has an identical structure. Due its squared power in the L-norm, it does not shrink the predictor' coefficient to zero, thus enabling, all variables to present a coefficient value through shrunken, including correlated predictors. The mathematical formulation for the Ridge Regression's estimator is displayed in Equation 5

$$
\widehat{\boldsymbol{\beta}}(\operatorname{Ridge})=\left\{\operatorname{argmin}_{\beta}\|\boldsymbol{y}-\boldsymbol{X} \boldsymbol{\beta}\|_{2}^{2}+\lambda\|\beta\|^{2}\right\}
$$

Where:

$\widehat{\boldsymbol{\beta}}($ Ridge $)$ : the vector of the estimated coefficients

$\lambda$ penalty factors for the $\mathrm{L} 2$-norm 
$\boldsymbol{y}$ : vector of the response values $(n \times 1)$

$X$ : matrix of the predictor values $(n \times p)$

$\|\beta\|^{2}$ : is the L2-norm term, where $\|\beta\|^{2}=\sum_{j=1}^{p} \beta_{j}{ }^{2}$, and $p$ is the number of variables

Compared to nonregularized regression models, regularization has shown improvement in model prediction. However, when making Inference, those penalized coefficients does not provide statistical inference properties (ZOU; ZHANG, 2009). To overcome this limitation, bias-adjustment methods have been applied, such as the adaptive LASSO and Adaptive ElasticNet. Those models provide unbiased estimators with asymptotic distribution (oracle properties), thus allowing the estimation of confidence intervals and accounting for the uncertainty in the estimated effect size.

In this thesis, we explored the Adaptive ElasticNet (AENET). The AENET is a regularization method based on the elastic-net regularization in which L1 and L2 norm penalties are added to estimates/coefficients to reduce their variance with the application of bias. However, to diminish the bias, AENET considers adaptive weights to coefficients and, therefore, can provide the oracle properties of its estimator (ZOU; ZHANG, 2009). Therefore, we chose to perform a multivariable analysis using the AENET model.

The AENet estimator is:

$$
\widehat{\boldsymbol{\beta}}(\text { AENet })=\left(1+\frac{\lambda_{2}}{n}\right)\left\{\operatorname{argmin}_{\beta}\|\boldsymbol{y}-\boldsymbol{X} \boldsymbol{\beta}\|_{2}^{2}+\lambda_{2}\|\beta\|_{2}^{2}+\lambda_{1} \sum_{j=1}^{p} \widehat{w}_{j}\left|\beta_{j}\right|\right\}
$$

Where:

$\widehat{\boldsymbol{\beta}}(A E N e t)$ : the vector of the estimated coefficients

$\lambda_{1}$ and $\lambda_{2}$ : penalty factors for the L1 and L2 norms, respectively

$\widehat{w}_{j}$ : adaptive weights, defined as $\widehat{w}_{j}=(|\hat{\beta}(E N e t)|)^{-\gamma}$, which consists of the scaled coefficients from an elastic-net or ridge regularization initially fitted to the data, where $\gamma$ is the scaling factor

$\boldsymbol{y}$ : vector of the response values $(n \times 1)$

$X$ : matrix of the predictor values $(n \times p)$

$\left|\beta_{j}\right|$ : is the L1-norm term 
$\|\beta\|_{2}^{2}$ : is the L2-norm term

d) Survival Analysis

Survival analysis comprehends a set of methods and models to evaluate the occurrence of events in time (KLEINBAUM; KLEIN, 2012). This concept arises from the analysis of the time for a failure to occur. In biostatistics, "survival" corresponds to the risk of death in a defined period, e.g., death or survival in 60days. However, extended modelling examples consist of time to intubation or time to a defined outcome.

Survival curves such as from the Kaplan-Meier estimators are one of the main methods to assess a patient's outcome progression in time $t$ (KLEINBAUM; KLEIN, 2012). Regression models can also be applied to evaluate the relationships of a variable with the response; however, in this case, the response is a time-toevent variable. The most common is the Cox proportional hazard regression model $(\mathrm{CPH})$.

The $\mathrm{CPH}$ models the time-to-event response as a function of predictors (COX, 1972; HARRELL, 2015; KLEINBAUM; KLEIN, 2012). The CPH model is composed of the baseline hazard function and the linear predictor. The hazard function () consists of the event's risk at the time, which is updated by the linear predictor, a linear combination of independent variables or predictors. The mathematical formulation for the $\mathrm{CPH}$ model is displayed in Equation 7 (COX, 1972; HARRELL, 2015; KLEINBAUM; KLEIN, 2012).

$$
\boldsymbol{h}(\boldsymbol{t}, \boldsymbol{X})=h_{0}(t) e^{\boldsymbol{X} \boldsymbol{\beta}}
$$

Where:

$h(t, X)$ : indicates that the hazard of the event to occur at time $t$ and given the predictors $X$

$h_{0}(t)$ is the baseline hazard, which is there reference risk of the event occur at time $t$ independent of the predictors

$\boldsymbol{X} \boldsymbol{\beta}$ is the linear combination of predictors values and coefficients independent of time $t$. It is also called the "linear predictor". $\boldsymbol{X} \boldsymbol{\beta}=\sum_{j=1}^{p} \beta_{j} X_{j}$

$\boldsymbol{\beta}$ is the vector the predictor's coefficients $(p \times 1)$ 
The coefficients in the linear predictor for the $\mathrm{CPH}$ model are estimated via the Maximum Partial Likelihood Estimator in the logarithm of the linear predictor (HARRELL, 2015). Similar to the logistic regression, the coefficients in the linear form are called log hazards. To provide a better interpretation, some studies opt to calculate the Hazard Ratio (HR), which is a measure of association between the predictor and the response. The Hazard Ratio is obtained as the exponential of the $\log$ hazard and corresponds to the average rate of change in the risk of the event to occur in time with respect to the independent variable $X$ (HARRELL, 2015; KLEINBAUM; KLEIN, 2012). An $H R=1.00$ corresponds to a log hazard $=0$, indicating that $X$ does not influence in $Y$. Therefore, if the $H R>1.00$, the hazard for $Y$ to occur are increased in $X$; and if the $H R<1.00$ the hazard are decreased.

The settings of each model application and additional assumptions are described thoroughly in each project from Sections 2.2 and Chapters 3 to 8 .

\section{2 \\ Thesis projects and data science applications}

Each research question was addressed as a Data Science project in this thesis, following the phases described in Section 2.1. In addition to the definitions, we include general settings that pertained to all projects, described as follows:

1) Business Understanding (Hypothesis generation): In the first stage of the process, we generated the research questions and goals of the study. We also listed potential variables and their sources required for achieving the expected results.

2) Data understanding and preparation (Data collection and processing): Studies were observational, and data sources were retrospective, using convenience sampling. Different data sources were used, which required thorough preparation, data recovery when possible, and linking of databases. For public data, we informed the corresponding data source location. Private data could not be shared but can be provided under request. We also reported the data selection criteria, such as patients and units, also named "Study Population", following the study's objectives. For all studies, an ethics statement was provided. We highlight that data 
used in the studies were anonymized or de-identified following the Brazilian General Regulation of Data Protection (Lei No 13.709, de 14 de Agosto de 2018)

3) Modeling (Statistical Analysis): We described the analyzed population using descriptive statistics. According to the study's objective, different statistical methods were used, ranging from regression models to regularization. We conducted sensitivity analyses that included intermediate, additional, or subgroup analysis and imputation of missing values to evaluate the robustness of the results. Most of the analyses were performed using the $\mathrm{R}$ programming language (R CORE TEAM, 2021)

4) Evaluation (Interpretation of the results): We reported the main findings and model outputs using tables and figures. Results were then discussed separately, considering the main findings' statistical and clinical interpretation. We note that one or more healthcare specialists reviewed the studies under the context of healthcare data.

5) Deploy (Reporting and sharing evidence): The studies were mainly reported as research articles. Additional analyses were compiled into supplementary materials for those articles. Codes and data were shared whenever possible at a GitHub Repository (https://github.com/lslbastos) - we provided the sources' locations for public data and code for data preparation and statistical analysis. Articles already published also contain more specific information on the data sources.

The data science projects resulted in six research articles that summarize and compile all the research questions. A summary of the articles is presented in Table 2.1 , and we provide a brief description of the data sources, methods, and findings.

1) Article 1 - "Structure and process associated with the efficiency of intensive care units in low-resource settings: An analysis of the CHECKLIST-ICU trial database": In this study, we aimed to identify organizational characteristics associated with ICU efficiency. We used data from the CHECKLIST-ICU clinical trial - 13,635 adult ICU patients in 118 units in Brazil, with 63 potential organizational characteristics (structure and process) to be evaluated. To define ICU efficiency, we calculated risk-adjusted metrics, the SMR and the SRU and identified two efficiency groups (efficient vs non- 
efficient units). We used a multivariable logistic regression model with adaptive elastic-net regularization (AENET). AENET combines the LASSO

(Least Absolute Shrinkage and Selection Operator, L1-norm) and the ridge (L2norm) penalizations with weights to provide consistent estimators. Compared to the traditional stepwise approach to select variables and infer associations, the AENET model can include all variables of interest and provide more reliable estimates. We Identified 47 efficient and 71 non-efficient units and conduct surveillance of nosocomial infection rates, and the assessment of infection control was associated with efficiency.

Table 2.1 - Summary of research studies

\begin{tabular}{|c|c|c|c|c|}
\hline $\begin{array}{l}\text { Research } \\
\text { Study }\end{array}$ & Research question & Objectives & $\begin{array}{l}\text { Study } \\
\text { Participants/Data }\end{array}$ & Methods \\
\hline $\begin{array}{l}\text { Article 1: } \\
\text { Bastos et } \\
\text { al. (2020) } \\
\text { Published - } \\
\text { Journal of } \\
\text { Critical Care }\end{array}$ & $\begin{array}{l}\text { What are the } \\
\text { organizational } \\
\text { characteristics that } \\
\text { drive efficiency in } \\
\text { ICUs under low- } \\
\text { resource settings? }\end{array}$ & $\begin{array}{l}\text { To identify structure } \\
\text { and process } \\
\text { characteristics } \\
\text { associated with ICU } \\
\text { efficiency }\end{array}$ & $\begin{array}{l}118 \text { ICUs; } 13,635 \\
\text { ICU admissions; } \\
\text { CHECKLIST-ICU } \\
\text { Trial }\end{array}$ & $\begin{array}{l}\text { - ICU benchmarking with } \\
\text { Rapoport-Teres } \\
\text { efficiency matrix. } \\
\text { - Adaptative Elastic-net } \\
\text { multivariable logistic } \\
\text { regression to identify } \\
\text { associations }\end{array}$ \\
\hline $\begin{array}{l}\text { Article 2: } \\
\text { Bastos \& } \\
\text { Wortel et } \\
\text { al. (2021)* } \\
\text { Ongoing } \\
\text { article }\end{array}$ & $\begin{array}{l}\text { What are the } \\
\text { advantages and } \\
\text { disadvantages of } \\
\text { the continuous and } \\
\text { categorical } \\
\text { combinations of } \\
\text { SMR and SRU for } \\
\text { ICU } \\
\text { benchmarking? }\end{array}$ & $\begin{array}{l}\text { To compare the use } \\
\text { of categorical and } \\
\text { continuous } \\
\text { approach to } \\
\text { combine SMR and } \\
\text { SRU for } \\
\text { benchmarking ICU } \\
\text { performance }\end{array}$ & $\begin{array}{l}\text { ORCHESTRA } \\
\text { study data (Brazil) } \\
134 \text { ICUs and over } \\
282,000 \\
\text { admissions } \\
\text { (2016-2019) } \\
\text { NICE registry (The } \\
\text { Netherlands) } \\
83 \text { ICUs over } \\
164,000 \\
\text { admissions } \\
\text { (2016-2019) }\end{array}$ & $\begin{array}{l}\text { - Efficiency matrix } \\
\text { - Regression analysis } \\
\text { and correlation in } \\
\text { different settings } \\
\text { (Brazilian and Dutch } \\
\text { ICUs) } \\
\text { List the advantages and } \\
\text { disadvantages of } \\
\text { continuous and } \\
\text { categorical approaches. }\end{array}$ \\
\hline $\begin{array}{l}\text { Article 3: } \\
\text { Bastos \& } \\
\text { Wortel et } \\
\text { al. (2021)* } \\
\text { Ongoing } \\
\text { article }\end{array}$ & $\begin{array}{l}\text { What are the } \\
\text { organizational } \\
\text { characteristics that } \\
\text { drive efficiency in } \\
\text { ICUs? }\end{array}$ & $\begin{array}{l}\text { To identify the } \\
\text { organizational } \\
\text { factors associated } \\
\text { with efficiency in } \\
\text { ICUs considering } \\
\text { potential } \\
\text { confounders. }\end{array}$ & $\begin{array}{l}\text { ORCHESTRA } \\
\text { study data (Brazil) } \\
134 \text { ICUs and over } \\
284,000 \\
\text { admissions } \\
(2016-2019)\end{array}$ & $\begin{array}{l}\text { Defining confounder } \\
\text { variables } \\
\text { - Estimating average } \\
\text { treatment effects using } \\
\text { causal random forests } \\
\text { (CRF), a machine } \\
\text { learning algorithm for } \\
\text { causal inference } \\
\text { - }\end{array}$ \\
\hline $\begin{array}{l}\text { Article 4: } \\
\text { Ranzani \& } \\
\text { Bastos et } \\
\text { al. (2021)* } \\
\text { Published - } \\
\text { The Lancet } \\
\text { Respiratory } \\
\text { Medicine }\end{array}$ & $\begin{array}{l}\text { What are the } \\
\text { characteristics and } \\
\text { outcomes of } \\
\text { COVID-19 hospital } \\
\text { admissions in } \\
\text { Brazil? }\end{array}$ & $\begin{array}{l}\text { To describe the } \\
\text { course of the first } \\
250,000 \text { COVID-19 } \\
\text { admissions } \\
\text { (characteristics, use } \\
\text { of intensive care, } \\
\text { respiratory support, } \\
\text { and outcomes) in } \\
\text { Brazil. }\end{array}$ & $\begin{array}{l}254,288 \text { COVID-19 } \\
\text { hospital } \\
\text { admissions, in five } \\
\text { macro-regions of } \\
\text { Brazil; SIVEP- } \\
\text { Gripe (Influenza } \\
\text { Epidemiological } \\
\text { Surveillance } \\
\text { Information } \\
\text { System) }\end{array}$ & $\begin{array}{l}\text { - Descriptive statistics } \\
\text { stratified by patient's } \\
\text { demographics, Brazilian } \\
\text { macro-regions, and type } \\
\text { of resource (ICU and } \\
\text { respiratory support) }\end{array}$ \\
\hline $\begin{array}{l}\text { Article 5: } \\
\text { Bastos \& } \\
\text { Ranzani et } \\
\text { al. (2021)* } \\
\text { Published - } \\
\text { The Lancet } \\
\text { Respiratory } \\
\text { Medicine }\end{array}$ & $\begin{array}{l}\text { What are the major } \\
\text { differences in } \\
\text { severity, use of } \\
\text { resources and } \\
\text { outcomes between } \\
\text { the first and } \\
\text { second COVID-19 } \\
\text { waves? }\end{array}$ & $\begin{array}{l}\text { To compare the first } \\
\text { and second wave of } \\
\text { COVID-19 hospital } \\
\text { admissions in Brazil } \\
\text { regarding severity, } \\
\text { use of resources } \\
\text { (ICU and respiratory } \\
\text { support) and } \\
\text { outcomes. }\end{array}$ & $\begin{array}{l}1,217,332 \text { COVID- } \\
19 \text { hospital } \\
\text { admissions, in five } \\
\text { macro-regions of } \\
\text { Brazil; SIVEP- } \\
\text { Gripe (Influenza } \\
\text { Epidemiological } \\
\text { Surveillance } \\
\text { Information } \\
\text { System) }\end{array}$ & $\begin{array}{l}\text { - Descriptive statistics } \\
\text { stratified by patient's } \\
\text { demographics and use } \\
\text { of resources } \\
\text { - Comparison between } \\
\text { two time periods } \\
\text { - Development of an } \mathrm{R} \\
\text { shiny app for monitoring } \\
\text { of hospital admission }\end{array}$ \\
\hline
\end{tabular}




\begin{tabular}{|c|c|c|c|c|}
\hline $\begin{array}{l}\text { Article 6: } \\
\text { Kurtz \& } \\
\text { Bastos et } \\
\text { al. (2021)* } \\
\text { Published - } \\
\text { Intensive } \\
\text { Care } \\
\text { Medicine }\end{array}$ & $\begin{array}{l}\text { What are the } \\
\text { factors associated } \\
\text { with evolving } \\
\text { changes in } \\
\text { mortality for } \\
\text { COVID-19 ICU } \\
\text { admissions? }\end{array}$ & $\begin{array}{l}\text { To analyze the } \\
\text { dynamic of COVID- } \\
19 \text { ICU admissions: } \\
\text { characteristics, use } \\
\text { of respiratory } \\
\text { support and } \\
\text { differences in } 60 \text { - } \\
\text { day in-hospital } \\
\text { mortality }\end{array}$ & $\begin{array}{l}126 \text { ICUs; 13,301 } \\
\text { COVID-19 ICU } \\
\text { admissions; Data } \\
\text { from the Rede } \\
\text { D'Or São Luiz } \\
\text { (Epimed Solutions) }\end{array}$ & $\begin{array}{l}\text { - Descriptive statistics } \\
\text { stratified by patient's } \\
\text { demographics. } \\
\text { - Identification of } \\
\text { breakpoints periods of } \\
\text { change in the structure } \\
\text { of time series. } \\
\text { - Survival analysis for } \\
60 \text {-day mortality using } \\
\text { random-effects Cox } \\
\text { multivariable regression }\end{array}$ \\
\hline
\end{tabular}

2) Article 2: This proposed study aims to evaluate whether combining riskadjusted metrics such as the SMR and SRU using a categorical or continuous approach is more suitable for ICU performance evaluation. SMR and SRU are widely used to measure ICU performance or efficiency, and most studies benchmark them with Rapoport-Teres' efficiency matrix, thus obtaining four groups. Recently, a study proposed a continuous combination by using the average of SMR and SRU $\left(\frac{S M R+S R U}{2}\right)$. Although the continuous metric provide statistical properties, it must be validated in the settings considered for the study since the association between SMR and SRU may impact the resulting metric. The advantages and disadvantages of using a categorical or continuous combination of SMR and SRU are uncertain for ICU benchmarking. As methods, we used statistical modelling to evaluate the distribution of those combinations in two sets of ICU data, one from Brazil and the other from The Netherlands.

3) Article 3: This proposed study follows the results from Article 2. We considered one of the efficiency modeling approaches identified as best suited for benchmarking for the Brazilian ICUs. Then, using a causal inference approach, we identified those organizational factors associated with increased performance in intensive care units. We proposed the Causal Random Forests (CRF), a causal machine learning method based on the Random Forest but adapted to estimate the average treatment effect. Compared to regression models, CRF has the advantage of being nonparametric and can incorporate potential nonlinear relationships between the treatment variable and the outcomes, adjusted by the potential confounders.

4) Article 4 - "Characterisation of the first 250000 hospital admissions for COVID-19 in Brazil: a retrospective analysis of nationwide data": In this 
study, we provided a thorough description of the initial COVID-19 hospital admissions in Brazil. We used data from adult patients with COVID-19 confirmed by RT-PCR (registered in the national surveillance system, the SIVEP-Gripe, the main notification database for COVID-19 cases and hospital admissions and other data sources. Along with an extensive linking of different datasets, we mainly used descriptive statistical methods to estimate the total burden of hospital admissions per population and in-hospital mortality for the whole country and stratified by region, age, sex, sociodemographic conditions, and use of resources (ICU and respiratory support). We evaluated the robustness of estimates by including analyses of patients diagnosed by other testing criteria and imputing missing data using Multiple Imputation by Chained Equations (MICE). From February 16, 2020, to August 15, 2020, 258,288 hospital admissions occurred. Temporally, the first cases were confirmed in the Southeast region. There was an increase of cases in the North region, followed to the Northeast and the South and Central-west regions. 232,036 were admissions with an outcome, from which overall in-hospital mortality was $38 \%$, $59 \%$ for those who required intensive care, and $79 \%$ for those invasively ventilated. The use of resources and outcomes also varied within regions; for instance, North and Northeast regions, which have the lowest number of ICU beds, mainly were impacted by the pandemic, compared to South and Southeast regions, those with the highest bed availability. Mortality was also increased for patients aged under 60 years old. Our results showed that vulnerable healthcare systems were heavily affected by the pandemic, showing potential collapse and high mortality. This study was one of the first analyses on a large dataset of COVID-19 patients nationwide and consisted of documentation of this context. We reinforce that this study was used as the basis for new documents and strategies for mitigating and controlling the pandemic for policymakers and other institutions after its publication.

5) Article 5 - "Severity, resource use and outcomes of COVID-19 hospital admissions in Brazil: a comparison between the first and second wave". This study is a short, updated analysis of Article 4. This article included adult hospital admissions registered in the SIVEP-Gripe database from February 16, 2020, to May 24, 2021. We used descriptive statistics and calculated estimates for resources utilization and outcomes stratified by age and respiratory support. 
1,217,332 hospital admissions were analyzed. In this period, Brazil showed a second surge of the COVID-19 pandemic, with more admissions per week, more patients with severe symptoms than the first wave (mostly depicted in Article 4), and increased in-hospital mortality among those that underwent respiratory support. This second wave was also present in the context of new Variants of Concern in the country and large variation of mobility pattern indicating low adherence to non-pharmacological. This study was published as a Correspondence/short paper to inform the pandemic situation and highlight the need for urgent actions to control the pandemic. A dashboard was created as an $R$ shiny app to provide updated information on the course of the pandemic (https://lslbastos.shinyapps.io/sivep_covid_brazil/) and assist the decisionmakers and other researchers.

6) Article 6 - "Evolving changes in mortality of 13,301 critically ill adult patients with COVID-19 over 8 months". Throughout the pandemic, the profile of patients and use of resources dynamically changes, which may impact mortality. This study aimed to analyze the association of clinical profiles and respiratory support to ICU patients' mortality changes. We included data from patients admitted between February $26^{\text {th }}, 2020$, to October $28^{\text {th }}, 2020$, at 126 ICUs from a private Brazilian hospital network (Rede D'Or São Luiz). To assess temporal changes in the pandemic progressions, we identified breakpoint periods by evaluating structural changes in the time series of daily number of deaths. Then, we used a random-effects multivariable Cox regression model to estimate the association of the initial respiratory support, adjusted by clinical profiles and admission periods, with 60-day in-hospital mortality. To account for the nonrandomization of data, we used with inverse probability weighting method. We identified 13,301 COVID-19 ICU admissions, with a 60-day inhospital mortality of $13 \%$ and $58 \%$ for those who underwent invasive mechanical ventilation. We identified four distinct periods: mortality was high initially and decreased in the last periods. Also, the use of noninvasive respiratory support and increased throughout the period. We observed an increase in the number of beds as the number of admissions arose. Finally, we identified that noninvasive respiratory support was associated with survival after adjusting for clinical profiles with the Cox regression model. This study complements the findings from Article 4, showing the perspective ICU setting 
different from the national or average Brazilian setting, thus indicating that adequate preparedness and high availability of resources may improve the outcomes during the pandemic. 


\title{
Article 1 - Structure and Process Associated with the Efficiency of Intensive Care Units in Low-Resource Settings: An Analysis of the CHECKLIST-ICU Trial database
}

This article was published at the Journal of Critical Care

\begin{abstract}
Purpose: Characteristics of structure and process impact ICU performance and the outcomes of critically ill patients. We sought to identify organizational characteristics associated with efficient ICUs in low-resource settings.

Materials and Methods: This is a secondary analysis of a multicenter clusterrandomized clinical trial in Brazil (CHECKLIST-ICU). Efficient units were defined by standardized mortality ratio (SMR) and standardized resource use (SRU) lower than the overall medians and non-efficient otherwise. We used a regularized logistic regression model to evaluate associations between organizational factors and efficiency.
\end{abstract}

Results: From 118 ICUs (13,635 patients), 47 units were considered efficient and 71 non-efficient. Efficient units presented lower incidence rates (median[IQR]) of central line-associated bloodstream infections (4.95[0.00-22.0] vs 6.29[0.00-25.6], $\mathrm{p}=.04)$, utilization rates of mechanical ventilation $(0.41[0.07-0.73]$ vs $0.58[0.19$ $0.82], \mathrm{p}<.001)$, central venous catheter $(0.67[0.15-0.98]$ vs $0.78[0.33-0.98]$, $\mathrm{p}=0.04)$, and indwelling urinary catheter $(0.62[0.22-0.95]$ vs $0.76[0.32-0.98]$, $\mathrm{p}<0.01)$ than non-efficient units. The reported active surveillance of ventilatorassociated pneumonia $(\mathrm{OR}=1.72 ; 95 \% \mathrm{CI}, 1.16-2.57)$ and utilization of central venous catheters $(\mathrm{OR}=1.94 ; 95 \% \mathrm{CI}, 1.32-2.94)$ were associated with efficient ICUs.

Conclusions: In low-resource settings, active surveillance of nosocomial infections and the utilization of invasive devices were associated with efficiency, supporting the management and evaluation of performance indicators as a starting point for improvement in ICU. 
Keywords: Intensive care; ICU organization; ICU benchmarking; Quality indicators; Organizational characteristics

Abbreviations: AENET, Adaptive Elastic-Net; CI, Confidence Interval; CLABSI, Central-line associated bloodstream infection; CVC, Central Venous Catheter; ICU, Intensive care unit; IQR, Interquartile Range; MV, Mechanical Ventilator; RASS, Richmond Agitation-Sedation Scale; SAPS, Simplified Acute Physiology Score; SMR, Standardized Mortality Ratio; SRU, Standardized Resource Use; UTI, Urinary tract infection; VAP, Ventilator-Associated Pneumonia.

\section{1 \\ Introduction}

The evaluation of intensive care unit (ICU) performance has been a demand from society and funders to ensure the optimal use of resources and better patient outcomes. This assessment provides potential "actionable indicators" that can assist the management and improvements (DE LANGE; DONGELMANS; DE KEIZER, 2017) in areas such as risk-adjusted mortality, patient safety, processes of care, costs, and patient satisfaction (DONABEDIAN, 1988; GARLAND, 2005; SALLUH; SOARES; KEEGAN, 2017; WOODHOUSE et al., 2009). ICU efficiency has been mostly evaluated in two domains: mortality and resource use. Also, the determinants of ICU efficiency are especially critical in low- and middleincome countries (LMIC), where access to intensive care is limited (BOZZA; SALLUH, 2010), and outcomes remain suboptimal (SALLUH; SOARES; SINGER, 2017).

Besides the patients' severity-of-illness, variability in ICU clinical outcomes, and its efficiency is also related to the structure and processes of care (CHECKLEY et al., 2014). Efficient ICUs were defined by the presence of daily clinical rounds and the presence of an emergency department (ROTHEN et al., 2007). Units with poor mortality outcomes have increased staff occupancy rates, after-hours discharges, and lower staffing levels (MCCLEAN et al., 2017). In Brazil, previous studies showed that better mortality and resource use rates were associated with an increased number of care protocols (SOARES et al., 2015), and the low availability of resources was related to poor mortality outcomes for sepsis (MACHADO et al., 2017). 
Little has been shown on what organizational characteristics are determinants of efficiency in LMIC (MACHADO et al., 2017; SOARES et al., 2015, 2017). Also, previous analyses only considered a more organized sample of units that spontaneously adhered to a commercial benchmarking database (SOARES et al., 2015). Therefore, our study sought to identify structure and process characteristics associated with efficiency using data from the CHECKLIST-ICU trial (CAVALCANTI et al., 2016), a large contemporary multicenter randomized trial that tested a quality improvement intervention on a variety of units with different organizational aspects.

\section{2 \\ Materials and Methods}

\subsection{1}

\section{Study design and data source}

This study is a secondary observational analysis of a cluster-randomized trial testing the effect of a multifaceted quality improvement intervention in mortality outcomes (the CHECKLIST-ICU Trial, ClinicalTrials.gov Identifier: NCT01785966) (CAVALCANTI et al., 2016). Detailed information on this Trial has been published previously (CAVALCANTI et al., 2016; DAMIANI et al., 2015). Briefly, the trial was conducted in two phases, an observational (August 2013 - March 2014) and a randomized (April 2014 - November 2014) phase with two parallel groups. The Trial did not consider patients with a high likelihood of early death before 72-hours of ICU stay, those receiving exclusive palliative care, and those with suspected or confirmed diagnosis of brain death (CAVALCANTI et al., 2016).

\section{2 .2}

\section{Study population}

As the effect of the CHECKLIST-ICU intervention was not statistically significant, we considered all 13,635 adult patients from 118 ICUs in our analysis. Thus, the median number of patients included per unit in the trial was 120 (IQR: 119-120), with a minimum of 64 patients and a maximum of 136. 
In our analysis, we considered the patient's Simplified Acute Physiology Score (SAPS-3) at admission, the discharge status at the hospital, the ICU lengthof-stay provided in the CHECKLIST-ICU database. We also included the trial's secondary exploratory outcomes of clinical results and processes-of-care to our analysis (CAVALCANTI et al., 2016; DAMIANI et al., 2015): days on a mechanical ventilator; ventilator-free days in a 28-day period; and infection events regarding ventilator-associated pneumonia (VAP), central-line bloodstream infection (CLABSI), and urinary tract infection (UTI); and the information of seven care processes: head-of-bed elevated at $30^{\circ}$ or more; moderate to light sedation or alert and calm (Richmond Agitation-Sedation Scale score [RASS], -3 to 0); mechanical ventilation tidal volume $8 \mathrm{~mL} / \mathrm{kg}$ of predicted body weight or less; venous thromboembolism (VTE) prophylaxis; central venous catheter use (CVC); and indwelling urinary catheter use and mechanical ventilator (MV) use.

The primary ICU data comprised reported characteristics of the unit regarding its structure and processes based on the recommendations from the Brazilian Health Surveillance Agency (DAMIANI et al., 2015). Those aspects consisted of general information on the ICU and hospital and the organizational characteristics in human resources, health care resources, infrastructure, equipment, availability of care protocols, surveillance of quality-of-care measures, transport of patients, prevention of health care related infections, risk management, and family policies (DAMIANI et al., 2015)

\section{2 .3}

\section{Outcomes and ICU efficiency}

Our primary outcome was the efficiency of ICU units, which was mainly evaluated in two domains: mortality and resource use.

We considered the standardized mortality ratio (SMR) and standardized resource use (SRU) (ROTHEN et al., 2007). The first was defined as the observed number of deaths divided by the expected number of deaths for each ICU using the SAPS-3 standard mortality equation (MORENO et al., 2005). To obtain a proper calibration of the SAPS-3 risk model, we conducted the first-level customization, which diminished the over- or underestimation of the predicted mortality. We evaluated the recalibrated model with the calibration belt technique (FINAZZI et al., 2011; POOLE et al., 2012) (for more details, see Appendix A1.1). SRU was 
defined as the ratio of observed-to-expected resource use (ROTHEN et al., 2007). We used the ICU-LOS as a surrogate variable to measure resource utilization (NEWGARD et al., 2010; ROTHEN et al., 2007), and to reduce potential miscalibration, we estimated the expected resource use denominator from the CHECKLIST-ICU database (for more details see Appendix A1.2).

We defined efficiency based on the Efficiency Matrix method (ROTHEN et al., 2007; SALLUH; SOARES; KEEGAN, 2017): units with SMR and SRU values lower than their respective overall medians as the efficient units, and non-efficient otherwise. We refer to those classifications as "efficiency groups."

We obtained information on structure and process from the CHECKLISTICU data (CAVALCANTI et al., 2016; DAMIANI et al., 2015), which comprehended the reported presence of a specific process-of-care, organizational practices, or resources in the ICU. Information was retrieved from a questionnaire filled by the unit's representative (for more information, see CAVALCANTI et al. (2016) and DAMIANI et al. (2015)). Since the reported presence was in a "Yes/No" format, we considered only those characteristics applicable to all units and in which there was at least one positive response. From the 63 organizational characteristics, 50 were deemed eligible (for more details, see Appendix A1.4). Our study sought to evaluate the association between these organizational aspects with ICU efficiency.

Our secondary outcomes were the CHECKLIST-ICU's exploratory secondary outcomes of clinical results and processes-of-care. As in the Trial (CAVALCANTI et al., 2016; DAMIANI et al., 2015), we calculated the number of patient-days using the invasive device over the total number of patient-days for each process-of-care. For clinical results, we obtained the number of VAP, CLABSI, and UTI events over the total number of patient-days on MV, CVC, and indwelling urinary catheter, respectively, as well as the number of days in moderate to light sedation or alert and calm (Richmond Agitation-Sedation Scale score [RASS], -3 to 0 ) and venous thromboembolism (VTE) prophylaxis over the total number of patient-days, and the total patient-days of mechanical ventilation tidal volume 8 $\mathrm{mL} / \mathrm{kg}$ of predicted body weight or less over the number of MV days. We calculated these rates for each unit and evaluated their variability within the efficiency groups. 


\subsection{4}

\section{Statistical analysis}

We described for the efficiency groups the median and interquartile range (IQR) for quantitative variables, frequency, and proportions for categorical variables, when appropriate. We used the two-sided Wilcoxon signed-rank test and Fisher's exact test to evaluate differences between efficiency groups. Therefore, we analyzed the general characteristics and the secondary outcomes of clinical results and process-of-care.

Furthermore, we evaluated individual associations of each organizational characteristic in a univariate analysis using the mentioned statistical tests. For the multivariable analysis, we selected those characteristics with $\mathrm{p}<0.15$ in the statistical tests as the independent variables. We evaluated the strength of their association by calculating Cramer's V coefficient (KOTRLIK; WILLIAMS, 2003). The response variable corresponded to the efficiency groups.

We used the adaptive elastic-net (AENET, ZOU; ZHANG, 2009) in the multivariable logistic regression. This model is a regularization method that combines L1 and L2 norms (BARRETT; LOCKHART, 2019) to penalize regression coefficients, which allows the inclusion of a large set of independent variables, and group those with some degree of collinearity (BARRETT; LOCKHART, 2019; HASTIE; TIBSHIRANI; FRIEDMAN, 2009; HEINZE; WALLISCH; DUNKLER, 2018). The effects were evaluated with odds ratio and 95\% confidence interval (Details on the model estimation procedure are in Appendix A1.5).

All of the analyses were performed in R 3.6.0, with RStudio 1.2.1335, using the dplyr and ggplot2 packages from tidyverse for data manipulation and visualization, givitiR (NATTINO et al., 2017a) for fitting the calibration belts, and the glmnet (FRIEDMAN; HASTIE; TIBSHIRANI, 2010) and msaenet (XIAO; XU, 2015) packages for AENET model. 


\section{3}

\section{Results}

\subsection{1}

\section{Efficiency groups and general characteristics}

The SMR and SRU of the ICUs are depicted in the efficiency matrix (Figure 3.1), which also discriminates the efficiency groups.

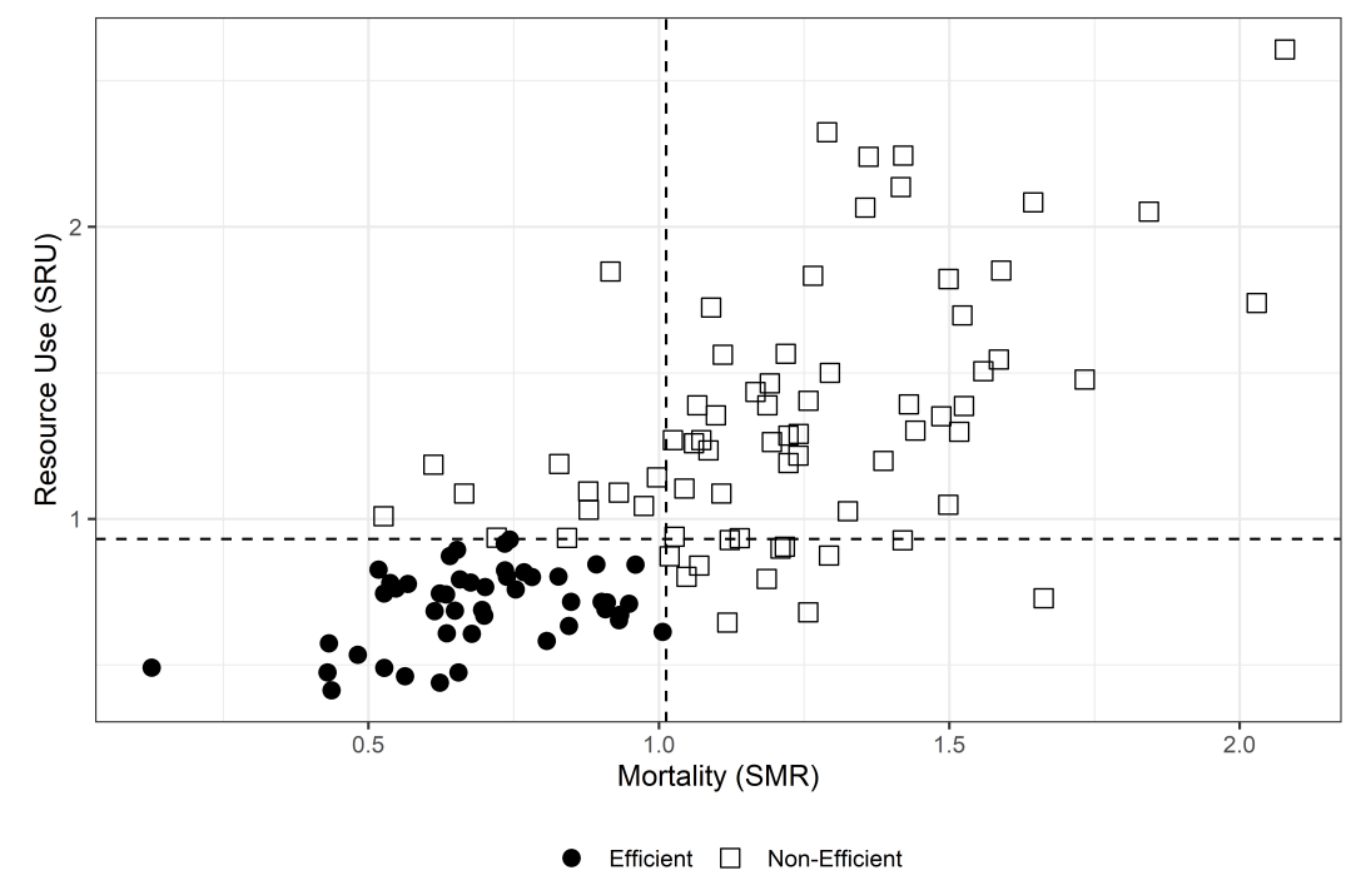

Figure 3.1 - Efficiency matrix and the efficiency groups.

Axis corresponding to the performance indicators: the standardized mortality ratio (SMR) and standardized resource use (SRU). Reference lines are the overall median $S M R=1.01$ and the overall median SRU $=0.93$. The efficient units are those with both SMR and SRU lower than their corresponding medians, and non-efficient otherwise. From 118 units, 47 were classified as efficient and 71 as non-efficient.

The overall median SRU and median SMR were 1.01 (IQR, [0.71-1.15]) and 0.93 (IQR, [0.74-1.34]), respectively, and the median SAPS-3 was 51 (IQR, [4064]). The efficient group included 47 units, whereas the remaining 71 units were in the non-efficient group (A list with all units and their corresponding performance indicators is Appendix A3.3). The performance indicators and case-mix values were different between the groups $(\mathrm{p}<0.001)$. There was a strong linear correlation between the SMR and SRU (Pearson's correlation test: 0.73; 95\% CI, 0.64-0.81). 
The general characteristics of the ICUs, according to the efficiency groups, are shown in Table 3.1.

Table 3.1- General characteristics of all ICUs the efficiency groups.

Characteristics of the ICUs within the performance groups. The number of ICUs from the efficient and non-efficient performance groups are reported with the percentage of the total size of each performance group and the median (IQR) for hospital and ICU beds. P-values were calculated using Fisher's exact test for the categorical variables and the Wilcoxon signed-rank test for the quantitative variables. SMR and SRU were expected to differ between groups due to the classification criteria. Although the test rejected the hypothesis of differences in the SAPS-3 median, this distinction was in 2 points.

\begin{tabular}{|c|c|c|c|c|}
\hline Characteristic & $\begin{array}{c}\text { Total } \\
(\mathrm{n}=118)\end{array}$ & $\begin{array}{l}\text { Efficient } \\
(\mathrm{n}=47)\end{array}$ & $\begin{array}{c}\text { Non-efficient } \\
(\mathrm{n}=71)\end{array}$ & $p$ \\
\hline SMR, median (IQR) & $1.01(0.71-1.25)$ & $0.68(0.59-0.82)$ & $1.22(1.06-1.42)$ & $<.001$ \\
\hline SRU, median (IQR) & $0.93(0.74-1.34)$ & $0.71(0.61-0.80)$ & $1.27(1.03-1.53)$ & $<.001$ \\
\hline SAPS-3, median (IQR) & $51(40-64)$ & $52(41-65)$ & $50(39-63)$ & $<.001$ \\
\hline Hospital complexity, no. (\%) & & & & 0.06 \\
\hline Primary & $2(2)$ & $0(0)$ & $2(3)$ & \\
\hline Secondary & $24(20)$ & $14(30)$ & $10(14)$ & \\
\hline Tertiary & $92(78)$ & $33(70)$ & $59(83)$ & \\
\hline Hospital type, no. (\%) & & & & 0.18 \\
\hline General & $101(86)$ & $43(92)$ & $58(82)$ & \\
\hline Specialized & $17(14)$ & $4(8)$ & $13(18)$ & \\
\hline Hospital beds, median (IQR) & $214(131-344)$ & $157(97-294)$ & $237(150-350)$ & 0.01 \\
\hline ICU beds, median (IQR) & $12(10-20)$ & $12(10-22)$ & $12(10-20)$ & 0.96 \\
\hline ICU type, no. (\%) & & & & 0.05 \\
\hline Mixed & $93(79)$ & $38(81)$ & $55(77)$ & \\
\hline Medical & $13(11)$ & $8(17)$ & $5(7)$ & \\
\hline Surgical & $5(4)$ & $0(0)$ & $5(7)$ & \\
\hline Specialized & $7(6)$ & $1(2)$ & $6(9)$ & \\
\hline
\end{tabular}

ICU: Intensive Care Unit

IQR: Interquartile Range ( $1^{\text {st }}$ Quartile $-3^{\text {rd }}$ Quartile)

SAPS-3: Simplified Acute Physiology Score - version 3

SMR: Standardized Mortality Ratio

SRU: Standardized Resource Use

Most of the ICUs were in tertiary and general hospitals, without significant differences between the efficient and non-efficient ICUs. However, the efficient group was mostly composed of ICUs from hospitals with a smaller number of beds (Median: 157 [IQR, 97-294]) than the non-efficient ICUs (Median: 237 [IQR, 150350]). The median number of ICU beds was similar between the performance groups. The type of ICUs differed between the efficient and non-efficient groups ( $p$ 
$=0.05$ ), being with a large proportion of mixed ICUs in both groups. However, surgical and specialized units were mostly present within the non-efficient ICUs.

\subsection{2}

\section{Adherence to processes of care and clinical results}

The secondary exploratory clinical outcomes of adherence to processes of care and clinical results within each performance group are presented in Table 3.2.

Compared to the non-efficient units, the efficient ICUs had lower median utilization rates of MV use (0.41 [IQR, 0.07-0.73] vs 0.58 [IQR, 0.19-0.82]; p < 0.001), CVC use (0.67 [IQR, 0.15-0.98] vs 0.78 [IQR, 0.33-0.98]; $\mathrm{p}=0.02$ ), and indwelling urinary catheter use (0.62 [IQR, 0.22-0.95] vs 0.76 [IQR, 0.32-0.98]; p $<0.01)$. There was also a high median rate of adequate prophylaxis for VTE in the efficient ICUs (0.83 [IQR, 0.57-0.99] vs 0.73 [IQR, 0.16-0.96]; $\mathrm{p}<0.001)$.

Regarding the clinical outcomes, the CLABSI incidence density was lower in the efficient ICUs (4.95 [IQR, 0.00-22.0]) compared to the non-efficient units (6.29 [IQR, 0.00-25.6]; $\mathrm{p}=0.04)$. The number of days on mechanical ventilation and average ventilator-free days in a 28-day period were lower in the efficient units than in the non-efficient ICUs. The utilization rates of VAP (3.11 [IQR, 0.00-11.2] vs 3.56 [IQR, 0.00-14.0]; $\mathrm{p}=0.33$ ) or UTI (6.93 [IQR, 0.00-19.2] vs 8.47 [IQR, 0.00$36.8] ; \mathrm{p}=0.37$ ) did not present relevant differences between the groups.

\subsection{3}

\section{Association of structure and process with the efficiency groups}

Finally, we evaluated the association of the characteristics regarding structure and process with the efficiency groups. In the univariable analysis (for more details see Appendix A1.4), we verified that the efficient ICUs had an association to the practice of assessing the mechanical ventilation utilization rate $(\mathrm{OR}, 3.47 ; 95 \% \mathrm{CI}$, 1.29-10.51) and central venous catheter utilization rate (OR, 4.66; 95\% CI, 1.6715.2).

Similarly, the efficient units also had a higher proportion of reported positive responses to the practice of monitoring ventilator-associated pneumonia (VAP) incidence density (OR, 5.09; 95\% CI, 1.56-21.86), central line-associated bloodstream infection (CLABSI) incidence density (OR, 3.26; 95\% CI, 1.0712.09), and urinary tract infection (UTI) incidence density (OR, 2.72; 95\% CI, 1.00- 
8.3). Efficiency units also presented some association with the reported evaluation of 24-hour readmission rate (OR, 2.00; 95\% CI 0.89); and the routine of registering ICU adverse events (OR, 1.95; 95\% CI, 0.85-4.59).

Table 3.2 - Efficiency groups and secondary outcomes of adherence to processes of care and clinical results

Results from the secondary outcomes of adherence to processes of care and clinical results of patients during the CHECKLIST intervention execution period. We calculated those metrics for each ICU and provided information regarding the variability of the units in each efficiency group. We tested the differences using the Wilcoxon signed-rank test. In process-of-care, the utilization rates of mechanical ventilation, central venous catheter, and indwelling urinary catheter were lower in efficient units compared to the non-efficient groups. The result was similar regarding the CLABSI incidence density rates, and the average number of ventilator days and ventilator-free days.

\begin{tabular}{|c|c|c|c|}
\hline Metric & $\begin{array}{l}\text { Efficient } \\
(\mathrm{n}=47)\end{array}$ & $\begin{array}{c}\begin{array}{c}\text { Non-efficient } \\
(\mathrm{n}=71)\end{array} \\
\end{array}$ & $\mathrm{P}$ \\
\hline \multicolumn{4}{|c|}{ Processes of care, no. of patient-days used/total no. of patient-days, median (IQR) } \\
\hline Head of bed elevated $\geq 30^{\circ}$ & $0.99(0.75-1.00)$ & $0.99(0.38-1.00)$ & .30 \\
\hline Adequate prophylaxis for venous thromboembolism (VTE) & $0.83(0.57-0.99)$ & $0.73(0.16-0.96)$ & $<.001$ \\
\hline Moderate sedation to alert and calm (RASS -3 to 0 ) & $0.39(0.02-0.71)$ & $0.34(0.04-0.66)$ & .55 \\
\hline Mechanical ventilator use & $0.41(0.07-0.73)$ & $0.58(0.19-0.82)$ & $<.001$ \\
\hline Central venous catheter use & $0.67(0.15-0.98)$ & $0.78(0.33-0.98)$ & .04 \\
\hline Indwelling urinary catheter use & $0.62(0.22-0.95)$ & $0.76(0.32-0.98)$ & $<.01$ \\
\hline Tidal volume $\leq 8 \mathrm{ml} / \mathrm{kg}$ of predicted body weight & $0.59(0.33-0.86)$ & $0.57(0.22-0.82)$ & .77 \\
\hline \multicolumn{4}{|l|}{ Clinical results } \\
\hline $\begin{array}{l}\text { Ventilator-associated pneumonia (VAP), events/1000 } \\
\text { patient-days of mechanical ventilator use, median (IQR) }\end{array}$ & $3.11(0.00-11.2)$ & $3.56(0.00-14.0)$ & .33 \\
\hline $\begin{array}{l}\text { Central-line associated bloodstream infection (CLABSI), } \\
\text { events/1000 patient-days of central venous catheter use, } \\
\text { median (IQR) }\end{array}$ & $4.95(0.00-22.0)$ & $6.29(0.00-25.6)$ & .04 \\
\hline $\begin{array}{l}\text { UTI, events/1000 patient-days of indwelling urinary catheter } \\
\text { use, median (IQR) }\end{array}$ & $6.93(0.00-19.2)$ & $8.47(0.00-36.8)$ & .37 \\
\hline Number of days on mechanical ventilation, median (IQR) & $2.48(0.28-7.07)$ & $4.28(1.39-8.43)$ & $<.001$ \\
\hline $\begin{array}{l}\text { Average ventilator-free days in a 28-day period, median } \\
\text { (IQR) }\end{array}$ & $3.25(0.47-9.70)$ & $5.38(1.54-11.10)$ & $<.001$ \\
\hline
\end{tabular}

A total of 10 characteristics were selected for the multivariable analysis. We verified that the reported practices of infection incidence density (VAP, CLABSI, and UTI) surveillance and monitoring of invasive devices utilization rate (MV and 
CVC) presented a high degree of collinearity (Table 3.3). A moderate association is also observed among the mentioned practices and the routine of registering adverse events as well as the research on infections related to invasive devices.

Table 3.3 - Cramer's V values for the association between the characteristics of structure and process

Matrix with Cramer's $V$ value for each pairwise association between the characteristics of stricture and process. Cramer's $V$ ranges from [0, 1], in which 0 indicates no association, and 1 shows a complete association. Values greater than 0.60 were considered a strong association, and those greater than 0.20 were considered a moderate association (KOTRLIK; WILLIAMS, 2003).

\begin{tabular}{|c|c|c|c|c|c|c|c|c|c|c|c|}
\hline & Structure and process & 1 & 2 & 3 & 4 & 5 & 6 & 7 & 8 & 9 & 10 \\
\hline 1 & $\begin{array}{l}\text { Exclusive routine physician per } 10 \text { beds } \\
\text { or fraction during every shift }\end{array}$ & - & & & & & & & & & \\
\hline 2 & $\begin{array}{l}\text { Transportation of patient with adequate } \\
\text { equipment }\end{array}$ & 0.01 & - & & & & & & & & \\
\hline 3 & Adverse events registering routine & 0.18 & 0.13 & - & & & & & & & \\
\hline 4 & $\begin{array}{l}\text { Research on infections related to } \\
\text { invasive devices and multiresistance for } \\
\text { clinical epidemiology }\end{array}$ & 0.06 & 0.10 & 0.11 & - & & & & & & \\
\hline 5 & 24-hour readmission rate & 0.13 & 0.03 & $\underline{0.31}$ & 0.06 & - & & & & & \\
\hline 6 & $\begin{array}{l}\text { Ventilator-associated pneumonia (VAP) } \\
\text { incidence density }\end{array}$ & 0.11 & 0.10 & $\underline{0.24}$ & $\underline{0.25}$ & $\underline{0.37}$ & - & & & & \\
\hline 7 & $\begin{array}{l}\text { Mechanical ventilation (MV) utilization } \\
\text { rate }\end{array}$ & 0.04 & 0.16 & $\underline{0.3}$ & $\underline{0.28}$ & $\underline{0.44}$ & $\underline{0.63}$ & - & & & \\
\hline 8 & $\begin{array}{l}\text { Central-line associated bloodstream } \\
\text { infection (CLABSI) incidence density }\end{array}$ & 0.11 & 0.07 & $\underline{0.32}$ & $\underline{0.36}$ & $\underline{0.34}$ & $\underline{0.80}$ & $\underline{0.59}$ & - & & \\
\hline 9 & $\begin{array}{l}\text { Central venous catheter (CVC) utilization } \\
\text { rate }\end{array}$ & 0.04 & 0.10 & $\underline{0.32}$ & $\underline{0.27}$ & $\underline{0.38}$ & $\underline{0.62}$ & $\underline{0.77}$ & $\underline{0.62}$ & - & \\
\hline 10 & $\begin{array}{l}\text { Urinary tract infection (UTI) incidence } \\
\text { density }\end{array}$ & 0.12 & 0.16 & $\underline{0.22}$ & $\underline{0.31}$ & $\underline{0.34}$ & $\underline{0.79}$ & $\underline{0.66}$ & $\underline{0.79}$ & $\underline{0.73}$ & - \\
\hline
\end{tabular}

Underscored: Coefficients greater than 0.20 were considered moderate associations.

Bold: Coefficients greater than 0.60 were considered strong associations.

IQR: Interquartile Range ( $1^{\text {st }}$ Quartile $-3^{\text {rd }}$ Quartile)

We then performed the multivariable logistic regression with adaptive elasticnet regularization (Table 3.4). The reported practices of surveillance of the central venous catheter utilization rate $(\mathrm{OR}, 1.94 ; 95 \% \mathrm{CI}, 1.32-2.94)$ and ventilatorassociated pneumonia incidence density (OR, 1.72; 95\% CI, 1.16-2.57) were independently associated with efficiency. We also observed that the effect of those variables was present in all resamples (Proportion of non-shrunk coefficients = $100 \%$ ). Some variables that presented an OR closer or equal to 1.00 (no effect), with a narrow confidence interval, which means that in some resamples, their effect 
was often or not relevant to the response variable (95\% CI, 1.00 to $1.00,0 \%$ proportion of non-shrunk coefficients) during the AENET procedure.

Table 3.4 - Results from the regularized multivariable logistic regression of the organizational characteristics

Proportion of positive responses and association of structure and process characteristics with the performance groups. We reported the frequency of units from each group. We also presented the results from the adaptive elastic-net applied to the multivariate logistic regression and the proportion of samples in which the effect of the variable was shrunk to 1.00 (no effect). Confidence intervals were estimated using 10,000 resamples. The reported practice of evaluating the rates of ventilator-associated pneumonia and the utilization of a central venous catheter were the variables associated with efficiency. Variables with $\mathrm{Cl}$ (1.00-1.00) were not relevant in any of the resampling subsets (For more details on the modeling procedure, see Appendix A2.5).

\begin{tabular}{|c|c|c|c|c|c|}
\hline Category & Structure and process & $\begin{array}{c}\text { Efficient } \\
\mathrm{n}=47(\%)\end{array}$ & $\begin{array}{l}\text { Non-efficient } \\
\mathrm{n}=71(\%)\end{array}$ & $\begin{array}{c}\text { AENET } \\
\text { multivariable } \\
\text { logistic regression } \\
\text { OR }(95 \% \mathrm{Cl})\end{array}$ & $\begin{array}{l}\text { \% of non- } \\
\text { shrunk } \\
\text { coefficients }\end{array}$ \\
\hline $\begin{array}{l}\text { Human } \\
\text { resources }\end{array}$ & $\begin{array}{l}\text { Exclusive routine } \\
\text { physician per } 10 \text { beds or } \\
\text { fraction during every shift }\end{array}$ & $43(91)$ & $70(99)$ & $0.22(0.05-0.96)$ & 99.3 \\
\hline $\begin{array}{l}\text { Transport of } \\
\text { patients }\end{array}$ & $\begin{array}{l}\text { Transportation of patient } \\
\text { with adequate equipment }\end{array}$ & $32(68)$ & $60(85)$ & $0.37(0.18-0.78)$ & 100 \\
\hline $\begin{array}{l}\text { Risk } \\
\text { management }\end{array}$ & $\begin{array}{l}\text { Adverse events } \\
\text { registering routine }\end{array}$ & $32(68)$ & $37(52)$ & $1.02(0.96-1.04)$ & 74.6 \\
\hline $\begin{array}{l}\text { Prevention of } \\
\text { health care } \\
\text { related } \\
\text { infections }\end{array}$ & $\begin{array}{l}\text { Research on infections } \\
\text { related to invasive } \\
\text { devices and } \\
\text { multiresistance for } \\
\text { clinical epidemiology }\end{array}$ & $46(98)$ & $64(9)$ & $1.91(0.87-4.99)$ & 98.9 \\
\hline \multirow[t]{6}{*}{$\begin{array}{l}\text { Quality-of- } \\
\text { care Metrics }\end{array}$} & 24-hour readmission rate & $28(60)$ & $30(42)$ & $1.00(0.99-1.00)$ & 20.8 \\
\hline & $\begin{array}{l}\text { Ventilator-associated } \\
\text { pneumonia (VAP) } \\
\text { incidence density }\end{array}$ & $43(91)$ & $48(68)$ & $1.72(1.16-2.57)$ & 99.9 \\
\hline & $\begin{array}{l}\text { Mechanical ventilation } \\
\text { (MV) utilization rate }\end{array}$ & $40(85)$ & $44(62)$ & $1.09(0.97-1.19)$ & 93.3 \\
\hline & $\begin{array}{l}\text { Central-line associated } \\
\text { bloodstream (CLABSI) } \\
\text { incidence density }\end{array}$ & $42(89)$ & $51(72)$ & $1.00(1.00-1.00)$ & 0 \\
\hline & $\begin{array}{l}\text { Central venous catheter } \\
\text { (CVC) utilization rate }\end{array}$ & $41(87)$ & $42(59)$ & $1.94(1.32-2.94)$ & 100 \\
\hline & $\begin{array}{l}\text { Urinary tract infection } \\
\text { (UTI) incidence density }\end{array}$ & $40(85)$ & $48(68)$ & $1.00(1.00-1.00)$ & 0 \\
\hline
\end{tabular}

IQR: Interquartile Range ( $1^{\text {st }}$ Quartile $-3^{\text {rd }}$ Quartile)

AENET: Adaptive Elastic-Net

\section{4}

Discussion

Our study presented a secondary observational analysis from the CHECKLIST-ICU, a multicenter cluster-randomized clinical trial conducted in 118 ICUs in a middle-income country (CAVALCANTI et al., 2016). We observed that 47 ICUs were classified as efficient in terms of risk-adjusted mortality and resource 
use. Reporting the active surveillance of nosocomial infection rates was associated with efficiency. Additionally, efficiency units presented lower CLABSI incidence density and utilization rate of invasive devices compared to non-efficient.

The efficient units mostly reported to surveil and assess the use of invasive devices utilization and nosocomial infection rates, especially CVC and VAP incidence density. We also found a high association among those variables with Cramer's V. Our results suggest a cultural behavior of self-evaluation within units using different quality-of-care metrics and not only a few. This conduct was likely to be present more often in efficient ICUs than non-efficient.

It is noteworthy that the prevention and control practices are not limited to the evaluation of metrics, but also include the bundle of care for specific conditions or illnesses, and both depend on the unit's culture (KLOMPAS, 2017). For instance, prevention practices of ventilator-associated pneumonia have been considered effective in decreasing the LOS, the number of ventilator days, and, thus, infection incidence (HEWSON-CONROY; ELLIOTT; BURRELL, 2010). This pattern can be extrapolated to other processes of care in the unit, such as interdisciplinary clinical rounds (ROTHEN et al., 2007), staffing levels (ZIMMERMAN; ALZOLA; VON RUEDEN, 2003), and visitation policies (SOARES et al., 2017).

The secondary outcomes analysis showed efficient units presented had lower utilization rates of invasive devices (MV, CVC, and IUC) and CLABSI incidence density compared to the non-efficient ICUs, even though their median SAPS-3 was higher (Table 3.1). Therefore, we hypothesize that the active surveillance of nosocomial infections may result in low infection rates in efficiency units.

Our results depict that efficient units have more implemented care protocols than non-efficient, although not statistically significant. The ORCHESTRA study (SOARES et al., 2015) identified that a higher number of implemented protocols was associated with lower mortality and resource use, in a different set of Brazilian ICUs. Implementation of infection prevention protocols is a priority for LMIC (VUKOJA et al., 2014), and they can provide better outcomes and performance for low-resource setting ICUs.

The stepwise regression (GARLAND, 2005; MCCLEAN et al., 2017; MERZ et al., 2008; NATHANSON et al., 2007; NISKANEN; REINIKAINEN; PETTILÄ, 2009; ROTHEN et al., 2007) can result in issues with the interpretability of the confidence intervals (HEINZE; WALLISCH; DUNKLER, 2018). To overcome 
this limitation, we used regularization methods (HEINZE; WALLISCH; DUNKLER, 2018). As far as we know, there is no bias correction or asymptotic distribution of ANET estimator, and it presents oracle property. Hence, we inferred the confidences intervals using the bootstrapping method, although estimates can be imprecise. To the best of our knowledge, this is the first application of regularization methods to evaluate the associations in ICU benchmarking studies. We provide a thorough discussion of the AENET model in Appendix A1.5.

We used the efficiency matrix (RAPOPORT et al., 1994; ROTHEN et al., 2007) to classify low-settings ICUs into efficient and non-efficient. Although this is a common approach for benchmarking, it has some limitations. First, the estimates of SMR and SRU may depend on the severity score applied. In this study, we used SAPS-3 as it showed proper calibration and discrimination in the Brazilian ICU population (MORALEZ et al., 2017; SILVA JUNIOR et al., 2010). Second, using the LOS as a surrogate variable for resource use is debatable since it may be influenced by the unit's process and the patient's case-mix. However, it is a reasonable proxy for measuring resource use (NEWGARD et al., 2010) and widely available, even in low-resource settings. Third, other outcomes and process indicators could be considered when defining efficiency. We evaluated mortality and resource, which are widely used metrics for assessing the performance in ICUs, both at the unit and patient-level (NOUIRA et al., 2018; SOARES et al., 2015, 2017). Nonetheless, we also considered an extensive list of structure and process characteristics to evaluate those outcome indicators. Finally, the SMR and SRU estimates could be imprecise for some ICUs, thus impacting their classification. Additionally, aggregating "underachieving," "overachieving," and "least efficient" units into a single group may hide the heterogeneities among them. However, we also observed a high linear correlation between SMR and SRU, which suggests that the dichotomized classification is valid.

\section{5}

\section{Conclusions}

By evaluating the performance groups of ICUs in low-resource settings, we found that efficient units are more likely to conduct surveillance of nosocomial infection rates compared to non-efficient units. Therefore, our results suggest that 
the management and assessment of infection control should be promptly implemented in quality improvement programs of low-resource settings ICUs. 


\section{4 \\ Article 2 - Comparing continuous versus categorical measures to assess and benchmark intensive care unit performance and efficiency}

This article is under revision by its authors and will be submitted

Purpose: To compare the use of categorical and continuous approaches combining Standardized Mortality Ratio (SMR) and Standardized Resource Use (SRU) for benchmarking performance of ICUs.

Materials and Methods: We analysed adult ICU patients, admitted between 2016 and 2018, in Brazil and The Netherlands. Performance was defined as a combination of SMR and SRU using recalibrated SAPS-3 or APACHE-IV for Brazilian or Dutch ICUs, respectively. Categorical combination was the Rapoport-Teres matrix, whereas the continuous approach was the average between the two metrics (Average Standardised Ratio, ASER). For each country, we evaluated the association among metrics using Spearman's rho coefficient and the $\mathrm{R}^{2}$ from linear regression. An expert focus group consisting of methodologists and intensivists listed potential advantages, limitations and interpretations of both SMR and SRU combinations.

Results: We included 282,303 Brazilian and 164,399 Dutch ICU admissions. Median ASER was $0.99(0.83,1.20)$ and $0.99(0.92,1.09)$ in the Brazilian and Dutch dataset respectively. Correlation between SMR and SRU with ASER in the Brazilian dataset was high ( $\mathrm{R}^{2}: 0.75$ and 0.74 for SMR and SRU, respectively), while lower in the Dutch sample $\left(\mathrm{R}^{2}: 0.67\right.$ and 0.60$)$. Continuous combination offers appropriate statistical properties for evaluating performance, especially when doing benchmarking analysis, whereas the categorical combination facilitates the interpretation but should be used with caution.

Conclusions: A continuous approach is more favourable for conducting benchmarking analysis, since it keeps desired statistical properties, especially when outcome metrics are highly correlated. The categorical approach should be used with caution as the number of ICUs is often limited. 


\section{1}

\section{Introduction}

Benchmarking assists healthcare professionals and policymakers to identify outliers and targets for quality improvement (SALLUH et al., 2018). In intensive care, benchmarking of performance is frequently applied using risk-adjusted mortality and resource use measures (WOODHOUSE et al., 2009). Intensive care unit (ICU) performance should be evaluated in different perspectives (DONABEDIAN, 1988; GARLAND, 2005; SALLUH; SOARES; KEEGAN, 2017), and standardised outcome measures have been preferred since they are case-mix adjusted and easy to interpret. The two most commonly used metrics to assess ICU performance are the standardised mortality ratio (SMR) and the standardised length-of-stay (SLOS) also called standardised resource use (SRU), which measure the clinical efficacy and the efficiency of a unit respectively (KEEGAN; GAJIC; AFESSA, 2011; NATHANSON et al., 2007; SALLUH; SOARES; KEEGAN, 2017).

Quantifying ICU performance based on a combination of these two measures is challenging. A few studies have considered two approaches to combine SMR and SRU, i.e. a categorical approach and a continuous approach. The first approach has been used more traditionally since the '90s and consists of using the Rapoport-Teres graph or "efficiency matrix" (BASTOS et al., 2020b; NATHANSON et al., 2007; RAPOPORT et al., 1994; ROTHEN et al., 2007; SOARES et al., 2015) to categorize ICUs into efficiency groups, using quadrants based on median or mean values, resulting in the categories most efficient, least efficient, overarching and underachieving. The second approach was proposed more recently and used a mathematical procedure to combine the metrics and keep the continuous nature, such as the average of the SMR and SRU for each unit, and thus obtaining a single performance metric (WORTEL et al., 2021).

Each approach has its benefits and drawbacks. The categorical approach can identify groups of interest straightforwardly, such as the best and worst-performing units (ROTHEN et al., 2007; ROTHEN; TAKALA, 2008). However, categorising a continuous outcome may result in loss of information for further inference analysis. Otherwise, the continuous approach is an attractive alternative to reduce information loss and improve the comparisons when benchmarking. However, the resulting single performance metric has yet to be assessed. (VERBURG et al., 2018). 
This study aimed to compare the categorical and the continuous approaches combining SMR and SRU to benchmark ICU performance. We hypothesize that clinicians have different opinions on the application possibilities of the two approaches and that different relations between SMR and SRU might provide different results and interpretations in practice. An ICU might be considered efficient using one approach but not when using the other, or the ICU efficiency could be affected by the data sample. Hence, we used two sets of ICUs, one from Brazil and the other from The Netherlands. We assessed the use of the average SMR and SRU in the two different settings and provided recommendations on the usage of both approaches on combining SMR and SRU.

\section{2 \\ Materials and Methods}

\subsection{1}

\section{Study design and data source}

We performed a retrospective observational analysis on data from ICU national registries in Brazil and the Netherlands (SOARES et al., 2015; VAN DE KLUNDERT et al., 2015; ZAMPIERI et al., 2019). Brazilian data was obtained from the "Organisational CHaractEriSTics in cRitical cAre" (ORCHESTRA) network (SOARES et al., 2015; ZAMPIERI et al., 2019). This dataset contains demographic and clinical data, and outcomes for adult patients ( $>=16$ years old) admitted to 134 intensive care units in 79 hospitals from 2016 to 2018 in Brazil. Patient data was retrieved from the Epimed Monitor System ${ }^{\circledR}$ (Epimed Solutions ${ }^{\circledR}$, Rio de Janeiro, Brazil) (ZAMPIERI et al., 2017). Dutch patient admission data was obtained from the Dutch National Intensive Care Evaluation (NICE) registry, a non-profit foundation established by intensivists in 1996 (VAN DE KLUNDERT et al., 2015) to evaluate ICU performance and quality of care. It consists of demographic, physiological, and clinical data and outcome of ICU patients from all Dutch ICUs in the Netherlands, mainly extracted from electronic patient records and manually validated according to stringent data quality measures. Brazilian Local Ethics Committees and the Brazilian National Ethics Committee (Brazil CAAE: 19687113.8.1001.5249) approved the study and waived the need for informed consent as it contains anonymised data. The Medical Ethics Review Committee of the Amsterdam 
University Medical Centers also waived the need for informed consent (reference number W20_192\#20.223).

\subsection{2 \\ Study population}

We included all adult ICU patients (age $>=18$ years old) in both datasets, admitted between 2016 and 2018. As both countries use different severity of illness scoring systems, in the Brazilian dataset patients were excluded based on the Simplified Acute Physiology Score 3 (SAPS-3) exclusion criteria (missing core data such as age, location before ICU admission and main ICU admission diagnosis (METNITZ et al., 2005), while in the Dutch dataset patients were excluded based on the Acute Physiology And Chronic Health Evaluation (APACHE) IV exclusion criteria. These criteria consisted of patients with an ICU length-of-stay (LOS) shorter than four hours or longer than one year; readmissions; patients admitted from another Coronary Care Unit (CCU) or ICU; patients with missing admission diagnosis or admission type; patients with burns; transplant patients or CCU or recovery patients (ZIMMERMAN et al., 2006). In addition, patients with missing SAPS-3 or APACHE-IV scores were excluded. Patient-level data furthermore consisted of the patient's demographics (age and gender), type of admission (i.e., medical, elective surgical, or urgent surgical), the severity of illness at ICU admission, i.e., the SAPS-3 used in Brazilian ICUs, and the APACHE-IV used in Dutch ICUs, the in-hospital and ICU mortality, and ICU length-of-stay in days (defined as 24hour periods based on admission and discharge dates and time). Organizational level data consisted of ICU and hospital size expressed in the number of beds.

\subsection{3}

\section{Outcomes and ICU performance}

Our primary outcome of interest was the ICU performance based on a combination of two outcome measures, the SMR and SRU, both adjusted for the severity of illness score as used in each country. The SMR corresponds to the ratio of observed number of deaths to the expected number of deaths. The expected number of deaths was obtained by the sum of mortality probabilities obtained from the recalibrated SAPS-3 standard equation (MORENO et al., 2005) or APACHE IV risk models (ZIMMERMAN et al., 2006) (Appendix A2.1). Similarly, SRU corresponds to the observed resource use to the expected resource use ratio. For this purpose, we considered the ICU LOS as a surrogate measure of ICU resource use as previously published (ROTHEN et al., 2007). We 
calculated the expected LOS with the average LOS for each decile of the recalibrated probabilities obtained from SAPS-3 (Brazil) or APACHE-IV (The Netherlands) models (Appendix A4.2).

We measured overall ICU performance in two ways: categorical approach and continuous approach. In the first, we grouped the ICUs using the SMR and SRU efficiency matrix (RAPOPORT et al., 1994; ROTHEN et al., 2007).Using the respective medians of SMR and SRU distribution, we defined the groups as follows: the most efficient (both SMR and SRU < median), underachieving (SMR >= median and SRU <= median), overachieving (SMR $<=$ median and SRU >= median), and the least efficient (both SMR and SRU > medians). Second, we used the average of SMR and SRU to obtain a single performance metric (WORTEL et al., 2021), defined as (SMR + SRU) / 2. We referred to this metric as the Average Standardised Efficiency Ratio (ASER) and the interpretation is very straightforward, the lower the ASER the better the ICU performance and efficiency than expected.

\subsection{4 \\ Statistical analysis}

We described the study population (patients and ICUs) from both countries. For continuous variables we used median and interquartile range (IQR) or mean and standard deviation (SD) depending on their distribution. For categorical variables, we used absolute frequencies and proportions.

For each country, we analysed the distribution of SMR and SRU values. We evaluated the SAPS-3 and APACHE-IV risk probabilities using the calibration belts (FINAZZI et al., 2011) (Appendix A2.1 and A2.2). We visualised the association between SMR and SRU using the efficiency matrix and estimated their correlation using Spearman's rho coefficient (VERBURG et al., 2018).

We calculated the ASER per ICU per country. To assess the ASER in each sample, we evaluated its distribution and association with SMR and SRU both combined and individually. First, we added the ASER in the efficiency matrix and observed the pattern of low and high performing units and described the distribution of ASER per efficiency group. Then, using a linear regression model, we estimated the $\mathrm{R}^{2}$ coefficient of determination to obtain the level of association using ASER as the response variable and SMR or SRU as the predictor. We also assessed potential unexpected behaviour of units due under and overestimation in ASER using funnel plots. Funnel plots are widely used 
to compare performance among units regarding one metric and identify those values outside statistical control limits (SPIEGELHALTER, 2005; VERBURG et al., 2018).

Since the severity of illness of patients in ICUs from both countries is not comparable due to the different risk scores (SAPS-3 vs APACHE-IV) used, we performed all analyses for each country separately. A p-value of 0.05 was considered significant in statistical tests, and 95\% confidence intervals were calculated using 5,000 bootstrapped samples. We performed all statistical analysis in $\mathrm{R}$ version 4.0.5.

\subsection{5}

\section{Advantages and disadvantages of SMR and SRU combinations}

In order to obtain a list of recommendations, including limitations, clinical and statistical interpretation, and implications for further use for benchmarking purposes, we conducted an expert focus group. This group consisted of four intensivists and four statisticians/methodologists and discussed and identified the main clinical and statistical advantages and disadvantages using both approaches.

\section{3 \\ Results}

Of 446,702 patients, 282,303 (63\%) were admitted to 134 Brazilian ICUs and 164,399 (37\%) were admitted to 83 Dutch ICUs. Brazilian ICUs were, on average, larger than Dutch ICUs (Table 4.1). The median age in Brazilian ICUs (Table 4.2) was comparable to the median age in Dutch ICUs, but the proportion of patients over 60 years old was higher in the Dutch population (62.9\% vs 58.7\%). Median SAPS-3 score in Brazilian patients was 43 (IQR: 24-54) and median APACHE-IV score in Dutch patients was 53 (38-75). Dutch ICUs admitted more male than female patients, this difference was lower in Brazil (male: $57.6 \%$ vs $49.0 \%$ ). Most of the ICU admissions were medical in both samples (over 60\%). Proportions of crude in-hospital and ICU mortality in Brazilian and Dutch units were comparable. ICU length of stay between Brazilian and Dutch patients was also similar (median ICU LOS 2 days IQR $(1,5)$ and 2 days IQR $(2,4)$ respectively). 
Table 4.1 - Characteristics and outcome metrics of Brazilian and Dutch ICUs

\begin{tabular}{lcc}
\hline Characteristics and outcomes & Brazil & $\begin{array}{c}\text { The } \\
\text { Netherlands }\end{array}$ \\
\hline Total number of ICUs & 134 & 83 \\
\hline ICU Beds, median (IQR) & $13(10,20)$ & $12(7,16)$ \\
Hospital Beds, median (IQR) & $214(153,368)$ & $438(315,626)$ \\
Proportion of ICU bed/Hospital bed (\%), median (IQR) & $5.6(3.3,12)$ & $2.7(2.1,3.5)$ \\
Overall outcome performance, median (IQR) & & \\
Standardized Mortality Ratio (SMR) & $0.97(0.75,1.21)$ & $1.00(0.89,1.12)$ \\
Standardized Resource Use (SRU) & $1.06(0.79,1.34)$ & $0.98(0.88,1.08)$ \\
Averaged Standardized Efficiency Ratio (ASER) & $0.98(0.81,1.23)$ & $0.99(0.92,1.09)$ \\
\hline
\end{tabular}

ICU: Intensive Care Unit

IQR: Interquartile Range (1st Quartile, 3rd Quartile)

ASER: Average between SMR and SRU

(A) Brazil

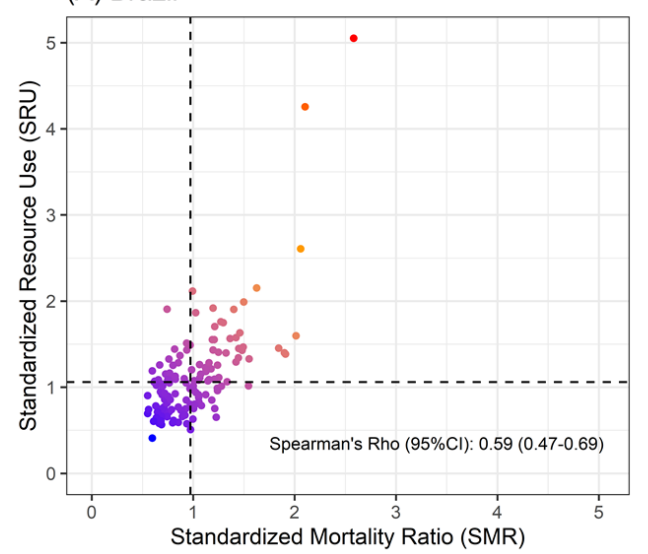

(B) The Netherlands

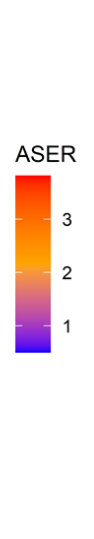

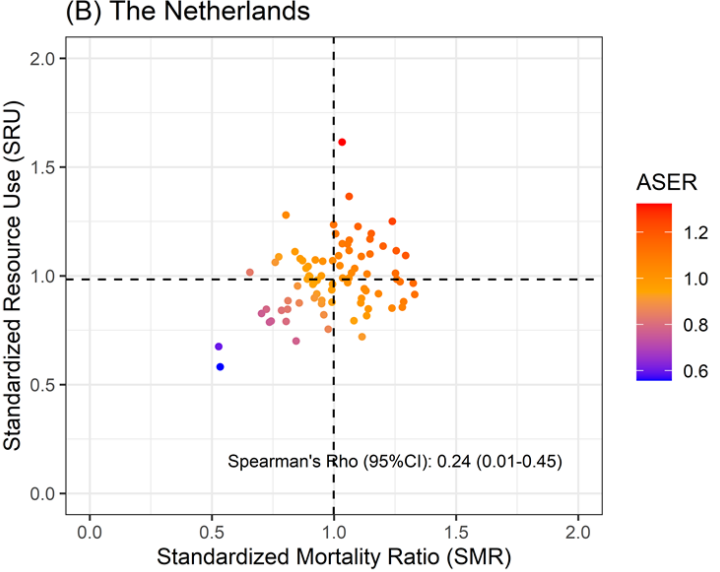

Figure 4.1 Distribution of SMR, SRU, and ASER values in the efficiency matrix in (A) Brazilian ICUs and (B) Dutch ICUs

The distribution of SMR and SRU was quite different between the two countries (Figure 4.1, Appendix A2.3 and A2.4). Brazilian units showed larger variability in SMR and SRU compared to Dutch units (Table 4.1, Figure 4.1). The SMR and SRU were more correlated in Brazilian ICUs than in Dutch ICUs (Spearman's Rho: 0.59 vs 0.24 ). The proportion of overachieving or underachieving units, taken as one group, was lower in Brazil (30\% vs 41\%, Appendix A4.3). When observing the ASER values, for both countries, the highest and lowest values of ASER were concentrated in the least and most efficient groups, respectively. However, Brazil has more units with high SMR or SRU, which was expressed as a larger number of extreme ASER values, mostly concentrated in the "least efficient" group. In contrast, Dutch units are more concentrated toward the median (Figure 4.1). 
Table 4.2 - Characteristics and outcomes of critically ill patients in Brazil and The Netherlands

\begin{tabular}{|c|c|c|}
\hline Characteristics and Outcomes & Brazil & $\begin{array}{c}\text { The } \\
\text { Netherlands }\end{array}$ \\
\hline Total number of patients & 282,303 & 164,399 \\
\hline Age (years), median (IQR) & $65(49,78)$ & $66(54,75)$ \\
\hline $16-30, N(\%)$ & $19,877(7.0)$ & $9,599(5.8)$ \\
\hline $31-40$ & $26,214(9.3)$ & $8,558(5.2)$ \\
\hline $41-50$ & $28,591(10)$ & $15,300(9.3)$ \\
\hline $51-60$ & $41,878(15)$ & 27,707 (16.9) \\
\hline $61-70$ & $53,435(19)$ & $42,542(25.9)$ \\
\hline $71-80$ & $53,956(19)$ & $41,572(25.3)$ \\
\hline $81-90$ & $45,737(16.2)$ & $17,716(10.8)$ \\
\hline$>90$ & $12,615(4.5)$ & $1,405(0.9)$ \\
\hline \multicolumn{3}{|l|}{ Gender, N (\%) } \\
\hline Female & $142,848(51)$ & $69,636(42.4)$ \\
\hline Male & $139,314(49)$ & $94,748(57.6)$ \\
\hline Unknown/Transgender & $141(<0.1)$ & $15(<0.1)$ \\
\hline \multicolumn{3}{|l|}{ Admission type, $\mathbf{N}(\%)$} \\
\hline Medical & $186,235(66)$ & $100,252(61.0)$ \\
\hline Elective surgery & $75,832(27)$ & $43,742(26.6)$ \\
\hline Urgent surgery & $20,236(7.2)$ & $20,405(12.4)$ \\
\hline \multicolumn{3}{|l|}{ Severity-of-illness score, median (IQR) } \\
\hline $\begin{array}{l}\text { Simplified Acute Physiology Score (SAPS-3) } \\
\text { Acute Physiology and Chronic Health Evaluation }\end{array}$ & $43(34,54)$ & - \\
\hline $\begin{array}{l}\text { (APACHE IV) } \\
\text { Predicted mortality risk }\end{array}$ & - & $53(38,75)$ \\
\hline SAPS-3 Predicted mortality risk & $\begin{array}{l}0.09(0.03 \\
0.24)\end{array}$ & - \\
\hline APACHE-IV Predicted mortality risk & 2 & $\begin{array}{l}0.08(0.03 \\
0.26)\end{array}$ \\
\hline \multicolumn{3}{|l|}{ Outcomes } \\
\hline ICU Length-of-Stay (days), median (IQR) & $2(1,5)$ & $2(2,4)$ \\
\hline ICU mortality, $\mathrm{N}(\%)$ & $25,510(9.0)$ & $16,048(9.8)$ \\
\hline Hospital mortality, N (\%) & $38,898(13.7)$ & $23,023(14.0)$ \\
\hline
\end{tabular}

Figure 4.2 shows the association between SMR and SRU with the ASER using a linear regression model. In the Brazilian sample, the association between SMR and SRU with ASER was high (R2: 0.77 and 0.91 for SMR and SRU, respectively), while in the Dutch sample, this association was lower $\left(\mathrm{R}^{2}\right.$ : 0.67 and 0.60 for SMR and SRU respectively). We used a funnel plot to assess under or overestimation of ASER values among units. In both datasets, the ICUs were mostly located within the $95 \%$ and $99.8 \%$ control limits for SMR, SRU and ASER, and there were no signs of under or overestimation of values. 
(A) Brazil

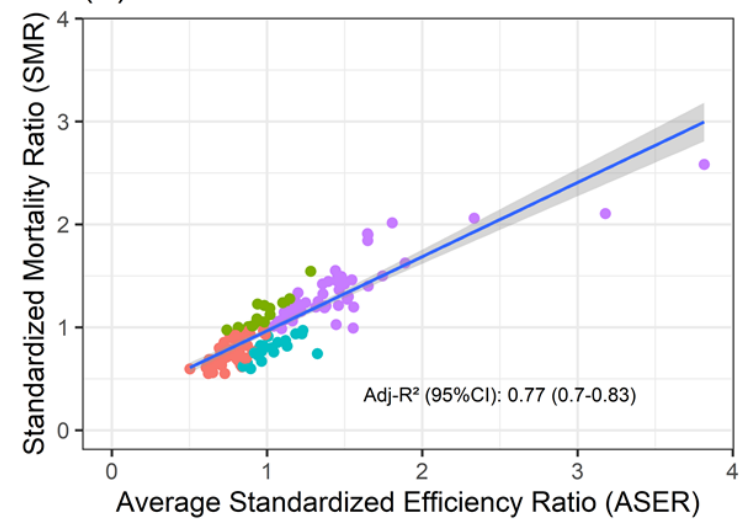

(B) The Netherlands

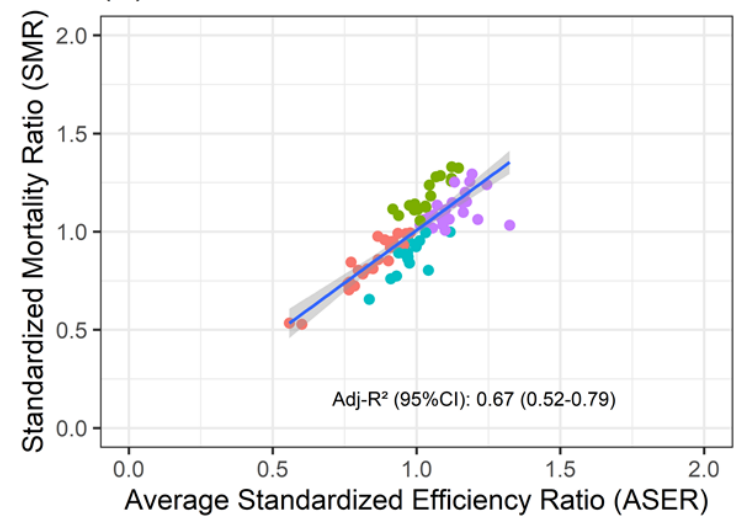

Efficiency Group • Most Efficient $\quad$ Underachievin
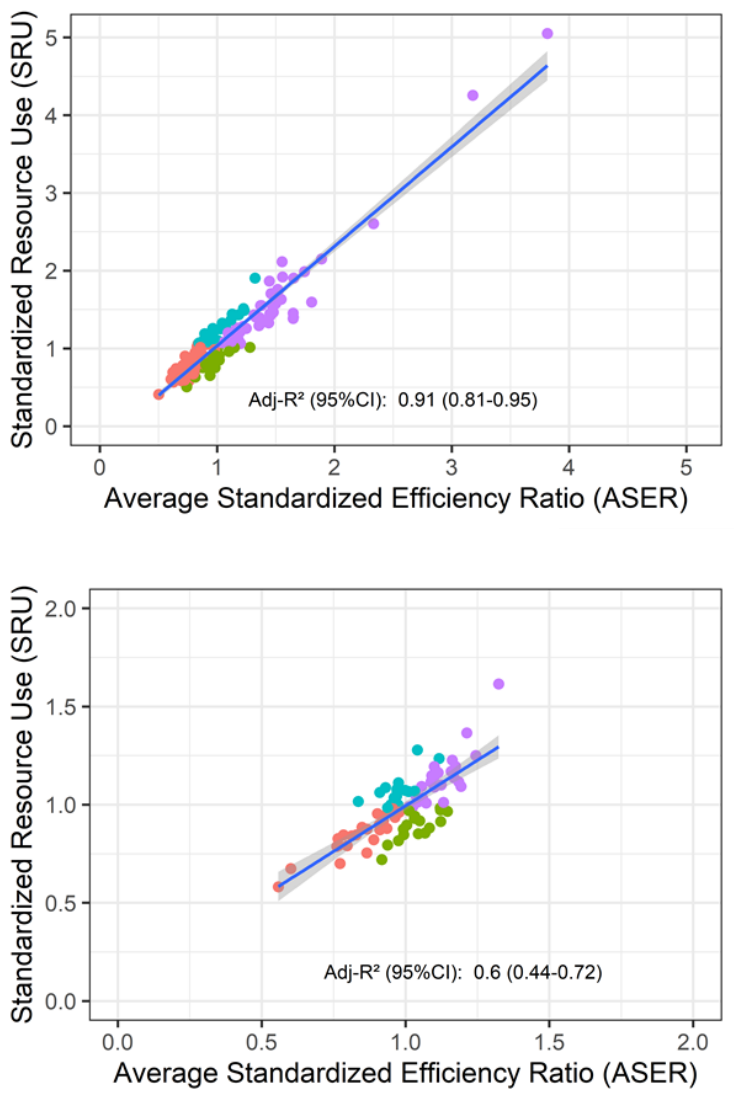

Figure 4.2 - Association between SMR and SRU with ASER in (A) Brazilian ICUs and (B) Dutch ICUs. Bisector line represents a perfect correlation between metrics. The blue line is the regression line with confidence intervals (shaded area). $R^{2}$ was obtained from the regression line using the ASER as a predictor for SMR or SRU. 
(A) Brazil

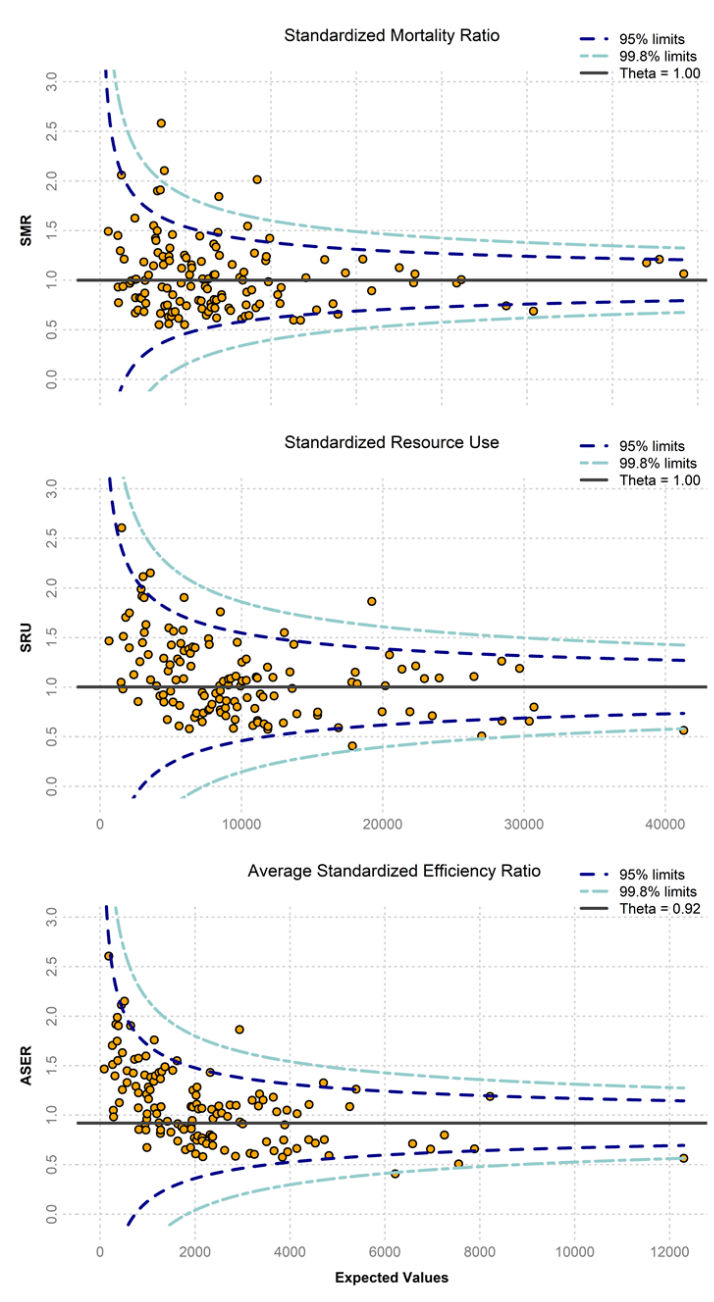

(B) The Netherlands
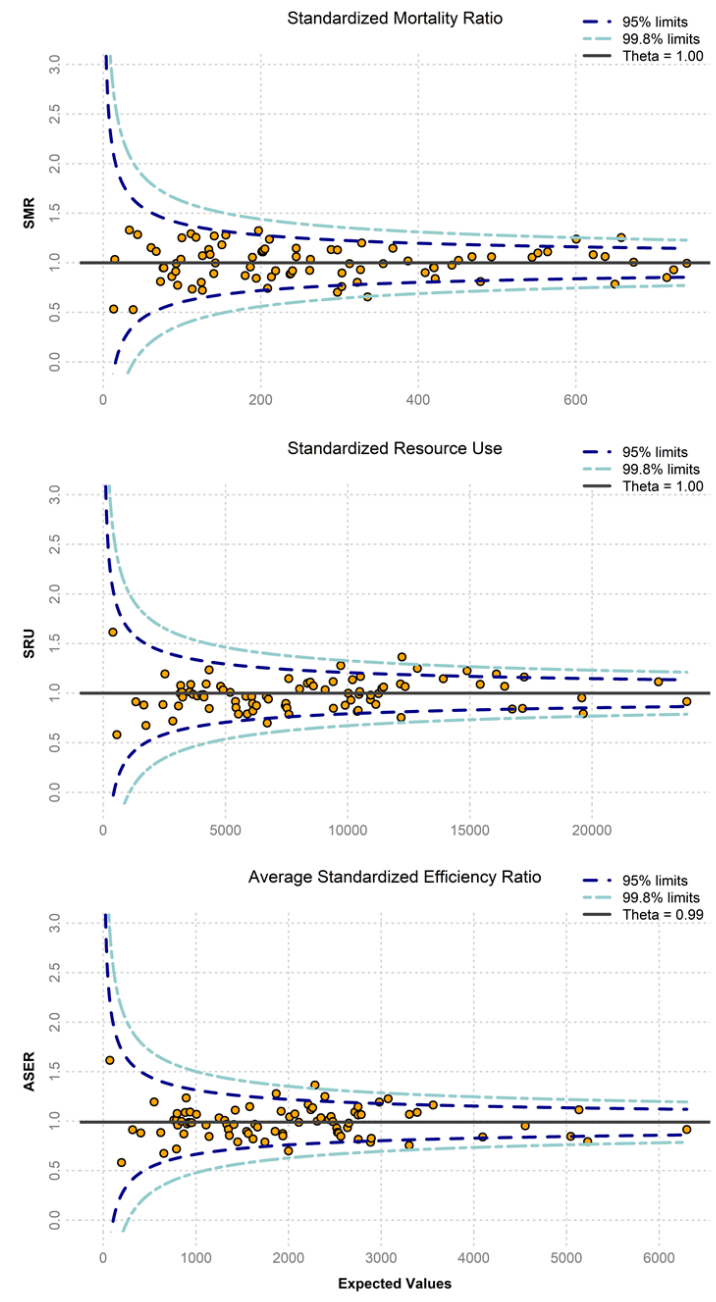

Figure 4.3 - Funnel plots for SMR, SRU and ASER in (A) Brazilian ICUs and (B) Dutch ICUs. Dashed lines represent control limits: dark blue - 95\%; and light blue - 99.8\%. Theta is the baseline value for each metric obtained as the observed/expected value.

Inspired by these quantitative results, the expert focus group composed a list of potential advantages, limitations and statistical and clinical interpretations of considering a categorical and continuous approach for combining SMR and SRU (Table 4.3). Mainly, when using the categorical approach, ICUs can be classified into groups and further subgroup analyses can be performed on the groups of interest, while when using the continuous approach ICUs are not necessarily similar in terms of performance: there is a range of values of performance metrics that can be analysed. In contrast, the categorical approach consider ICUs closely but at the other side of the categorical boundaries (very) different while they perform similar. Additionally, when using the categorical approach, a larger data sample is needed to obtain consistent estimates for further statistical analysis, while when using the continuous approach, a large sample is not necessarily needed. 
Table 4.3 - Advantages and disadvantages of using a categorical/dichotomous versus the continuous representation of ICU

\begin{tabular}{|c|c|c|}
\hline & Dichotomous/Categorical & Continuous \\
\hline Definition & $\begin{array}{l}\text { Classify ICUs into groups of efficiency } \\
\text { (“Efficiency matrix", Rapport et al, } \\
\text { 1994/Rothen et al, 2007): } \\
\text { Most efficient ICUs are defined as } \\
\text { ICUs with an SMR < median SMR and } \\
\text { SRU < median SRU, least efficient } \\
\text { ICUs are defined as SMR > median } \\
\text { SMR and SRU > median SRU }\end{array}$ & $\begin{array}{l}\text { Calculate the arithmetic mean between } \\
\text { SMR and SRU. } \\
\text { Averaged Standardized Efficiency } \\
\text { Ratio (ASER) } \\
\text { ASER = (SMR + SRU) } / 2\end{array}$ \\
\hline Studies where the outcome is used & $\begin{array}{l}\text { [Rothen et al. 2007] } \\
\text { [Nathason et al 2007] } \\
\text { [Soares et al. 2015] } \\
\text { [Bastos et al. 2020] }\end{array}$ & [Wortel et al. 2021] \\
\hline Statistical analyses & $\begin{array}{l}\text { Simplifies statistical analyses. } \\
\text { Units in the same group are assumed } \\
\text { to have similar performance. } \\
\text { Can evaluate differences among } \\
\text { groups of efficiency using statistical } \\
\text { tests or regression analysis (e.g: } \\
\text { comparing most efficient vs least } \\
\text { efficient units) } \\
\text { Subgroups of ICUs can be further } \\
\text { explored }\end{array}$ & $\begin{array}{l}\text { Fewer observations/ICUs needed to } \\
\text { observe effects. } \\
\text { Units are not necessarily similar in } \\
\text { terms of performance: there is a span } \\
\text { of values of performance metrics that } \\
\text { can be analysed. } \\
\text { Depending on the distribution of the } \\
\text { ASER, multiple parametric or non- } \\
\text { parametric statistical analyses can } \\
\text { straightforwardly be applied. }\end{array}$ \\
\hline Limitations & $\begin{array}{l}\text { A larger number of observations/ICUs } \\
\text { needed to obtain consistent estimates. } \\
\text { Dichotomisation would lead to a loss of } \\
\text { information about the true relationship } \\
\text { between variables, which often } \\
\text { translates into a loss of statistical } \\
\text { power and a decreased effect size. } \\
\text { Choosing two groups (e.g., most } \\
\text { efficient vs least efficient units) may } \\
\text { limit the power of the analysis. } \\
\text { When regression is being used to } \\
\text { adjust for the effect of a confounding } \\
\text { variable, dichotomisation will run the } \\
\text { risk that a substantial part of the } \\
\text { confounding remains. }\end{array}$ & $\begin{array}{l}\text { Averaging SMR and SRU may not be } \\
\text { fully representative of a unit's actual } \\
\text { performance (E.g., units with high SMR } \\
\text { and low SMR may present an average } \\
\text { closer to the reference line) } \\
\text { SMR and SRU may have different } \\
\text { weights during the decision-making } \\
\text { process. }\end{array}$ \\
\hline Statistical interpretation & $\begin{array}{l}\text { Generally, there is no good reason to } \\
\text { suppose that there is an underlying } \\
\text { dichotomy, and if one exists, there is } \\
\text { no reason it should be at the median. } \\
\text { Therefore, interpretation of the results } \\
\text { is highly dependent on the chosen cut- } \\
\text { off point (e.g., median SMR and } \\
\text { median SRU). } \\
\text { It makes it challenging to model other } \\
\text { categories of ICUs, e.g., under- and } \\
\text { overachieving ICUs (ICUs with SMR < } \\
\text { median SMR and SRU > median SRU } \\
\text { and vice versa) }\end{array}$ & $\begin{array}{l}\text { For ICUs with very low SMR and very } \\
\text { high SRU (or vice versa), the resulting } \\
\text { ASER is distorted. }\end{array}$ \\
\hline
\end{tabular}




\begin{tabular}{|c|c|c|}
\hline & $\begin{array}{l}\text { Dichotomisation conceals any non- } \\
\text { linearity in the relation between the } \\
\text { variable and outcome. }\end{array}$ & \\
\hline Clinical interpretation of the definition & $\begin{array}{l}\text { Interpretation of ICUs performance is } \\
\text { straightforward: you are either efficient } \\
\text { or not. } \\
\text { ICUs close to the cut-off point but on } \\
\text { opposite sides are characterised as } \\
\text { being very different rather than very } \\
\text { similar. }\end{array}$ & $\begin{array}{l}\text { Interpretation is not always clear: e.g., } \\
\text { it is difficult to identify which ICUs are } \\
\text { 'good' and 'bad', and there is no cut-off } \\
\text { point. } \\
\text { It might be more important for some } \\
\text { ICUs to know how they score on the } \\
\text { SMR, while others might find their } \\
\text { performance based on SRU more } \\
\text { important. With a single average score, } \\
\text { it is unclear which of the underlying } \\
\text { indicators the ICU could improve. }\end{array}$ \\
\hline
\end{tabular}

ASER: Averaged Standardized Efficiency Ratio; ICU: Intensive Care Unit; SMR: Standardized Mortality Ratio; SRU: Standardized Resource Use

\section{4 \\ Discussion}

We evaluated two approaches to combine SMR and SRU for ICU benchmarking using data of two large national ICU registries in Brazil and in The Netherlands. We observed that the correlation between SMR and SRU influences the properties of their combination. A high positive correlation setting between SMR and SRU favours using the average as a general efficiency metric, whereas a lower correlation provides a balanced distribution of units per quadrant in the efficiency matrix. Funnel plots showed that units had ASER values within statistical control limits with no apparent under or overestimation. In addition, we assessed the average of SMR and SRU as a combination for further benchmarking studies in settings similar to Brazilian or Dutch ICUs.

The high association between SMR and SRU with ASER in the Brazilian sample indicates that increasing ASER corresponds to increasing SMR and SRU. This association is lower in the Dutch sample than in the Brazilian sample, and the most and least efficient groups become less distinguishable from under or overachieving groups (Figure 4.2). This potentially indicates that low correlation between SMR and SRU affected the distribution of the ASER. In addition, the "cut-offs" for defining the efficiency groups may provide misclassifications and produce unreliable results, thus decreasing the rankability. We note that, although large, the Brazilian data corresponds to a convenience sample, whereas the Dutch data comes from a complete coverage national database, thus coverage is different. However, the heterogeneity in the Brazilian healthcare system we describe here has been previously evidenced(MACHADO et al., 2017; RANZANI et al., 2021) 
When evaluating more than one measure of performance, the combination is a natural approach (REEVES et al., 2007). Decisions on whether to combine or not are essential in ICU benchmarking since metrics must represent the unit's performance, and their interpretation can influence clinical and managerial decision-making. Categorising into single continuous metrics or variables have been published before (BASTOS et al., 2020b; NATHANSON et al., 2007; ROTHEN et al., 2007; SOARES et al., 2015). In our focus group we identified similar implications for using the efficiency matrix or the average SMR and SRU.

A continuous approach retains the original information and interpretation of performance among units and enables a more straightforward comparison among units. On the other hand, using the categorical/dichotomous approach provides a straightforward (clinical) interpretation (FARRINGTON; LOEBER, 2000) since an ICU is positioned into a specific performance category. However, units in different efficiency groups but very close to the cut-off points are considered to be different while their SMR and SRU values might be very similar. Furthermore, units from the same group are considered equal and, thus, this loss of information may harm further analyses, especially when the sample size is already small (ALTMAN; ROYSTON, 2006; MACCALLUM et al., 2002). since an ICU is positioned into a specific performance category. However, units in different efficiency groups but very close to the cut-off points are considered to be different while their SMR and SRU values might be very similar. Furthermore, units from the same group are considered equal and, thus, this loss of information may harm further analyses, especially when the sample size is already small (DAWSON; WEISS, 2012; NUZZO, 2019).

The choice of one of those approaches will depend on the management, research, or clinical objectives. If one aims to identify performance groups instead of individual comparison, then a categorical approach seems adequate. However, if the goal is to compare individual performance, a continuous approach may be preferable. For instance, continuous metrics may provide better statistical properties and more robust results in inference analysis, such as studies that evaluate the association between organisational factors and ICU efficiency (BASTOS et al., 2020b; ROTHEN; TAKALA, 2008; SOARES et al., 2015; WORTEL et al., 2021). This might explain potential nonsignificant results due to dichotomising performance in addition to reduced sample size when comparing two groups (e.g., "least" and "most" efficient groups). 
We evaluated the association between the correlation of SMR and SRU with their combination approaches. SMR and SRU presented a high positive correlation for Brazilian ICUs, which resulted in units more concentrated in quadrants of "least" and "most" efficient units in the efficiency matrix. For Dutch units, the correlation was lower, and all efficiency quadrants were more equally distributed (Figure 4.1). However, in both countries, ASER was correlated with SMR and SRU individually, especially for Brazilian units (Figure 4.2). Hence, the average SMR and SRU could be used as a general efficiency measure in those settings of moderate/high correlation between those metrics.

The strengths of our work consist in being an analysis of outcome metrics from large national registries of ICUs in two distinct countries, Brazil and The Netherlands. For all patients we had information on the severity of illness and outcomes. The present study also has some limitations. First, comparisons among countries were not possible due to differences in the locally adopted severity of illness score. However, we performed our analysis per country, considering their distribution of SMR and SRU. Second, our analysis was limited to the combination of two metrics. If more than two metrics present a considerable degree of colinearity, using the average may give reasonable results. Conversely, the categorisation becomes more challenging in case of multiple dimensions. Hence, different methods for combining, such as clustering or data envelopment analysis, should be applied. Our study analysed the two main metrics used in ICU benchmarking, SMR and SRU, which also measure different performance perspectives.

\section{5 \\ Conclusion}

Combining measures of quality indicators will always conceal some degree of information. We observed that a continuous approach is more favourable for conducting benchmarking analysis, since it keeps desired statistical properties, especially when outcome metrics are highly correlated. The categorical approach should be used with caution as the number of ICUs is often limited. 


\section{Article 3 - Increased number of nurses per bed is associated with higher efficiency in intensive care units: An analysis of the ORCHESTRA database}

This article is under revision by its authors and will be submitted

Purpose: Measuring the effect of organisational factors on intensive care unit (ICU) performance provides potential targets for improvement. We aimed to find the organisational factors that drive efficiency in Brazilian ICUs by comparing two modelling approaches for confounder adjustment.

Methods: A retrospective analysis of the "Organisational CHaractEriSTics in cRitical cAre" (ORCHESTRA) study, a multicenter database of Brazilian ICUs. ICU efficiency was calculated as the average of the Standardised Mortality Ratio (SMR) and the Standardised Resource Use (SRU). We estimated and compared the average treatment effect (ATE) of seven organisational factors in ICU efficiency using two modelling approaches: linear regression with propensity scores and causal random forests (CRFs).

Results: We analysed 284,250 patients admitted to 134 ICUs in 69 Brazilian hospitals. Overall median SMR was 0.95 [IQR: 0.67,1.26], median SRU was 1.03 [IQR: 0.82,1.22] and median ICU efficiency was 0.98 [IQR: 0.80,1.25]. In both linear regression model and $\mathrm{CRF}$, the average number of nurses per ten beds was independently associated with ICU efficiency (ATE [95\% CI]: -0.12 [-0.20, -0.04] and -0.09 [-0.16, -0.02], respectively). The CRF additionally found an association between the average number of physicians per ten beds and ICU efficiency, ATE [95\% CI]: 0.11 [0.01, 0.20], which diminished after removing outlying ICUs.

Conclusion: Increased nurse per bed ratio was associated with high ICU efficiency in a large sample of Brazilian ICUs. Results between models were similar, indicating a potential linear pattern of the association, but CRFs were more sensible to the data values and sample size. 


\section{1 \\ Introduction}

Benchmarking intensive care units (ICUs) provides critical care practitioners and administrators possibilities for improvement in the process of care and outcomes (GARLAND, 2005; LANGE; DONGELMANS; KEIZER, 2017; SALLUH; SOARES; KEEGAN, 2017; WOODHOUSE et al., 2009), especially in limited-resource settings (BOZZA; SALLUH, 2010; SALLUH; SOARES; KEEGAN, 2017). Doing this, it is crucial to understand how organizational patterns affect the outcomes and performance in an ICU. Risk-adjusted indicators have been used to measure the efficiency of an ICU: the standardized mortality ratio (SMR) and the standardized resource use (SRU) (BASTOS et al., 2020b; ROTHEN et al., 2007; SOARES et al., 2015; WORTEL et al., 2021).

Previous studies have evaluated the associations between several organizational factors and ICU outcomes in various ways. The presence of clinical rounds (ROTHEN et al., 2007), improved team communication strategies (CHECKLEY et al., 2014), the adherence to best practices and care protocols (SOARES et al., 2015), the combination of 24/7 expert intensivist coverage, the presence of a dedicated pharmacist, and high nursing autonomy (ZAMPIERI et al., 2019), the active surveillance of nosocomial infections (BASTOS et al., 2020b), and intensivists per ICU bed (WORTEL et al., 2021) were associated with better ICU outcomes and efficiency. Different characteristics of an ICU may influence the organizational factors that drive ICU efficiency. Hence, their confounding effects should be considered, which was not always adequately applied in previous studies.

Confounding adjustment is traditionally performed with propensity scores used as a covariate in a regression model. Recently, machine learning models such as the Bayesian Additive Regression Trees (BART) (CHIPMAN; GEORGE; MCCULLOCH, 2012) and Causal Random Forests (CRF) (WAGER; ATHEY, 2018) have been proposed. With those models, confounding adjustment is performed with a partitioning procedure of the data using decision trees, and their non-parametric nature provides the modelling of nonlinear relationships (ATHEY; TIBSHIRANI; WAGER, 2019; WAGER; ATHEY, 2018), which form advantages over the assumptions of linear regression modelling. Moreover, CRFs provide unbiased estimates of the average treatment effect (ATE) and confidence intervals compared to previous tree-based models (ATHEY; TIBSHIRANI; WAGER, 2019; WAGER; ATHEY, 2018). 
Understanding the measured effect of organisational factors on ICU performance is essential to identify targets for improvement. Our goal is to investigate the association of organisational factors with efficiency in ICUs by comparing linear regression with propensity scores and causal random forests.

\section{2 \\ Methods}

\section{2 .1}

\section{Study design and data source}

We performed a retrospective observational analysis on the "ORganizational CHaractEriSTics in cRitical cAre" (ORCHESTRA) data (SOARES et al., 2015; ZAMPIERI et al., 2017, 2019). This prospectively collected data set contains demographic and clinical information, use of resources, and outcomes of adult patients $(>=16$ years old) admitted to 134 intensive care units in 79 hospitals from 2016 to 2018 in Brazil (SOARES et al., 2015; ZAMPIERI et al., 2017, 2019). Information was retrieved from the Epimed Monitor System ${ }^{\circledR}$ (Epimed Solutions ${ }^{\circledR}$, Rio de Janeiro, Brazil) (ZAMPIERI et al., 2017). Local Ethics Committees and the Brazilian National Ethics Committee (CAAE: 19687113.8.1001.5249) approved the study and waived the need for informed consent.

\section{2 .2 \\ Study population}

We included data at the patient and the unit level. At the patient level, we considered the patient's demographics, the severity of illness at admission using the Simplified Acute Physiology Score 3 (SAPS-3), the in-hospital mortality, and ICU length-of-stay in days (defined as 24-hour periods). At the organisational level, information on hospital and ICU characteristics of structure and process was collected by a structured survey to the ICU director and/or the chief nurse that included: hospital and ICU bed capacity, the type of ICU (surgical, oncological or neurological ICU), the presence of training programs in critical care certified by the Brazilian Association of Intensive Care (Associação Brasileira de Medicina Intensiva-AMIB), and ICU staffing patterns, such as the average number of physicians per bed and the average number of nurses per bed in each daily 
shift (considering day and night), the full-time coverage of a board-certified intensivist and the annual bed ICU occupancy rate. We note that the organisational variables were provided as the most representative information from the period of analysis (2016-2018).

\section{2 .3}

\section{Outcomes}

Our primary outcome is ICU efficiency as a combination of two outcome indicators: the standardised or risk-adjusted in-hospital mortality rate and the standardised or riskadjusted resource utilisation.

The Standardised Mortality Ratio (SMR) corresponds to the ratio of the observed number of deaths to the expected number of deaths. The expected number of deaths was obtained by adding up mortality probabilities obtained from the recalibrated SAPS-3 risk model.

Similarly, the Standardised Resource Use (SRU) corresponds to the observed resource use to the expected resource use ratio. For this purpose, we considered the hospital length-of-stay (LOS) as a surrogate measure of resource use (ROTHEN et al., 2007). Expected LOS was calculated with the average LOS for each decile of the SAPS3 estimated probability from the sample.

Finally, we used the average of SMR and SRU to obtain a single efficiency measurement, referred to as Averaged Standardised Efficiency Ratio (ASER), with an interpretation similar to the previous metrics: the lower the value, the better is the performance of a particular unit.

\section{2 .4}

\section{Statistical Analysis}

We described the study population using median and interquartile range (IQR) for continuous variables and absolute frequency and proportions for categorical variables. We evaluated the relationship between SMR and SRU using Spearman's rho correlation coefficient and scatter plots. We evaluated the association of nine organizational factors with efficiency (ASER). We used scatterplots to visually assess the pattern of association of each organizational variable with the efficiency measure.

We used linear regression with propensity scores and the Causal Random Forests in multivariable analyses, a machine learning algorithm that estimates the average treatment effect (ATHEY; TIBSHIRANI; WAGER, 2019; WAGER; ATHEY, 2018). The CRF consists of a set of decision trees in which data is split into two parts: one to 
build each tree, using the classic CART algorithm and the other to estimate the treatment effects, a process called "honest splitting." Although previous tree algorithms have been proposed, the CRF has the benefits of using non-parametric modelling, which allows the capture of nonlinear relationships. Its ATE estimates are unbiased and asymptotically normal, thus providing reliable confidence intervals (ATHEY; IMBENS, 2016; WAGER; ATHEY, 2018).

For each method, the process for confounder identification followed a combined approach of using knowledge of specialists and statistical methods, such as the ChangeIn-Estimate (MALDONADO; GREENLAND, 1993; MICKEY; GREENLAND, 1989). To estimate propensity scores for the regression model, we used a logistic regression model for categorical organizational variables and the generalized propensity scores (HIRANO; IMBENS, 2004; ROSENBAUM; RUBIN, 1983) for continuous variables. The CRF does not require a previously calculated propensity score since it uses propensity trees from the covariates to estimate the propensity for the treatment variable (organizational factors).

As a sensitivity analysis, we removed units with extreme values of ASER. The presence of extremely good or worst performance units may influence our results. We identified those outlying units using the z-score method with three standard deviations. We re-estimated the mortality probabilities, the efficiency measures, and the average treatment effects for each organizational factor, using the linear model and the CRF, after removing the outlying units. Also, we used those results to evaluate the pattern of the associations identified between both models, such as non-linearities.

All analyses were performed in $\mathrm{R} 4.0 .2$, with tidyverse for data preparation and preparation, and $g r f$ (Generalized Random Forests) (ATHEY; TIBSHIRANI; WAGER, 2019) for fitting Causal Random Forests.

\section{3}

\section{Results}

We analysed 284,250 patients admitted to 134 participating ICUs in 79 hospitals. The median age of patients was 65 years [IQR: 49, 78], with 58\% being older than 60 years old, $51 \%$ were women, and most of the admissions were medical (66\%) (Table 5.1). The overall median SMR was 0.95 [IQR: 0.67, 1.26], median SRU was 1.03 [IQR:0.82, 1.22] and the median ASER was 0.98 [IQR: 0.80, 1.25] (Table 5.2). There was a mild 
correlation between the SMR and SRU (Spearman's rho correlation coefficient: 0.54 , Figure 5.1).

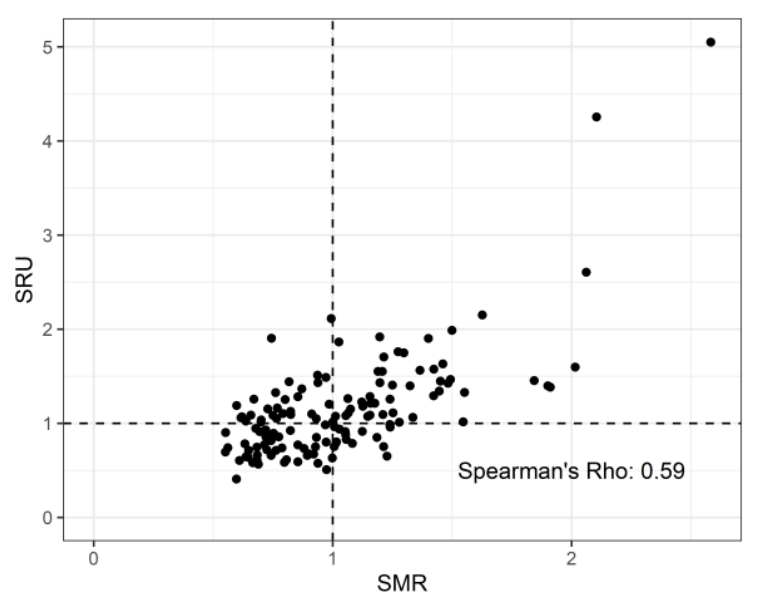

Figure 5.1 - Association between SMR and SRU with ASER in (A) Brazilian ICUs and (B) Dutch ICUs. Bisector line represents a perfect correlation between metrics. The blue line is the regression line with confidence intervals (shaded area). $\mathrm{R}^{2}$ was obtained from the regression line using the ASER as a predictor for SMR or SRU.

Table 5.1 - Characteristics and outcomes of Intensive Care Unit patients

\begin{tabular}{|l|c|}
\hline Characteristic & 284,250 \\
\hline Total number of patients & $65[49,78]$ \\
\hline Age, median [IQR] & $21,824(7.7 \%)$ \\
\hline$\leq 30, \mathrm{~N}(\%)$ & $26,214(9.2 \%)$ \\
\hline $31-40$ & $28,591(10 \%)$ \\
\hline $41-50$ & $41,878(15 \%)$ \\
\hline $51-60$ & $53,435(19 \%)$ \\
\hline $61-70$ & $53,956(19 \%)$ \\
\hline $71-80$ & $45,737(16 \%)$ \\
\hline $81-90$ & $12,615(4.4 \%)$ \\
\hline$>90$ & \\
\hline Gender, N (\%) & $143,765(51 \%)$ \\
\hline Female & $140,342(49 \%)$ \\
\hline Male & $143(<0.1 \%)$ \\
\hline Unknown/Transgender & \\
\hline Charlson Comorbidity Index [N = 280,286] & $1.57(1.91)$ \\
\hline Mean (SD) & $1[0,2]$ \\
\hline Median [IQR] & \\
\hline Admission type, N (\%) & $187,617(66 \%)$ \\
\hline Medical & $76,196(27 \%)$ \\
\hline Elective surgery & $20,437(7.2 \%)$ \\
\hline Urgent surgery & $2[1,5]$ \\
\hline ICU Length-of-Stay, median [IQR] & $25,605(9.0 \%)$ \\
\hline ICU mortality, N (\%) & $25,649(9.0 \%)$ \\
\hline Hospital mortality, N (\%) & \\
\hline
\end{tabular}

ICU: Intensive Care Unit.

IQR: Interquartile Range (1st Quartile - 3rd Quartile).

SD: Standard Deviation. 
Regarding the organisational characteristics of the included ICUs, the median number of physicians per ten beds is 1.5 [IQR: 1.36, 1.95], and the median number of nurses per ten beds is 1.79 [IQR: 1.43, 2.50]. Twenty-three ICUs (23\%) are specialised, $20 \%$ have medical residency in critical care, and less the half $(41 \%)$ are full-time covered by a certified intensivist (Table 5.2).

To measure the association of organisational factors (exposures) with efficiency (ASER), we defined the variables for confounder adjustment (Table 5.3). Exposure variable ICU to hospital bed ratio had the least number of confounders $(\mathrm{N}=2)$, while the average nurse per ten beds had the most $(\mathrm{N}=7)$. We observed an apparent pattern of negative correlation between the average nurse per ten beds with the ASER.

Table 5.2 - Outcome measures and organizational characteristics of all Intensive Care Units

\begin{tabular}{|l|c|}
\hline Characteristics and Measurements & \\
\hline Number of units & 134 \\
\hline Outcome measures, median [IQR] & \\
\hline Standardized Mortality Ratio (SMR) & $0.95[0.67,1.26]$ \\
\hline Standardized Resource Use (SRU) & $1.03[0.82,1.22]$ \\
\hline Averaged Standardized Efficiency Ratio (ASER) ${ }^{\mathrm{a}}$ & $0.98[0.80,1.25]$ \\
\hline Organizational characteristics & \\
\hline Hospital beds, median [IQR] & $214[153,368]$ \\
\hline ICU beds, median [IQR] & $13[10,20]$ \\
\hline Specialized ICUs ${ }^{\text {b }}, \mathrm{N}(\%)$ & $23(17 \%)$ \\
\hline Presence of medical residency in critical care, N (\%) & $27(20 \%)$ \\
\hline Presence of a full-time board-certified intensivist, N (\%) & $41(31 \%)$ \\
\hline Average Physicians per 10 beds ${ }^{c}$, median [IQR] & $1.50[1.36,1.95]$ \\
\hline Average Nurses per 10 beds ${ }^{c}$, median [IQR] & $1.79[1.43,2.50]$ \\
\hline Admissions per bed, median [IQR] & $128[84,169]$ \\
\hline Average ICU bed/ Hospital bed ratio, median [IQR] & $5.6[3.3,12.0]$ \\
\hline Average Occupancy Rate (\%), median [IQR] & $85[78,91]$ \\
\hline
\end{tabular}

${ }^{a}$ Average of SMR and SRU

b Includes surgical, neurological, and oncological critical care units

${ }^{\mathrm{C}}$ Average of day and night shifts in the unit

ICU: Intensive Care Unit

IQR: Interquartile Range (1st Quartile - 3rd Quartile)

Median and IQR are calculated from the distribution of values at unit level 
Table 5.3 -Confounder matrix

\begin{tabular}{|c|c|c|c|c|c|c|c|c|c|c|}
\hline & Organizational Characteristic & 1 & 2 & 3 & 4 & 5 & 6 & 7 & 8 & * \\
\hline 1 & Number of hospital beds & & & $\mathrm{x}$ & & $\mathrm{x}$ & & $x$ & & \\
\hline 2 & Ratio ICU beds per hospital bed & & & & & $\mathrm{X}$ & & & & $X$ \\
\hline 3 & Presence of medical residency in critical care & $X$ & & & $X$ & $x$ & $x$ & $x$ & $x$ & \\
\hline 4 & Specialized ICU b & $x$ & & $X$ & & & & & & \\
\hline 5 & Average Physicians per 10 beds $^{\text {a }}$ & $x$ & $X$ & & $X$ & & & $X$ & & \\
\hline 6 & $\begin{array}{l}\text { Presence of a full-time board-certified } \\
\text { intensivist }\end{array}$ & $X$ & & & $X$ & & & $x$ & & \\
\hline 7 & Average Nurses per 10 beds a & $x$ & $X$ & $X$ & $x$ & $X$ & $X$ & & & $X$ \\
\hline 8 & Admissions per bed & $\mathrm{x}$ & $\mathrm{x}$ & $x$ & $x$ & $\mathrm{x}$ & & $x$ & & \\
\hline
\end{tabular}

${ }^{a}$ Considering an average of day and night shifts in the unit

${ }^{\mathrm{b}}$ Includes surgical, neurological, and oncological critical care units

ICU: Intensive Care Unit

*: Characteristic is not considered as an exposure variable but only considered as potential confounder

" $\mathrm{X}$ ": Marks if a variable in the column is a potential confounder for the exposure variable in the rows

Regarding the multivariable analysis, we observed that both methods showed similar results (Table 5.4). The linear regression model with propensity score showed a statistically significant association between the average nurses per ten beds and ICU efficiency (ATE [95\% CI]: $-0.12[-0.20,-0.04]$ ). Similarly, the results from the Causal Random Forests showed a statistically significant association of the average nurses per ten beds with ICU efficiency (ATE [95\% CI]: -0.09 [-0.16, -0.02]). Besides, CRFs showed a significant association between the average number of physicians per ten beds and ICU efficiency (ATE [95\% CI]: $0.11[0.01,0.20])$.

Table 5.4 -Estimates of effect for each organizational factor to the efficiency in the linear model with propensity scores and the causal random forests

\begin{tabular}{|c|c|c|c|c|}
\hline & \multicolumn{2}{|c|}{ Main analysis (134 ICUs) } & \multicolumn{2}{|c|}{ Sensitivity analysis (131 ICUs) } \\
\hline & Regression model & $\begin{array}{c}\text { Causal Random } \\
\text { Forest }\end{array}$ & Regression model & $\begin{array}{c}\text { Causal Random } \\
\text { Forest }\end{array}$ \\
\hline Exposure & ATE $[95 \% \mathrm{Cl}]$ & ATE $[95 \% \mathrm{Cl}]$ & $\operatorname{ATE}[95 \% \mathrm{Cl}]$ & $\operatorname{ATE}[95 \% \mathrm{Cl}]$ \\
\hline $\begin{array}{l}\text { Number of hospital beds } \\
(x 100)\end{array}$ & $0.01[-0.02,0.04]$ & $-0.02[-0.07,0.03]$ & $0.003[-0.02,0.03]$ & $-0.04[-0.069,-0.001]$ \\
\hline $\begin{array}{l}\text { ICU beds per hospital beds } \\
\text { ratio }\end{array}$ & $0.002[-0.008,0.013]$ & $0.003[-0.007,0.013]$ & $0.006[-0.003,0.014]$ & $0.006[-0.002,0.015]$ \\
\hline $\begin{array}{l}\text { Presence of medical } \\
\text { residency in critical care }\end{array}$ & $0.06[-0.16,0.29]$ & $0.05[-0.09,0.20]$ & $0.10[-0.07,0.28]$ & $0.12[-0.02,0.26]$ \\
\hline Specialized ICU ${ }^{b}$ & $-0.10[-0.27,0.07]$ & $-0.04[-0.20,0.13]$ & $-0.06[-0.20,0.08]$ & $-0.08[-0.14,0.12]$ \\
\hline $\begin{array}{l}\text { Average Physicians per } 10 \\
\text { beds }^{\text {a }}\end{array}$ & $0.06[-0.07,0.19]$ & $0.11[0.01,0.22]$ & $0.02[-0.09,0.12]$ & $0.09[-0.01,0.19]$ \\
\hline Average Nurses per 10 beds $^{a}$ & $-0.12[-0.20,-0.04]$ & $-0.09[-0.16,-0.02]$ & $-0.08[-0.15,-0.01]$ & $-0.062[-0.121,-0.003]$ \\
\hline $\begin{array}{l}\text { Presence of a full-time board- } \\
\text { certified intensivist }\end{array}$ & $0.01[-0.13,0.15]$ & $-0.01[-0.14,0.11]$ & $0.02[-0.10,0.13]$ & $0.01[-0.10,0.13]$ \\
\hline $\begin{array}{l}{ }^{a} \text { Considering an average of d } \\
{ }^{b} \text { Includes surgical, neurologic } \\
\text { ATE: Average Treatment Effe } \\
\text { Cl: Confidence Interval } \\
\text { ICU: Intensive Care Unit } \\
\text { ASER: Average Standardized }\end{array}$ & $\begin{array}{l}\text { and night shifts in th } \\
\text {, and oncological criti } \\
\text { ffficiency Ratio }\end{array}$ & $\begin{array}{l}\text { unit } \\
\text { al care units }\end{array}$ & & \\
\hline
\end{tabular}


In the sensitivity analysis, we identified three outlying ICUs, which presented the highest ASER values (worst performing units). After removing those outliers, the new overall median SMR was 0.96 [IQR: 0.69, 1.24], median SRU was 1.03 [IQR: 0.82, 1.23], and median ASER was $0.99[0.82,1.25)$. We observed that both the linear regression model with propensity scores and the Causal Random Forest showed an association of the average nurses per 10 beds on the ICU efficiency (ATE [95\% CI]: -0.08 [-0.15, -0.01] and $-0.062[-0.121,-0.003]$, respectively) (Table 5.4). However, the CRFs did not a statistically significant association between the average number of physicians per ten beds and ICU efficiency.

\section{4 \\ Discussion}

This study examined the relationship between organizational factors and ICU efficiency in a large multicentre database of Brazilian ICUs. We found that the increase in average nurses per ten beds was associated with increased ICU efficiency in linear modelling and causal random forest after adjusting for other organizational variables. We also found that an increase in the average number of physicians per ten ICU beds was associated with decreased ICU efficiency.

This is not the first study that investigated the association between organizational factors and ICU efficiency in the Brazilian setting. At the patient level, Zampieri et al. identified that phenotypes of ICUs with high nursing autonomy, a full-time boardcertified intensivist, and a dedicated pharmacist might present better patient outcomes (ZAMPIERI et al., 2019). In Bastos et al., at the unit level, the ratio of nurses per bed per unit was not available, and staffing patterns were not significantly associated with efficiency in the final model (BASTOS et al., 2020b). Wortel et al. found that an increased number of intensivists per ICU bed was associated with increased ICU efficiency in Dutch ICUs (WORTEL et al., 2020). In their study, the number of nurses per patient was not associated with the efficiency outcome. This might be explained by the fact that the nurse to bed ratio was much higher in Dutch units (median 3.25 compared to 1.79 nurses per ten beds), and socioeconomic differences between the two countries may have a strong interplay with the organizational factors.

Our finding that ICU staffing is significantly associated with ICU efficiency is therefore not necessarily in contrast to earlier studies. Possibly our study setting showed 
enough heterogeneity among the ICUs to reveal the association. In particular, the presence of enough nurses in the ICU have led to better care of patients, with rapid response to adverse events and adherence to best practices (HIRZALLAH; ALKAISSI; DO CÉU BARBIERI-FIGUEIREDO, 2019; LARSON; MCKEEVER, 2018), especially when they are more autonomous in the ICU (ZAMPIERI et al., 2019).

In this study, we introduced the Causal Random Forests to estimate the average treatment effect of organizational factors on ICU efficiency. As far as we know, this is the first application of CRF to the analysis of ICU organizational data. This causal machine learning technique has received some attention recently as it provides confounder adjustment similar to stratification procedures, such as the k-nearest neighbours, and they are non-parametric (WAGER; ATHEY, 2018). The results from our sensitivity analysis showed that the regression model is more stable than the CRF. This can be explained by the fact that the organizational factor number of hospital beds and the number of physicians may have a nonlinear relationship, which became more apparent after removing the ICUs with extreme ASER values. However, this also indicates that the CRF is sensible to the data. Although this model does not have a minimum sample size, the tree-inducing procedure of CRF is largely impacted as the sample is split to induce trees and provide ATE estimates ('honesty splitting”) (ATHEY; TIBSHIRANI; WAGER, 2019; WAGER; ATHEY, 2018).

Our study has strengths and limitations. This study includes a large sample of Brazilian ICUs and patient admission data. Therefore, the results are a suitable representation of an average Brazilian ICU. Furthermore, we could confirm the results with an extra level of certainty with the application of multiple methods. As limitations, first, aside from bed occupancy rate, the organizational characteristics were not continuously collected during the study period, so changes over time were not considered. On the other hand, ICUs were asked to provide the organizational data that were most representative for the whole data collection period (2016-2018), so we believe that changes over time did not greatly affect the results. Second, we included a limited number of organizational characteristics in our analyses. These characteristics were mostly related to the ICU, but characteristics outside the ICU such as adherence to care protocols can also impact the efficiency in an ICU. Other ICU- and hospital-related factors were unfortunately not available in our study data. Third, by using the average SMR and SRU for measuring ICU efficiency, ICUs with a low SMR and high SRU (or vice versa) are considered equally efficient as ICUs with a low SMR and low SRU. We chose this method 
over the efficiency matrix described in previous literature (BASTOS et al., 2020b; RAPOPORT et al., 1994; ROTHEN et al., 2007; SOARES et al., 2015) to account for the loss of information. Fourth, directly comparing and measuring the linear modelling and causal random forest modelling performance was not possible because the ground truth is unknown. Therefore, the results of the models should not be interpreted as one approach is better than the other.

The results of our study could be useful for manager-clinicians, policy makers and health management in low and middle income countries as our results show that a higher number of nurses per ICU bed will pay off in terms of efficiency. Future studies may investigate whether there are potential ceiling effects to determine whether nurse and physician staffing ratios are efficient and whether results differ when benchmarking ICUs from countries with different economic or income status.

\section{5}

\section{Conclusion}

The regression modelling and the CRF method identified the number of nurses per bed as organisational factors associated with efficiency in a large sample of Brazilian ICUs. Our study showed that both methods are suitable to be applied to determine the effect of an organisational factor on ICU efficiency. However, it is also necessary to consider that observations with extreme values can impact the results, especially in the causal random forest. 


\title{
6 \\ Article 4 - Characterisation of the first 250000 hospital admissions for COVID-19 in Brazil: a retrospective analysis of nationwide data
}

This article was published at The Lancet Respiratory Medicine (2021)

\begin{abstract}
Background Most low-and-middle-income countries (LMICs) have little or no data integrated into a national surveillance system to identify characteristics or outcomes of COVID-19 hospitalisations and the impact of the epidemic on their national health systems. Our aim was to characterise the hospitalised COVID-19 adult patients in Brazil, together with the consequences of the burden of COVID-19 on the resources used and on in-hospital mortality.
\end{abstract}

Methods: We analysed hospitalised COVID-19 RT-qPCR-confirmed cases registered in the nationwide surveillance database (SIVEP-Gripe) in Brazil. We described the progression of the COVID-19 pandemic across three different periods, of four epidemiological weeks each (between 16/02/2020 and 15/08/2020). Our primary outcome was in-hospital mortality. We compared the regional burden of hospitalisations stratified by age, ICU admission and respiratory support. We analysed the whole country and its five geopolitical regions.

Findings: There were 254,288 RT-qPCR-confirmed COVID-19 hospitalisations in the SIVEP-Gripe (mean age $60 \pm 17$ years, $47 \%$ were under 60 years of age, $56 \%$ were male and $16 \%$ had no comorbidity). Among those with a defined hospital outcome (91\%, $232,036 / 254,288)$, the overall in-hospital mortality was $38 \%(87,515 / 232,036), 59 \%$ $(47,002 / 79,687)$ among those admitted in the ICU, and $80 \%(36,046 / 45,205)$ among those mechanically ventilated. The burden of hospitalisations in the North and Northeast was more pronounced than in the South and Southeast regions. In the Northeast, $15 \%$ $(1,545 / 9,960)$ of patients received invasive mechanical ventilation outside the ICU compared with $8 \%(431 / 5,338)$ in the South. In-hospital mortality among patients under 60 years old was $31 \%(4,204 / 13,468)$ in the Northeast compared with $15 \%(1,694 / 11,196)$ in the South. 
Interpretation: We observed a wide spread of the disease resulting in a high burden of COVID-19 in Brazil. In-hospital mortality was high, even in the young age groups, affecting mostly vulnerable populations and differently regional health systems. COVID19 pandemic highlights the challenge to secure access to optimized care for critically ill patients, particularly in low-and-middle income countries.

\section{1 Introduction}

Millions of COVID-19 cases have generated unprecedented stress on healthcare systems worldwide, including increased demand for hospitalisation, intensive care beds, advanced respiratory support, and trained healthcare professionals. The impact of the pandemic on each health system has been different, depending on the balance between supply and demand, which is associated with capacity to expand the health system and with pandemic preparedness.

Brazil is an upper-middle-income country with 210 million inhabitants in a large territorial area, in which there are significant regional differences. There is a remarkable heterogeneity between its five geopolitical regions (North, Northeast, Central-West, Southeast, and South), including cultural and socioeconomic aspects, reflected in health services, hospital beds, and healthcare worker availability. (AMARAL et al., 2017; MARINHO et al., 2018; SZWARCWALD et al., 2016) The pandemic, which was carried to Brazil by international flights, was initially concentrated in the large metropolitan areas, creating a spatial-temporal evolution from the capitals to the towns. (CANDIDO et al., 2020)

Recent economic and political crises have intensified structural problems in the Brazilian Universal Health System (SUS), including gaps in governance and organization, chronic underfunding, and low clinical effectiveness. (MARINHO et al., 2018; MASSUDA et al., 2018) The COVID-19 epidemic has challenged the Brazilian health system with more than 4.5 million cases and 140,000 deaths by the end of September 2020. (WORDOMETER, 2021) The existing regional disparities in access to health services and health outcomes were probably intensified by the pandemic, affecting the most vulnerable socioeconomic groups in the population.

Most low-and-middle-income countries (LMICs) have little or no data integrated into a national surveillance system to identify characteristics or outcomes of COVID-19 
hospitalisations and the impact of the epidemic on their national health systems. The Brazilian universal health system (SUS) and its informatics department (DATASUS) have a long tradition of acquiring and maintaining public records of health-related information for administrative and epidemiological purposes. (ALI et al., 2019) We aimed to describe the patient characteristics, intensive care use, and respiratory support of the first 250,000 hospitalised COVID-19 patients using a nationwide surveillance system in Brazil. Additionally, we tried to understand the consequences of the burden of COVID-19 for the resources used and the in-hospital mortality by analysing the five geopolitical regions in Brazil.

\section{2 \\ Methods}

\subsection{1}

\section{Study design and participants}

This study is a retrospective analysis of hospitalised adult patients with COVID-19 registered in the Influenza Epidemiological Surveillance Information System (Sistema de Informação de Vigilância Epidemiológica da Gripe, SIVEP-Gripe), a nationwide surveillance database used to monitor severe acute respiratory infections (SARI) in Brazil. (BASTOS et al., 2020a; DATASUS, 2020) Initially established in 2012, SIVEPGripe has been the primary source of information related to COVID-19 hospitalisations and deaths in the country. COVID-19 notification is compulsory in Brazil and SIVEPGripe receives notifications of COVID-19 hospitalised patients from both public and private sectors. In the period analysed in this study, COVID-19 hospitalized patients were from 4,407 Brazilian municipalities, totalizing $96 \%$ of population coverage (Appendix A6.1).

Each register includes individual information on patient demographics, selfreported symptoms and comorbidities, ICU admission and ventilatory support, as well as dates of symptoms onset, hospital admission, ICU admission, and in-hospital outcome (death or discharge). All data was publicly available after de-identifying patients and anonymising sensitive data (Appendix A4.1). Following ethically agreed principles on open data, this analysis does not require ethical approval in Brazil.

Our period of analysis was from epidemiological week 8 (starting 16/02/2020) until epidemiological week 33 (until 15/08/2020). We included all consecutive patients with a 
RT-qPCR SARS-CoV-2 positive result who had been hospitalised and were aged 20 years or older. SARS-CoV-2 diagnostic tests followed national and international standards and were conducted on certified laboratories. We excluded non-hospitalised patients, thus excluding patients who died outside the hospital. Information on data management is in the appendix (Appendix A4.1).

\section{2 .2}

\section{Other data sources}

We also show the total number (hospitalised and non-hospitalised) of confirmed SARS-CoV-2 cases at the municipal level reported by each state's Health Department, which is collected by the brasil.io consortium, a group of volunteers who compile daily epidemiological bulletins. (WORDOMETER, 2021) Brazilian population estimates for 2020 were retrieved from the Brazilian Institute of Geography and Statistics (IBGE), and numbers of active hospital and ICU beds from the National Registry of Health Establishments (CNES). (CNES - CADASTRO NACIONAL DE ESTABELECIMENTOS DE SAÚDE, 2020) A detailed description of the data sources is provided in the Appendix A4.1.

\subsection{3 \\ Outcomes}

Our primary outcome was in-hospital mortality. We also evaluated the use of resources (ICU admission and respiratory support, defined as none, non-invasive or invasive).

\subsection{4}

\section{Data analyses}

Our analysis was pre-specified and defined before any reading of the data. The sample size was pragmatic and defined by time: all adult hospitalised cases notified in the database between epidemiological week 8 and 33 .

We used the median and percentile $25-75$ or mean and standard deviation (SD) for continuous variables and calculated the frequency and proportions for categorical variables. We calculated age- and sex-adjusted rates for each regions by the direct method using the estimated Brazilian population for 2020 as reference.

We show the progression of the COVID-19 pandemic (total cases, hospitalisations and in-hospital deaths) throughout the country in three different periods, each one 
comprising four epidemiological weeks, to illustrate its spatial and temporal development: initial cases (weeks 8 to $12,16 / 02 / 2020$ to 21/03/2020), mid-term (weeks 19 to $22,03 / 05 / 2020$ to 30/05/2020), and the situation at the end of the analysed period (weeks 27 to 30,28/06/2020 to 25/07/2020). The first period comprised 4 weeks plus days of week 8 due to sparse data and the last period was censored until week 30 because of delayed entry of outcomes.

We evaluated in-hospital mortality and the use of resources in the health system for those patients who had already a hospital outcome. We compared the burden of the hospitalisations, the in-hospital mortality, and the proportion of resource use between regions. Burden was defined as the hospitalisation rate per 100,000 population. We calculated the in-hospital mortality by each region every four weeks period and estimated 95\% confidence intervals by the Agresti-Coull method. We also stratified the analysis by age, sex, number of comorbidities (comorbidities considered were cardiovascular, diabetes, renal, neurologic, hematologic, hepatic, chronic respiratory disorder, obesity, immunosuppression), level of education, self-reported race or skin colour (hereafter referred to as self-reported race), ICU admission and respiratory support. We conducted a sensitivity analysis by including also patients diagnosed by serological/antigen tests and clinical-epidemiological criteria, to account for potential selection bias towards severe cases because of the RT-qPCR tests prioritization.

Our main analysis was based on complete-case data, computing averages and proportions with the corresponding number of available data for each variable. However, the SIVEP-Gripe presents a considerable amount of missing information for some variables, such as reported symptoms and comorbidities. In a post-hoc analysis, we evaluated the missingness pattern and conducted a sensitivity analysis performing a multiple imputation by chained equations generating 30 imputed datasets. A description of the multiple imputation is shown in the Appendix A4.2.

Brazil is divided into five geopolitical regions: North, Northeast, Central-West, Southeast and South. These regions have historical differences in the capacity and coverage of the Brazilian health system. Thus, we performed analyses for the whole country and for each region. (XAVIER et al., 2019) All analyses were performed in R 4.0.2. Multiple imputation was performed in Stata 13.1. We followed STROBE guideline recommendations. 


\section{3}

\section{Results}

Between 16/02/2020 and 15/08/2020, there were 3,278,692 confirmed cases of COVID-19, spread over 5,506 (5,506/5,570, 99\%) municipalities in Brazil. During this period, 627,902 hospitalisations were reported in the SIVEP-Gripe (Figure 6.1). Of these hospitalisations, 254,288 adults tested positive for RT-qPCR for SARS-CoV-2.

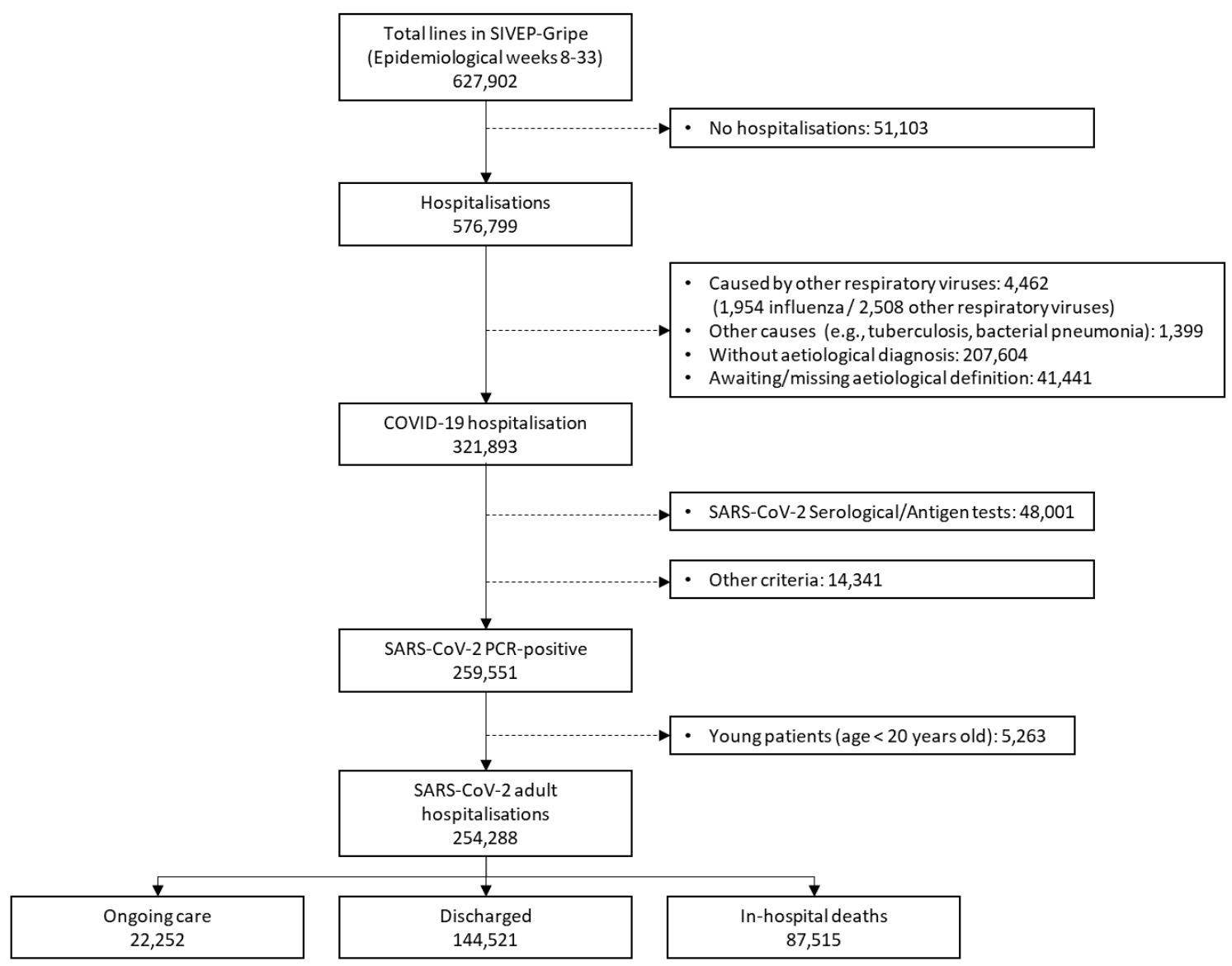

Figure 6.1 - Study flowchart

During epidemiological weeks 8-12, there were 1,092 confirmed cases and 773 hospitalisations in the 5 regions. This grew to 413,458 confirmed cases and 58,034 hospitalisations during the weeks 19-22, concentrated in the North, Northeast and Southeast. From week 27 to 30, there were 1,092,353 confirmed cases and 59,748 hospitalisations, concentrated in the Northeast and Southeast, but expanding to the Central-West and South regions (Figure 6.2). Crude and adjusted rates are in the Appendix A6.3. 

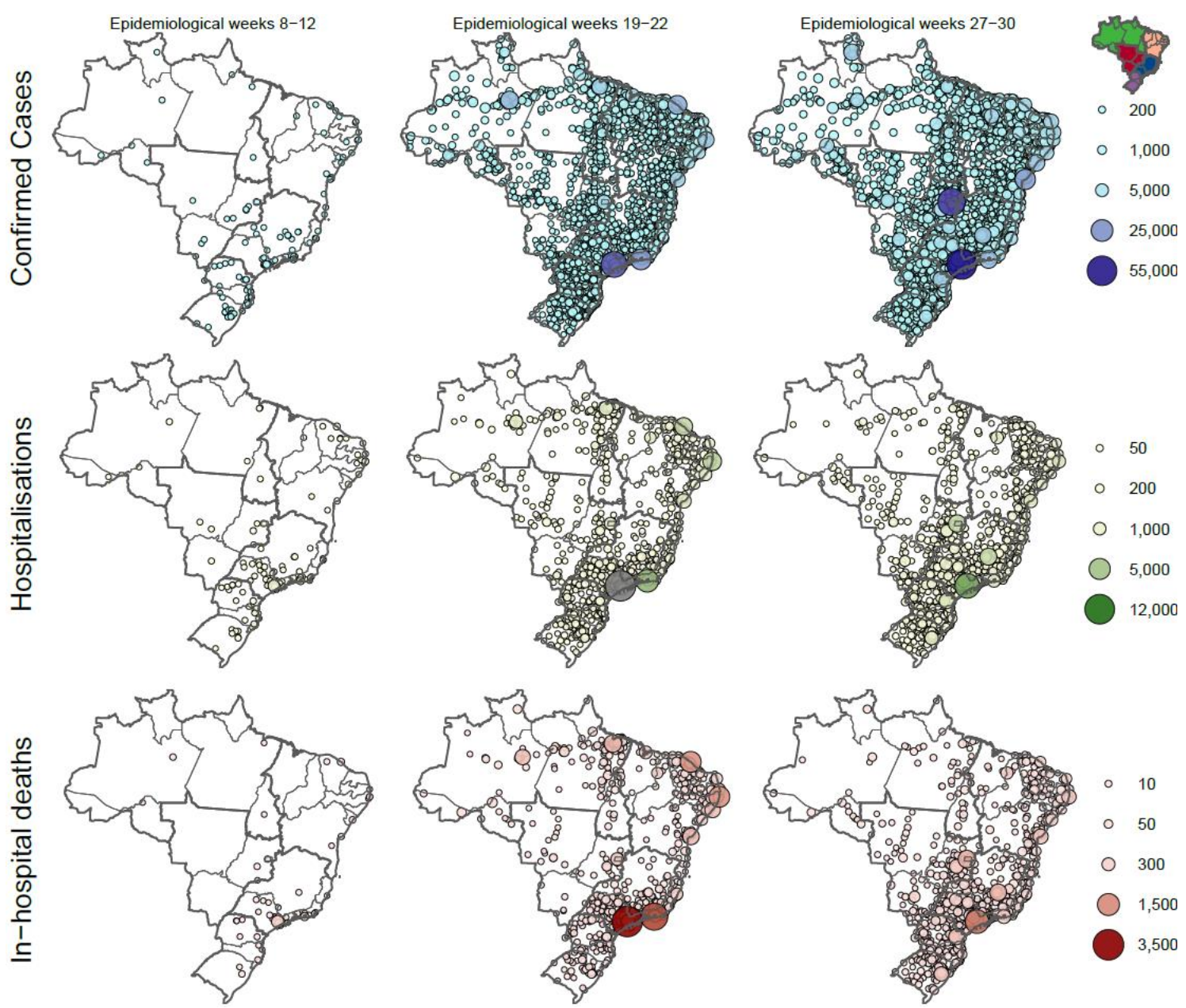

Figure 6.2 - Epidemic evolution shown in three-time frames in Brazil in terms of reported confirmed COVID-19 cases, hospitalisations and in-hospital deaths.

The maps display the municipalities in which cases, hospitalizations and deaths have been reported (points) and the volume (size). The numbers refer to what was observed within each time-frame.

The mean age was $60(\mathrm{SD}=17)$ years old, and there was a shift to older patients in Northeast region. Overall, White (49\%; 89,374/181,499) and Black/Brown (49\%; $88,773 / 181,499)$ patients were equally distributed, but Black/Brown accounted for more than two thirds of cases in the North, Northeast and Central-West regions. About one in five patients had no comorbidity and the median number of comorbidities was 1 [p25p75 1-2]. SARI was present in $61 \%(128,958 / 211,032)$ of patients and was more frequent in the North region (Table 6.1). Hypoxaemia (oxygen saturation <95\%) was present in $70 \%(147,596 / 212,016)$ of patients and was comparable between regions, while in the North and Northeast patients presented more frequently respiratory distress. Patient symptoms and comorbidities are described in the Appendix A4.3. 
Table 6.1 - Patient characteristics stratified by region

\begin{tabular}{|c|c|c|c|c|c|c|}
\hline Variables & $\begin{array}{c}\text { Brazil } \\
(n=254,288)\end{array}$ & $\begin{array}{c}\text { North } \\
(n=14,712)\end{array}$ & $\begin{array}{l}\text { Northeast } \\
(n=51,993)\end{array}$ & $\begin{array}{c}\text { Central- } \\
\text { West } \\
(n=18,701)\end{array}$ & $\begin{array}{l}\text { Southeast } \\
(n=142,963)\end{array}$ & $\begin{array}{c}\text { South } \\
(n=25,919)\end{array}$ \\
\hline $\begin{array}{l}\text { Age, mean (SD) }[\mathrm{n}= \\
254,288(100 \%)]\end{array}$ & $60(17)$ & $59(17)$ & $62(18)$ & $59(17)$ & $60(17)$ & $59(17)$ \\
\hline median (IQR) & $61(47,73)$ & $61(46,73)$ & $63(49,76)$ & $59(46,71)$ & $61(47,73)$ & $60(47,72)$ \\
\hline \multicolumn{7}{|l|}{ Age group, №. (\%) } \\
\hline $20-39$ & $34,170(13 \%)$ & 2,285 (15\%) & $6,672(13 \%)$ & $2,798(15 \%)$ & $\begin{array}{l}18,849 \\
(13 \%)\end{array}$ & $3,566(14 \%)$ \\
\hline $40-49$ & $37,618(15 \%)$ & 2,187 (15\%) & $6,566(13 \%)$ & $3,115(17 \%)$ & $\begin{array}{c}21,814 \\
(15 \%)\end{array}$ & $3,936(15 \%)$ \\
\hline $50-59$ & 47,869 (19\%) & $2,510(17 \%)$ & $8,742(17 \%)$ & $3,725(20 \%)$ & $\begin{array}{c}27,754 \\
(19 \%)\end{array}$ & $5,138(20 \%)$ \\
\hline $60-69$ & $52,800(21 \%)$ & $3,033(21 \%)$ & $\begin{array}{l}10,531 \\
(20 \%)\end{array}$ & $3,770(20 \%)$ & $\begin{array}{r}29,817 \\
(21 \%)\end{array}$ & $5,649(22 \%)$ \\
\hline $70-79$ & 44,968 (18\%) & 2,767 (19\%) & $\begin{array}{l}10,275 \\
(20 \%)\end{array}$ & 3,067 (16\%) & $\begin{array}{l}24,445 \\
(17 \%)\end{array}$ & $4,414(17 \%)$ \\
\hline $80+$ & $36,863(14 \%)$ & $1,930(13 \%)$ & $9,207(18 \%)$ & $2,226(12 \%)$ & $\begin{array}{c}20,284 \\
(14 \%)\end{array}$ & $3,216(12 \%)$ \\
\hline $\begin{array}{l}\text { Male sex, No. }(\%)[n= \\
254,243,99 \cdot 9 \%]\end{array}$ & $143,521(56 \%)$ & $8,816(60 \%)$ & $\begin{array}{c}28,983 \\
(56 \%)\end{array}$ & $\begin{array}{c}10,729 \\
(57 \%)\end{array}$ & $\begin{array}{l}80,340 \\
(56 \%)\end{array}$ & $\begin{array}{l}14,653 \\
(57 \%)\end{array}$ \\
\hline \multicolumn{7}{|c|}{ Self-reported race ${ }^{a}$, No. (\%) $[n=181,499(71 \%)]$} \\
\hline White & $89,374(49 \%)$ & $1,340(11 \%)$ & $5,515(17 \%)$ & $3,322(29 \%)$ & $\begin{array}{l}59,502 \\
(58 \%)\end{array}$ & $\begin{array}{l}19,695 \\
(88 \%)\end{array}$ \\
\hline Black/Brown & $88,773(49 \%)$ & $\begin{array}{c}10,039 \\
(86 \%)\end{array}$ & $\begin{array}{c}26,579 \\
(81 \%)\end{array}$ & $7,622(67 \%)$ & $\begin{array}{c}42,114 \\
(41 \%)\end{array}$ & $2,419(11 \%)$ \\
\hline Asian & $2,838(1 \cdot 6 \%)$ & $209(1 \cdot 8 \%)$ & $611(1.9 \%)$ & $265(2 \cdot 3 \%)$ & $\begin{array}{l}1,606 \\
(1.6 \%)\end{array}$ & $147(0 \cdot 7 \%)$ \\
\hline Indigenous & $514(0 \cdot 3 \%)$ & $121(1 \cdot 0 \%)$ & $95(0 \cdot 3 \%)$ & $164(1 \cdot 4 \%)$ & $87(<0 \cdot 1 \%)$ & $47(0 \cdot 2 \%)$ \\
\hline \multicolumn{7}{|c|}{ Level of education, No. (\%) $[n=86,204(34 \%)]$} \\
\hline Illiterate & $5,399(6 \cdot 3 \%)$ & $711(10 \%)$ & $1,682(14 \%)$ & $280(5 \cdot 4 \%)$ & $\begin{array}{l}2,250 \\
(4 \cdot 5 \%)\end{array}$ & $476(4 \cdot 1 \%)$ \\
\hline Up to high school & $38,417(45 \%)$ & $2,964(42 \%)$ & $5,203(42 \%)$ & $2,133(41 \%)$ & $\begin{array}{l}22,309 \\
(45 \%)\end{array}$ & $5,808(50 \%)$ \\
\hline High school & $28,365(33 \%)$ & $2,448(34 \%)$ & $3,629(29 \%)$ & $1,757(34 \%)$ & $\begin{array}{l}17,040 \\
(34 \%)\end{array}$ & $3,491(30 \%)$ \\
\hline College/University & $14,023(16 \%)$ & $981(14 \%)$ & $1,835(15 \%)$ & $1,006(19 \%)$ & $8,311(17 \%)$ & $1,890(16 \%)$ \\
\hline \multicolumn{7}{|c|}{ Number of comorbidities, No. $(\%)^{b}[n=90,829(36 \%)]$} \\
\hline 0 & $14979(16 \%)$ & $788(17 \%)$ & $2794(17 \%)$ & $1654(19 \%)$ & $7803(16 \%)$ & $1940(16 \%)$ \\
\hline $1-2$ & $67610(74 \%)$ & $3458(77 \%)$ & $\begin{array}{l}12088 \\
(75 \%)\end{array}$ & $6199(73 \%)$ & $\begin{array}{l}37051 \\
(75 \%)\end{array}$ & $8814(73 \%)$ \\
\hline$\geq 3$ & $8240(10 \%)$ & $271(6 \%)$ & $1221(8 \%)$ & $636(8 \%)$ & $4796(9 \%)$ & $1316(11 \%)$ \\
\hline $\begin{array}{l}\text { Oxigen saturation } \\
<95 \% \text {, No. (\%) }[\mathrm{n}= \\
212,016(83 \%)]\end{array}$ & $147,596(70 \%)$ & $7,955(67 \%)$ & $\begin{array}{l}27,410 \\
(69 \%)\end{array}$ & $\begin{array}{l}10,913 \\
(64 \%)\end{array}$ & $\begin{array}{l}85,739 \\
(71 \%)\end{array}$ & $\begin{array}{c}15,579 \\
(67 \%)\end{array}$ \\
\hline $\begin{array}{l}\text { Dyspnoea, No. (\%) }[\mathrm{n}= \\
226,724(89 \%)]\end{array}$ & $180,818(80 \%)$ & $\begin{array}{l}11,379 \\
(84 \%)\end{array}$ & $\begin{array}{c}36,883 \\
(83 \%)\end{array}$ & $\begin{array}{c}13,709 \\
(77 \%)\end{array}$ & $\begin{array}{l}99,548 \\
(79 \%)\end{array}$ & $\begin{array}{l}19,299 \\
(79 \%)\end{array}$ \\
\hline $\begin{array}{l}\text { Respiratory distress, } \\
\text { No. }(\%)[n=209,145 \\
(82 \%)]\end{array}$ & $143,977(69 \%)$ & $9,802(78 \%)$ & $\begin{array}{c}26,737 \\
(70 \%)\end{array}$ & $\begin{array}{c}11,286 \\
(66 \%)\end{array}$ & $\begin{array}{c}80,530 \\
(68 \%)\end{array}$ & $\begin{array}{l}15,622 \\
(67 \%)\end{array}$ \\
\hline $\begin{array}{l}\text { SARI criteria, No. }(\%) \\
{[n=211,032(83 \%)]}\end{array}$ & $128,958(61 \%)$ & $9,944(77 \%)$ & $\begin{array}{c}26,177 \\
(66 \%)\end{array}$ & $9,362(55 \%)$ & $\begin{array}{l}71,019 \\
(60 \%)\end{array}$ & $\begin{array}{c}12,456 \\
(54 \%)\end{array}$ \\
\hline $\begin{array}{l}\text { SARI without fever } \\
\text { criteria, No. (\%) [n = } \\
223,006(88 \%)]\end{array}$ & $171,574(77 \%)$ & $\begin{array}{l}11,274 \\
(85 \%)\end{array}$ & $\begin{array}{c}33,684 \\
(79 \%)\end{array}$ & $\begin{array}{l}12,520 \\
(72 \%)\end{array}$ & $\begin{array}{l}96,488 \\
(77 \%)\end{array}$ & $\begin{array}{c}17,608 \\
(73 \%)\end{array}$ \\
\hline $\begin{array}{l}\text { Hospitalization in state } \\
\text { capital, No (\%) }[\mathrm{n}= \\
254,288(100 \%)]\end{array}$ & $138,235(54 \%)$ & $9,018(61 \%)$ & $\begin{array}{c}36,339 \\
(70 \%)\end{array}$ & $\begin{array}{l}13,195 \\
(71 \%)\end{array}$ & $\begin{array}{l}71,411 \\
(50 \%)\end{array}$ & $8,272(32 \%)$ \\
\hline
\end{tabular}

The numbers and proportions within brackets refer to the available data for each variable.

SD - Standard deviation; SARI - Severe acute respiratory infection

a Race was collected as self-reported race or skin colour, originally classified as White (Branco), Black

(Preto), Brown (Pardo), Asian (Amarelo), and Indigenous (Indígena)

b Number of chronic comorbidities is the sum of the following comorbidites: cardiovascular, diabetes, renal, neurologic, hematologic, hepatic, chronic respiratory disorder, obesity, immunosuppression. 
A total of $232,036(91 \% ; 232,036 / 254,288)$ patients have had a hospital outcome when the data was exported, while 22,252 were still hospitalised.

The median time from onset of symptoms to hospitalisation was 6 [4-9] days in Brazil. There were 79,687 (39\%; 79,687/205,493) ICU admissions, with a median time from onset of symptoms to ICU admission of 7 [4-10] days (Table 6.2; Appendix A4.3). One in every four patients required invasive mechanical ventilation (23\%; $45,205 / 196,248)$, and 5,976 patients $(14 \% ; 5,976 / 44,055)$ received invasive mechanical ventilation outside the ICU (Table 6.2).

Table 6.2 - Intensive care admission, need of respiratory support, ICU and in-hospital mortality among patients with a defined hospital outcome $(n=232,036)$

\begin{tabular}{|c|c|c|c|c|c|c|}
\hline & $\begin{array}{c}\text { Brazil } \\
(n= \\
232,036)\end{array}$ & $\begin{array}{c}\text { North } \\
(n=13,496)\end{array}$ & $\begin{array}{l}\text { Northeast } \\
(n=45,238)\end{array}$ & $\begin{array}{c}\text { Central- } \\
\text { West } \\
(n=17,012)\end{array}$ & $\begin{array}{l}\text { Southeast } \\
(n=131,556)\end{array}$ & $\begin{array}{c}\text { South } \\
(n=24,734)\end{array}$ \\
\hline \multicolumn{7}{|c|}{ ICU, No. (\%) } \\
\hline $\begin{array}{c}\text { ICU } \\
\text { admission } \\
{[n=} \\
205,493 \\
(89 \%)]\end{array}$ & $\begin{array}{l}79,687 \\
(39 \%)\end{array}$ & 3,786 (32\%) & $14,867(43 \%)$ & $6,682(42 \%)$ & $45,224(38 \%)$ & $9,128(38 \%)$ \\
\hline $\begin{array}{l}\text { ICU } \\
\text { Mortality, } \\
\text { No. }(\%)^{\mathrm{a}}\end{array}$ & $\begin{array}{l}23,780 / 43 \\
582(55 \%)\end{array}$ & $\begin{array}{l}2,037 / 2,569 \\
(79 \%)\end{array}$ & $\begin{array}{l}4,834 / 7,357 \\
(66 \%)\end{array}$ & $\begin{array}{l}1,753 / 3,447 \\
(51 \%)\end{array}$ & $\begin{array}{c}11,058 / 22,472 \\
(49 \%)\end{array}$ & $\begin{array}{l}4,098 / 7,737 \\
(53 \%)\end{array}$ \\
\hline \multicolumn{7}{|c|}{$\begin{array}{l}\text { Respiratory support, No. (\%) }[\mathrm{n}=196,248 \\
(85 \%)]\end{array}$} \\
\hline None & $\begin{array}{c}54,314 \\
(28 \%)\end{array}$ & 3,047 (28\%) & $8,177(25 \%)$ & $4,076(27 \%)$ & $32,756(29 \%)$ & $6,258(27 \%)$ \\
\hline $\begin{array}{l}\text { Yes, non- } \\
\text { invasive }\end{array}$ & $\begin{array}{c}96,729 \\
(49 \%)\end{array}$ & $4,743(43 \%)$ & $14,485(44 \%)$ & $7,561(49 \%)$ & $58,444(51 \%)$ & $11,496(50 \%)$ \\
\hline \multicolumn{7}{|c|}{$\begin{array}{l}\text { Place of non-invasive respiratory support, No. }(\%)^{\mathrm{b}} \quad[n=91,816 \\
(95 \%)]\end{array}$} \\
\hline In ICU & $\begin{array}{c}27,236 \\
(30 \%)\end{array}$ & $695(15 \%)$ & $3,899(29 \%)$ & $2,359(32 \%)$ & $16,930(31 \%)$ & $3,353(30 \%)$ \\
\hline Outside & $\begin{array}{l}64,580 \\
(70 \%)\end{array}$ & 3,889 (85\%) & $9,675(71 \%)$ & $4,904(68 \%)$ & $38,138(69 \%)$ & $7,974(70 \%)$ \\
\hline $\begin{array}{l}\text { Yes, } \\
\text { invasive }\end{array}$ & $\begin{array}{c}45,205 \\
(23 \%)\end{array}$ & $3,155(29 \%)$ & $10,322(31 \%)$ & 3,667 (24\%) & $22,648(20 \%)$ & $5,413(23 \%)$ \\
\hline \multicolumn{7}{|c|}{ 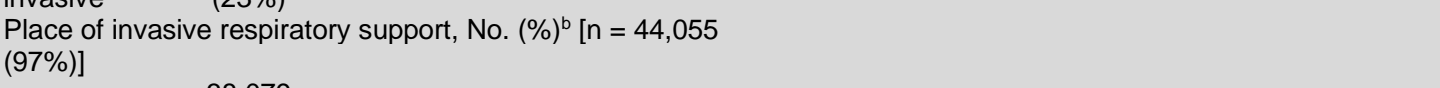 } \\
\hline In ICU & $\begin{array}{l}38,079 \\
(86 \%)\end{array}$ & 2,577 (83\%) & 8,415 (84\%) & 2,970 (83\%) & $19,160(87 \%)$ & 4,957 (92\%) \\
\hline $\begin{array}{l}\text { Outside } \\
\text { ICU }\end{array}$ & $\begin{array}{l}5,976 \\
(14 \%)\end{array}$ & $516(17 \%)$ & $1,545(16 \%)$ & $629(17 \%)$ & 2,855 (13\%) & $431(8 \%)$ \\
\hline \multicolumn{7}{|c|}{ Hospitalisation } \\
\hline $\begin{array}{l}\text { Hospital } \\
\text { mortality, } \\
\text { No. (\%) } \\
{[n=232,036} \\
(100 \%)]\end{array}$ & $\begin{array}{l}87,515 \\
(38 \%)\end{array}$ & $6,727(50 \%)$ & $21,858(48 \%)$ & $5,964(35 \%)$ & 45,269 (34\%) & $7,697(31 \%)$ \\
\hline \multicolumn{7}{|l|}{$\begin{array}{l}\text { Length-of- } \\
\text { stay, } \\
\text { median } \\
\text { (IQR) } \\
\text { ICU }\end{array}$} \\
\hline $\begin{array}{l}\text { Hospital } \\
{[n=218,281} \\
(94 \%)]\end{array}$ & $8(4,14)$ & $7(4,14)$ & $8(4,16)$ & $8(4,14)$ & $8(4,14)$ & $8(4,15)$ \\
\hline
\end{tabular}

The numbers and proportions in brackets refer to the available data for each variable.

ICU - intensive care unit 
a ICU mortality was derived for patients with date of ICU discharge equals to the date of the hospital death, so it was available for patients without missing values on both dates $(n=43,582)$

b The sum of non-invasive and invasive respiratory support when stratified by place - in ICU and outside ICU - does not match the total respiratory support type because of missing values on the variable ICU admission

The overall in-hospital mortality was $38 \%(87,515 / 232,036)$, with a steep increase with age $(12 \%, 3,780 / 30,603$ for the group 20-39 years; $27 \%, 11,818 / 43,376$ for those $50-59$; and $66 \%, 22,787 / 34,385$ for those above 80 years); it was slightly higher for males than females (Figure 6.3; Appendix A4.3). The in-hospital mortality of those without comorbidities was 32\% (4,494/13,836) (Figure 6.3; Appendix A4.3). There was an increase in the proportion of in-hospital deaths among those illiterate $(63 \%, 3,146 / 4,993)$, Black/Brown (43\%, 34,345/80,392) and Indigenous (42\%, 202/477) (Figure 6.3; Appendix A4.3). In-hospital mortality was higher for those admitted to the ICU (59\%, $47,002 / 79,687)$ than those admitted to the ward $(23 \%, 29,361 / 125,806)$. It was also higher for those invasively mechanically ventilated $(80 \%, 36,046 / 45,205)$ than those not invasively mechanically ventilated $(24 \%, 36,942 / 151,043)$. In-hospital mortality for patients aged 20-39 years who required mechanical ventilation was 57\% $(1,858 / 3,278)$ and for those above 60 years was $87 \%(25,879 / 29,853)$ (Figure 6.5; Appendix A4.3). Inhospital mortality was higher for patients who presented with hypoxaemia (45\%, $60,583 / 135,620)$, respiratory distress $(43 \%, 56,730 / 132,188)$ and dyspnoea $(41 \%$, 68,083/165,977) (Appendix A4.3). ICU mortality $(55 \%, 23,780 / 43,582)$ followed the same pattern as in-hospital mortality among regions (Table 6.2). 

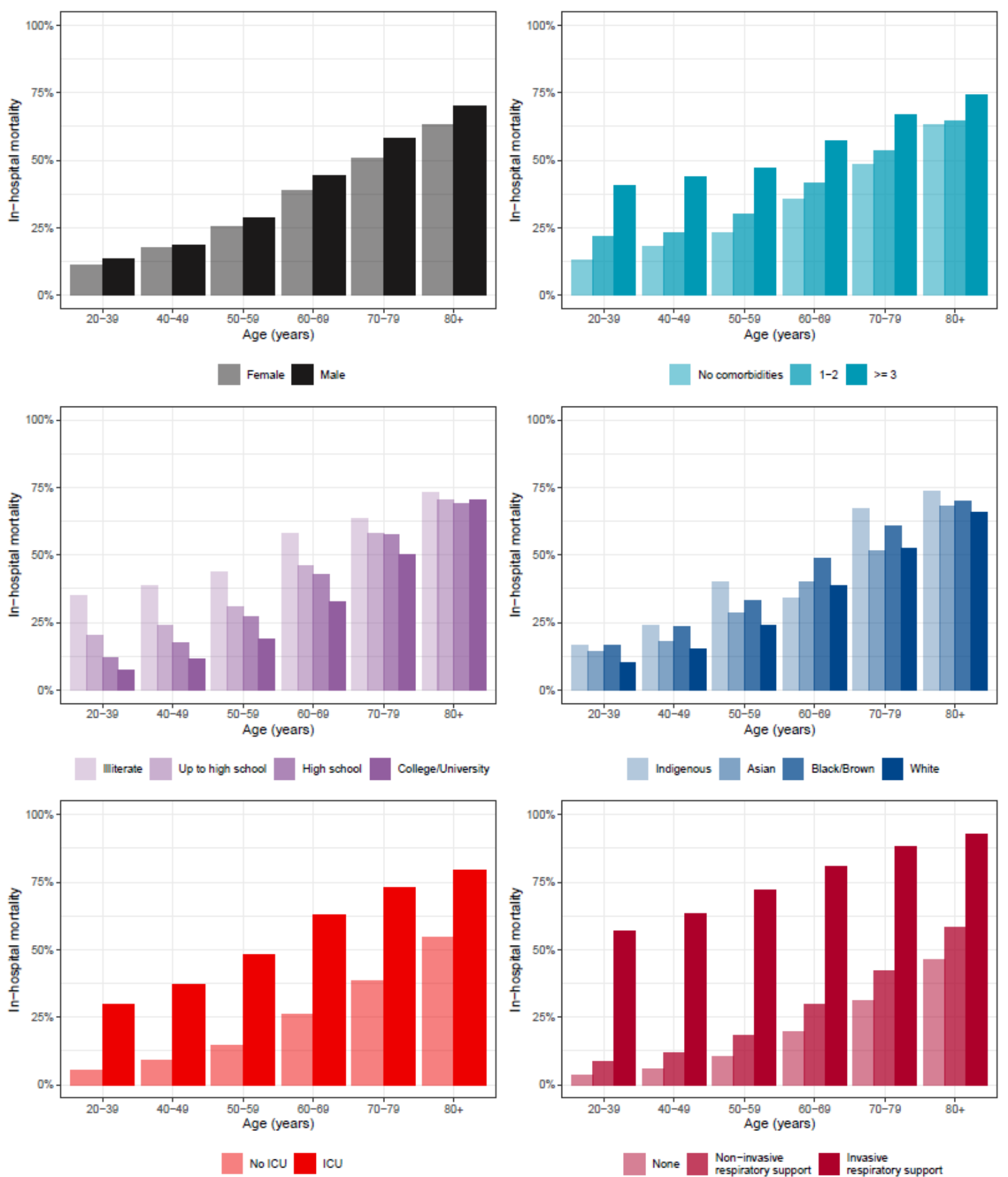

Figure 6.3 -In-hospital mortality stratified by age, sex, comorbidities, level of education, selfreported race ${ }^{*}$, intensive care admission and invasive mechanical ventilation for hospitalised COVID-19 patients in Brazil.

Data refers to patients with a defined hospital outcome and proportions were calculated based on complete-case data for sex, comorbidities, level of education, self-reported race, ICU and invasive ventilation variables. ${ }^{*}$ Race was collected as self-reported race or skin colour, originally classified as White (Branco), Black (Preto), Brown (Pardo), Asian (Amarelo), and Indigenous (Indígena)

The general characteristic of each region is shown on Table 6.3. There was a great difference in number of hospital and ICU beds between regions and between capitals and towns. The rate of hospitalisations for COVID-19 was 153 per 100,000 inhabitants in Brazil, considering patients with a defined hospital outcome (Appendix A4.3). When 
analysing over time, there was a different pattern of the hospitalization rates between regions. The crude in-hospital mortality was higher during weeks with high hospitalization rate, particularly for the North, Central-West and South region (Figure 6.4).

Table 6.3 - Demographic, administrative and health system regional characteristics

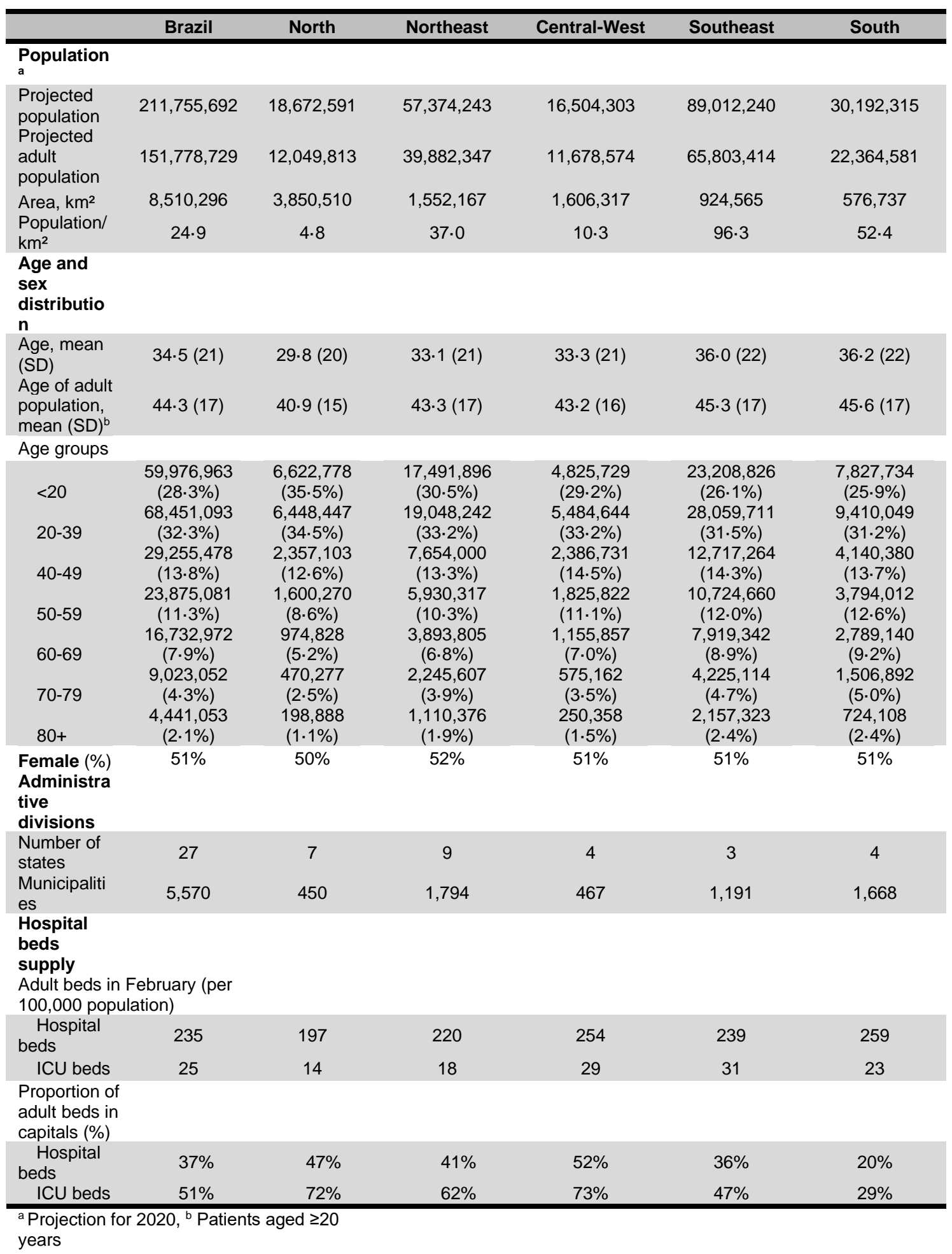



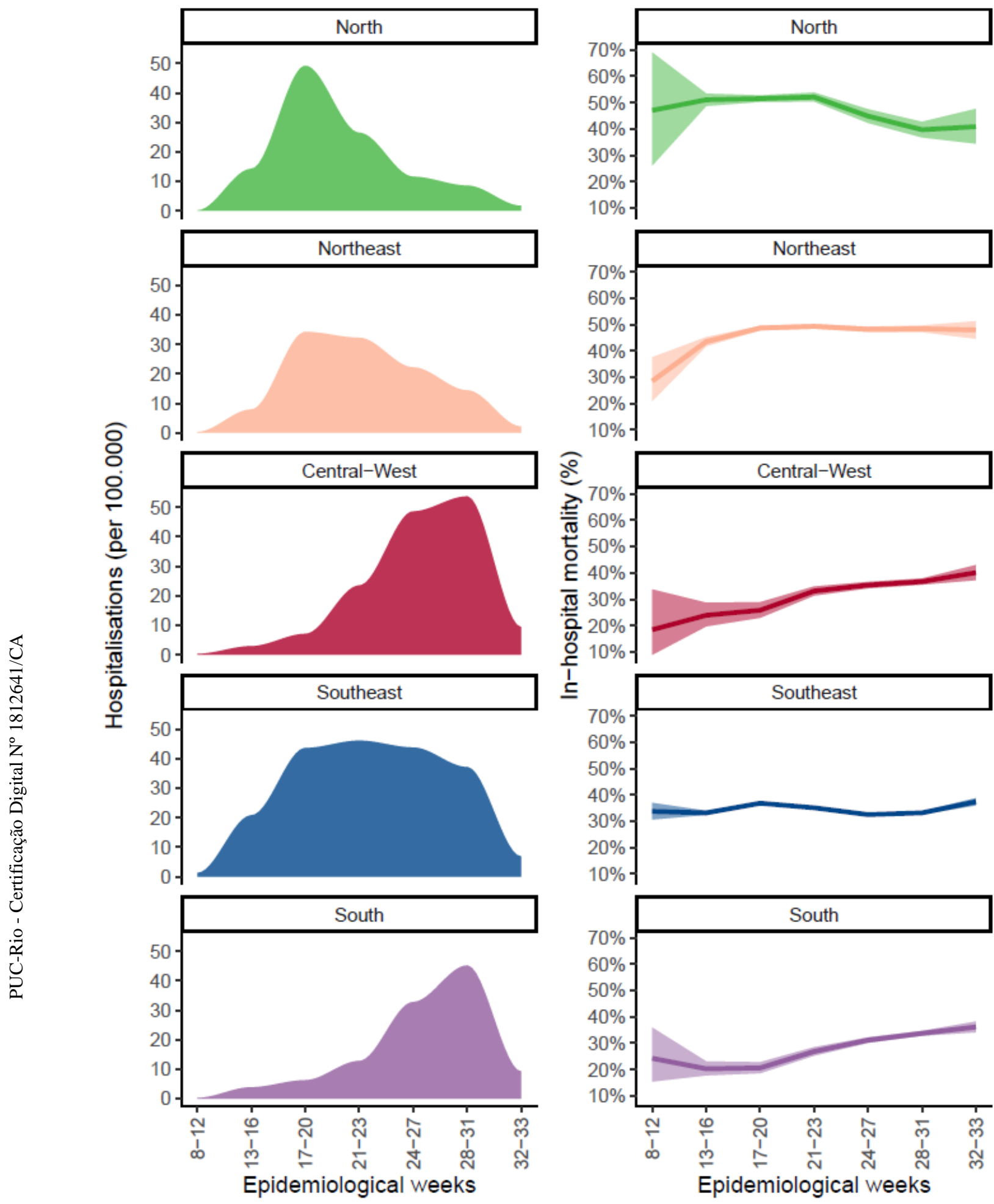

Figure 6.4 -Temporal evolution of COVID-19 hospitalisation rates per 100,000 adult population and crude in-hospital mortality in the five geopolitical regions of Brazil.

Epidemiological weeks on $\mathrm{x}$-axis refer to the onset of symptons. Shaded areas correspond to the upper and lower $95 \%$ confidence intervals estimated by the Agresti-Coull method.

When analysing the entire period, there were noticeable regional differences in hospitalisation rates, particularly when stratified by age (Figure 6.5). The North region had the highest COVID-19 hospitalisation incidence among patients over 70 years of age, 
followed by the Southeast, Central-West and Northeast. A similar pattern was observed for invasive mechanical ventilation (Figure 6.5; Appendix A4.3). When considering ICU admissions per ICU bed, the North region had the highest rate (2,246/1,000 ICU beds; Appendix A4.3). Most patients were hospitalised in the capital cities (54\%, $138,235 / 254,288)$, but this proportion was lower for the South $(32 \%, 8,272 / 25,919)$ and higher for the North, Northeast and Central-West regions (Table 6.1).

In-hospital mortality was higher for the North and Northeast regions in general (Table 6.2) and stratified by age (Figure 6.5; Appendix A4.3). Among those aged 20-39, the in-hospital mortality for the North was $20 \%(393 / 1,976)$ and $19 \%(1,083 / 5,587)$ for the Northeast. For the same age category, it was $10 \%(1,736 / 17,170)$ in the Southeast and $8 \%(284 / 3,372)$ in the South. The difference across regions was greater for patients under 50 years of age that were admitted to the ICU or mechanically ventilated (Figure 6.5; Appendix A4.3).
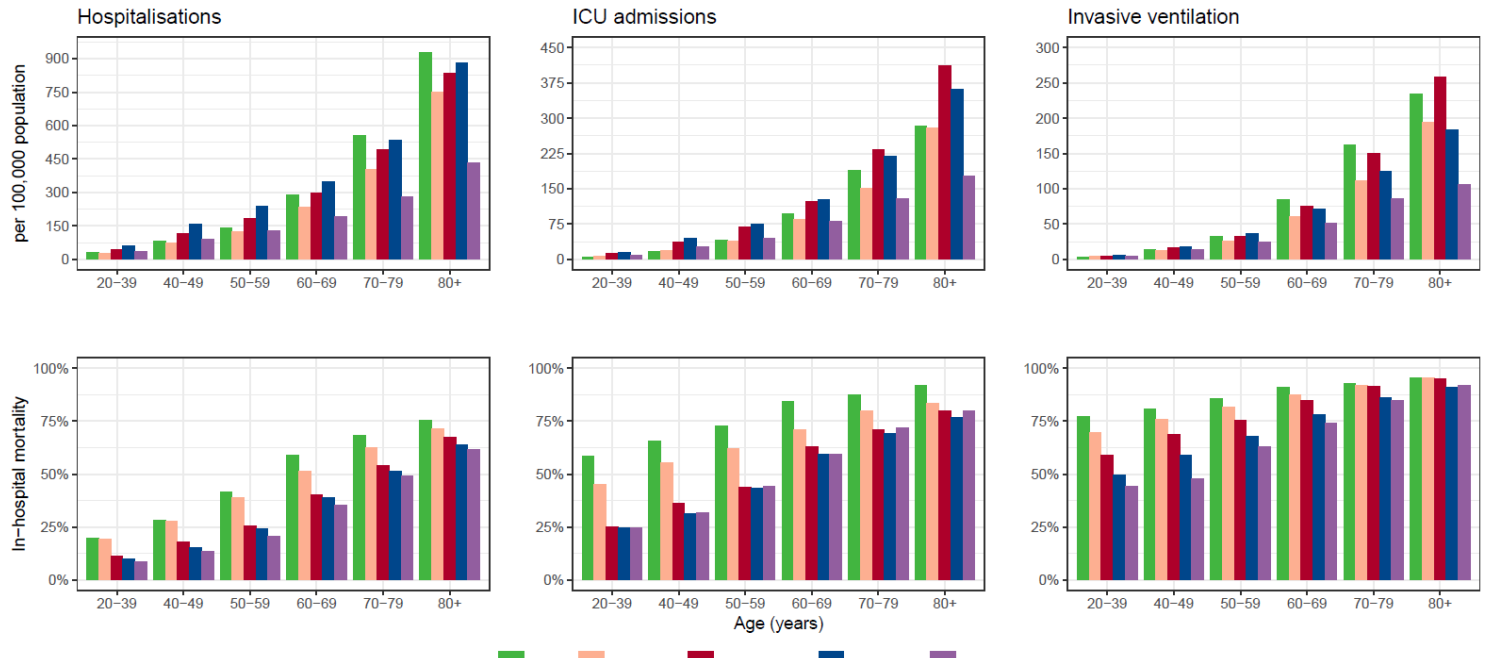

Figure 6.5 -Health system burden and in-hospital mortality stratified by age in hospitalised COVID-19 patients in the five regions of Brazil.

Burdens is defined as the hospitalisation rate per 100,000 population of each region (first row), and in-hospital mortality is the proportion of in-hospital deaths (second row). Data refers to patients with a defined hospital outcome and proportions were calculated based on completecase data for ICU and invasive mechanical ventilation variables.

The pattern of hospital resources was different between regions (Appendix A4.3). Overall, there was an increase in the proportion of ICU admission and of invasive mechanical ventilation with age. However, there was a plateau in the proportion of patients admitted to the ICU and in invasive mechanical ventilation in those over 60 years of age in the North region. Additionally, the proportion of patients who were admitted to 
the ICU and mechanically ventilated was comparable across ages in the North region; however, the proportion of patients admitted to the ICU was greater than those receiving invasive mechanical ventilation in the other regions (Appendix A4.3).

When considering COVID-19 hospitalised patients defined by clinical and laboratorial diagnosis, there were 314,615 patients and the majority of added patients $(\mathrm{n}=60,327)$ were from the North $(27,502 / 14,712$, a relative increase of $87 \%)$ and Northeast $(71,442 / 51,993$, relative increase of $37 \%)$. Overall, the characteristics of the patients were similar to those patients confirmed by RT-qPCR (Appendix 6.4). When analysing those with a defined hospital outcome $(n=284,747)$, the in-hospital mortality was the same at 38\% (108,566/284,747) (Appendix 6.4), although it was slightly lower for the North region $(44 \%$; 11,099/25,061 versus 50\%; 6,727/13,496). Overall, the same pattern of in-hospital mortality by age, number of comorbidities, level of education, selfreported race, ICU admission and invasive mechanical ventilation was observed, as well as when stratified by region (Appendix A4.4). The burden in the health system of the North region compared with other regions were more pronounced than in the main analysis (Appendix A4.4).

Overall, the analysis on multiple imputed data showed comparable results and we observed only two differences. The proportion of patients with $\geq 3$ comorbidities increased from $9 \%$ in complete case to $26 \%$ in multiple imputed data (Appendix A4.4). This change reflected in the observed in-hospital mortality when stratified by number of comorbidities, particularly for young patients (e.g., $40 \%$ in complete case to $19 \%$ in multiple imputed data for patients with $\geq 3$ comorbidities; Appendix A4.4). Other difference was for mechanically ventilated patients in the 20-39 age category in the Northeast region, with a decrease from $70 \%$ in-hospital mortality in complete case to $65 \%$ in the imputed analysis (Appendix A4.4).

\section{4 \\ Discussion}

We described the surge of hospitalised COVID-19 adult cases during the first five months of the pandemic in Brazil, using a nationwide database covering each geopolitical region. We analysed more than 250,000 cases with a mean age of 60 years. Of these, $16 \%$ had no comorbidity, and $72 \%$ received some respiratory support. We observed high in- 
hospital mortality, even among young patients, and substantial regional differences in terms of resources available and observed outcomes.

The overall in-hospital mortality was $38 \%$, which is comparable to other national cohorts (Appendix A4.5). However, if we consider that the analysed population is, on average 10 years younger (47\% aged $<60$ years) than that analysed in large European series, (DOCHERTY et al., 2020; GRASSELLI et al., 2020; KARAGIANNIDIS et al., 2020) the in Brazil mortality is noticeably higher. When the pandemic started, the first impression was that LMICs might be less affected as they have a younger populations than high-income countries. (CENTRE FOR GLOBAL INFECTIOUS DISEASE ANALYSIS, IMPERIAL COLLEGE LONDON, 2020) However, we observed high mortality even in young patients ( $<60$ years, 20\%; Appendix A4.3). In a nationwide study of 23,367 hospitalised patients with a defined hospital outcome in Iran, the cumulative risk of death in 30 days was $24 \%$ overall and $42 \%$ for those $\geq 65$ years. (JALILI et al., 2020) In a nationwide study in Germany, (KARAGIANNIDIS et al., 2020) 17\% $(1,727 / 10,021)$ of patients received mechanical ventilation (invasive or non-invasive), the in-hospital mortality was $22 \%(2,229 / 10,021)$, and 5\% $(135 / 2,896)$ for those under 60 years of age. In Mexico, the in-hospital mortality was $74 \%(8,861 / 12,018)$ among mechanically ventilated patients. (NAMENDYS-SILVA; GUTIÉRREZ-VILLASEÑOR; ROMERO-GONZÁLEZ, 2020) Comparisons with other cohorts are challenging because of the lack of nationwide data and of international standard criteria for severity, need for hospitalisation, and case definition. Although different criteria for hospital admission and other patient characteristics (e.g., comorbidities) could explain some of these differences between countries, the mismatch between demand and supply leading to a collapsed system could in part explain the increased in-hospital mortality in Brazil. (FREITAS et al., 2020; LEMOS et al., 2020; ORELLANA et al., 2020)

Several factors relate to differences observed in mortality and resource use among the Brazilian regions during the pandemic. These include the existing regional heterogeneity of the health system, followed by the temporal distribution of cases, and the adhesion to best practices of clinical management of severe patients. Despite the high absolute number of hospitals and ICU beds in the country compared with western European countries, (AUSTIN et al., 2014; SALLUH; LISBOA, 2016) the heterogeneous regional distribution is a significant barrier to more equitable access to these resources. The North and Northeast regions have the lowest hospital and ICU beds per capita in Brazil. This difference is even more pronounced when analysing ICU beds: the Southeast 
had two times more ICU beds per capita than the North region at the beginning of the pandemic in Brazil (Table 6.3). Additionally, ICU beds are concentrated in state capitals and the coastal regions (Table 6.3), (AZEVEDO et al., 2013; MACHADO et al., 2017) generating an additional barrier to access to the health system, especially after the pandemic evolved inland. The regional differences were also reflected in the proportion of hospitalised patients in state capitals, which was noticeably lower in the South and Southeast regions (Table 6.1), likely reflecting a better distribution of health services across these regions.

The surge affected the Southeast, North and Northeast early (Figure 6.2, Figure 6.4; Appendix A4.3), and these two last regions have more fragile medical systems. A national study on the prevalence of COVID-19 antibodies (HALLAL et al., 2020) identified a rapid initial escalation in Brazil's North and Northeast regions, strongly associated with Indigenous ancestry and low socioeconomic position. Although they have youthful populations, the in-hospital mortality was even higher in the North and Northeast regions than in other areas, with an increasing number of patients who required ICU admission and invasive ventilation. For mechanically ventilated patient under 60 years old, the mortality was $77 \%(2,559 / 3,317)$ in the Northeast compared with $55 \%$ $(1,054 / 1,929)$ in the South. The high proportion of mechanically ventilated patients in the ICU, the number of patients ventilated outside the ICU and the potential limitation of advanced respiratory support and ICU admission for those above 60 years of age reflect the stress and strain observed in these regions.

Other studies that evaluated severe patients admitted to ICUs in Brazil prior to the pandemic have shown high in-hospital mortality. In a large national survey which analysed patients who received invasive or non-invasive mechanical ventilation for at least 24 hours, the in-hospital mortality was $42 \%$. It was $52 \%$ for those with acute respiratory distress syndrome (ARDS), (AZEVEDO et al., 2013) which is present in a considerable proportion of hospitalised COVID-19 patients. (TZOTZOS et al., 2020) A nationwide study evaluating patients admitted to Brazilian ICUs with sepsis (61\% having the lung as the main source of infection) found the in-hospital mortality was $56 \%$ and there was an association between hospital resources and in-hospital mortality. (MACHADO et al., 2017) In a recent study of severe community-acquired pneumonia patients hospitalised in ICUs of public hospitals in Brazil, the in-hospital mortality was 66.7\% . (ESPINOZA et al., 2019) These data indicate a high mortality rate in critically ill patients in Brazil prior to the COVID-19 surge, especially among those who were 
ventilated. The stress on the system in the low-resource regions during the pandemic has likely accentuated this scenario.

Outcomes of critically-ill patients - such as hospitalised COVID-19 patients - are determined not only by resources and devices. Organisational factors and implementation of the best practice available result not only in better outcomes, such as mortality, but better ICU efficiency. Previous analyses of Brazilian ICUs have shown that there is room for improvement in adherence to best practices such as target sedation levels, low tidal volume ventilation,(MIDEGA et al., 2020; NASSAR et al., 2019) and active surveillance of nosocomial infections. (BASTOS et al., 2020b; SOARES et al., 2015) These practices are all associated with better outcomes. The data presented here reinforce the picture of the heterogeneity of care delivered to severe patients in LMICs. The good care provided in some high-end hospitals contrasts sharply with most facilities, which frequently provide lower quality of care.

In LMICs, health systems are commonly stretched in terms of resources and staff, and the early containment of a pandemic has tremendous advantages, leading to lower numbers of cases and hospitalisations which, in turn, allows time for expansion of bed numbers, staff training, and resources. (SONENTHAL et al., 2020) However, during the current pandemic response, much attention was dedicated to available resources such as ICU beds and ventilators, but little to training health professionals in the best evidence or the early identification of severe cases or clinical management of ventilated patients. Additionally, the presence of universal health coverage (UHC) is a fundamental strategy to make sure that everyone has access to testing or treatment without financial hardship. However, a coordinated national response, increasing the system's resilience to prevent its collapse, and clear communication of best practices are essential to reduce preventable deaths, especially in the young population in LMICs.

This study has limitations. First, the notification of COVID-19 hospitalisations is compulsory, but we cannot guarantee $100 \%$ coverage of all hospitalised cases in Brazil. However, the total population of the municipalities with at least one hospitalised patient included in this analysis comprises more than $96 \%$ of the Brazilian population. We would expect that during the initial phase of the pandemic more severe cases were notified, overestimating the in-hospital mortality. Nonetheless, SIVEP-Gripe is the official national database and is used to count hospitalised cases and all deaths related to COVID19; therefore we did not expect any important reporting bias. Second, there are regional differences in access to resources such as RT-qPCR tests. Particularly in the North and 
Northeast, a greater number of COVID-19 patients were diagnosed by serological/antigen tests and clinical-epidemiological criteria. In our sensitivity analysis, we observed a relatively greater increase in the number of patients in these regions and a slight decrease in the in-hospital mortality (Appendix A4.4). Third, changes and improvements in clinical practices probably occurred in relation to COVID-19 over time, but the assessment of temporal changes affecting in-hospital mortality is beyond the scope of this study. Fourth, this study is a descriptive analysis stratified by age and region and does not aim to answer causal questions considering several potential confounding factors and the dynamics of the pandemic. Therefore, we did not adjust for some patient characteristics (e.g., malnutrition), treatment (e.g., antivirals), health system (e.g., public vs. private sectors, ICU beds expansion), and regional characteristics (e.g., inequity and economic development). Finally, $9 \%$ of patients were still hospitalised at the time the database was exported, and the database could be updated at a later stage, but we have no indication that the outcome of these patients would change the main message of the current analysis.

In the analyses of this large nationwide database of confirmed COVID-19 cases, we demonstrated the dynamics of the surge of COVID-19 cases in Brazil, their clinical and demographic characteristics, how regional inequities impact the outcomes, and the collapse of the more fragile regional health systems during the pandemic. In-hospital mortality was high, even in the young age groups, particularly among those who were mechanically ventilated. 


\section{Article 5 - Severity, resource use and outcomes of COVID-19 hospital admissions in Brazil: comparison between the first and second wave}

\section{1 \\ Correspondence}

Brazil is one of the countries most affected by the COVID-19 pandemic, with more than 16 million confirmed cases and 454,429 confirmed deaths by May 26, 2021 (DONG; DU; GARDNER, 2020). Recently, Brazil is facing a synchronized epidemic growth, contrasting with the first surge (RANZANI et al., 2021). The beginning of 2021 is thus marked by simultaneous explosive waves of COVID-19 cases across different country regions, pressuring the health system overload by one year of pandemic. This surge is contemporary with the discovery, expansion, and dominance of variants of interest (VoI) and concern (VoC) in Brazil (FARIA et al., 2021).

We previously characterised the first 250,000 hospitalisations by COVID-19 in Brazil and its impact on resource use and in-hospital mortality.(RANZANI et al., 2021) We now compared the burden, severity (number of hypoxaemia patients), resource use (intensive care unit [ICU] admissions and respiratory support), and mortality of COVID19 hospitalised patients between the first and second wave. We extracted from the nationwide surveillance database (SIVEP-Gripe, https://opendatasus.saude.gov.br, 1,217,332 COVID-19 hospital admissions from 16/02/2020 to 24/05/2021 (epidemiological weeks $8 / 2020$ to $21 / 2021$ ). For the quantitative comparison between waves, we censored the last 4 weeks to account for a potential delay in notifications, resulting in 1,187,840 hospital admissions.

The hospital admissions dynamic showed a second surge after week 43/2020, defined by the lowest value per week of hospitalized cases (Figure 7.1). Comparing the second to the first wave (weeks 8/2020 to 21/2021), average admissions per week increased 59\% (14,220 vs 22,703, Table 7.1), with 72\% more patients with hypoxaemia $(8,606 \mathrm{vs} 14,845$ per week). The demand for non-invasive ventilation (NIV) increased $74 \%$ (6,746 vs 11,773 admissions per week) and $53 \%$ for invasive mechanical ventilation 
(IMV) per week (2,452 vs 3,747). Remarkably, the maximum number of admissions per week requiring advanced respiratory support (NIV+IMV) was 13,985 (week 28/2020) in the first wave, which turned to be 40,797 (week 10/2021) in the second wave (relative increase of 192\%). Although more patients with hypoxaemia underwent respiratory support, there was no increase in the proportion of patients admitted to the ICU $(37 \cdot 6 \%$ vs. $37.5 \%$ ), which suggests a potential limitation in the access to critical care (Table 7.1). Interestingly, in the second wave there were less admissions from State capitals than in the first $(48 \cdot 2 \%$ vs $37 \cdot 5 \%)$.

Within the second wave, on week 53, the E484K mutation among SARS-CoV-2 variants in Brazil was present in more than $50 \%$ of the viral genomes (S:E484K Mutation Report, outbreak.info, accessed May 26, 2021) (WISE, 2021) motivating specific comparisons of admissions before and after dominance of $\mathrm{VoI} / \mathrm{VoC}$. The median age of patients decreased (63 vs. 59 years-old), with a relative increase of $18 \%$ in the proportion of patients $<60$ years. The in-hospital mortality increased from $33 \cdot 1 \%$ to $40 \cdot 6 \%$ in the general population, and for patients that underwent respiratory support (NIV: $24.8 \%$ vs. 28.6\%, IMV: $78.8 \%$ vs. $84.1 \%$ ). However, the proportion of mortality should be interpreted with caution since there is still a substantial number of ongoing admissions (Table 7.1).

Based on the available data of 1,217,332 COVID-19 adult hospitalizations in Brazil, the second wave's progression resulted in an increased burden of severe cases similar to recently observed in the UK (CHALLEN et al., 2021), Africa (SALYER et al., 2021) and elsewhere. The Brazilian healthcare system, overwhelmed during the first wave, now indicates resource constraints, or even collapse, in a scenario of low adherence to non-pharmacological interventions and convergent dominance of the VoC. However, this work cannot stablish a causal relationship between VoC lineages and higher burden of severe cases or increased pathogenicity of the virus. These findings indicate the need for urgent actions to contain the transmission, expand the vaccination coverage, and improve the assistance of critical COVID-19 by providing access to health services and disseminating better available evidence. 

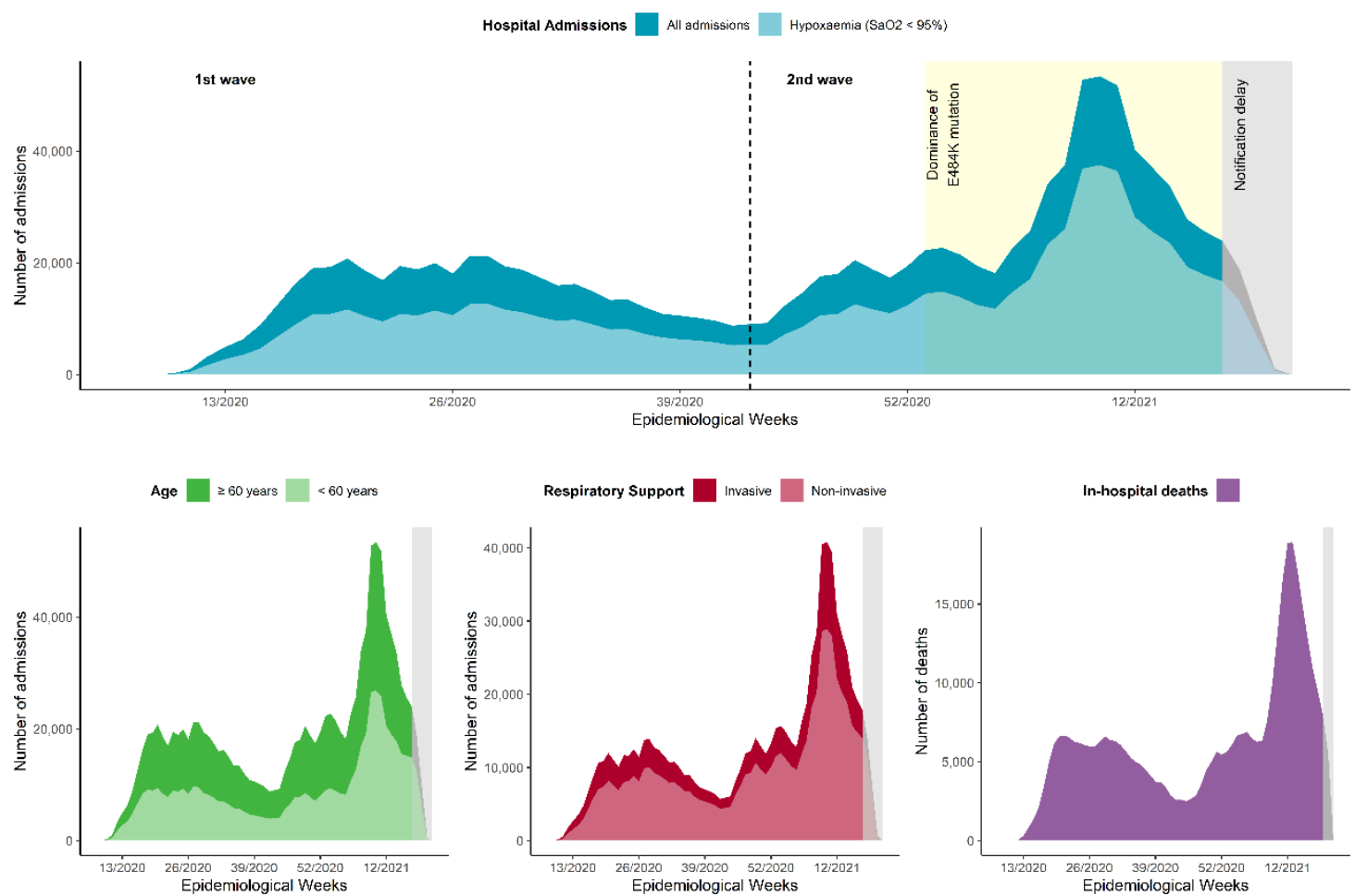

Figure 7.1 - Temporal increase in the number of COVID-19 hospital admissions and deaths in Brazil stratified by severity (hypoxaemia), age and respiratory support $(n=1,217,332$, all hospitalizations)

The $\mathrm{x}$-axis denotes the epidemiological week when symptom onset occurred for hospital admissions, and week of outcome for in-hospital deaths. First and second waves are defined by the lowest value per week of hospitalized cases in Brazil (dashed line, epidemiological week $43 / 2020$, October 18 to October 24,2020 ) whereas the yellow-shaded area starts after the E484K mutation's dominance (epidemiological week 53/2020, December 27, 2020 to January 02, 2021). The grey-shaded area represents a period of uncertainty, particularly for deaths, due to the expected notification delay from the SIVEP-Gripe (Data exported on May 24, 2021). 
Table 7.1 - Comparison of hospital admissions and in-hospital mortality between first and second COVID-19 waves in Brazil ( $n=1,187,840$ hospitalizations until May 01, 2021; 1,050,633 with a defined outcome)

\begin{tabular}{|c|c|c|c|c|}
\hline \multirow[b]{2}{*}{ Characteristics } & \multirow[b]{2}{*}{$\begin{array}{l}\text { First wave } \\
{[n=468,561]}\end{array}$} & \multirow[b]{2}{*}{$\begin{array}{l}\text { Second wave } \\
{[n=719,279]}\end{array}$} & \multicolumn{2}{|c|}{ Second wave ${ }^{\star \star}$} \\
\hline & & & $\begin{array}{c}\text { Before E484K } \\
\text { mutation } \\
\text { dominance } \\
{[n=170,718]}\end{array}$ & $\begin{array}{c}\text { After E484K } \\
\text { mutation } \\
\text { dominance } \\
{[\mathrm{n}=548,561]}\end{array}$ \\
\hline Admissions per week, median (IQR) & $\begin{array}{c}14,220 \\
(9,041-18,792)\end{array}$ & $\begin{array}{c}22,703 \\
(18,533-33,914)\end{array}$ & $\begin{array}{c}17,838 \\
(15392-19,331)\end{array}$ & $\begin{array}{c}27,791 \\
(22,732-37,556)\end{array}$ \\
\hline $\begin{array}{l}\text { Highest number of admissions in a } \\
\text { week }\end{array}$ & 21,294 & 53,424 & 22,319 & 53,424 \\
\hline Female, $n(\%)[n=1,187,650]$ & 205,555 (43.9\%) & 321,797 (44.7\%) & $76,025(44 \cdot 5 \%)$ & $245,772(44 \cdot 8 \%)$ \\
\hline $\begin{array}{l}\text { Age (years), median (IQR) [n = } \\
1,187,840]\end{array}$ & $62(48,74)$ & $60(48,71)$ & $63(50,74)$ & $59(47,70)$ \\
\hline $20-39$ & $61,510(13 \cdot 1 \%)$ & $92,387(12 \cdot 8 \%)$ & $18,663(10 \cdot 9 \%)$ & $73,724(13 \cdot 4 \%)$ \\
\hline $40-59$ & $153,331(32 \cdot 7 \%)$ & $256,940(35 \cdot 7 \%)$ & $53,846(31.5 \%)$ & $203,094(37.0 \%)$ \\
\hline$>=60$ & $253,720(54 \cdot 1 \%)$ & $369,952(51.4 \%)$ & $98,209(57.5 \%)$ & $271,743(49 \cdot 5 \%)$ \\
\hline $\begin{array}{l}\text { Residing in State capitals, } n(\%) \text { [n = } \\
1,187,840]\end{array}$ & $226,026(48 \cdot 2 \%)$ & $269,881(37 \cdot 5 \%)$ & $71,277(41 \cdot 8 \%)$ & $198,604(36 \cdot 2 \%)$ \\
\hline $\begin{array}{l}\text { Hypoxaemia, } n(\%)[n=1,005,396] \\
\text { ICU admission, } n(\%) \text { [n = 1,060,462] }\end{array}$ & $\begin{array}{l}273,071(69 \cdot 5 \%) \\
156,747(37 \cdot 6 \%)\end{array}$ & $\begin{array}{l}481,971(78 \cdot 7 \%) \\
241,371(37 \cdot 5 \%)\end{array}$ & $\begin{array}{l}105,168(72.9 \%) \\
59,806(38 \cdot 6 \%)\end{array}$ & $\begin{array}{l}376,803(80 \cdot 5 \%) \\
181,565(37 \cdot 1 \%)\end{array}$ \\
\hline $\begin{array}{l}\text { Respiratory Support, } \mathrm{n}(\%)[\mathrm{n}= \\
1,027,116]\end{array}$ & $291,463(73 \cdot 2 \%)$ & $524,788(83 \cdot 4 \%)$ & $115,693(77 \cdot 6 \%)$ & $409,095(85 \cdot 2 \%)$ \\
\hline NIV, $n(\%)[n=1,027,116]$ & $207,526(52 \cdot 1 \%)$ & $386,160(61.4 \%)$ & $87,939(59 \cdot 0 \%)$ & $298,221(62 \cdot 1 \%)$ \\
\hline IMV, $n(\%)[n=1,027,116]$ & $83,937(21 \cdot 1 \%)$ & $138,628(22 \cdot 0 \%)$ & $27,754(18.6 \%)$ & $110,874(23 \cdot 1 \%)$ \\
\hline IMV inside ICU, $n(\%)[n=217,376]$ & $70,764(86 \cdot 5 \%)$ & 116,457 (85.9\%) & $23,925(87.9 \%)$ & $92,532(85.4 \%)$ \\
\hline $\begin{array}{l}\text { Admissions with an outcome, } n(\%) \text { [n } \\
=1,187,840]\end{array}$ & $436,653(93 \cdot 2 \%)$ & $613,980(85 \cdot 4 \%)$ & $154,088(90 \cdot 3 \%)$ & $459,892(83 \cdot 8 \%)$ \\
\hline $\begin{array}{l}\text { In-hospital mortality }{ }^{*}, \mathrm{n}(\%)[\mathrm{n}= \\
1,050,633]\end{array}$ & $155,644(35 \cdot 6 \%)$ & $237,767(38 \cdot 7 \%)$ & $50,960(33 \cdot 1 \%)$ & $186,807(40 \cdot 6 \%)$ \\
\hline $20-39$ years $[n=132,946]$ & $6,547(11 \cdot 6 \%)$ & & $1,865(11 \cdot 2 \%)$ & $11,088(18 \cdot 5 \%)$ \\
\hline $40-59$ years $[n=356,306]$ & $30,924(21 \cdot 8 \%)$ & $58,824(27 \cdot 5 \%)$ & $9,193(19 \cdot 1 \%)$ & $49,631(29.9 \%)$ \\
\hline$>=60$ years $[n=561,381]$ & $118,173(49 \cdot 6 \%)$ & $165,990(51.4 \%)$ & $39,902(44 \cdot 7 \%)$ & $126,088(53.9 \%)$ \\
\hline ICU admission, $n(\%)[n=361,842]$ & $85,818(57 \cdot 8 \%)$ & $138,052(64 \cdot 7 \%)$ & $30,713(56 \cdot 0 \%)$ & $107,339(67 \cdot 7 \%)$ \\
\hline NIV,$n(\%)[n=518,072]$ & $52,014(26 \cdot 9 \%)$ & $89,796(27 \cdot 7 \%)$ & $19,604(24 \cdot 8 \%)$ & $70,192(28 \cdot 6 \%)$ \\
\hline $\operatorname{IMV}, \mathrm{n}(\%)[\mathrm{n}=208,560]$ & $64,260(79.2 \%)$ & $105,785(83.0 \%)$ & $20,823(78 \cdot 8 \%)$ & $84,962(84 \cdot 1 \%)$ \\
\hline
\end{tabular}

ICU - intensive care unit; NIV - Non-invasive ventilation; IMV - Invasive Mechanical Ventilation

*All in-hospital mortality estimates were calculated using only admissions with an outcome.

First wave - Epidemiological weeks 8/2020 to 43/2020 (February 16, 2020 to October 24, 2020)

Second wave - Epidemiological weeks 44/2020 to 17/2021* (October 25, 2020 to May 01, 2021)

${ }^{* *}$ We included data until week 17/2021 (May 01, 2021) to reduce potential effects from the notification delay on estimates.

Before E484K mutation dominance - Epidemiological weeks 44/2020 to 53/2020 (October 25, 2020 to January 02, 2021)

After E484K mutation dominance - Epidemiological weeks 01/2021 to 17/2021 


\title{
8 \\ Article 6 - Evolving changes in mortality of 13,301 critically ill adult patients with COVID-19 over eight months
}

\author{
This article was published at Intensive Care Medicine
}

\begin{abstract}
Purpose: Clinical characteristics and management of COVID-19 patients have evolved during the pandemic, potentially changing their outcomes. We analyzed the associations of changes in mortality rates with clinical profiles and respiratory support strategies in COVID-19 critically ill patients.

Methods: A multicenter cohort of RT-PCR-confirmed COVID-19 patients admitted at 126 Brazilian intensive care units between February $27^{\text {th }}$ and October $28^{\text {th }}, 2020$. Assessing temporal changes in deaths, we identified distinct time periods. We evaluated the association of characteristics and respiratory support strategies with 60-day inhospital mortality using random-effects multivariable Cox regression with inverse probability weighting.
\end{abstract}

Results: Of 13,301 confirmed-COVID-19 patients, 60-day in-hospital mortality was 13\%. Across four time periods identified, younger patients were progressively more common, noninvasive respiratory support was increasingly used, and the 60-day inhospital mortality has decreased in the last two periods. 4,188 patients received advanced respiratory support (noninvasive or invasive), from which $42 \%$ underwent only invasive mechanical ventilation, $37 \%$ only noninvasive respiratory support and $21 \%$ failed noninvasive support and were intubated. After adjusting for organ dysfunction scores and premorbid conditions, we found that younger age, absence of frailty and the use of noninvasive respiratory support (NIRS) as first support strategy were independently associated with improved survival (hazard ratio for NIRS first [95\% confidence interval], $0.59[0.54-0.65], \mathrm{p}<0.001)$.

Conclusion: Age and mortality rates have declined over the first eight months of the pandemic. The use of NIRS as the first respiratory support measure was associated with survival, but causal inference is limited by the observational nature of our data. 
Keywords: coronavirus; respiratory support; in-hospital mortality; respiratory support; noninvasive ventilation

\section{1 \\ Introduction}

Months after the Coronavirus Disease 19 (COVID-19) pandemic had spread across Asia and Europe, Brazil became a hotspot for the infection, with sustained transmission afterwards (TAYLOR, 2020; VAN DAMME et al., 2020). The general perception is that the proportion of severe cases declined as compared to the initial surge, with a younger population affected, resulting in lower case-fatality rates (BOEHMER et al., 2020). However, it is not clear how these changes have impacted the in-hospital outcomes of severe cases or how improvements in the clinical management of these patients may have led to the decline in mortality rates.

Although therapeutic options for severe COVID-19 patients have been tested recently in large clinical trials, (ANGUS et al., 2020; CAO et al., 2020; TOMAZINI et al., 2020; WANG et al., 2020) general questions on supportive care, such as the best initial ventilatory strategy, are still controversial. (FAN et al., 2020; WINCK; AMBROSINO, 2020) No conclusive data from randomized trials on SARS, MERS, or COVID-19 is currently available to guide ventilation practices. (ARABI; FOWLER; HAYDEN, 2020) Moreover, concerns related to the risk of aerosol generation and contamination of healthcare workers limited recommendations of noninvasive respiratory support strategies at the beginning of the COVID-19 epidemic (ORGANIZATION, 2020).

Resource limitations to treat severe COVID-19 patients have been a concern for international health authorities, societies, and researchers. (DONDORP et al., 2020; SOPEYIN et al., 2020) Recently, we demonstrated the impact on clinical outcomes of the collapse of health systems during the COVID-19 epidemic in Brazilian regions, especially for patients requiring mechanical ventilation (RANZANI et al., 2021; SALLUH; LISBOA; BOZZA, 2020). It is crucial to understand the clinical outcomes and factors contributing to mortality in different healthcare settings and, throughout the epidemic, to envision potential care improvement targets and optimal utilization of resources.

The present study analyzes the dynamic of severe COVID-19 admissions in 126 intensive care units (ICUs) from a middle-income country during the first eight months of the epidemic. We hypothesized that variations in clinical characteristics, risk factors 
and resource use were related to the evolving changes in mortality. In a secondary hypothesis, we evaluated the association of initial respiratory support strategies with 60day in-hospital mortality in patients with acute respiratory failure.

\section{2 \\ Patients and Methods}

\subsection{1}

\section{Study design and participants}

As the first confirmed SARS-CoV-2 infection in Brazil occurred on February $26^{\text {th }}$, this cohort study included patients admitted from February $27^{\text {th }}$ to October $28^{\text {th }}, 2020$, with vital status follow-up until December $27^{\text {th }}$. We included all adult patients with RTPCR-confirmed SARS-CoV-2 infection admitted to the ICUs from an integrated hospital network (Rede D'Or São Luiz) present in eight Brazilian States. All patients analyzed had COVID-19 as their primary ICU admission diagnoses. One hundred and twenty-six ICUs from 42 hospitals prospectively collected data on every consecutive ICU admission (Appendix A5.2). Local Ethics Committee and the Brazilian National Ethics Committee (CAAE: 17079119.7.0000.5249) approved the study without the need for informed consent.

\subsection{2}

\section{Data collection and missing values}

Anonymized information from COVID-19 ICU-admitted patients was obtained from an electronic system used for benchmarking purposes (Epimed Monitor ${ }^{\circledR}$, Rio de Janeiro, Brazil; ZAMPIERI et al., 2017) The database contains prospectively collected structured data of all ICU admissions. Characteristics at admission, including demographics, clinical diagnosis, comorbidities, source of admission, the Simplified Acute Physiology Score 3 (SAPS-3), the Sequential Organ Failure Assessment (SOFA) score, and the Modified Frailty Index (MFI), were considered for the analysis. We assessed the subsequent use of organ support, especially the initial advanced respiratory support implemented (noninvasive respiratory support strategies and invasive mechanical ventilation, NIRS and IMV, respectively), renal replacement therapy (RRT), vasopressors, and the hospital and ICU outcomes. NIRS was defined as either noninvasive positive pressure ventilation (NPPV) or high-flow nasal cannula (HFNC). Preparedness measures to absorb the surge of COVID-19-patients included the cancellation of elective surgeries, an increase in the number of ICU beds, and the 
implementation of care pathways for those with respiratory failure. To evaluate ICU preparedness, we compared the average number of ICU beds and occupancy rates between a pre-pandemic period (October/2019 to January/2020) and the peak of simultaneous hospitalizations.

We did not perform value imputation for the primary analysis, and we reported the number of complete cases for each variable (Appendix A6.1).

\subsubsection{Outcomes}

The primary outcome was 60-day in-hospital mortality. Secondary outcomes were in-hospital and ICU mortality, as well as hospital and ICU length-of-stays (LOS).

\subsection{4}

\section{Statistical Analysis}

We used median and interquartile range (IQR) or mean and standard deviation (SD) for quantitative variables, and frequencies and proportions for categories. We assessed the temporal dynamic of ICU hospitalizations and respiratory support utilization. We stratified our study population in time periods based on the daily number of ICU deaths using a method for evaluating structural changes in time series (e.g., inflection-point or change in trend; Appendix A6.1). Using linear models, this method identifies "breakpoints" in which a significant change in the curve's behavior (e.g., inflection-point or change in trend) occurred (ZEILEIS et al., 2002).

We compared clinical characteristics, organ support, and the use of NIRS or IMV as the first respiratory support measure, across the defined time periods. Amongst the subset of patients that required advanced respiratory support (NIRS and/or IMV), we performed univariate analyses of 60-day in hospital mortality using Kaplan-Meier (KM) survival curves. We considered age (categorized in decades with $<40$ years as the reference), frailty (categorized as non-frail, pre-frail and frail based on the MFI), and the initial respiratory support (NIRS first and IMV first), as the variables of clinical relevance, along with the time periods previously estimated. Differences among survival curves were evaluated with the log-rank test (confidence level: 0.05).

We evaluated the associations between the previously described variables with the 60-day in-hospital mortality in the subset of patients that required advanced respiratory support. We used a random-effects multivariable Cox proportional hazards model where the hazard is death. Due to the different case-mix among the hospitals, we considered the hospital variable as a source of random variability (random intercept) and adjusted the 
variables by the identified time periods. We estimated the Hazard Ratio (HR) and its corresponding $95 \%$ confidence interval for each variable. To account for the nonrandomized allocation of respiratory support strategies, we used propensity-scorederived inverse-probability treatment weighting (IPTW) in the multivariable Cox model. Propensity scores were estimated using multivariable logistic regression model with the first respiratory support as the response variable (GREIFER, 2020; GUO; FRASER, 2010; Appendix A6.1). Starting from a full multivariable model, we used a backward elimination process using $\mathrm{P}$-values in combination with goodness-of-fit measures (Akaike information criteria [AIC] and Bayesian information criteria [BIC]) to estimate the final model.

We also performed two sensitivity analyses to evaluate the robustness of our results. First, we built the final models using two alternative propensity-score-based methods: the Standardized Mortality Ratio (SMR)-weighting and IPTW excluding patients with propensity scores outside of the $95 \%$ percentile (KURTH et al., 2006). Second, we performed the multivariable model including only patients with available data on $\mathrm{PaO} 2 / \mathrm{FiO} 2$ ratio (with adjustment for this variable) and reported another model estimating missing values of lung injury severity with multiple imputation using chained equations (BUUREN; GROOTHUIS-OUDSHOORN, 2011).

We performed all analyses in R 4.0.2 (more details in Appendix A6.1).

\section{3 \\ Results}

From February $27^{\text {th }}$, to October $28^{\text {th }}, 2020$, a total of 61,471 consecutive adult ICU admissions occurred in 42 hospitals and 126 ICUs that prospectively collected data. From those, 13,301 (22\%) were patients with confirmed COVID-19 diagnosis, from which $4,188(31 \%)$ had respiratory failure requiring advanced respiratory support (NIRS or IMV) (Appendix A5.4). The overall median age was 54 years old (IQR:[41, 69]), with $39 \%(5,250 / 13,301)$ being 60 years or older and $42 \%$ women (Table 8.1). $13 \%$ of patients were frail $(\mathrm{MFI} \geq 3)$, and $68 \%$ presented at least one comorbidity (Appendix A.5.4). Among patients with $\mathrm{PaO} 2 / \mathrm{FiO} 2$ ratio available, almost half $(2,112 / 4,649 ; 45 \%)$ presented moderate to severe lung injury $\left(\mathrm{PaO}_{2} / \mathrm{FiO}_{2}\right.$ ratio $\left.\leq 200\right)$. The median ICU and hospital LOS were 5 days (IQR:[2-10]) and 8 days (IQR:[5-10]), respectively. Overall, 60-day in-hospital mortality was $13 \%(1,785 / 13,301)$. 
Table 8.1 - Clinical characteristics and outcomes of 13,301 critically ill COVID-19 patients, total and by periods*.

\begin{tabular}{|c|c|c|c|c|c|}
\hline Characteristics & $\begin{array}{c}\text { Total } \\
{[n=13,301]}\end{array}$ & $\begin{array}{c}\text { Period 1 } \\
{[n=2,184]}\end{array}$ & $\begin{array}{c}\text { Period 2 } \\
{[n=3,536]}\end{array}$ & $\begin{array}{c}\text { Period 3 } \\
{[n=3,938]}\end{array}$ & $\begin{array}{c}\text { Period } 4 \\
{[n=3,643]}\end{array}$ \\
\hline Age, Median (IQR) & $54(41,69)$ & $55(43,70)$ & $57(43,73)$ & $51(40,66)$ & $53(41,67)$ \\
\hline$<40$ & $2832(21 \%)$ & $421(19 \%)$ & $642(18 \%)$ & $943(24 \%)$ & $826(23 \%)$ \\
\hline $40-49$ & $2636(20 \%)$ & $421(19 \%)$ & $639(18 \%)$ & $853(22 \%)$ & $723(20 \%)$ \\
\hline $50-59$ & $2583(19 \%)$ & $433(20 \%)$ & $665(19 \%)$ & $769(20 \%)$ & $716(20 \%)$ \\
\hline $60-69$ & $2088(16 \%)$ & $354(16 \%)$ & $541(15 \%)$ & $558(14 \%)$ & $635(17 \%)$ \\
\hline $70-79$ & $1502(11 \%)$ & $275(13 \%)$ & $443(13 \%)$ & $414(11 \%)$ & $370(10 \%)$ \\
\hline$\geq 80$ & $1660(12 \%)$ & $280(13 \%)$ & $606(17 \%)$ & $401(10 \%)$ & $373(10 \%)$ \\
\hline \multicolumn{6}{|l|}{ Sex, No. (\%) } \\
\hline Female & 5549 (42\%) & $861(39 \%)$ & $1482(42 \%)$ & $1674(43 \%)$ & $1532(42 \%)$ \\
\hline Male & $7752(58 \%)$ & $1323(61 \%)$ & $2054(58 \%)$ & $2264(57 \%)$ & $2111(58 \%)$ \\
\hline $\begin{array}{l}\text { Admissions from emergency department } \\
\text { Modified Frailty Index (MFI) }\end{array}$ & $10240(77 \%)$ & $1576(72 \%)$ & $2479(70 \%)$ & $3236(82 \%)$ & $2949(81 \%)$ \\
\hline Mean (SD) & $1.07(1.25)$ & $1.13(1.25)$ & $1.26(1.33)$ & $0.96(1.20)$ & $0.96(1.19)$ \\
\hline Median (IQR) & $1(0,2)$ & $1(0,2)$ & $1(0,2)$ & $1(0,2)$ & $1(0,2)$ \\
\hline Non-frail $(M F I=0)$ & $5860(44 \%)$ & $914(42 \%)$ & $1335(38 \%)$ & $1884(48 \%)$ & $1727(47 \%)$ \\
\hline Pre-frail (MFI = 1-2) & $5717(43 \%)$ & $954(44 \%)$ & $1605(45 \%)$ & $1620(41 \%)$ & $1538(42 \%)$ \\
\hline Frail $(\mathrm{MFI}>=3)$ & $1724(13 \%)$ & $316(14 \%)$ & $596(17 \%)$ & $434(11 \%)$ & $378(10 \%)$ \\
\hline SAPS-3, Median (IQR) & $42(37,50)$ & $43(37,52)$ & $44(39,54)$ & $41(37,48)$ & $42(38,49)$ \\
\hline SOFA, Median (IQR) & $0(0,2)$ & $1(0,3)$ & $1(0,3)$ & $0(0,2)$ & $0(0,2)$ \\
\hline $\mathrm{PaO2} / \mathrm{FiO} 2[\mathrm{n}=4,649]$ & $\begin{array}{c}221 \\
(108,357)\end{array}$ & $\begin{array}{c}202 \\
(102,314)\end{array}$ & $\begin{array}{c}217 \\
(110,352)\end{array}$ & $\begin{array}{c}239 \\
(110,414)\end{array}$ & $\begin{array}{c}224 \\
(110,357)\end{array}$ \\
\hline Normal $(>300)$ & $1580(34 \%)$ & $238(27 \%)$ & $475(32 \%)$ & $501(40 \%)$ & $366(35 \%)$ \\
\hline Mild (201-300) & $957(21 \%)$ & $200(23 \%)$ & $310(21 \%)$ & $217(17 \%)$ & $230(22 \%)$ \\
\hline Moderate (101-200) & $1015(22 \%)$ & $217(25 \%)$ & $352(24 \%)$ & $238(19 \%)$ & $208(20 \%)$ \\
\hline Severe $(\leq 100)$ & $1097(24 \%)$ & $216(25 \%)$ & $339(23 \%)$ & $293(23 \%)$ & $249(24 \%)$ \\
\hline Oxygen support, No. (\%) & $9113(69 \%)$ & $1457(67 \%)$ & $2272(64 \%)$ & $2880(73 \%)$ & $2504(69 \%)$ \\
\hline Advanced respiratory support, №. (\%) & $4188(31 \%)$ & $727(33 \%)$ & $1264(36 \%)$ & $1058(27 \%)$ & $1139(31 \%)$ \\
\hline Noninvasive respiratory support (NIRS) & $2423(18 \%)$ & $182(8.3 \%)$ & $567(16 \%)$ & $772(20 \%)$ & $902(25 \%)$ \\
\hline Only NPPV & 2061 (85\%) & 168 (92\%) & $519(92 \%)$ & $659(85 \%)$ & $715(79 \%)$ \\
\hline Only HFNC & $136(5.6 \%)$ & $8(4.4 \%)$ & $26(4.6 \%)$ & $48(6.2 \%)$ & $54(6.0 \%)$ \\
\hline Both & $226(9.3 \%)$ & $6(3.3 \%)$ & $22(3.9 \%)$ & $65(8.4 \%)$ & $133(15 \%)$ \\
\hline Only NIRS & $1558(12 \%)$ & $84(3.8 \%)$ & $308(8.7 \%)$ & $513(13 \%)$ & $653(18 \%)$ \\
\hline NIRS failure & $865(6.5 \%)$ & $98(4.5 \%)$ & $259(7.3 \%)$ & $259(6.6 \%)$ & $249(6.8 \%)$ \\
\hline Only invasive mechanical ventilation (IMV) & $1765(13 \%)$ & $545(25 \%)$ & $697(20 \%)$ & $286(7.3 \%)$ & $237(6.5 \%)$ \\
\hline Vasopressor, No. (\%) & $1986(15 \%)$ & $476(22 \%)$ & $735(21 \%)$ & $402(10 \%)$ & $373(10 \%)$ \\
\hline Renal Replacement Therapy, No. (\%) & $989(7.4 \%)$ & $256(12 \%)$ & $367(10 \%)$ & $215(5.5 \%)$ & $151(4.1 \%)$ \\
\hline \multicolumn{6}{|l|}{ Length-of-stay (LOS), Median (IQR) } \\
\hline ICU $[n=13,294]$ & $5(2,10)$ & $6(3,13)$ & $6(3,12)$ & $4(2,9)$ & $5(2,9)$ \\
\hline Hospital $[n=13,219]$ & $8(5,15)$ & $9(6,18)$ & $10(6,18)$ & $7(5,14)$ & $7(5,13)$ \\
\hline \multicolumn{6}{|l|}{ Hospitalizations with LOS $>7$ days } \\
\hline ICU $[n=13,294]$ & $4660(35 \%)$ & $899(41 \%)$ & $1398(40 \%)$ & $1190(30 \%)$ & $1173(32 \%)$ \\
\hline Hospital $[n=13,219]$ & $7304(55 \%)$ & $1324(61 \%)$ & $2252(64 \%)$ & $1931(49 \%)$ & $1797(50 \%)$ \\
\hline 60-day in-hospital deaths, No. (\%) & $1785(13 \%)$ & $380(17 \%)$ & $649(18 \%)$ & $405(10 \%)$ & $351(9.6 \%)$ \\
\hline Only NIRS $[n=1,558]$ & $72(4.6 \%)$ & $6(7.1 \%)$ & $14(4.5 \%)$ & $22(4.3 \%)$ & $30(4.6 \%)$ \\
\hline NIRS failure [ $n=865$ ] & $444(51 \%)$ & $40(41 \%)$ & $125(48 \%)$ & $139(54 \%)$ & $140(56 \%)$ \\
\hline Only IMV $[n=1,765]$ & $1028(58 \%)$ & $285(52 \%)$ & $430(62 \%)$ & $177(62 \%)$ & $136(57 \%)$ \\
\hline ICU deaths, No. (\%) [n = 13,294] & $1446(11 \%)$ & $321(15 \%)$ & $542(15 \%)$ & $317(8.1 \%)$ & $266(7.3 \%)$ \\
\hline In-hospital deaths, No. (\%) $[n=13,219]$ & $1814(14 \%)$ & $385(18 \%)$ & $662(19 \%)$ & $412(11 \%)$ & $355(9.8 \%)$ \\
\hline Ongoing patients, No. (\%) & $82(0.6 \%)$ & $16(0.7 \%)$ & $26(0.7 \%)$ & $15(0.4 \%)$ & $25(0.7 \%)$ \\
\hline
\end{tabular}

${ }^{*}$ Period $1-$ February $27^{\text {th }}$ to April $25^{\text {th }} ;$ Period $2-$ April $26^{\text {th }}$ to June $6^{\text {th }} ;$ Period $3-$ June $7^{\text {th }}$ to August $10^{\text {th }} ;$ Period $4-$ August $11^{\text {th }}$ to October $28^{\text {th }}$

SD - Standard deviation; IQR - Interquartile Range; SAPS - Simplified Acute Physiology Score; SOFA - Sequential Organ Failure Assessment; NIRS - Noninvasive Respiratory Support; NPPV - Noninvasive Positive Pressure Ventilation; HFNC - High-Flow Nasal Cannula; IMV - Invasive Mechanical Ventilation; ICU - Intensive care unit

Over time, since the first confirmed cases in late February and early March, a sharp rise in daily ICU admissions for COVID-19 was observed, with a subsequent increase in daily deaths (Figure 8.1). This upward trend remained until May $13^{\text {th }}, 2020$, with a peak 
of 1,066 ICU-hospitalized patients in a single day. ICU mortality rate peaked $34 \%$ on May $24^{\text {th }}$. In comparison with the pre-pandemic period (October/2019-January/2020), ICU bed availability increased $31 \%$ at the peak of ICU hospitalizations (Appendix A6.4).
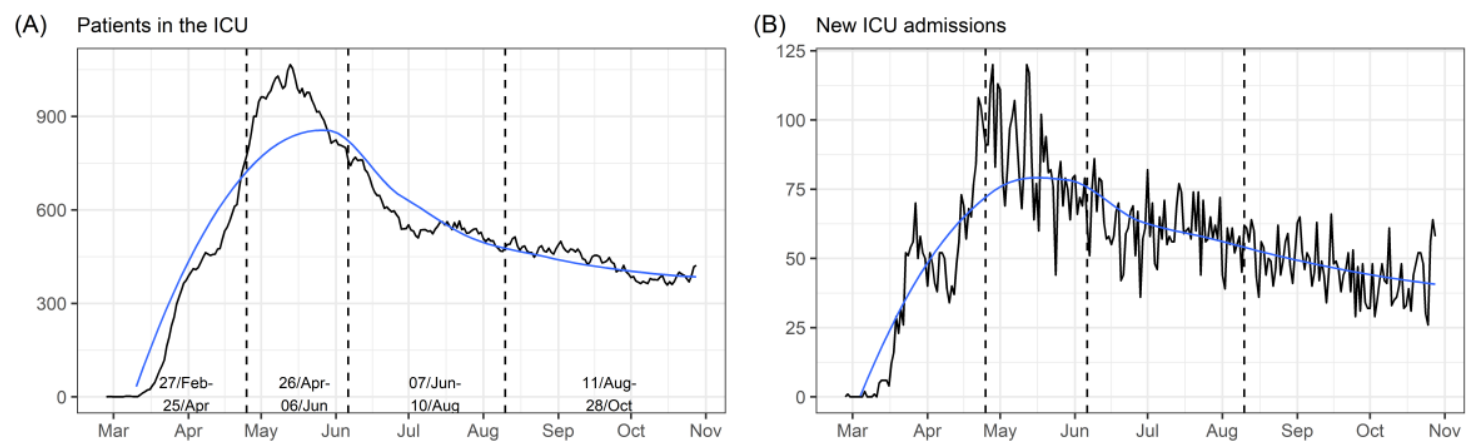

(C) ICU deaths

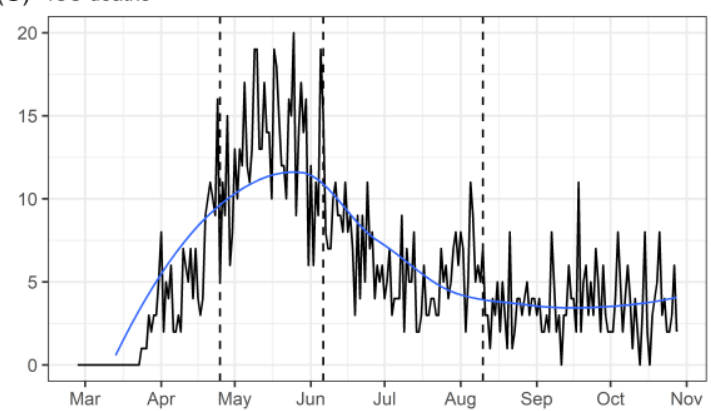

(D) In-hospital mortality rate

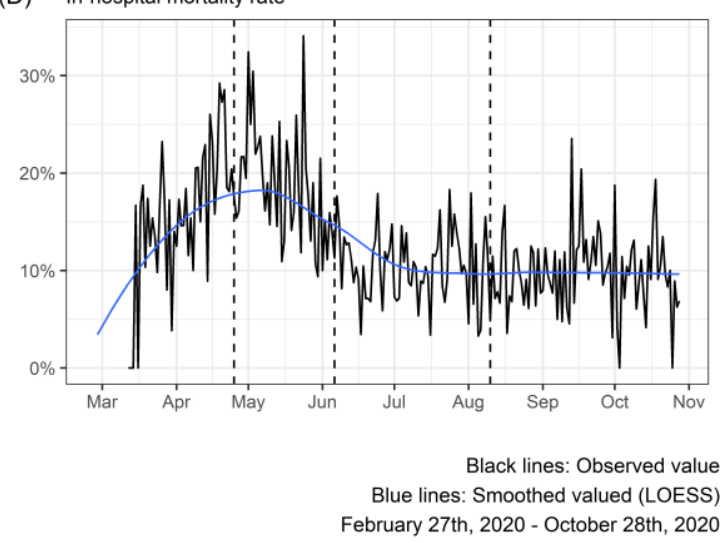

Figure 8.1 - Progression of adult ICU admissions with COVID-19 from February 27th, 2020 to October 28th, 2020

(A) total patients in the ICU per day; (B) the number of new ICU admissions per day; (C) the number of deaths in the ICU per day; (D) the daily mortality rate in the ICU (using the admission date as the reference). The black line represents daily absolute numbers, and the blue line is the smoothed curve. The three dashed lines correspond to the estimated breakpoints of structure change in the time series of ICU deaths rate panel (C): April $25^{\text {th }}$, June $06^{\text {th }}$, and August $10^{\text {th }}$, respectively.

Based on the analysis of structural changes in the time series of the daily ICU deaths curve (Figure 8.1, panel C), three breakpoints were identified, and four time periods were defined to stratify our population. Patient's characteristics and outcomes per period are described in Table 1. 60-day in-hospital mortality rates were: Period 1, 17\%; Period 2, 18\%, Period 3, 10\%; Period 4, 9.6\%. Patients in Periods 1 and 2 were older and more frequently frail. Clinical severity was highest in Period 2 (median SAPS-3, 44 IQR: [39, 54]), while the need for vasopressors and RRT were worse in period 1 (22\% and $12 \%$, respectively). Regarding modes of advanced respiratory support, we observed a 
progressive increase in the use of NIRS across the four time periods (Period 1, 8.3\%; Period 2, 16\%, Period 3, 20\%, and Period 4, 25\%).

(A)
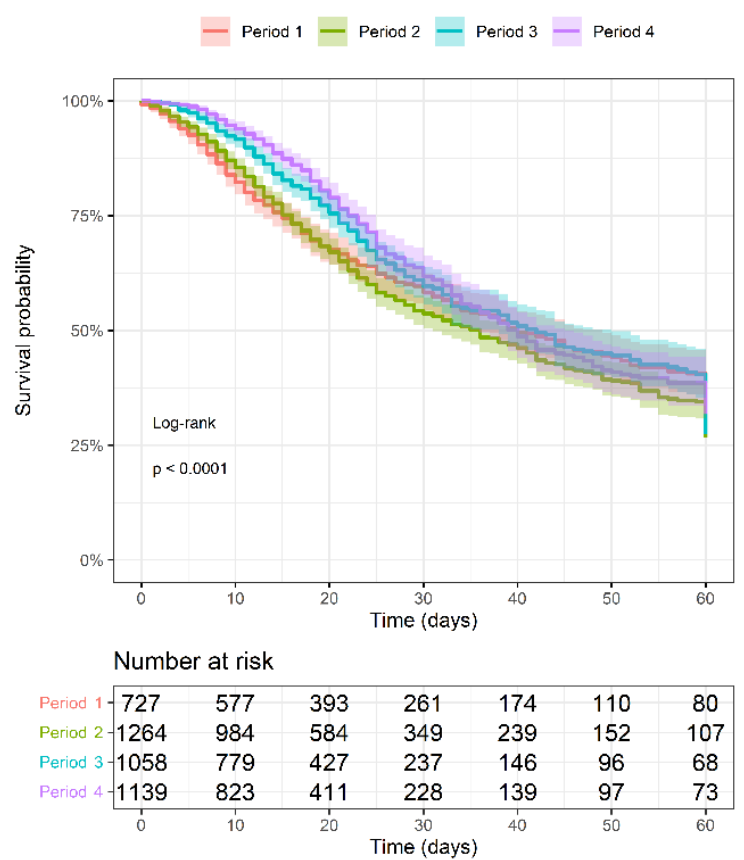

(C)

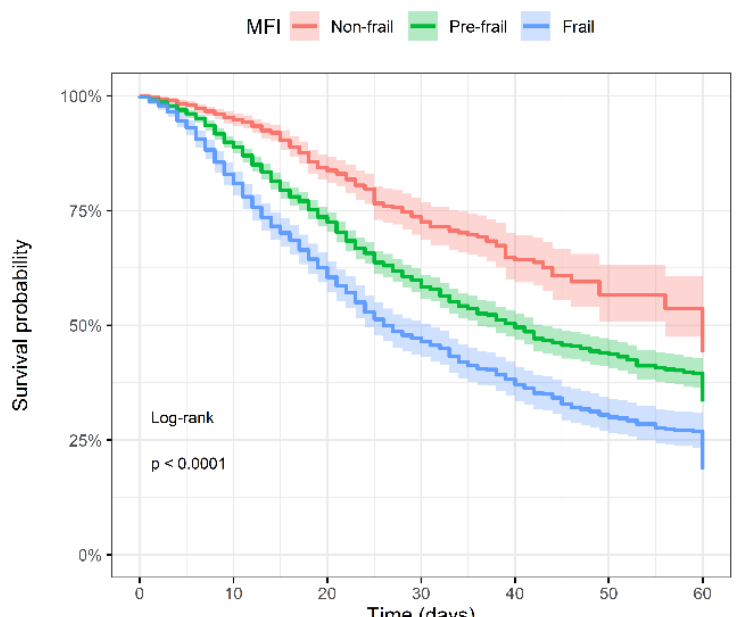

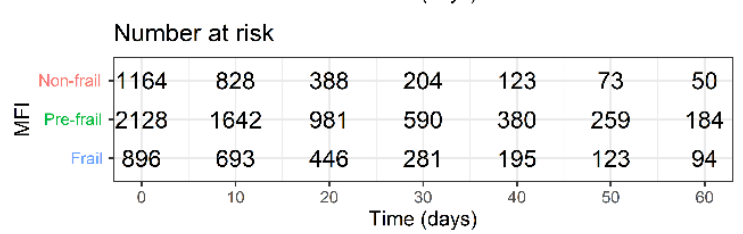

(B)

Age $-<40-40-49=50-59-60-69-70-79-280$
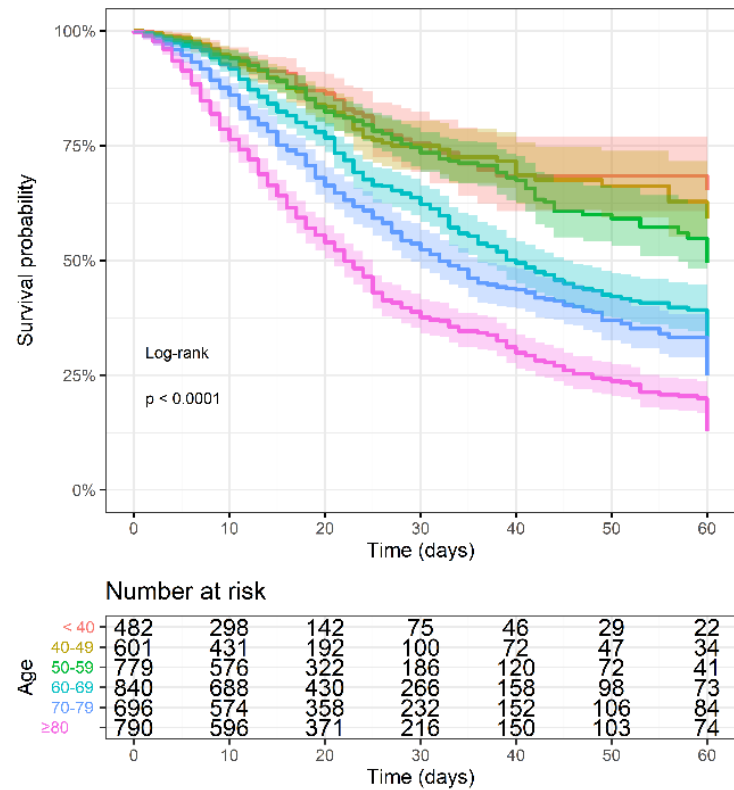

(D)

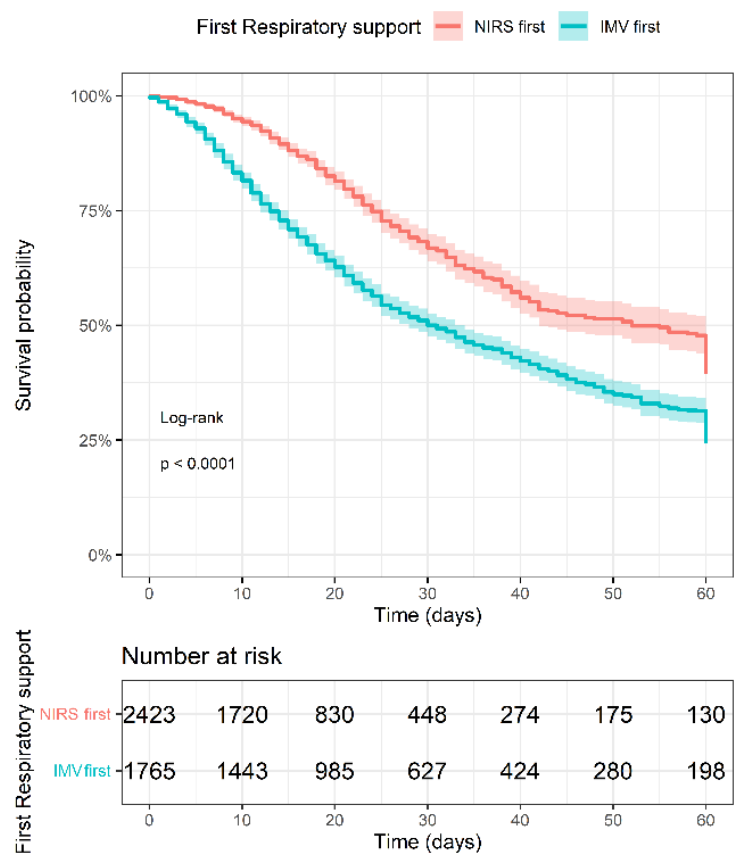

Figure 8.2 - Univariable survival curves (Kaplan-Meier) of factors related to the 60-day outcome in critically ill patients that underwent advanced respiratory support

(A) Time periods estimated with the breakpoints of structure change (Period 1: February $27^{\text {th }}$ to April 25 ${ }^{\text {th }}$; Period: April 26th to June $6^{\text {th }}$; Period 3: June $7^{\text {th }}$ to August $10^{\text {th }}$; Period 4: August 11 th to October 28 ${ }^{\text {th }}$ ); (B) Age (<40, 40-49, 50-59, 60-69, 70-79, $\geq 80$; (C) Modified Frailty Index (MFI) at the admission, with groups Non-frail (MFI =0), Pre-frail (MFI = 1-2) and Frail (MFI $\geq 3)$; (D) Initial respiratory support considering noninvasive (NIRS first) invasive (IMV first). Differences among curves were assessed using the log-rank test with a confidence level of 0.05 . 
Respiratory failure requiring advanced respiratory support occurred in 4,188 out of 13,301 (31\%) patients (Appendix A6.4). Of these, 1,765 (42\%) underwent invasive mechanical ventilation. In patients that received NIRS $(\mathrm{N}=2,423), 2,061(85 \%)$ used NPPV, 136 (6\%) HFNC and 226 (9\%) received both treatment modes. 60-day in-hospital mortality was higher in those that underwent only IMV or failed NIRS as compared to those that required only NIRS (58 or 51\% vs. 4.6\%; Table 8.2). In patients that underwent advanced respiratory support, the probability of survival was lowest in Periods 1 and 2 (Log-rank p<0.0001, Figure 8.2, panel A). Stratification by age and frailty revealed progressively worse survival probabilities in patients older than 60 years old and those that were pre-frail or frail, respectively (Log-rank p $<0.001$ in both, Figure 8.2, panels B and C). Regarding the initial strategies of respiratory support, the best survival probabilities were among patients that used NIRS as the first respiratory support measure as opposed to patients that were initially intubated (Figure 8.2 panel D). Even patients with NIRS failure and subsequent intubation showed better survival probabilities compared to patients that first received IMV (Appendix A6.4).

We evaluated the association of clinical characteristics, risk factors and initial respiratory support strategies with 60-day in-hospital mortality in the subset of patients that underwent advanced respiratory support. We estimated a random-effects multivariable Cox model with IPTW (Figure 8.3; Appendix A5.5). We found that older age (60-69 years, HR [95\% CI]: 1.47 [1.20-1.80], p<0.001; 70-79 years, HR [95\% CI]: 1.71 [1.38-2.10], $\mathrm{p}<0.001 ; \geq 80$ years, HR [95\% CI]: 2.75 [2.21-3.41], $\mathrm{p}<0.001)$ and the presence of frailty $(\mathrm{MFI} \geq 3, \mathrm{HR}$ [95\% CI]: 1.38 [1.15-1.64], p<0.001) were independently associated with worse 60-day survival. Moreover, the use of NIRS, as the first respiratory support, was associated with improved survival over 60 days (HR [95\% CI]: 0.59 [0.540.65], $\mathrm{p}<0.001$ ), after adjusting for the time periods, age, gender, frailty, SAPS-3 and SOFA scores, comorbidities, and source of admission (Figure 8.3; Appendix A6.5). No significant multicollinearity was detected in the final model. All performed sensitivity analyses and alternative models demonstrated similar results in comparison with our primary analysis (Appendix A6.6). 
Table 8.2 - Characteristics and outcomes of critically ill patients stratified by advanced respiratory support

\begin{tabular}{|c|c|c|c|c|}
\hline Characteristics & $\begin{array}{c}\text { Total } \\
{[n=4,188]}\end{array}$ & $\begin{array}{l}\text { NIRS only } \\
{[n=1,558]}\end{array}$ & $\begin{array}{l}\text { NIRS failure } \\
{[n=865]}\end{array}$ & $\begin{array}{c}\text { IMV } \\
{[n=1,765]}\end{array}$ \\
\hline Age, Median (IQR) & $63(49,76)$ & $55(43,67)$ & $65(53,77)$ & $68(54,80)$ \\
\hline$<40$ & $482(12 \%)$ & $286(18 \%)$ & $65(7.5 \%)$ & $131(7.4 \%)$ \\
\hline $40-49$ & $601(14 \%)$ & $307(20 \%)$ & $105(12 \%)$ & $189(11 \%)$ \\
\hline $50-59$ & $779(19 \%)$ & $376(24 \%)$ & $143(17 \%)$ & $260(15 \%)$ \\
\hline $60-69$ & $840(20 \%)$ & $270(17 \%)$ & 227 (26\%) & $343(19 \%)$ \\
\hline $70-79$ & $696(17 \%)$ & $162(10 \%)$ & $143(17 \%)$ & $391(22 \%)$ \\
\hline$\geq 80$ & $790(19 \%)$ & $157(10 \%)$ & $182(21 \%)$ & $451(26 \%)$ \\
\hline \multicolumn{5}{|l|}{ Sex, No. (\%) } \\
\hline Female & $1516(36 \%)$ & $546(35 \%)$ & $305(35 \%)$ & $665(38 \%)$ \\
\hline Male & $2672(64 \%)$ & $1012(65 \%)$ & $560(65 \%)$ & $1100(62 \%)$ \\
\hline $\begin{array}{l}\text { Admissions from emergency department } \\
\text { Modified Frailty Index (MFI) }\end{array}$ & $2848(68 \%)$ & $1244(80 \%)$ & $581(67 \%)$ & $1023(58 \%)$ \\
\hline Non-frail $(M F I=0)$ & $1164(28 \%)$ & $617(40 \%)$ & $199(23 \%)$ & $348(20 \%)$ \\
\hline Pre-frail (MFI = 1-2) & $2128(51 \%)$ & $732(47 \%)$ & $459(53 \%)$ & $937(53 \%)$ \\
\hline Frail $(\mathrm{MFI}>=3)$ & $896(21 \%)$ & $209(13 \%)$ & 207 (24\%) & $480(27 \%)$ \\
\hline SAPS-3, Median (IQR) & $50(42,61)$ & $43(39,51)$ & $54(45,66)$ & $55(46,67)$ \\
\hline$\leq 42$ & $1,165(28 \%)$ & $727(47 \%)$ & $147(17 \%)$ & $291(16 \%)$ \\
\hline $43-50$ & $982(23 \%)$ & $434(28 \%)$ & $198(23 \%)$ & $350(20 \%)$ \\
\hline $51-61$ & $1,034(25 \%)$ & $276(18 \%)$ & $242(28 \%)$ & $516(29 \%)$ \\
\hline$>61$ & $1,007(24 \%)$ & $121(7.8 \%)$ & $278(32 \%)$ & $608(34 \%)$ \\
\hline SOFA, Median (IQR) & $2(0,5)$ & $1(0,2)$ & $3(1,7)$ & $4(1,8)$ \\
\hline Any comorbidities, No. (\%) & $3393(81 \%)$ & $1111(71 \%)$ & $754(87 \%)$ & $1528(87 \%)$ \\
\hline $\mathrm{PaO2} / \mathrm{FiO2}[\mathrm{n}=1,963]$ & $170(94,279)$ & $216(89,329)$ & $142(90,233)$ & $172(101,273)$ \\
\hline Normal $(>300)$ & $431(22 \%)$ & $139(32 \%)$ & $75(15 \%)$ & $217(21 \%)$ \\
\hline Mild (201-300) & $385(20 \%)$ & $89(21 \%)$ & $91(18 \%)$ & $205(20 \%)$ \\
\hline Moderate $(101-200)$ & $621(32 \%)$ & $78(18 \%)$ & $191(38 \%)$ & $352(34 \%)$ \\
\hline Severe $(\leq 100)$ & $526(27 \%)$ & $125(29 \%)$ & $146(29 \%)$ & $255(25 \%)$ \\
\hline \multicolumn{5}{|l|}{ Noninvasive respiratory support } \\
\hline Only NPPV & $2061(85 \%)$ & $1356(87 \%)$ & $705(82 \%)$ & - \\
\hline Only HFNC & $136(5.6 \%)$ & $87(5.6 \%)$ & $49(5.7 \%)$ & - \\
\hline Both & $226(9.3 \%)$ & $115(7.4 \%)$ & $111(13 \%)$ & - \\
\hline Vasopressor, No. (\%) & $1890(45 \%)$ & $60(3.9 \%)$ & $672(78 \%)$ & $1158(66 \%)$ \\
\hline Renal Replacement Therapy, No. (\%) & $896(21 \%)$ & $24(1.5 \%)$ & $278(32 \%)$ & $594(34 \%)$ \\
\hline \multicolumn{5}{|l|}{ Length-of-stay (LOS), Median (IQR) } \\
\hline $\operatorname{ICU}[n=4,185]$ & $12(7,22)$ & $8(4,11)$ & $19(12,27)$ & $16(9,27)$ \\
\hline Hospital $[n=4,160]$ & $17(10,30)$ & $11(8,16)$ & $24(16,38)$ & $22(12,38)$ \\
\hline \multicolumn{5}{|l|}{ Hospitalizations with LOS $>7$ days } \\
\hline ICU $[n=4,185]$ & $3011(72 \%)$ & $788(51 \%)$ & $787(91 \%)$ & $1436(81 \%)$ \\
\hline Hospital $[n=4,160]$ & $3496(84 \%)$ & $1166(75 \%)$ & $804(94 \%)$ & $1526(87 \%)$ \\
\hline Period 1 (February 27th to April $25^{\text {th }}$ ) & $727(17 \%)$ & $84(5.4 \%)$ & $98(11 \%)$ & $545(31 \%)$ \\
\hline Period 2 (April 26th to June $6^{\text {th }}$ ) & $1264(30 \%)$ & $308(20 \%)$ & $259(30 \%)$ & $697(39 \%)$ \\
\hline Period 3 (June 7 th to August $10^{\text {th }}$ ) & $1058(25 \%)$ & $513(33 \%)$ & $259(30 \%)$ & $286(16 \%)$ \\
\hline Period 4 (August 11th to October $28^{\text {th }}$ ) & $1139(27 \%)$ & $653(42 \%)$ & $249(29 \%)$ & $237(13 \%)$ \\
\hline 60-day in-hospital deaths, No. (\%) & $1544(37 \%)$ & $72(4.6 \%)$ & $444(51 \%)$ & $1028(58 \%)$ \\
\hline ICU deaths, No. $(\%)[n=13,294]$ & $1329(32 \%)$ & $47(3.0 \%)$ & $398(46 \%)$ & $884(50 \%)$ \\
\hline In-hospital deaths, No. (\%) $[n=13,219]$ & $1572(38 \%)$ & $73(4.7 \%)$ & $457(53 \%)$ & $1042(59 \%)$ \\
\hline
\end{tabular}

SD - Standard deviation; IQR - Interquartile Range; SAPS - Simplified Acute Physiology Score; SOFA - Sequential Organ Failure Assessment; NIRS - Noninvasive Respiratory Support; NPPV - Noninvasive Positive Pressure Ventilation; HFNC - High-Flow Nasal Cannula; IMV - Invasive Mechanical Ventilation; ICU - Intensive care unit 


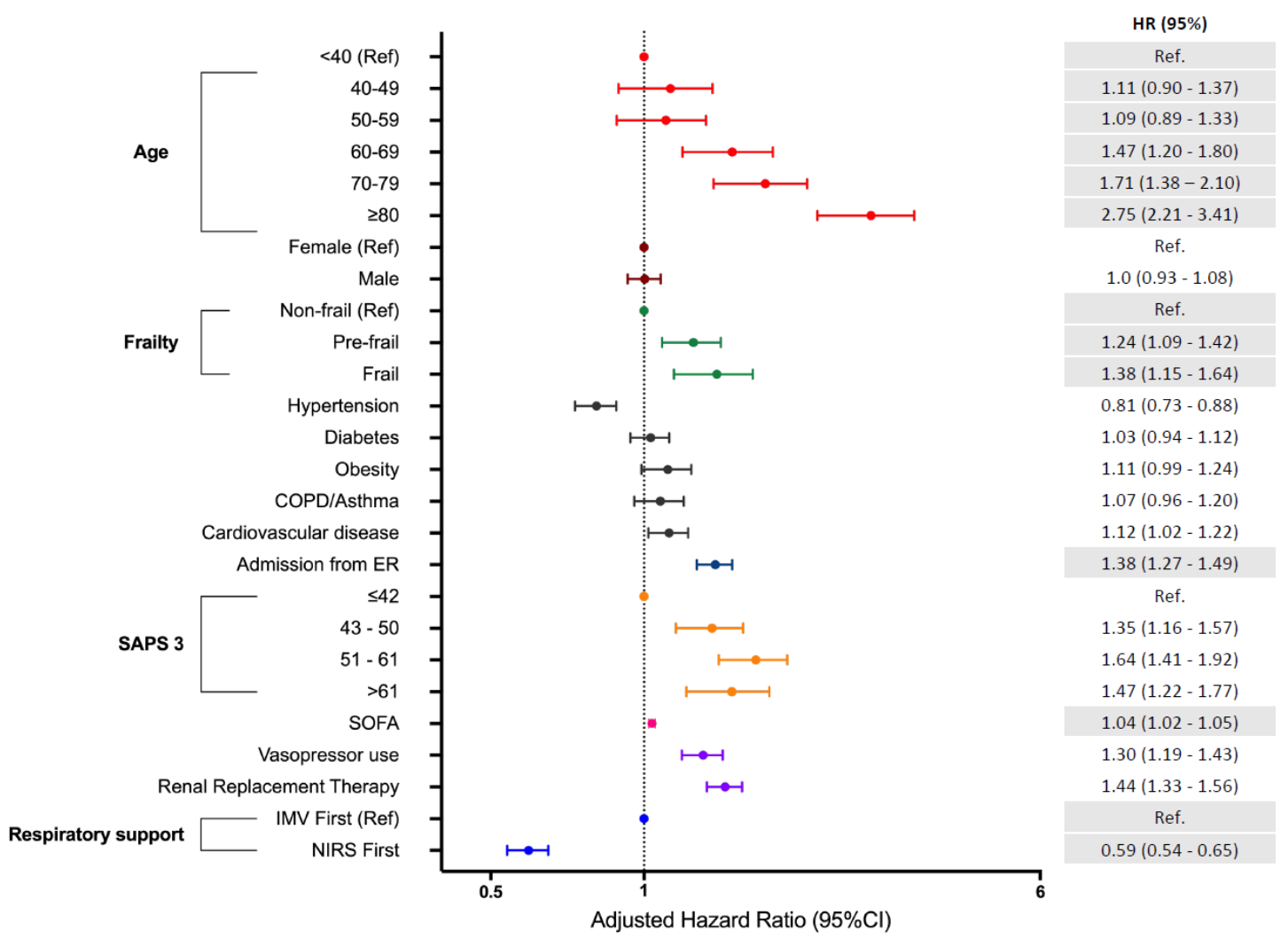

Figure 8.3 - Random-effects multivariable cox proportional hazards model to assess the association of clinical characteristics and initial respiratory support with 60-day mortality in patients that underwent advanced ventilatory support (NIRS and/or IMV), adjusted by the timeperiod of admission. The hospital was considered as the random intercept (Standard deviation = 0.50). To account for the nonrandomization, we used inverse-probability treatment weighting (IPTW) of propensity scores regarding the initial use of NIRS. We provide the Hazard Ratio (HR) for 60-day in-hospital mortality and its respective $95 \%$ confidence intervals for each variable.

\section{4 \\ Discussion}

In this large cohort of critically ill COVID-19 patients from South America, we showed the dynamic of the first eight months of the epidemic and the evolving changes in clinical characteristics, respiratory support practices, and hospital mortality rates. Age and mortality rates declined over time after the peak in hospitalizations occurred, while daily ICU admissions reached a plateau. We further identified clinical predictors of 60day in-hospital mortality, including increased age ( $>60$ years), the presence of frailty, multiple organ dysfunction, and the need for invasive mechanical ventilation. Finally, we observed an independent association between the increasing use of NIRS with improved survival in this population. 
The dynamic reported in this work corresponded to the expansion of the outbreak over eight months in the metropolitan areas of the country's southeast region $(\sim 70 \%$ of this sample). It also reflects a healthcare system that underwent preparedness with increases in ICU-bed and resource availability, which resulted in almost unrestricted access to ICU care. Our current findings contrast with recently published national data from the first 250 thousand patients hospitalized for COVID-19 in Brazil, where ICU mortality was 55\%. Even in the southeast region of the country - where the majority of our cohort was treated - authors showed that mortality in ICU patients was $49 \%$ and among those invasively ventilated it was 77\% (RANZANI et al., 2021). These differences may be due to early hospitalization, monitoring and good clinical practices performed in this hospital network.

We analyzed the clinical characteristics and outcomes of critically ill COVID-19 patients admitted to 126 ICUs in 42 hospitals. We present complete data on 60-day inhospital outcomes (in addition to $99 \%$ of patients with available hospital mortality) from patients admitted over eight months. To study the pandemic's temporal evolution, we analyzed structural changes in the mortality rate curve, which defined three breakpoints and four time periods. Survival was worst in the period when hospitalizations peaked, and patients were older and more frequently frail (Period 2). However, our results suggest that patients' characteristics alone do not explain the progressive reduction in mortality observed in periods 3 and 4. Changes in management, such as the increased use of NIRS, were also related to improved survival rates in the subset of more severe patients that required advanced respiratory support.

In a European cohort from 3 countries, (SCHMIDT et al., 2021) investigators from the COVID-ICU group showed an overall decrease in 90-day mortality over time in critically-ill patients from $42 \%$ in early March to $25 \%$ in late April 2020. Although they observed an increasing use of NIRS (mainly HFNC) and $41 \%$ of patients received steroids, the associations of these interventions with mortality were not analyzed. (SCHMIDT et al., 2021) Overall, the use of steroids increased over time in the hospitals analyzed (Appendix A6.6). However, since we did not have individual data on treatment, we cannot exclude that changes in clinical management and other unmeasured interventions may have affected our findings of improved survival over time (STERNE et al., 2020). Nevertheless, we clearly demonstrated that NIRS (mainly NPPV in our 
cohort) was increasingly used over time during the study period and that, after adjusting for characteristics and time periods, it was associated with better survival.

In our study, $31 \%$ of patients required advanced ventilatory support (NIRS or IMV), which is lower than other multicenter ICU cohorts as well as data from the Brazilian ICU Registry. (THERNEAU, TERRY M., [s.d.]) Also, ventilated patients in our cohort were younger (median: 63 vs. 73 and 71 years in the UK and German cohorts, respectively), nevertheless $81 \%$ presented comorbidities, and $21 \%$ were previously frail. Survival was progressively lower in patients older than 60 years and in those considered pre-frail or frail. We also observed a significantly higher NIRS utilization rate than other cohorts (Germany [KARAGIANNIDIS et al., 2020] 5\%, US [GUPTA et al., 2020] 1\%, Italy [GRASSELLI et al., 2020] <10\%). As expected, patients that underwent NIRS without subsequent intubation had improved survival as compared to those under invasive mechanical ventilation, but surprisingly, even patients that failed NIRS and were intubated also showed better survival compared to those that were intubated directly.

Concerns on biosafety aspects and potential intubation delays have limited the use of NIRS for respiratory failure early in the pandemic. However, NPPV and HFNC represent essential strategies in responding to respiratory emerging infections such as COVID-19, particularly in resource-limited settings, by optimizing critical care resources (i.e., invasive mechanical ventilation). In the absence of randomized trials, both from past severe viral infections and the current epidemic, our results that NIRS failure did not worsen mortality in comparison to those intubated directly, are reassuring for physicians using NIRS as an early option of ventilatory support for COVID-19. However, the potential benefit of noninvasive respiratory strategies in COVID-19-associated respiratory failure has yet to be determined by ongoing clinical trials (ISRCTN16912075/ PERKINS et al., 2020).

The strengths of our study consist in being one of the largest multicenter cohorts of ICU-hospitalized patients with COVID-19, showing evolving mortality reductions in those critically ill. All patients had 60-day outcomes and detailed baseline severity of illness, comorbidities, frailty, organ dysfunction, and resource use information. Furthermore, we evaluated the association of respiratory support, especially NIRS, with 60-day mortality, which can inform future clinical trials and clinical practices for ICU patients. Potential limitations include: first, our sample may not reflect the epidemiology and practices in COVID-19 patients admitted to most Brazilian ICUs. Nonetheless, we 
showed data from a large network of hospitals with optimal preparedness and resource availability. Second, we cannot exclude that changes in clinical management and other unmeasured interventions may have affected survival over time, such as steroids, anticoagulation, and others. However, our models were adjusted for several clinically important covariates, including the 4 time periods over 8 months. Third, we analyzed NPPV and HFNC as one combined group of NIRS. We did not have specific data on NPPV-delivery methods (face mask or helmet) and only a small minority of patients underwent HFNC. These limitations prevent the interpretation of our results for any specific noninvasive ventilation mode. Fourth, we did not have imaging data on lung infiltrates or the diagnosis of viral pneumonia. However, all patients included in this analysis had a primary ICU admission diagnosis of COVID-19 infection and required at least oxygen support in the ICU. Finally, although we had complete 60-day outcomes, the long-term follow-up and data on post-ICU quality of life or post-intensive care syndrome were unavailable.

\section{5}

\section{Conclusion}

In this large cohort of critically ill COVID-19 patients from South America, we demonstrated that, after a peak in hospitalizations occurred in May 2020, age and mortality rates have declined over the last five months of the epidemic. We also found an association between the use of noninvasive respiratory support and improved survival, even after accounting for age, frailty, organ failures, and conversion to invasive mechanical ventilation. These results, however, should be interpreted with caution, due to the observational nature of our data. 


\section{9 \\ Final Considerations}

Adequate management of healthcare resources provides better care for patients, especially under conditions of low-resource availability or constraints. In this thesis, we addressed resource evaluation under usual conditions and in high stress and strain settings. Under these two questions, we conducted six data science projects and used datadrive and statistical methods to analyze data and provide insightful information for decision-makers and policymakers.

We evaluated the management of resources in Brazilian and Dutch ICUs in the preCOVID-19 pandemic period using benchmarking methods. The analyses on two samples of ICUs showed that different organizational aspects were associated with increased efficiency and are potential targets for improvement. Although we focused on ICU data, this analysis could be extended to other healthcare departments, especially when riskadjusted metrics are possible to calculate.

In a technical analysis, we observed that performance metrics could be directly combined into a single indicator when their correlation is high, such as the dataset of Brazilian units. Although the decision on the combination of metrics depends on the objective, a continuous metrics is more favorable for ICU benchmarking since it offers better statistical properties for statistical modelling.

Under the context of the COVID-19 pandemic, although regular benchmarking methods could not be directly applied, we could evaluate the use of resources and outcomes during this period. We executed two extensive research studies to assess national data from the COVID-19 admissions. Our findings showed that in a first moment the pandemic had a regional and temporal impact, where regions with the most vulnerable systems presented high mortality levels and increased use of ICU resources. Those impacts were intensified in a second and larger surge of hospital admissions, under the context of more transmissible variants of concern and low adherence to nonpharmacological interventions.

Those studies comprised one of the first large reports of COVID-19 admissions in the country. Our results assisted the planning of pandemic mitigation and control actions 
in the Brazilian States, and we also developed an online dashboard to provide those analysis and insightful information for the public.

Although national data showed large mortality rates, we showed that the overall mortality rate was low under conditions of preparedness and wide availability of resources, even in a middle-income country. In these settings, we observed that mortality rates for ICU patients with COVID-19 decreased over time, with an increased use of noninvasive respiratory support, even though severity of illness decreased.

Our studies were mainly dependent on the data available for analysis. We used large datasets of patients with a considerable representation of the healthcare system. However, most of our results identify associations between variables of interest and outcomes. Hence, one should not make causality conclusions upon the information provided. Additional data should also be included and monitored since, for instance, the behavior of the healthcare system, outcomes, and resources changes over time and along with the strain/stress context.

Although extensive, the results obtained in each of the research studies can be explored in future research. We list a few potential research studies following the current findings:

- Benchmarking ICU (or hospitals) outcomes for COVID-19 patients: Applying traditional benchmarking methods in COVID-19 pandemic data is challenging since risk adjustment may not be reliable. Also, data from resources may not be available due to urgent actions of resource allocation. Therefore, future studies should explore reliable metrics of COVID-19 outcomes, and new methodologies to compare units with respect to the treatment of those patients.

- Risk-adjustment for COVID-19 patients: Following the challenges on benchmarking units with COVID-19 patents, risk-adjustment should be improved. For instance, traditional severity of illness scores such as the SAPS-3 and APACHE are not well-suitable for indicating mortality risks in COVID-19 patients.

- Following the benchmarking approach, understanding practices associated with good patient outcomes is also essential in identifying targets for improvement in the unit's organizational aspects. We suggest futures studies to address organizational factors and understanding what practices were associated with better outcomes for COVID-19 patients 
- The previously mentioned studies can also be extended to other diagnosis, in the ICU or hospital contexts. We encourage those models and methods to be applied for improving benchmarking and assisting the healthcare processes. Future studies can also evaluate the impact of COVID-19 admissions in other diagnosis in terms of outcomes and resources. 


\section{Publications}

Author's $h$-index by Scopus (September, 2021) $=6$

\section{Articles published in this thesis}

\begin{tabular}{|l|l|l|l|}
\hline Article & $\begin{array}{l}\text { Impact } \\
\text { factor }\end{array}$ & $\begin{array}{l}\text { ScoPUS's } \\
\text { percentile }\end{array}$ & $\begin{array}{l}\text { CAPES } \\
\text { Qualis }\end{array}$ \\
\hline $\begin{array}{l}\text { BASTOS, L.S.L. et al. Structure and process } \\
\text { associated with the efficiency of intensive care units } \\
\text { in low-resource settings: An analysis of the } \\
\text { CHECKLIST-ICU trial database. Journal of Critical } \\
\text { Care, v. 59, p. 118-123, out. 2020. }\end{array}$ & $\begin{array}{l}\mathbf{3 . 4 2 5} \\
\mathbf{2 0 2 0 )}\end{array}$ & $\mathbf{8 8}$ & \\
\hline $\begin{array}{l}\text { RANZANI, O.T., BASTOS, L.S.L et al. } \\
\text { Characterisation of the first 250 000 hospital } \\
\text { admissions for COVID-19 in Brazil: a retrospective } \\
\text { analysis of nationwide data. The Lancet } \\
\text { Respiratory Medicine, v. 9, n. 4, p. 407-418, abr. } \\
\text { 2021. }\end{array}$ & $\mathbf{3 0 2 0 )}$ & $\mathbf{A 1}$ & \\
\hline $\begin{array}{l}\text { BASTOS, L.S.L., RANZANI, O.T. et al. COVID-19 } \\
\text { hospital admissions: Brazil's first and second waves } \\
\text { compared. The Lancet Respiratory Medicine, v. 9, } \\
\text { n. 8, p. e82-e83, ago. 2021. }\end{array}$ & $\begin{array}{l}\mathbf{3 0 . 7 0 0} \\
\mathbf{( 2 0 2 0 )}\end{array}$ & $\mathbf{9 9}$ & $\mathbf{A 1}$ \\
\hline $\begin{array}{l}\text { KURTZ, P., BASTOS, L.S.L. et al. Evolving changes } \\
\text { in mortality of 13,301 critically ill adult patients with } \\
\text { COVID-19 over 8 months. Intensive care medicine, } \\
\text { v. 47, n. 5, p. 538-548, 2021a. }\end{array}$ & $\begin{array}{l}\mathbf{( 2 0 2 0 )} \\
\text { (20240 }\end{array}$ & $\mathbf{9 8}$ & $\mathbf{A 1}$ \\
\hline
\end{tabular}

\section{Other articles published}

\begin{tabular}{|l|l|l|l|}
\hline Article & $\begin{array}{l}\text { Impact } \\
\text { factor }\end{array}$ & $\begin{array}{l}\text { ScOPUS's } \\
\text { percentile }\end{array}$ & $\begin{array}{l}\text { CAPES } \\
\text { Qualis }\end{array}$ \\
\hline $\begin{array}{l}\text { ZAMPIERI, F.G.Z, BASTOS, L.S.L, et al. The } \\
\text { association of the COVID-19 pandemic and short- } \\
\text { term outcomes of non-COVID-19 critically ill } \\
\text { patients: an observational cohort study in Brazilian } \\
\text { ICUs. Intensive Care Medicine, 2021. }\end{array}$ & $\mathbf{9 8}$ & $\mathbf{A 1}$ \\
\hline $\begin{array}{l}\text { KURTZ, P., BASTOS, L.S.L, et al. SAPS-3 } \\
\text { performance for hospital mortality prediction in } \\
\text { 30,571 patients with COVID-19 admitted to ICUs in } \\
\text { Brazil. Intensive Care Medicine, 10 jul. 2021a. }\end{array}$ & \begin{tabular}{l} 
(2020) \\
\hline
\end{tabular} & $\mathbf{9 8}$ & $\mathbf{A 1}$ \\
\hline
\end{tabular}




\begin{tabular}{|c|c|c|c|}
\hline $\begin{array}{l}\text { PERES, I. T. et al. Sociodemographic factors } \\
\text { associated with COVID-19 in-hospital mortality in } \\
\text { Brazil. Public Health, v. 192, p. 15-20, mar. } 2021 .\end{array}$ & $\begin{array}{l}1.774 \\
(2019)\end{array}$ & 69 & A3 \\
\hline $\begin{array}{l}\text { DANTAS, L. F. et al. App-based symptom tracking } \\
\text { to optimize SARS-CoV-2 testing strategy using } \\
\text { machine learning. PLOS ONE, v. 16, n. 3, p. } \\
\text { e0248920, } 25 \text { mar. } 2021 .\end{array}$ & 2.74 (2019) & 92 & A1 \\
\hline $\begin{array}{l}\text { KURTZ, P. et al. Effect of Convalescent Plasma in } \\
\text { Critically III Patients With COVID-19: An } \\
\text { Observational Study. Frontiers in Medicine, v. 8, p. } \\
3,2021 \mathrm{~b} \text {. }\end{array}$ & 3.9 (2019) & 86 & A2 \\
\hline $\begin{array}{l}\text { ANTUNES, B. B. DE P. et al. Progression of } \\
\text { confirmed COVID-19 cases after the implementation } \\
\text { of control measures. Revista Brasileira de terapia } \\
\text { intensiva, v. 32, n. 2, p. } 213-223,2020 \text {. }\end{array}$ & $\begin{array}{l}1.397 \\
(2019-2020)\end{array}$ & 54 & A4 \\
\hline $\begin{array}{l}\text { PRADO, M. F. DO et al. Analysis of COVID-19 } \\
\text { under-reporting in Brazil. Revista Brasileira de } \\
\text { Terapia Intensiva, v. } 32, \text { n. } 2,2020 \text {. }\end{array}$ & $\begin{array}{l}1.397 \\
(2019-2020)\end{array}$ & 54 & A4 \\
\hline $\begin{array}{l}\text { KURTZ, P. et al. Effect of seasonal and temperature } \\
\text { variation on hospitalizations for stroke over a 10- } \\
\text { year period in Brazil. International Journal of } \\
\text { Stroke, p. } 1747493020947333,2020 \text { a. }\end{array}$ & $\begin{array}{l}4.882 \\
(2019-2020)\end{array}$ & 88 & A1 \\
\hline $\begin{array}{l}\text { KURTZ, P. et al. Systemic Severity and Organ } \\
\text { Dysfunction in Subarachnoid Hemorrhage: A Large } \\
\text { Retrospective Multicenter Cohort Study. } \\
\text { Neurocritical care, p. } 1-6,2020 \text { b. }\end{array}$ & $\begin{array}{l}2.720 \\
(2019)\end{array}$ & 81 & A2 \\
\hline $\begin{array}{l}\text { MELO, A. C. S. et al. Frameworks for reverse } \\
\text { logistics and sustainable design integration under a } \\
\text { sustainability perspective: a systematic literature } \\
\text { review. Research in Engineering Design, p. 1-19, } \\
2020 \text {. }\end{array}$ & $\begin{array}{l}2.224 \\
(2019)\end{array}$ & 86 & A2 \\
\hline $\begin{array}{l}\text { BASTOS, L. S. et al. A mixed integer programming } \\
\text { approach to the patient admission scheduling } \\
\text { problem. European Journal of Operational } \\
\text { Research, v. } 273, \text { n. 3, p. } 831-840,2019 \text {. }\end{array}$ & $\begin{array}{l}4.213 \\
(2019)\end{array}$ & 94 & A1 \\
\hline $\begin{array}{l}\text { ANTUNES, B. B. et al. A solution framework } \\
\text { based on process mining, optimization, and } \\
\text { discrete-event simulation to improve queue } \\
\text { performance in an emergency department. } \\
\text { International Conference on Business Process } \\
\text { Management. Anais...Springer, Cham, } 2019 \text {. }\end{array}$ & $\begin{array}{l}0.943 \\
(2019)\end{array}$ & 29 & B2 \\
\hline
\end{tabular}




\section{References}

ALI, M. S. et al. Administrative Data Linkage in Brazil: Potentials for Health Technology Assessment. Frontiers in Pharmacology, v. 10, p. 984, 23 set. 2019.

ALTMAN, D. G.; ROYSTON, P. The cost of dichotomising continuous variables. BMJ, v. 332, n. 7549, p. 1080.1, 6 maio 2006.

AMARAL, P. V. et al. Spatially balanced provision of health equipment: a crosssectional study oriented to the identification of challenges to access promotion. International Journal for Equity in Health, v. 16, n. 1, p. 209, dez. 2017.

AMBROSIUS, W. T. (ED.). Topics in biostatistics. Totowa, N.J: Humana Press, 2007.

ANGUS, D. C. et al. Effect of Hydrocortisone on Mortality and Organ Support in Patients With Severe COVID-19: The REMAP-CAP COVID-19 Corticosteroid Domain Randomized Clinical Trial. JAMA, 2 set. 2020.

ARABI, Y. M.; FOWLER, R.; HAYDEN, F. G. Critical care management of adults with community-acquired severe respiratory viral infection. Intensive Care Medicine, v. 46, n. 2, p. 315-328, fev. 2020.

ATHEY, S.; IMBENS, G. Recursive partitioning for heterogeneous causal effects. Proceedings of the National Academy of Sciences of the United States of America, v. 113, n. 27, p. 7353-7360, 2016.

ATHEY, S.; TIBSHIRANI, J.; WAGER, S. Generalized random forests. The Annals of Statistics, v. 47, n. 2, 1 abr. 2019.

AUSTIN, S. et al. Access to urban acute care services in high- vs. middleincome countries: an analysis of seven cities. Intensive Care Medicine, v. 40, n. 3, p. 342-352, mar. 2014.

AYANIAN, J. Z.; MARKEL, H. Donabedian's Lasting Framework for Health Care Quality. New England Journal of Medicine, v. 375, n. 3, p. 205-207, 21 jul. 2016.

AZEVEDO, L. C. et al. Clinical outcomes of patients requiring ventilatory support in Brazilian intensive care units: a multicenter, prospective, cohort study. Critical Care, v. 17, n. 2, p. R63, abr. 2013. 
BAQUI, P. et al. Ethnic and regional variations in hospital mortality from COVID19 in Brazil: a cross-sectional observational study. The Lancet Global Health, v. 8, n. 8, p. e1018-e1026, ago. 2020.

BARRETT, T. S.; LOCKHART, G. Efficient Exploration of Many Variables and Interactions Using Regularized Regression. Prevention Science, v. 20, n. 4, p. 575-584, maio 2019.

BASTOS, L. S. et al. COVID-19 and hospitalizations for SARI in Brazil: a comparison up to the 12th epidemiological week of 2020. Cadernos de Saúde Pública, v. 36, n. 4, p. e00070120, 2020a.

BASTOS, L. S. L. et al. Structure and process associated with the efficiency of intensive care units in low-resource settings: An analysis of the CHECKLISTICU trial database. Journal of Critical Care, v. 59, p. 118-123, out. 2020b.

BLAND, J. M. Statistics Notes: The odds ratio. BMJ, v. 320, n. 7247, p. 14681468, 27 maio 2000.

BOEHMER, T. K. et al. Changing Age Distribution of the COVID-19 Pandemic - United States, May-August 2020. MMWR. Morbidity and Mortality Weekly Report, v. 69, n. 39, p. 1404-1409, 2 out. 2020.

BOZZA, F. A.; SALLUH, J. I. An urban perspective on sepsis in developing countries. The Lancet Infectious Diseases, v. 10, n. 5, p. 290-291, maio 2010.

BUUREN, S. VAN; GROOTHUIS-OUDSHOORN, K. mice: Multivariate Imputation by Chained Equations in R. Journal of Statistical Software, v. 45, n. 3, 2011.

CANDIDO, D. S. et al. Evolution and epidemic spread of SARS-CoV-2 in Brazil. Science, v. 369, n. 6508, p. 1255-1260, 4 set. 2020.

CAO, B. et al. A Trial of Lopinavir-Ritonavir in Adults Hospitalized with Severe Covid-19. New England Journal of Medicine, v. 382, n. 19, p. 1787-1799, 7 maio 2020.

CARPENTER, J.; BITHELL, J. Bootstrap confidence intervals: When, which, what? A practical guide for medical statisticians. Statistics in Medicine, 2000.

CAVALCANTI, A. B. et al. Effect of a Quality Improvement Intervention With Daily Round Checklists, Goal Setting, and Clinician Prompting on Mortality of Critically III Patients: A Randomized Clinical Trial. JAMA, v. 315, n. 14, p. 1480 1490, 2016.

CENTRE FOR GLOBAL INFECTIOUS DISEASE ANALYSIS, IMPERIAL COLLEGE LONDON. Future scenarios of the healthcare burden of COVID19 in Low- or Middle-Income Countries. Disponível em: <https://mrcide.github.io/global-Imic-reports/>. 
CHALLEN, R. et al. Risk of mortality in patients infected with SARS-CoV-2 variant of concern 202012/1: matched cohort study. BMJ, p. n579, 9 mar. 2021.

CHATTERJEE, A.; LAHIRI, S. N. Bootstrapping lasso estimators. Journal of the American Statistical Association, 2011.

CHECKLEY, W. et al. Structure, process, and annual ICU mortality across 69 centers: United States critical illness and injury trials group critical illness outcomes study. Critical Care Medicine, v. 42, n. 2, p. 344-356, 2014.

CHIPMAN, H. A.; GEORGE, E. I.; MCCULLOCH, R. E. BART: Bayesian additive regression trees. Annals of Applied Statistics, 2012.

CNES - CADASTRO NACIONAL DE ESTABELECIMENTOS DE SAÚDE. CNES Official website. Disponível em:

$<$ http://cnes.datasus.gov.br/pages/downloads/arquivosBaseDados.jsp>. Acesso em: 1 set. 2020.

COX, D. R. Regression Models and Life-Tables. Journal of the Royal Statistical Society: Series B (Methodological), v. 34, n. 2, p. 187-202, jan. 1972.

DAMIANI, L. P. et al. A cluster-randomised trial of a multifaceted quality improvement intervention in Brazilian intensive care units (Checklist-ICU trial): Statistical analysis plan. Critical Care and Resuscitation, v. 17, n. 2, p. 113121, 2015.

DANTAS, L. F. et al. App-based symptom tracking to optimize SARS-CoV-2 testing strategy using machine learning. PLOS ONE, v. 16, n. 3, p. e0248920, 25 mar. 2021.

DATASUS. Severe Acute Respiratory Infection data in Brasil: Influenza Epidemiological Surveillance Information System (SIVEP-Gripe).

Disponível em: <https://opendatasus.saude.gov.br/dataset/bd-srag-2020>. Acesso em: 10 ago. 2020.

DAWSON, N. V.; WEISS, R. Dichotomizing Continuous Variables in Statistical Analysis: A Practice to Avoid. Medical Decision Making, v. 32, n. 2, p. 225226, mar. 2012.

DE LANGE, D. W.; DONGELMANS, D. A.; DE KEIZER, N. F. Small steps beyond benchmarking. Revista Brasileira de Terapia Intensiva, v. 29, n. 2, p. 128-130, 2017.

DOCHERTY, A. B. et al. Features of 20133 UK patients in hospital with covid19 using the ISARIC WHO Clinical Characterisation Protocol: prospective observational cohort study. BMJ, p. m1985, 22 maio 2020.

DONABEDIAN, A. The quality of care. How can it be assessed? JAMA: The Journal of the American Medical Association, v. 260, n. 12, p. 1743-1748, 23 set. 1988. 
DONDORP, A. M. et al. Respiratory Support in COVID-19 Patients, with a Focus on Resource-Limited Settings. The American Journal of Tropical Medicine and Hygiene, v. 102, n. 6, p. 1191-1197, 3 jun. 2020.

DONG, E.; DU, H.; GARDNER, L. An interactive web-based dashboard to track COVID-19 in real time. The Lancet Infectious Diseases, v. 20, n. 5, p. 533534, maio 2020.

DRESCH, A.; LACERDA, D. P.; ANTUNES JR, J. A. V. Design Science Research: A Method for Science and Technology Advancement. Cham: Springer International Publishing, 2015.

ESPINOZA, R. et al. Factors associated with mortality in severe communityacquired pneumonia: A multicenter cohort study. Journal of Critical Care, v. 50, p. 82-86, abr. 2019.

FAN, E. et al. COVID-19-associated acute respiratory distress syndrome: is a different approach to management warranted? The Lancet Respiratory Medicine, v. 8, n. 8, p. 816-821, ago. 2020.

FARIA, N. R. et al. Genomics and epidemiology of the P.1 SARS-CoV-2 lineage in Manaus, Brazil. Science, v. 372, n. 6544, p. 815-821, 21 maio 2021.

FARRINGTON, D. P.; LOEBER, R. Some benefits of dichotomization in psychiatric and criminological research. Criminal Behaviour and Mental Health, v. 10, n. 2, p. 100-122, jun. 2000.

FINAZZI, S. et al. Calibration Belt for Quality-of-Care Assessment Based on Dichotomous Outcomes. PLoS ONE, v. 6, n. 2, p. e16110, 23 fev. 2011.

FLAATTEN, $H$. The present use of quality indicators in the intensive care unit: Quality indicators in the ICU. Acta Anaesthesiologica Scandinavica, v. 56, n. 9, p. 1078-1083, out. 2012.

FREITAS, A. R. R. et al. Tracking excess deaths associated with the COVID-19 epidemic as an epidemiological surveillance strategy-preliminary results of the evaluation of six Brazilian capitals. Revista da Sociedade Brasileira de Medicina Tropical, v. 53, p. e20200558, 2020.

FRIEDMAN, J.; HASTIE, T.; TIBSHIRANI, R. Regularization Paths for Generalized Linear Models via Coordinate Descent. Journal of statistical software, v. 33, n. 1, p. 1-22, 2010.

GARLAND, A. Improving the ICU: Part 2. Chest, v. 127, n. 6, p. 2165-2179, jun. 2005.

GOOGLE LLC. Google Mobility Reports. Disponível em: <https://www.google.com/covid19/mobility/>. Acesso em: 22 maio. 2021.

GRASSELLI, G. et al. Risk Factors Associated With Mortality Among Patients With COVID-19 in Intensive Care Units in Lombardy, Italy. JAMA Internal Medicine, 15 jul. 2020. 
GREIFER, N. Weightlt: Weighting for Covariate Balance in Observational Studies. Disponível em: <https://cran.rproject.org/web/packages/Weightlt/index.html>. Acesso em: 10 jun. 2020.

GUO, S.; FRASER, M. W. Propensity score analysis: statistical methods and applications. Thousand Oaks, Calif: Sage Publications, 2010.

GUPTA, S. et al. Factors Associated With Death in Critically III Patients With Coronavirus Disease 2019 in the US. JAMA Internal Medicine, 15 jul. 2020.

HADLEY WICKHAM. ggplot2: Elegant Graphics for Data Analysis.

Disponível em: <https://ggplot2-book.org/>.

HALLAL, P. C. et al. SARS-CoV-2 antibody prevalence in Brazil: results from two successive nationwide serological household surveys. The Lancet Global Health, v. 8, n. 11, p. e1390-e1398, nov. 2020.

HARRELL, J. Regression Modeling Strategies: With Applications to Linear Models, Logistic and Ordinal Regression, and Survival Analysis. 2nd ed. 2015 ed. Cham: Springer International Publishing : Imprint: Springer, 2015.

HASTIE, T.; TIBSHIRANI, R.; FRIEDMAN, J. The elements of statistical learning: data mining, inference, and prediction. [s.l.] Springer Science \& Business Media, 2009.

HEINZE, G.; WALLISCH, C.; DUNKLER, D. Variable selection - A review and recommendations for the practicing statistician. Biometrical Journal, v. 60, n. 3, p. 431-449, 2018.

HEVNER, A. R. A Three Cycle View of Design Science Research.

Scandinavian Journal of Information Systems, v. 2, n. 19, p. 87-92, 2007.

HEWSON-CONROY, K. M.; ELLIOTT, D.; BURRELL, A. R. Quality and safety in intensive care-A means to an end is critical. Australian Critical Care, v. 23, n. 3, p. 109-129, 1 ago. 2010.

HIRANO, K.; IMBENS, G. W. The propensity score with continuous treatments. In: GELMAN, A.; MENG, X.-L. (Eds.). . Applied Bayesian modeling and causal inference from incomplete-data perspectives : an essential journey with Donald Rubin's statistical family. [s.I.] John Wiley, 2004. p. 73-84.

HIRZALLAH, F. M.; ALKAISSI, A.; DO CÉU BARBIERI-FIGUEIREDO, M. A systematic review of nurse-led weaning protocol for mechanically ventilated adult patients. Nursing in Critical Care, v. 24, n. 2, p. 89-96, 2019.

JALILI, M. et al. Characteristics and Mortality of Hospitalized Patients With COVID-19 in Iran: A National Retrospective Cohort Study. Annals of Internal Medicine, p. M20-2911, 20 jul. 2020.

JAMES, G. et al. Introduction to Statistical Learning: with applications in $\mathbf{r}$. S.I.: SPRINGER-VERLAG NEW YORK, 2021. 
KARAGIANNIDIS, C. et al. Case characteristics, resource use, and outcomes of 10021 patients with COVID-19 admitted to 920 German hospitals: an observational study. The Lancet Respiratory Medicine, $p$. S2213260020303167, jul. 2020.

KEEGAN, M. T.; GAJIC, O.; AFESSA, B. Severity of illness scoring systems in the intensive care unit: Critical Care Medicine, v. 39, n. 1, p. 163-169, jan. 2011.

KLEINBAUM, D. G.; KLEIN, M. Survival analysis: a self-learning text. 3rd ed ed. New York: Springer, 2012.

KLOMPAS, M. What is new in the prevention of nosocomial pneumonia in the ICU? Current Opinion in Critical Care, v. 23, n. 5, p. 378-384, 2017.

KOTRLIK, J.; WILLIAMS, H. The Incorporation of Effect Size in Information Technology, Learning, and Performance Research. Information Technology, Learning, and Performance Journal, v. 21, n. 1, p. 1-7, 2003.

KURTH, T. et al. Results of Multivariable Logistic Regression, Propensity Matching, Propensity Adjustment, and Propensity-based Weighting under Conditions of Nonuniform Effect. American Journal of Epidemiology, v. 163, n. 3, p. 262-270, 1 fev. 2006.

LANGE, D. W. DE; DONGELMANS, D. A.; KEIZER, N. F. DE. Small steps beyond benchmarking. Revista Brasileira de Terapia Intensiva, v. 29, n. 2, 2017.

LARSON, G. E.; MCKEEVER, S. Nurse titrated analgesia and sedation in intensive care increases the frequency of comfort assessment and reduces midazolam use in paediatric patients following cardiac surgery. Australian Critical Care, v. 31, n. 1, p. 31-36, 2018.

LATIF, A. A. et al. S:E484K Mutation Report. [s.I: s.n.]. Disponível em: <https://outbreak.info/situation-reports?muts=S\%3AE484K>. Acesso em: 31 mar. 2021.

LEMOS, D. R. Q. et al. Health system collapse 45 days after the detection of COVID-19 in Ceará, Northeast Brazil: a preliminary analysis. Revista da Sociedade Brasileira de Medicina Tropical, v. 53, p. e20200354, 2020.

LOCKHART, R. et al. A significance test for the lassoAnnals of Statistics, 2014.

MACCALLUM, R. C. et al. On the practice of dichotomization of quantitative variables. Psychological Methods, v. 7, n. 1, p. 19-40, 2002.

MACHADO, F. R. et al. The epidemiology of sepsis in Brazilian intensive care units (the Sepsis PREvalence Assessment Database, SPREAD): an observational study. The Lancet Infectious Diseases, v. 17, n. 11, p. 11801189, nov. 2017. 
MADLEY-DOWD, $P$. et al. The proportion of missing data should not be used to guide decisions on multiple imputation. Journal of Clinical Epidemiology, $v$. 110, p. 63-73, jun. 2019.

MALDONADO, G.; GREENLAND, S. Simulation study of confounder-selection strategies. American Journal of Epidemiology, 1993.

MANRESA PEREZ, A. Machine Learning to Predict High-cost

Hospitalizations. MESTRE EM ENGENHARIA DE PRODUÇÃO-Rio de Janeiro, Brazil: PONTIFÍCIA UNIVERSIDADE CATÓLICA DO RIO DE JANEIRO, 20 mar. 2020.

MARINHO, F. et al. Burden of disease in Brazil, 1990-2016: a systematic subnational analysis for the Global Burden of Disease Study 2016. The Lancet, v. 392, n. 10149, p. 760-775, set. 2018.

MASSUDA, A. et al. The Brazilian health system at crossroads: progress, crisis and resilience. BMJ Global Health, v. 3, n. 4, p. e000829, jul. 2018.

MCCLEAN, K. et al. Identification and assessment of potentially high-mortality intensive care units using the ANZICS Centre for Outcome and Resource Evaluation clinical registry. Critical care and resuscitation : journal of the Australasian Academy of Critical Care Medicine, v. 19, n. 3, p. 230-238, 2017.

MERZ, T. M. et al. Resource use and outcome in critically ill patients with hematological malignancy: a retrospective cohort study. Critical care (London, England), 2008.

METNITZ, P. G. H. et al. SAPS 3-From evaluation of the patient to evaluation of the intensive care unit. Part 1: Objectives, methods and cohort description. Intensive Care Medicine, v. 31, n. 10, p. 1336-1344, out. 2005.

MICKEY, R. M.; GREENLAND, S. The impact of confounder selection criteria on effect estimation. American Journal of Epidemiology, 1989.

MIDEGA, T. D. et al. Organizational factors associated with adherence to low tidal volume ventilation: a secondary analysis of the CHECKLIST-ICU database. Annals of Intensive Care, v. 10, n. 1, p. 68, dez. 2020.

MORALEZ, G. M. et al. External validation of SAPS 3 and MPM0-III scores in 48,816 patients from 72 Brazilian ICUs. Annals of Intensive Care, v. 7, n. 1, p. 53, 2017.

MORENO, R. P. et al. SAPS 3-From evaluation of the patient to evaluation of the intensive care unit. Part 2: Development of a prognostic model for hospital mortality at ICU admission. Intensive Care Medicine, v. 31, n. 10, p. 13451355, out. 2005.

MURTHY, S.; LELIGDOWICZ, A.; ADHIKARI, N. K. J. Intensive Care Unit Capacity in Low-Income Countries: A Systematic Review. PLOS ONE, v. 10, n. 1, p. e0116949, 24 jan. 2015. 
ÑAMENDYS-SILVA, S. A.; GUTIÉRREZ-VILLASEÑOR, A.; ROMEROGONZÁLEZ, J. P. Hospital mortality in mechanically ventilated COVID-19 patients in Mexico. Intensive Care Medicine, v. 46, n. 11, p. 2086-2088, nov. 2020.

NASSAR, A. P. et al. Organizational factors associated with target sedation on the first $48 \mathrm{~h}$ of mechanical ventilation: an analysis of checklist-ICU database. Critical Care, v. 23, n. 1, p. 34, dez. 2019.

NATHANSON, B. H. et al. A revised method to assess intensive care unit clinical performance and resource utilization*: Critical Care Medicine, v. 35, n. 8, p. 1853-1862, ago. 2007.

NATTINO, G. et al. Assessing the Calibration of Dichotomous Outcome Models with the Calibration Belt. The Stata Journal: Promoting communications on statistics and Stata, v. 17, n. 4, p. 1003-1014, 2017 a.

NATTINO, G. et al. The GiViTI Calibration Test and Belt. Disponível em: $<$ https://cran.r-project.org/web/packages/givitiR/givitiR.pdf>. Acesso em: 5 ago. 2019b.

NEWGARD, C. D. et al. Validation of length of hospital stay as a surrogate measure for injury severity and resource use among injury survivors. Academic Emergency Medicine, 2010.

NISBET, R.; ELDER, J.; MINER, G. Handbook of Statistical Analysis and Data Mining Applications. [s.I.] Elsevier, 2009.

NISKANEN, M.; REINIKAINEN, M.; PETTILÄ, V. Case-mix-adjusted length of stay and mortality in 23 Finnish ICUs. Intensive Care Medicine, v. 35, n. 6, p. 1060-1067, 2009.

NOUIRA, $\mathrm{H}$. et al. Which indicators used to assess quality performance in Intensive Care Units? A systematic review of medical literature. Anaesthesia Critical Care and Pain Medicine, 2018.

NUZZO, R. L. Making Continuous Measurements into Dichotomous Variables. PM\&R, v. 11, n. 10, p. 1132-1134, out. 2019.

ORELLANA, J. D. Y. et al. Explosion in mortality in the Amazonian epicenter of the COVID-19 epidemic. Cadernos de Saúde Pública, v. 36, n. 7, p. e00120020, 2020.

ORGANIZATION, W. H. Clinical management of severe acute respiratory infection (SARI) when COVID-19 disease is suspected: interim guidance, 13 March 2020. [s.l.] World Health Organization, 2020.

PERES, I. T. et al. Sociodemographic factors associated with COVID-19 inhospital mortality in Brazil. Public Health, v. 192, p. 15-20, mar. 2021.

PERKINS, G. D. et al. RECOVERY- Respiratory Support: Respiratory

Strategies for patients with suspected or proven COVID-19 respiratory failure; 
Continuous Positive Airway Pressure, High-flow Nasal Oxygen, and standard care: A structured summary of a study protocol for a randomised controlled trial. Trials, v. 21, n. 1, p. 687, dez. 2020.

POOLE, D. et al. Comparison between SAPS II and SAPS 3 in predicting hospital mortality in a cohort of 103 Italian ICUs. Is new always better? Intensive Care Medicine, v. 38, n. 8, p. 1280-1288, 2012.

R CORE TEAM. R: A Language and Environment for Statistical

Computing. Vienna, Austria: R Foundation for Statistical Computing, 2021.

RANZANI, O. T. et al. Characterisation of the first 250000 hospital admissions for COVID-19 in Brazil: a retrospective analysis of nationwide data. The Lancet Respiratory Medicine, v. 9, n. 4, p. 407-418, abr. 2021.

RAPOPORT, J. et al. A method for assessing the clinical performance and costeffectiveness of intensive care units: a multicenter inception cohort study.

Critical care medicine, v. 22, n. 9, p. 1385-1391, 1994.

REEVES, D. et al. Combining Multiple Indicators of Clinical Quality: An Evaluation of Different Analytic Approaches. Medical Care, v. 45, n. 6, p. 489496, jun. 2007.

ROSENBAUM, P. R.; RUBIN, D. B. The central role of the propensity score in observational studies for causal effects. Biometrika, v. 70, n. 1, p. 41-55, 1983.

ROTHEN, H. U. et al. Variability in outcome and resource use in intensive care units. Intensive Care Medicine, v. 33, n. 8, p. 1329-1336, 23 jul. 2007.

ROTHEN, H. U.; TAKALA, J. Can outcome prediction data change patient outcomes and organizational outcomes?: Current Opinion in Critical Care, v. 14, n. 5, p. 513-519, out. 2008.

RUBIN, D. B. Multiple imputation for nonresponse in surveys. New York: Wiley, 1987.

SALLUH, J. I. F. et al. New perspectives to improve critical care benchmarking. Annals of Intensive Care, v. 8, n. 1, p. 17, dez. 2018.

SALLUH, J. I. F.; LISBOA, T. Critical Care in Brazil. v. 16, n. 3, 2016.

SALLUH, J. I. F.; LISBOA, T.; BOZZA, F. A. Challenges for the care delivery for critically ill COVID-19 patients in developing countries: the Brazilian perspective. Critical Care, v. 24, n. 1, p. 593, dez. 2020.

SALLUH, J. I. F.; SOARES, M.; KEEGAN, M. T. Understanding intensive care unit benchmarking. Intensive Care Medicine, v. 43, n. 11, p. 1703-1707, nov. 2017.

SALLUH, J. I. F.; SOARES, M.; SINGER, M. Spreading the knowledge on the epidemiology of sepsis. The Lancet Infectious Diseases, v. 17, n. 11, p. 1104-1106, 2017. 
SALYER, S. J. et al. The first and second waves of the COVID-19 pandemic in Africa: a cross-sectional study. The Lancet, p. S0140673621006322, mar. 2021.

SCHMIDT, M. et al. Clinical characteristics and day-90 outcomes of 4244 critically ill adults with COVID-19: a prospective cohort study. Intensive Care Medicine, v. 47, n. 1, p. 60-73, 1 jan. 2021.

SHEARER, C. The CRISP-DM model: the new blueprint for data mining. Journal of data warehousing, v. 5, n. 4, p. 13-22, 2000.

SILVA JUNIOR, J. M. et al. Applicability of the simplified acute physiology score (SAPS 3) in Brazilian hospitals. Revista brasileira de anestesiologia, v. 60, n. 1, p. 20-31, 2010.

SOARES, M. et al. Organizational characteristics, outcomes, and resource use in 78 Brazilian intensive care units: the ORCHESTRA study. Intensive Care Medicine, v. 41, n. 12, p. 2149-2160, dez. 2015.

SOARES, M. et al. Family care, visiting policies, ICU performance, and efficiency in resource use: insights from the ORCHESTRA study. Intensive Care Medicine, v. 43, n. 4, p. 590-591, 2017.

SONENTHAL, P. D. et al. COVID-19 preparedness in Malawi: a national facilitybased critical care assessment. The Lancet Global Health, v. 8, n. 7, p. e890e892, jul. 2020.

SOPEYIN, A. et al. Transmission risk of respiratory viruses in natural and mechanical ventilation environments: implications for SARS-CoV-2 transmission in Africa. BMJ Global Health, v. 5, n. 8, p. e003522, ago. 2020.

SPIEGELHALTER, D. J. Funnel plots for comparing institutional performance. Statistics in Medicine, v. 24, n. 8, p. 1185-1202, 30 abr. 2005.

STERNE, J. A. C. et al. Association Between Administration of Systemic Corticosteroids and Mortality Among Critically III Patients With COVID-19: A Meta-analysis. JAMA, v. 324, n. 13, p. 1330, 6 out. 2020.

SZWARCWALD, C. L. et al. Inequalities in healthy life expectancy by Brazilian geographic regions: findings from the National Health Survey, 2013.

International Journal for Equity in Health, v. 15, n. 1, p. 141, dez. 2016.

TAYLOR, L. How Latin America is fighting covid-19, for better and worse. BMJ, p. $\mathrm{m} 3319,1$ set. 2020.

THERNEAU, T. M. et al. survival: Survival Analysis. Disponível em: $<$ https://ourworldindata.org/grapher/total-healthcare-expenditure-as-share-ofnational-gdp-by-country>. Acesso em: 13 set. 2021.

THERNEAU, TERRY M. coxme: Mixed Effects Cox Models. Disponível em: $<$ https://cran.r-project.org/web/packages/coxme/index.html>. 
TOMAZINI, B. M. et al. Effect of Dexamethasone on Days Alive and VentilatorFree in Patients With Moderate or Severe Acute Respiratory Distress Syndrome and COVID-19: The CoDEX Randomized Clinical Trial. JAMA, 2 set. 2020.

TZOTZOS, S. J. et al. Incidence of ARDS and outcomes in hospitalized patients with COVID-19: a global literature survey. Critical Care, v. 24, n. 1, p. 516, dez. 2020.

VAN DAMME, W. et al. The COVID-19 pandemic: diverse contexts; different epidemics-how and why? BMJ Global Health, v. 5, n. 7, p. e003098, jul. 2020.

VAN DE KLUNDERT, N. et al. Data Resource Profile: the Dutch National Intensive Care Evaluation (NICE) Registry of Admissions to Adult Intensive Care Units. International Journal of Epidemiology, v. 44, n. 6, p. 18501850h, dez. 2015.

VERBURG, I. W. M. et al. The association between outcome-based quality indicators for intensive care units. PLOS ONE, v. 13, n. 6, p. e0198522, 13 jun. 2018.

VUKOJA, M. et al. A Survey on Critical Care Resources and Practices in Lowand Middle-Income Countries. Global Heart, v. 9, n. 3, p. 337- 342.e5, 1 set. 2014.

WAGER, S.; ATHEY, S. Estimation and Inference of Heterogeneous Treatment Effects using Random Forests. Journal of the American Statistical Association, v. 113, n. 523, p. 1228-1242, 2018.

WALKER, S. H.; DUNCAN, D. B. Estimation of the Probability of an Event as a Function of Several Independent Variables. Biometrika, v. 54, n. 1/2, p. 167, jun. 1967.

WANG, Y. et al. Remdesivir in adults with severe COVID-19: a randomised, double-blind, placebo-controlled, multicentre trial. The Lancet, v. 395, n. 10236, p. 1569-1578, maio 2020.

WICKHAM, H. et al. Welcome to the Tidyverse. Journal of Open Source Software, v. 4, n. 43, p. 1686, 21 nov. 2019.

WICKHAM, H. et al. dplyr: A Grammar of Data Manipulation. Disponível em: $<$ https://cran.r-project.org/web/packages/dplyr/index.html>.

WINCK, J. C.; AMBROSINO, N. COVID-19 pandemic and non invasive respiratory management: Every Goliath needs a David. An evidence based evaluation of problems. Pulmonology, v. 26, n. 4, p. 213-220, jul. 2020.

WISE, J. Covid-19: The E484K mutation and the risks it poses. BMJ, p. n359, 5 fev. 2021.

WOODHOUSE, D. et al. Will benchmarking ICUs improve outcome?: Current Opinion in Critical Care, v. 15, n. 5, p. 450-455, out. 2009. 
WORDOMETER. Coronavirus. Disponível em:

<https://www.worldometers.info/coronavirus/>. Acesso em: 6 jun. 2021.

WORLD HEALTH ORGANIZATION. WHO Director-General's opening remarks at the media briefing on COVID-19 - 11 March 2020. Disponível em: $<$ https://www.who.int/director-general/speeches/detail/who-director-general-sopening-remarks-at-the-media-briefing-on-covid-19---11-march-2020>. Acesso em: 18 jun. 2021.

WORTEL, S. A. et al. Number of intensivists per bed is associated with efficiency of Dutch Intensive Care Units. 2020.

WORTEL, S. A. et al. Number of intensivists per bed is associated with efficiency of Dutch intensive care units. Journal of Critical Care, v. 62, p. 223229, abr. 2021.

XAVIER, D. R. et al. Health Regions in Brazil based on hospital admissions: a method to support health regionalization. Cadernos de Saúde Pública, v. 35, n. suppl 2, p. e00076118, 2019.

XIAO, N.; XU, Q. S. Multi-step adaptive elastic-net: reducing false positives in high-dimensional variable selection. Journal of Statistical Computation and Simulation, v. 85, n. 18, p. 3755-3765, 2015.

ZAMPIERI, F. G. et al. The Epimed Monitor ICU Database®: a cloud-based national registry for adult intensive care unit patients in Brazil. Revista Brasileira de Terapia Intensiva, v. 29, n. 4, 2017.

ZAMPIERI, F. G. et al. ICU staffing feature phenotypes and their relationship with patients' outcomes: an unsupervised machine learning analysis. Intensive Care Medicine, v. 45, n. 11, p. 1599-1607, nov. 2019.

ZEILEIS, A. et al. strucchange: An R Package for Testing for Structural Change in Linear Regression Models. Journal of Statistical Software, v. 7, n. 2, 2002.

ZHU, N. et al. A Novel Coronavirus from Patients with Pneumonia in China, 2019. New England Journal of Medicine, v. 382, n. 8, p. 727-733, 20 fev. 2020.

ZIMMERMAN, J. E. et al. Acute Physiology and Chronic Health Evaluation (APACHE) IV: Hospital mortality assessment for today's critically ill patients*: Critical Care Medicine, v. 34, n. 5, p. 1297-1310, maio 2006.

ZIMMERMAN, J. E.; ALZOLA, C.; VON RUEDEN, K. T. The use of benchmarking to identify top performing critical care units: A preliminary assessment of their policies and practices. Journal of Critical Care, v. 18, n. 2, p. 76-86, 2003.

ZOU, H.; HASTIE, T. Regularization and variable selection via the elastic net. Journal of the Royal Statistical Society. Series B: Statistical Methodology, v. 67, n. 2, p. 301-320, 2005. 
ZOU, H.; ZHANG, H. H. On the adaptive elastic-net with a diverging number of parameters. Annals of Statistics, v. 37, n. 4, p. 1733-1751, 2009. 


\section{Appendix}

12.1

Appendix A1

\subsection{1}

\section{Appendix A1.1 - Calibration of SAPS-3 curves and the Standardized Mortality Ratio (SMR)}

We measured mortality using the standardized mortality ratio (SMR), which assessed the observed-to-expected ratio of the mortality outcome for each ICU. The expected mortality was estimated using the standard SAPS-3 prognostic model equation (METNITZ et al., 2005).

To evaluate the calibration of the SAPS-3 risk curve to the CHECKLIST-ICU sample, we used the calibration belt method (NATTINO et al., 2017a). This method consists of fitting a function to relate the expected and observed mortality using an estimated curve and confidence intervals instead of discrete deciles comparison. Hence, the calibration plots are provided with confidence bands ("belts"), and the uncertainty around the model's estimation can be used to define possible under- or overestimation that may impact the mortality indicator or if the model follows the bisector line (estimated $=$ observed).

In our sample, the SAPS-3 standard equation did not provide a good calibration (Figure A11.1-a). It provided underestimates of mortality for low-risk patients (low observed mortality) and overestimates for high-risk patients, being the median SMR 1.23 (IQR, 0.85 to 1.65 ) using those estimates.

To diminish this effect, we performed first-level customization of the standard equation and observed an improvement in the model's calibration (Figure A11.1-b). The first-level customization consists in reestimating the coefficients from the reference equation using its probability values as a predictor in a new logistic regression with the outcomes from the study sample as the response. The confidence bands presented a small deviation to the bisector only for extreme values of severities. With the customization, the SMR distribution had median 1.01 (IQR, 0.71 to 1.25 ). 
The model and calibration plots were fitted to the data using the givitiR (NATTINO et al., 2017b) package for R.

Figure A12.1 - Calibration belts for evaluating the calibration of SAPS-3 standard equation

(a) SAPS3-SE

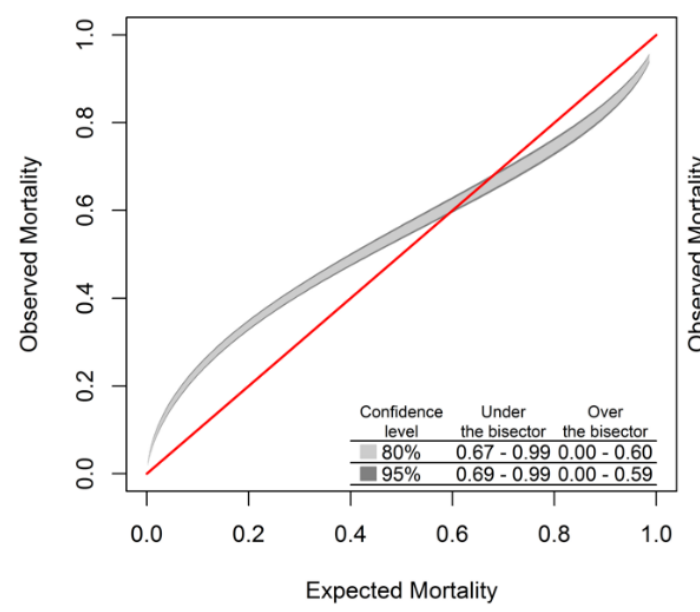

(b) SAPS3-SE (Custom)

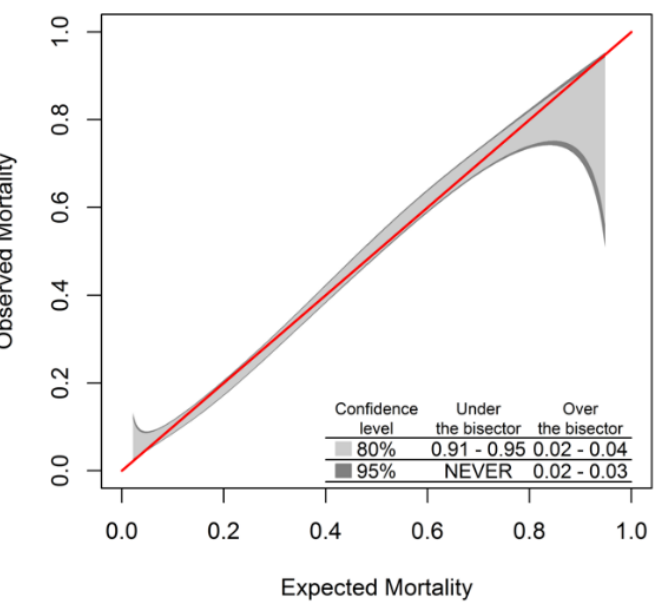

\subsection{2}

\section{Appendix A1.2 - Standardized Resouce Use (SRU) calculation}

We measured the resource utilization using the Standardized Resource Use (SRU), an observed-to-expected ratio. We followed the proposal of Rothen et al. (ROTHEN et al., 2007) and considered the ICU length-of-stay (ICU-LOS) as a surrogate measure of resource use. The authors used the SAPS-3 sample and obtained an average ICU-LOS per surviving patients for each decile of SAPS-3 as an "risk-adjusted use of resources", which is a common reference for calculating SRU.

However, in our study the CHECKLIST-ICU sample did not show a good "calibration" of the expected values (Table A11.1).

Table A12.1 - Comparison of average ICU length-of-stay per survivor patient and SAPS-3 deciles to calculate SRU

\begin{tabular}{lllll}
\hline & Rothen et al. 2007 & CHECKLIST-ICU & \\
SAPS-3 Decile & Surviving patients & ICU-LOS per survivor & Surviving patients & ICU-LOS per survivor \\
\hline$[16-24]$ & 517 & 2.3 & 467 & 5.9 \\
$(24-34]$ & 2494 & 3.2 & 1476 & 7.8 \\
$(34-44]$ & 3930 & 4.3 & 2241 & 9.9 \\
$(44-54]$ & 3284 & 7.2 & 2220 & 14.1 \\
$(54-64]$ & 2125 & 11 & 1436 & 20.9 \\
$(64-74]$ & 968 & 16.6 & 762 & 29.0 \\
$(74-84]$ & 358 & 22.2 & 328 & 42.4 \\
$(84-94]$ & 102 & 29.4 & 132 & 45.0 \\
$(94-137]$ & 31 & 39 & 50 & 63.5 \\
\hline
\end{tabular}

ICU-LOS - Length-of-Stay in the Intenstive Care Unit 
We observed that, in overall, the values from Rothen et al. underestimate the average LOS, then, comparatively, the CHECKLIST-ICU patients stayed longer in ICUs. Using those expected values, the SRU distribution has a median of 1.86 (IQR, 1.46 to 2.70).

To mitigate those effects, we considered the expected average ICU-LOS per survivor from the CHECKLIST-ICU sample itself. We used the sample SAPS-3 stratification from Rothen et al. to keep the same conditions from this study. SRU distribution had median 0.93 (IQR, 0.74 to 1.34 ). Although, it may not provide external validation, the those expected values can provide a fair "calibration" to the sample. We remind that there has not been a reference equation for prediction LOS given a severity measure as the one used for mortality in SMR. Hence, first or second customization approaches could not be applied. 
12.1.3

Appendix A1.3 - List of Intensive care units

We calculated the standardized mortality ratio (SMR) and the standardized resource use (SMR), which composed the efficiency matrix in Table 1 (Manuscript). We provide the values of those indicators for each intensive care unit and its corresponding efficiency group classification in Table A11.2 The median SMR was 1.01 and the median SRU was 0.93. Units with both SMR and SRU lower than their respective medians were classified as efficient. The remaining units were considered non-efficient.

Table A12.2 - List of intensive care units and their performance indicators

\begin{tabular}{|c|c|c|c|c|c|c|c|c|c|c|c|}
\hline 3 & SMR & SRU & $\begin{array}{l}\text { Efficiency } \\
\text { Group }\end{array}$ & ICU & SMR & SRU & $\begin{array}{c}\text { Efficiency } \\
\text { Group }\end{array}$ & ICU & SMR & SRU & $\begin{array}{c}\text { Efficiency } \\
\text { Group }\end{array}$ \\
\hline 1 & 1.42 & 0.93 & Non-efficient & 41 & 1.19 & 1.26 & Non-efficient & 81 & 1.59 & 1.55 & Non-efficient \\
\hline 2 & 1.39 & 1.20 & Non-efficient & 42 & 1.36 & 2.07 & Non-efficient & 82 & 1.07 & 1.27 & Non-efficient \\
\hline 3 & 1.52 & 1.30 & Non-efficient & 43 & 1.42 & 2.24 & Non-efficient & 83 & 1.73 & 1.48 & Non-efficient \\
\hline 4 & 1.06 & 1.26 & Non-efficient & 44 & 0.61 & 1.19 & Non-efficient & 84 & 1.59 & 1.85 & Non-efficient \\
\hline 5 & 0.74 & 0.93 & Efficient & 45 & 0.75 & 0.76 & Efficient & 85 & 0.74 & 0.80 & Efficient \\
\hline 6 & 1.19 & 1.46 & Non-efficient & 46 & 0.63 & 0.74 & Efficient & 86 & 1.12 & 0.93 & Non-efficient \\
\hline 7 & 0.93 & 0.65 & Efficient & 47 & 0.62 & 0.44 & Efficient & 87 & 1.21 & 0.90 & Non-efficient \\
\hline 8 & 1.05 & 0.80 & Non-efficient & 48 & 1.24 & 1.29 & Non-efficient & 88 & 0.66 & 0.79 & Efficient \\
\hline 9 & 0.53 & 0.74 & Efficient & 49 & 0.97 & 1.04 & Non-efficient & 89 & 0.85 & 0.72 & Efficient \\
\hline 10 & 1.02 & 1.27 & Non-efficient & 50 & 0.43 & 0.57 & Efficient & 90 & 0.66 & 0.47 & Efficient \\
\hline 11 & 0.68 & 0.61 & Efficient & 51 & 1.42 & 2.14 & Non-efficient & 91 & 0.78 & 0.80 & Efficient \\
\hline 12 & 0.68 & 0.78 & Efficient & 52 & 1.52 & 1.70 & Non-efficient & 92 & 0.65 & 0.69 & Efficient \\
\hline 13 & 1.49 & 1.35 & Non-efficient & 53 & 0.91 & 0.71 & Efficient & 93 & 1.22 & 1.19 & Non-efficient \\
\hline 14 & 1.64 & 2.08 & Non-efficient & 54 & 0.64 & 0.87 & Efficient & 94 & 0.83 & 1.19 & Non-efficient \\
\hline 15 & 1.11 & 1.09 & Non-efficient & 55 & 0.62 & 0.74 & Efficient & 95 & 0.13 & 0.49 & Efficient \\
\hline 16 & 1.07 & 0.84 & Non-efficient & 56 & 0.74 & 0.82 & Efficient & 96 & 0.57 & 0.78 & Efficient \\
\hline 17 & 1.22 & 1.57 & Non-efficient & 57 & 1.27 & 1.83 & Non-efficient & 97 & 0.54 & 0.78 & Efficient \\
\hline 18 & 0.93 & 1.09 & Non-efficient & 58 & 1.00 & 1.14 & Non-efficient & 98 & 1.56 & 1.51 & Non-efficient \\
\hline 19 & 2.08 & 2.61 & Non-efficient & 59 & 0.43 & 0.47 & Efficient & 99 & 0.53 & 0.49 & Efficient \\
\hline 20 & 0.88 & 1.03 & Non-efficient & 60 & 0.95 & 0.71 & Efficient & 100 & 1.26 & 1.40 & Non-efficient \\
\hline 21 & 1.22 & 1.28 & Non-efficient & 61 & 0.70 & 0.67 & Efficient & 101 & 0.73 & 0.91 & Efficient \\
\hline 22 & 0.88 & 1.09 & Non-efficient & 62 & 1.29 & 0.87 & Non-efficient & 102 & 0.84 & 0.93 & Non-efficient \\
\hline 23 & 1.84 & 2.05 & Non-efficient & 63 & 0.72 & 0.94 & Non-efficient & 103 & 0.53 & 1.01 & Non-efficient \\
\hline 24 & 1.10 & 1.35 & Non-efficient & 64 & 0.56 & 0.46 & Efficient & 104 & 1.11 & 1.56 & Non-efficient \\
\hline 25 & 1.22 & 0.90 & Non-efficient & 65 & 0.70 & 0.69 & Efficient & 105 & 0.44 & 0.41 & Efficient \\
\hline 26 & 0.91 & 0.69 & Efficient & 66 & 1.03 & 0.94 & Non-efficient & 106 & 1.01 & 0.61 & Efficient \\
\hline 27 & 0.63 & 0.61 & Efficient & 67 & 0.83 & 0.80 & Efficient & 107 & 0.81 & 0.58 & Efficient \\
\hline 28 & 0.61 & 0.68 & Efficient & 68 & 1.02 & 0.87 & Non-efficient & 108 & 0.48 & 0.53 & Efficient \\
\hline 29 & 1.04 & 1.10 & Non-efficient & 69 & 1.29 & 2.32 & Non-efficient & 109 & 1.50 & 1.82 & Non-efficient \\
\hline 30 & 1.44 & 1.30 & Non-efficient & 70 & 2.03 & 1.74 & Non-efficient & 110 & 0.90 & 0.72 & Efficient \\
\hline 31 & 1.43 & 1.39 & Non-efficient & 71 & 1.07 & 1.39 & Non-efficient & 111 & 1.50 & 1.05 & Non-efficient \\
\hline 32 & 1.12 & 0.64 & Non-efficient & 72 & 0.92 & 1.85 & Non-efficient & 112 & 1.24 & 1.22 & Non-efficient \\
\hline 33 & 1.09 & 1.23 & Non-efficient & 73 & 0.89 & 0.84 & Efficient & 113 & 1.14 & 0.93 & Non-efficient \\
\hline 34 & 0.93 & 0.67 & Efficient & 74 & 1.19 & 1.39 & Non-efficient & 114 & 0.85 & 0.63 & Efficient \\
\hline 35 & 0.66 & 1.09 & Non-efficient & 75 & 1.33 & 1.03 & Non-efficient & 115 & 1.66 & 0.73 & Non-efficient \\
\hline 36 & 0.52 & 0.83 & Efficient & 76 & 0.77 & 0.82 & Efficient & 116 & 1.19 & 0.79 & Non-efficient \\
\hline 37 & 0.65 & 0.89 & Efficient & 77 & 1.29 & 1.50 & Non-efficient & 117 & 1.36 & 2.24 & Non-efficient \\
\hline 38 & 0.55 & 0.76 & Efficient & 78 & 1.53 & 1.39 & Non-efficient & 118 & 1.26 & 0.68 & Non-efficient \\
\hline 39 & 1.09 & 1.72 & Non-efficient & 79 & 0.96 & 0.84 & Efficient & & & & \\
\hline 40 & 1.17 & 1.43 & Non-efficient & 80 & 0.70 & 0.77 & Efficient & & & & \\
\hline
\end{tabular}

SMR: Standardized Mortality Ratio

SRU: Standardized Resource Use 


\subsection{4}

\section{Appendix A1.4 - Organizational characteristics}

Our study considered 63 characteristics of structure and process collected in the CHECKLIST-ICU trial. We provide the frequency of positive responses from ICUs for each characteristic. Fisher's exact test was considered due to the sample size (118 total possible positive responses) and provided the odds ratio and its respective 95\% Confidence Intervals. Results are shown in Table A11.3.

Table A12.3 - Descriptive statistics and univariate analysis of structure and process characteristics

\begin{tabular}{|c|c|c|c|c|}
\hline Structure and process & $\begin{array}{c}\text { Efficient } \\
n=47(\%)\end{array}$ & $\begin{array}{c}\text { Non-Efficient } \\
n=71(\%)\end{array}$ & OR $(95 \% \mathrm{Cl})$ & $\mathrm{p}$ \\
\hline \multicolumn{5}{|l|}{ (Care protocols) "Does the unit have/perform:" } \\
\hline Sepsis protocol & $27(57)$ & $31(44)$ & $1.73(0.78-3.93)$ & 0.19 \\
\hline Sedation protocol & $25(53)$ & $33(46)$ & $1.31(0.59-2.93)$ & 0.57 \\
\hline Analgesia protocol & $19(40)$ & $29(41)$ & $0.98(0.43-2.22)$ & 1.00 \\
\hline Ventilator Weaning protocol & $34(72)$ & $46(65)$ & $1.42(0.6-3.48)$ & 0.43 \\
\hline $\begin{array}{l}\text { Ventilator-Associated Pneumonia (VAP) prevention } \\
\text { protocol }\end{array}$ & $35(74)$ & $44(62)$ & $1.78(0.74-4.44)$ & 0.17 \\
\hline $\begin{array}{l}\text { Prevention of Central-Line Associated Bloodstream } \\
\text { infection (CLABSI) }\end{array}$ & $32(68)$ & $42(59)$ & $1.47(0.64-3.47)$ & 0.34 \\
\hline \multicolumn{5}{|l|}{ (Physical infrastructure) "Does the unit offer:" } \\
\hline Adult, Pediatric or Neonatal ICUs & $40(85)$ & $58(82)$ & $1.28(0.43-4.14)$ & 0.80 \\
\hline Privacy (curtain between beds) & $45(96)$ & $63(89)$ & $2.83(0.53-28.65)$ & 0.31 \\
\hline Insulation bed & $43(91)$ & $66(93)$ & $0.82(0.17-4.35)$ & 1.00 \\
\hline \multicolumn{5}{|l|}{ (Human resources) "Does the unit have:" } \\
\hline $\begin{array}{l}\text { Responsible technician with an intensive care } \\
\text { specialist title }\end{array}$ & - & - & - & - \\
\hline $\begin{array}{l}\text { Nursing coordinator is a specialist during intensive } \\
\text { care }\end{array}$ & - & - & - & - \\
\hline $\begin{array}{l}\text { Physiotherapist coordinator is a specialist during } \\
\text { intensive care }\end{array}$ & - & - & - & - \\
\hline $\begin{array}{l}\text { Routine physician per } 10 \text { beds or fraction in every } \\
\text { shift in the morning/evening }\end{array}$ & $45(96)$ & $66(93)$ & $1.7(0.26-18.55)$ & 0.70 \\
\hline $\begin{array}{l}\text { Exclusive routine physician per } 10 \text { beds or fraction } \\
\text { during every shift }\end{array}$ & $43(91)$ & $70(99)$ & $0.16(0-1.64)$ & $\underline{0.08}$ \\
\hline $\begin{array}{l}\text { Exclusive nurse per } 10 \text { beds or fraction during } \\
\text { every shift }\end{array}$ & - & - & - & - \\
\hline $\begin{array}{l}\text { Exclusive physiotherapist per } 10 \text { beds or fraction } \\
\text { during every shift }\end{array}$ & $46(98)$ & $69(97)$ & $1.33(0.07-80.31)$ & 1.00 \\
\hline $\begin{array}{l}\text { Exclusive nursing technician per } 10 \text { beds or fraction } \\
\text { during every shift }\end{array}$ & $41(87)$ & $66(93)$ & $0.52(0.12-2.19)$ & 0.34 \\
\hline Exclusive administrative assistant & $37(79)$ & $57(80)$ & $0.91(0.33-2.55)$ & 1.00 \\
\hline (Healthcare resources) "Does the unit provide:" & & & & - \\
\hline $\begin{array}{l}\text { Nutritional Assistance (with enteral and parenteral } \\
\text { nutrition) }\end{array}$ & - & - & - & - \\
\hline Nephrologic Assistance (with hemodialysis) & $45(96)$ & $69(97)$ & $0.65(0.05-9.33)$ & 1.00 \\
\hline Hemotherapic Assistance & - & - & - & - \\
\hline Infectology Clinical Assistance & $45(96)$ & $64(90)$ & $2.44(0.44-25.18)$ & 0.31 \\
\hline General Surgery Assistance & - & - & - & - \\
\hline $\begin{array}{l}\text { Clinical Laboratory service (including microbiology } \\
\text { and hemogasometry) }\end{array}$ & - & - & - & - \\
\hline Mobile Radiography service & - & - & - & - \\
\hline
\end{tabular}




\begin{tabular}{|c|c|c|c|c|}
\hline Portable Ultrasonography service & $42(89)$ & $64(90)$ & $0.92(0.23-3.93)$ & 1.00 \\
\hline Digestive Endoscopy (upper and lower) service & $44(94)$ & $65(92)$ & $1.35(0.27-8.78)$ & 1.00 \\
\hline Fiberoptic bronchoscopy service & $38(81)$ & $51(72)$ & $1.65(0.63-4.6)$ & 0.29 \\
\hline Surgical Center & $46(98)$ & $70(99)$ & $0.66(0.01-52.67)$ & 1.00 \\
\hline Echocardiography service & $45(96)$ & $67(94)$ & $1.34(0.18-15.41)$ & 1.00 \\
\hline Cardiovascular Surgery & $35(74)$ & $44(62)$ & $1.78(0.74-4.44)$ & 0.17 \\
\hline Neurologic Surgery & $37(79)$ & $59(83)$ & $0.75(0.27-2.16)$ & 0.63 \\
\hline Interventional Radiology & $32(68)$ & $47(66)$ & $1.09(0.46-2.6)$ & 1.00 \\
\hline Computer Tomography & $43(91)$ & $67(94)$ & $0.64(0.11-3.65)$ & 0.71 \\
\hline Confirmatory tests for brain blood flow & $34(72)$ & $49(69)$ & $1.17(0.49-2.91)$ & 0.84 \\
\hline
\end{tabular}

(Family policies) "Does the unit provide/permit:"

Orientation to relatives (at least once a day)

Visits of at least $30 \mathrm{~min}$

(Transport of patients) "Does the unit provide:"

Physician and nurse assistance in transportation of severe patients

$44(94) \quad 62(87) \quad 2.12(0.49-12.84) \quad 0.36$

Transportation of patient with adequate equipment

$32(68) \quad 60(85)$

$0.39(0.14-1.04) \quad \underline{0.04}$

(Risk management) "Does the unit have:"

\begin{tabular}{lllll} 
Adverse events recording routine & $32(68)$ & $37(52)$ & $1.95(0.85-4.59)$ & 0.09 \\
\hline $\begin{array}{l}\text { Presence of a person responsible to manage } \\
\text { adverse events }\end{array}$ & $26(55)$ & $34(48)$ & $1.34(0.6-3.02)$ & 0.46 \\
\hline
\end{tabular}

(Prevention of Healthcare-related infections) "Does the unit/Hospital Infection Control Committee:"

\begin{tabular}{|c|c|c|c|c|}
\hline $\begin{array}{l}\text { Research on infections related to invasive devices } \\
\text { and multi-resistance for clinical epidemiology }\end{array}$ & $46(98)$ & $64(90)$ & $4.98(0.61-231.35)$ & $\underline{0.14}$ \\
\hline Report results from infection surveillance & $40(85)$ & $53(75)$ & $1.93(0.69-6.02)$ & 0.25 \\
\hline Provide hand cleaning preparation at the unit & $46(98)$ & $68(96)$ & $2.02(0.16-108.76)$ & 1.00 \\
\hline \multicolumn{5}{|l|}{ (Quality-of-care metrics) "Does the unit evaluate:" } \\
\hline Absolute mortality rate & $42(89)$ & $56(79)$ & $2.24(0.7-8.5)$ & 0.21 \\
\hline $\begin{array}{l}\text { Expected Mortality rate estimation from severity } \\
\text { scores }\end{array}$ & $30(64)$ & $36(51)$ & $1.71(0.76-3.93)$ & 0.19 \\
\hline Average ICU Length of Stay (LOS) & $41(87)$ & $57(80)$ & $1.67(0.55-5.77)$ & 0.45 \\
\hline 24-hour readmission rate & $28(60)$ & $30(42)$ & $2(0.89-4.57)$ & $\underline{0.09}$ \\
\hline $\begin{array}{l}\text { Ventilator-Associated Pneumonia (VAP) incidence } \\
\text { density }\end{array}$ & $43(91)$ & $48(68)$ & $5.09(1.56-21.86)$ & $\underline{0.003}$ \\
\hline Mechanical Ventilation (MV) utilization rate & $40(85)$ & $44(62)$ & $3.47(1.29-10.51)$ & $\underline{0.01}$ \\
\hline $\begin{array}{l}\text { Central-Line Associated Bloodstream rate } \\
\text { (CLABSI) incidence density }\end{array}$ & $42(89)$ & $51(72)$ & $3.26(1.07-12.09)$ & $\underline{0.02}$ \\
\hline Central venous catheter (CVC) utilization rate & $41(87)$ & $42(59)$ & $4.66(1.67-15.2)$ & $\underline{0.001}$ \\
\hline Urinary Tract Infection (UTI) incidence density & $40(85)$ & $48(68)$ & $2.72(1-8.3)$ & $\underline{0.05}$ \\
\hline \multicolumn{5}{|l|}{ (Equipment) "Does the unit have:" } \\
\hline $\begin{array}{l}\text { Manual resuscitator with reservatory and facial } \\
\text { mask }\end{array}$ & $43(91)$ & $62(87)$ & $1.55(0.4-7.36)$ & 0.56 \\
\hline Infusion pump ( 4.3 per bed) & - & - & - & \\
\hline Multiparameter monitoring & - & - & - & \\
\hline "Cuffometer" & $41(87)$ & $54(76)$ & $2.14(0.72-7.23)$ & 0.16 \\
\hline Mechanical Ventilator (one unit for each two beds) & $45(96)$ & $70(99)$ & $0.32(0.01-6.4)$ & 0.56 \\
\hline $\begin{array}{l}\text { Non-invasive mechanical ventilator (one for each } \\
\text { ten beds) }\end{array}$ & $43(91)$ & $61(86)$ & $1.75(0.47-8.17)$ & 0.40 \\
\hline Portable Electrocardiogram (one for each ten beds) & $43(91)$ & $67(94)$ & $0.64(0.11-3.65)$ & 0.71 \\
\hline $\begin{array}{l}\text { Defibrillator/cardioverter kit (one for each five beds } \\
\text { or fraction) }\end{array}$ & $39(83)$ & $54(76)$ & $1.53(0.56-4.53)$ & 0.49 \\
\hline $\begin{array}{l}\text { Temporary cardiac pacemaker, electrodes and } \\
\text { generator (one equipment for each ten beds) }\end{array}$ & $45(96)$ & $63(89)$ & $2.83(0.53-28.65)$ & 0.31 \\
\hline
\end{tabular}




\begin{tabular}{l} 
Refrigerator with temperature control \\
\hline OR $(95 \% \mathrm{Cl})$ : Odds ratio (95\% confidence interval) \\
" - ": Odds ratio and $95 \% \mathrm{Cl}$ not available since proportion is $100 \%$ in both groups \\
"Not applicable to all units \\
Underscored: selected variables for the analysis
\end{tabular}

For this analysis, we evaluated only the items applicable to all the units and in which there was at least one positive response. Hence, from 63 organizational characteristics, 50 were eligible, and 10 were selected for the multivariable analysis.

\subsection{5}

\section{Appendix A1.5 - Adaptive elastic-net hyperparameter estimation and confidence intervals}

Regularization consists of adding a penalization parameter to the conventional regression coefficients, improving the prediction accuracy, including a large number of predictors, even when the number of variables is higher than the sample size, and manages multicollinearity since it can group the correlated variables (BARRETT; LOCKHART, 2019; HASTIE; TIBSHIRANI; FRIEDMAN, 2009).

The adaptive elastic-net regularization (AENet) method is a regularization method based on the elastic-net regularization in which L1 and L2 norm penalties are added to estimates/coefficients to reduce their variance with the application of bias. However, to diminish the bias, AENet considers adaptive weights to coefficients and, therefore, can provide the oracle properties of its estimator (ZOU; ZHANG, 2009). Therefore, we chose to perform a multivariable analysis using the AENET model. We used the msaenet (XIAO; XU, 2015) and glmnet (FRIEDMAN; HASTIE; TIBSHIRANI, 2010) R packages to fit the AENet to the logistic regression.

The AENet estimator is:

$$
\widehat{\boldsymbol{\beta}}(A E N e t)=\left(1+\frac{\lambda_{2}}{n}\right)\left\{\operatorname{argmin}_{\beta}\|\boldsymbol{y}-\boldsymbol{X} \boldsymbol{\beta}\|_{2}^{2}+\lambda_{2}\|\beta\|_{2}^{2}+\lambda_{1} \sum_{j=1}^{p} \widehat{w}_{j}\left|\beta_{j}\right|\right\}(1),
$$

in which:

$\widehat{\boldsymbol{\beta}}(A E N e t)$ : the vector of the estimated coefficients

$\lambda_{1}$ and $\lambda_{2}$ : penalty factors for the L1 and L2 norms, respectively

$\widehat{w}_{j}$ : adaptive weights, defined as $\widehat{w}_{j}=(\mid \hat{\beta}(\text { ENet }) \mid)^{-\gamma}$, which consists of the scaled coefficients from an elastic-net or ridge regularization initially fitted to the data, where $\gamma$ is the scaling factor;

$y$ : vector of the response values $(n \times 1)$ 
$X$ : matrix of the predictor values $(n \times p)$

One approach derived from the elastic-net (ZOU; HASTIE, 2005) is to combine $\lambda_{1}$ and $\lambda_{2}$ into one hyperparameter $\alpha=\frac{\lambda_{2}}{\lambda_{1}+\lambda_{2}}$, providing $(1-\alpha)\|\beta\|_{2}^{2}+\alpha|\beta|$ and $\lambda_{2}=\lambda$. Hence, if $\alpha \rightarrow 0$, the model converges to have only the L2 norm, called the Ridge regression, whereas if $\alpha \rightarrow 1$, the L1 norm is evident, called LASSO. In our study, we considered this transformation, since it is the default implementation in the msaenet (XIAO; XU, 2015) and glmnet (FRIEDMAN; HASTIE; TIBSHIRANI, 2010) packages.

Following the method's procedure, we considered the ridge regularization estimates as the initial coefficients since all variables would have initial weights for AENet. We then estimated three hyperparameters: the penalty $\lambda, \alpha$, and $\gamma$.

To estimate the scaling factor, we followed the definition of a fixed $\gamma$ provided by Zou and Hang (ZOU; ZHANG, 2009): $\gamma=\left\lceil\frac{2 v}{1-v}\right\rceil+1$, where $\lim _{n \rightarrow \infty} \frac{\ln (p)}{\ln (n)}=v$ when $n>p$, where $n$ is the number of observations and $p$ is the number of variables. In our study, for the multivariable analysis, there were 10 variables (organizational characteristics) and 118 observations, which resulted in $v \cong 0.4826$ and therefore $\gamma=3$.

The hyperparameters $\lambda$ and $\alpha$ were then estimated using a 10-fold cross-validation and testing the combination of sequences for $\gamma=3$. For $\lambda$, we considered the default sequence generated in the glmnet package, which is estimated using the data matrix; for $\alpha$, we assessed the range from 0.01 to 0.99 . The final values were those that provided the minimum cross-validation classification error: $\lambda=3969.911$ and $\alpha=0.01$. A summary of the hyperparameter estimation is shown in Table A11.4.

Table A12.4 - Intervals and final results of the AENet hyperparameter estimation

\begin{tabular}{ccc}
\hline Hyperparameter & Definition/tested interval & Best values \\
\hline$\gamma$ & $\gamma=\left[\frac{2 v}{1-v}\right]+1$ & 3 \\
$\lambda$ & Default glmnet package & \\
$\alpha$ & lambda sequence & 3969.911 \\
& {$[0.01,0.99]$} & 0.01 \\
\hline
\end{tabular}

We applied the AENet regularization in the multivariable logistic regression and obtained the estimates as odds ratios (OR) for each variable. Due to the selection feature 
of the model provided by the L1 norm as $\alpha>0$, some variables had $\mathrm{OR}=1.00(\log (\mathrm{OR})$ $=0$ ), which means that the coefficients for those variables was shrunk.

For the same model (fixed values of hyperparameters), we generated basic bootstrap confidence intervals after 10,000 resamples. The lower and upper confidence limits were estimated as $\left(2 \hat{\beta}(A E N e t)-\beta^{\text {Boot }}{ }_{.975}, 2 \hat{\beta}(A E N e t)-\beta^{\text {Boot }}{ }_{.025}\right)$, where $\hat{\beta}(A E N e t)$ are the estimated coefficients from the AENet logistic regression model. $\beta_{.025}^{\text {Boot }}$ and $\beta_{.975}^{\text {Boot }}$ are the percentiles 2.5 and 97.5 , respectively, from the bootstrapped distribution of the coefficients.

As Ridge, LASSO, or Elastic Net estimators are naturally biased their confidence intervals would be imprecise. The literature has shown statistical bias corrections and confidence intervals based both in bootstrapping/resampling procedures or asymptotic tests for penalized regressions, especially LASSO (CHATTERJEE; LAHIRI, 2011; LOCKHART et al., 2014). However, as far as the authors know, there are no such procedures for the ANET, and its estimator has oracle properties. Also, bootstrapping is a non-parametric simulation method that does not rely on strict assumptions for the coefficients' distribution during inference (i.e., assuming normality or t-student) (CARPENTER; BITHELL, 2000). Hence, we provide bootstrapped confidence intervals for AENET. We are aware of the limitations when using bootstrapping, and that new assumptions on AENET coefficients can change the estimated intervals.

For reproducibility of those results, our random number generator's seed was $2^{\wedge} 31$ $1(2147483647)$ 
12.2

Appendix A2

12.2.1

Appendix A2.1 - Calibration of SAPS-3 and APACHE-IV mortality risk models
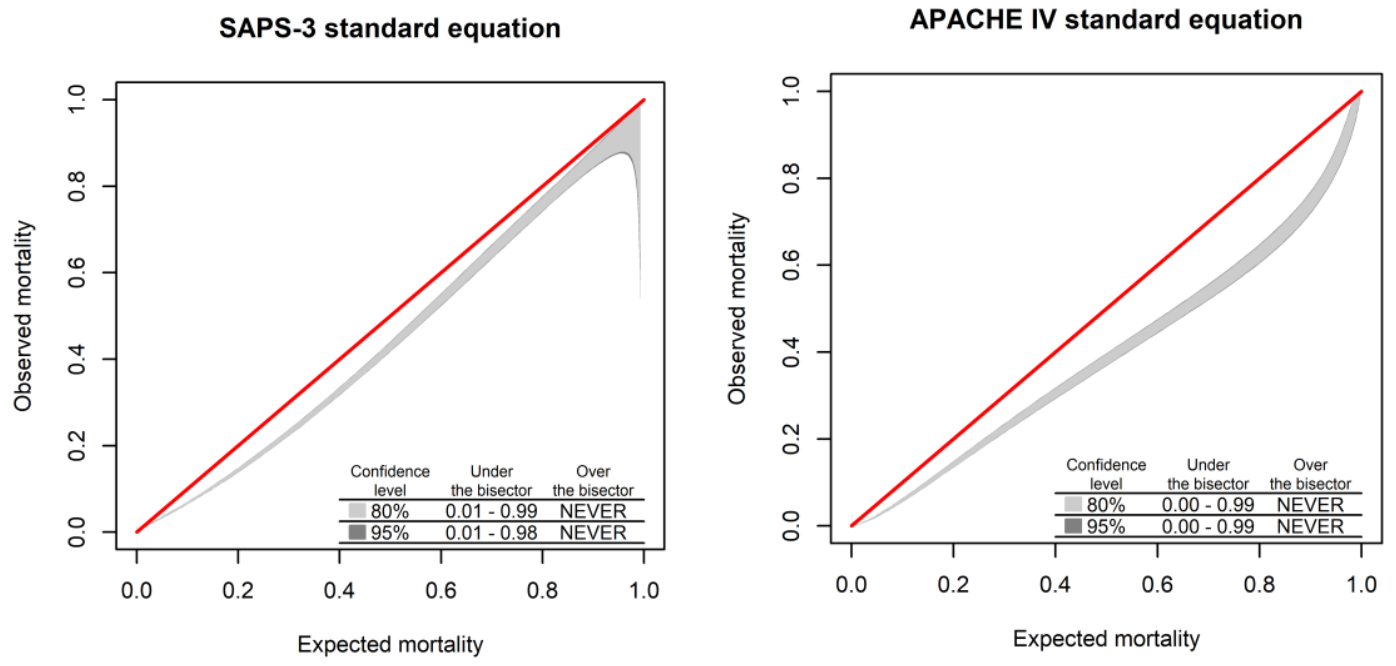

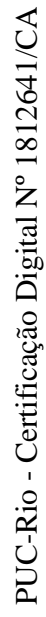
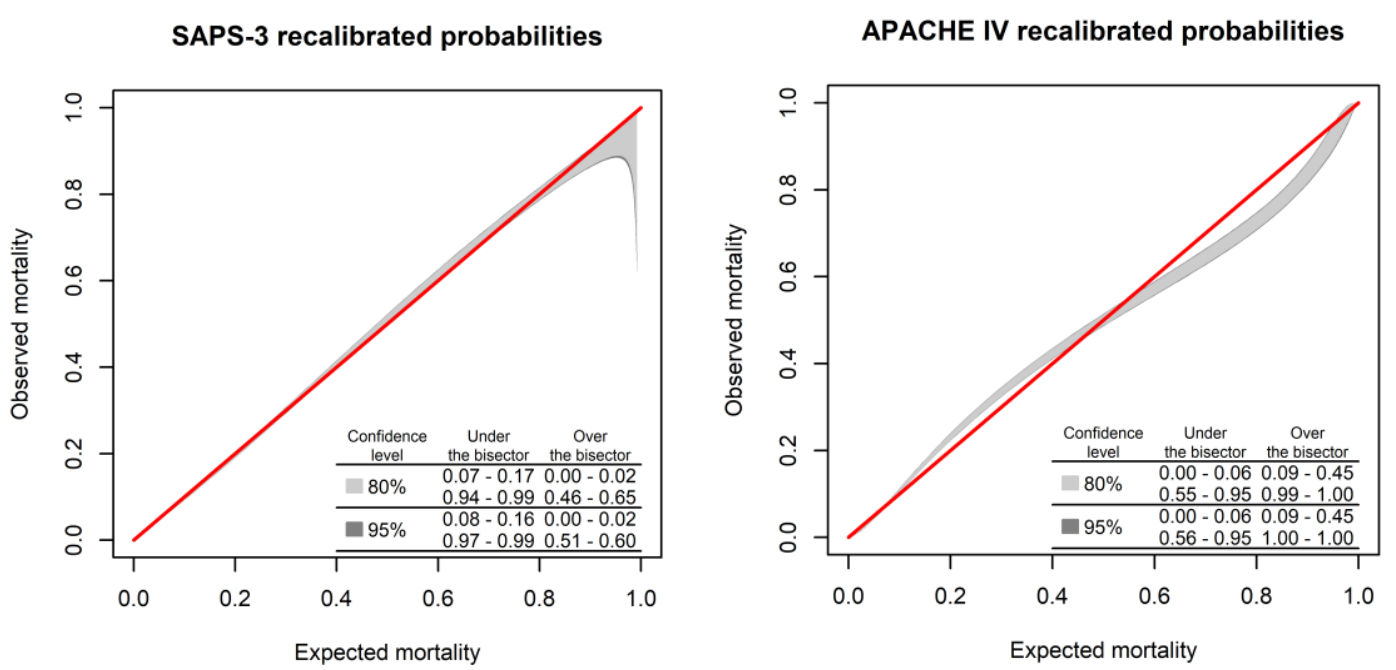
12.2.2

Appendix A2.2 - Average length of stay for each SAPS-3 and APACHE-IV decile

\begin{tabular}{|c|c|c|c|c|c|c|c|c|c|}
\hline \multirow{3}{*}{ Decile } & \multirow{3}{*}{$\begin{array}{l}\text { Probabillity } \\
\text { Range* }^{*}\end{array}$} & \multicolumn{4}{|c|}{ Brazil (SAPS-3) } & \multicolumn{4}{|c|}{ The Netherlands (APACHE-IV) } \\
\hline & & \multicolumn{2}{|c|}{$\begin{array}{c}\text { Number of ICU } \\
\text { patients }\end{array}$} & \multicolumn{2}{|l|}{$\begin{array}{l}\text { ICU LOS } \\
\text { (days) }\end{array}$} & \multicolumn{2}{|c|}{$\begin{array}{l}\text { Number of ICU } \\
\text { patients }\end{array}$} & \multicolumn{2}{|c|}{ ICU LOS (days) } \\
\hline & & Total & Survivors & Total & Per survivor & Total & Survivors & Total & Per survivor \\
\hline 1 & {$[0.0,0.1]$} & 172,186 & 166,297 & 568,902 & 3.4 & 106,858 & 103,879 & 326,279 & 3.1 \\
\hline 2 & ] $0.1,0.2]$ & 48,840 & 41,980 & 278,053 & 6.6 & 22,449 & 18,805 & 128,637 & 6.8 \\
\hline 3 & ] $0.2,0.3]$ & 20,790 & 15,763 & 146,068 & 9.3 & 10,467 & 7,536 & 72,230 & 9.6 \\
\hline 4 & ] $0.3,0.4]$ & 13,502 & 8,922 & 110,661 & 12.4 & 6,657 & 4,077 & 49,518 & 12.1 \\
\hline 5 & ] $0.4,0.5]$ & 8,665 & 4,672 & 80,900 & 17.3 & 4,663 & 2,549 & 34,242 & 13.4 \\
\hline 6 & ] $0.5,0.6]$ & 5,968 & 2,614 & 58,461 & 22.4 & 3,813 & 1,736 & 28,102 & 16.2 \\
\hline 7 & ] $0.6,0.7]$ & 4,940 & 1,679 & 47,833 & 28.5 & 3,215 & 1,284 & 23,975 & 18.7 \\
\hline 8 & ] $0.7,0.8]$ & 3,627 & 920 & 35,339 & 38.4 & 2,745 & 921 & 18,429 & 20.0 \\
\hline 9 & ] $0.8,0.9]$ & 2,932 & 481 & 26,498 & 55.1 & 2,178 & 469 & 12,922 & 27.6 \\
\hline 10 & ] $0.9,1.0]$ & 853 & 77 & 5,367 & 69.7 & 1,354 & 120 & 6,247 & 52.1 \\
\hline
\end{tabular}

*Probabilities were estimated using recalibrated models from SAPS-3 (Brazil) and APACHE-IV (The Netherlands) units 
12.2.3

Appendix A2.3 - Distribution of performance metrics (SMR, SRU and ASER) per units stratified by efficiency groups

\begin{tabular}{|c|c|c|c|c|c|}
\hline Metrics & Overall & Most Efficient & Underachieving & Overachieving & Least Efficient \\
\hline \multicolumn{6}{|l|}{ Brazil } \\
\hline No. Of ICUs & 134 & 47 (35\%) & $20(15 \%)$ & $20(15 \%)$ & 47 (35\%) \\
\hline \multicolumn{6}{|l|}{ SMR } \\
\hline Median (IQR) & $0.97(0.75,1.21)$ & $0.72(0.67,0.84)$ & $1.06(1.01,1.22)$ & $0.80(0.74,0.86)$ & $1.25(1.16,1.47)$ \\
\hline Mean (SD) & $1.03(0.36)$ & $0.75(0.12)$ & $1.12(0.14)$ & $0.79(0.11)$ & $1.37(0.34)$ \\
\hline \multicolumn{6}{|l|}{ SRU } \\
\hline Median (IQR) & $1.06(0.79,1.34)$ & $0.75(0.66,0.90)$ & $0.86(0.78,0.96)$ & $1.22(1.10,1.38)$ & $1.41(1.21,1.67)$ \\
\hline Mean (SD) & $1.15(0.58)$ & $0.77(0.16)$ & $0.85(0.14)$ & $1.27(0.21)$ & $1.60(0.74)$ \\
\hline \multicolumn{6}{|l|}{ ASER } \\
\hline Median (IQR) & $0.98(0.81,1.23)$ & $0.78(0.70,0.82)$ & $0.98(0.93,1.02)$ & $0.99(0.95,1.12)$ & $1.39(1.18,1.55)$ \\
\hline Mean (SD) & $1.09(0.44)$ & $0.76(0.10)$ & $0.98(0.12)$ & $1.03(0.13)$ & $1.48(0.50)$ \\
\hline \multicolumn{6}{|l|}{ The Netherlands } \\
\hline No. Of ICUs & 83 & $25(30 \%)$ & 17 (20.5\%) & $17(20.5 \%)$ & 24 (29\%) \\
\hline \multicolumn{6}{|l|}{ SMR } \\
\hline Median (IQR) & $1(0.89,1.12)$ & $0.86(0.79,0.95)$ & $1.14(1.12,1.27)$ & $0.89(0.84,0.92)$ & $1.08(1.05,1.15)$ \\
\hline Mean (SD) & $1(0.18)$ & $0.85(0.13)$ & $1.19(0.09)$ & $0.87(0.09)$ & $1.11(0.08)$ \\
\hline \multicolumn{6}{|l|}{ SRU } \\
\hline Median (IQR) & $0.98(0.88,1.08)$ & $0.87(0.79,0.92)$ & $0.9(0.85,0.94)$ & $1.07(1.02,1.08)$ & $1.12(1.04,1.17)$ \\
\hline Mean (SD) & $0.99(0.16)$ & $0.85(0.1)$ & $0.89(0.07)$ & $1.07(0.08)$ & $1.14(0.14)$ \\
\hline \multicolumn{6}{|l|}{ ASER } \\
\hline Median (IQR) & $0.99(0.92,1.09)$ & $0.87(0.78,0.92)$ & $1.03(0.99,1.08)$ & $0.97(0.95,1)$ & $1.11(1.07,1.17)$ \\
\hline Mean (SD) & $0.99(0.14)$ & $0.85(0.11)$ & $1.04(0.07)$ & $0.97(0.06)$ & $1.12(0.08)$ \\
\hline
\end{tabular}

ASER: Average Standardized Efficiency Ratio; ICU: Intensive Care Unit; IQR: Interquartile range; SD: Standard Deviation; SMR: Standardized Mortality Ratio; SRU: Standardized Resource USE 


\section{2 .4}

\section{Appendix A2.4 - Distribution of SMR, SRU and ASER values per country}

(A) Brazil

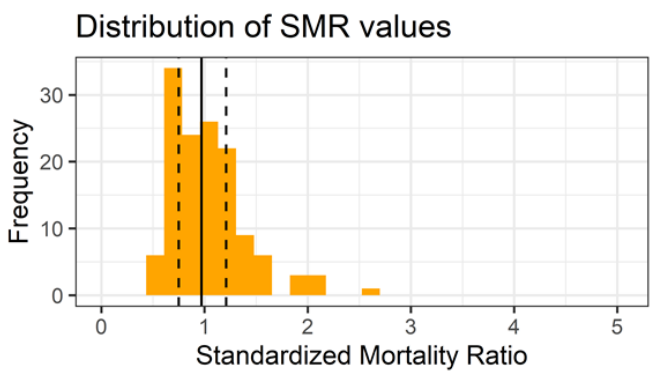

Distribution of SRU values

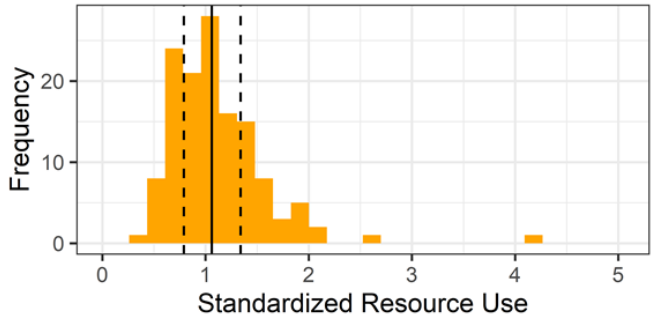

Distribution of ASER values

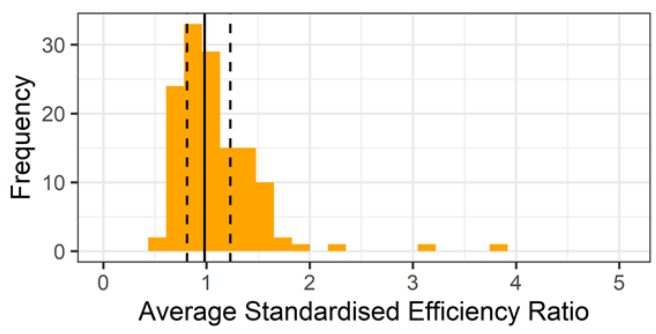

(B) The Netherlands

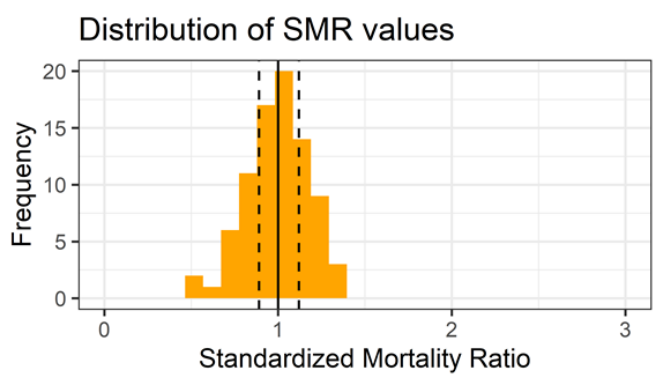

Distribution of SRU values

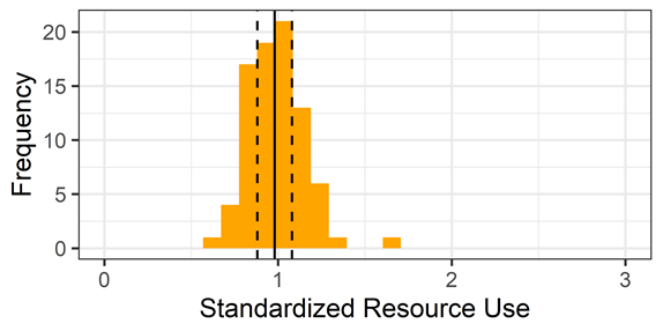

Distribution of ASER values

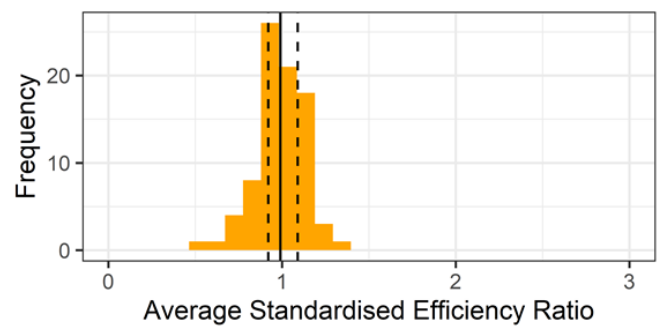


12.3

\section{Appendix A4}

12.3.1

Appendix A4.1 - Data Coverage, data sources and variables

Table A12.5 - Database coverage in terms of Brazilian municipalities and population

\begin{tabular}{|c|c|c|c|c|c|c|}
\hline & Brazil & North & Northeast & Central-West & Southeast & South \\
\hline \multicolumn{7}{|l|}{ Municipality coverage } \\
\hline Municipalities & 5,570 & 450 & 1,794 & 467 & 1,668 & 1,191 \\
\hline Municipalities with COVID-19 case & $\begin{array}{c}5,506 \\
(98.9 \%)\end{array}$ & $\begin{array}{c}450 \\
(100 \%)\end{array}$ & $\begin{array}{c}1787 \\
(99.6 \%)\end{array}$ & $\begin{array}{c}462 \\
(98.9 \%)\end{array}$ & $\begin{array}{c}1,637 \\
(98.1 \%)\end{array}$ & $1,170(98.2 \%)$ \\
\hline $\begin{array}{l}\text { Municipalities with at least } 1 \text { hospitalised } \\
\text { patient in SIVEP-Gripe }\end{array}$ & $\begin{array}{c}4407 / 5506 \\
(80 \%)\end{array}$ & $\begin{array}{c}369 / 450 \\
(82 \%)\end{array}$ & $\begin{array}{c}1378 / 1787 \\
(77 \%)\end{array}$ & $\begin{array}{c}368 / 462 \\
(80 \%)\end{array}$ & $\begin{array}{c}1347 / 1637 \\
(82 \%)\end{array}$ & $\begin{array}{c}945 / 1170 \\
(81 \%)\end{array}$ \\
\hline \multicolumn{7}{|l|}{ Population coverage } \\
\hline Total population & $211,755,692$ & $18,672,591$ & $57,374,243$ & $16,504,303$ & $89,012,240$ & $30,192,315$ \\
\hline $\begin{array}{l}\text { Total population from municipalities that } \\
\text { reported } 1 \text { adult hospitalised case in SIVEP- } \\
\text { Gripe }\end{array}$ & $\begin{array}{l}203,250,793 \\
(96 \%)\end{array}$ & $\begin{array}{c}17,934,414 \\
(96 \%)\end{array}$ & $\begin{array}{l}53,253,926 \\
(93 \%)\end{array}$ & $\begin{array}{l}15,933,545 \\
(97 \%)\end{array}$ & $\begin{array}{c}87,072,377 \\
\quad(98 \%)\end{array}$ & $\begin{array}{l}29,056,531 \\
(96 \%)\end{array}$ \\
\hline
\end{tabular}

Brazilian population based on the 2020 projections. 
Table A12.6 - Description of the data sources used in this study

\begin{tabular}{|c|c|c|c|c|}
\hline Data & Source & Source address & Version & Date exported \\
\hline $\begin{array}{l}\text { COVID-19 } \\
\text { hospitalized } \\
\text { cases }\end{array}$ & $\begin{array}{l}\text { Influenza Epidemiological Surveillance } \\
\text { Information System, "SIVEP-Gripe" }\end{array}$ & $\begin{array}{l}\text { OpenDataSUS repository: } \\
\text { https://opendatasus.saude.gov.br/dataset/bd-sraq-2020 } \\
\text { Direct link for data of 12/10/2020: } \\
\text { https://s3-sa-east-1.amazonaws.com/ckan.saude.gov.br/SRAG/2020//NFLUD-12-10- } \\
\underline{2020 . \text { csv }}\end{array}$ & $12 / 10 / 2020$ & 14/10/2020 \\
\hline $\begin{array}{l}\text { COVID- } 19 \text { cases } \\
\text { and deaths by } \\
\text { municipalities }\end{array}$ & $\begin{array}{l}\text { State health departments ("SES"); } \\
\text { Extracted and validated by brasil.io }\end{array}$ & https://brasil.io/dataset/covid19/caso full/ & 20/10/2020 & 20/10/2020 \\
\hline $\begin{array}{l}\text { Brazilian } \\
\text { population } \\
\text { dataset }\end{array}$ & $\begin{array}{l}\text { Brazilian Institute of Geography and } \\
\text { Statistics, "IBGE" }\end{array}$ & $\begin{array}{l}\text { Official IBGE website: } \\
\text { https://www.ibge.gov.br/home/ } \\
\text { Direct link for population projection: } \\
\text { ftp://ftp.ibge.gov.br/Projecao da Populacao/Projecao da Populacao 2018/projecoe } \\
\text { s 2018 populacao } 2010206020200406 . x 1 \mathrm{~s}\end{array}$ & 06/04/2020 & $13 / 08 / 2020$ \\
\hline $\begin{array}{l}\text { Hospital and } \\
\text { ICU beds }\end{array}$ & $\begin{array}{l}\text { National Registry of Health } \\
\text { Establishments, "CNES" }\end{array}$ & $\begin{array}{l}\text { Official CNES website: } \\
\text { http://cnes.datasus.gov.br/ } \\
\text { Direct link for data: } \\
\text { ftp://ftp.datasus.gov.br/cnes/BASE DE DADOS CNES 202002.ZIP }\end{array}$ & $11 / 03 / 2020$ & $13 / 08 / 2020$ \\
\hline
\end{tabular}


Table A 12.7 - Detailed description of variables used in the study

\begin{tabular}{|c|c|c|c|c|c|}
\hline $\begin{array}{l}\text { Original variable } \\
\text { name }\end{array}$ & Variable & $\begin{array}{l}\text { Original } \\
\text { coding }\end{array}$ & Collected from & Coding for this study & Comments \\
\hline CS_SEXO & Sex & 3 levels & National ID & $\begin{array}{l}\text { Recoded to } 2 \text { levels. The lgnored level was considered as } \\
\text { missing }\end{array}$ & \\
\hline $\begin{array}{l}\text { NU_IDADE_N } \\
\text { \& TP_IDADE }\end{array}$ & Age & Integer & $\begin{array}{l}\text { Derived from difference } \\
\text { between birth date and } \\
\text { first symptoms date }\end{array}$ & Recoded to 6 levels & \\
\hline CS_RACA & $\begin{array}{l}\text { Self-reported race or } \\
\text { skin colour }\end{array}$ & 6 levels & Self-reported & $\begin{array}{l}\text { Recoded to } 4 \text { levels: combined Black and Brown; kept } \\
\text { White, Asian and Indigenous. The Ignored level was } \\
\text { considered as missing }\end{array}$ & $\begin{array}{l}\text { Self-reported race or skin } \\
\text { colour is an important } \\
\text { surrogate for socioeconomic } \\
\text { position, } \\
\text { social inequality, social capital } \\
\text { and structural racism in Brazil. } \\
\text { We referred to as "self- } \\
\text { reported race"a }\end{array}$ \\
\hline CS_ESCOL_N & Level of Education & 7 levels & Self-reported & $\begin{array}{l}\text { Recoded to } 4 \text { levels. Collapsing the categories of } \\
\text { intermediate levels of education in "Up to high school" } \\
\text { (included middle and elementary school). The Ignored level } \\
\text { was considered as missing }\end{array}$ & Recoded to avoid sparse data \\
\hline SG_UF_INTE & Region & 27 levels & $\begin{array}{l}\text { State of hospital } \\
\text { admission }\end{array}$ & $\begin{array}{l}\text { Recoded to } 5 \text { levels according to the official } 5 \text { geopolitical } \\
\text { regions of Brazil. }\end{array}$ & \\
\hline UTI & ICU admission & 3 levels & Clinical record & $\begin{array}{l}\text { Recoded to } 2 \text { levels. The Ignored level was considered as } \\
\text { missing }\end{array}$ & \\
\hline SUPORT_VEN & Respiratory support & 4 levels & Clinical record & $\begin{array}{l}\text { Recoded to } 3 \text { levels. The Ignored level was considered as } \\
\text { missing }\end{array}$ & \\
\hline $\begin{array}{l}\text { FEBRE, TOSSE, } \\
\text { GARGANTA, } \\
\text { DISPNEIA, } \\
\text { DESC_RESP, } \\
\text { SATURACAO, }\end{array}$ & Symptoms & 3 levels & $\begin{array}{l}\text { Clinical record/Self- } \\
\text { reported }\end{array}$ & $\begin{array}{l}\text { Recoded to } 2 \text { levels. The Ignored level was considered as } \\
\text { missing }\end{array}$ & \\
\hline
\end{tabular}


DIARREIA, VOMITO,

OUTRO_SIN

CARDIOPATI,

HEMATOLOG

HEPATICA,

DIABETES,

NEUROLOGIC,

PNEUMOPATI,

Comorbidities 3 levels Clinical record/Self

reported

Recoded to 3 levels. Missing and ignored levels were

RENAL, OBESIDA

considered as no comorbidity

IMUNODEPRE,

ASMA, SIND_DOWN

PUERPERA,

OUT_MORB

DT_NOTIFIC,

DT_SIN_PRI,

DT_INTERRA,

DT_ENTUTI,

Dates

Not applicable

User entered / notification

We checked dates and corrected those typos in YYYY

DT_SAIDUTI,

DT_EVOLUCA

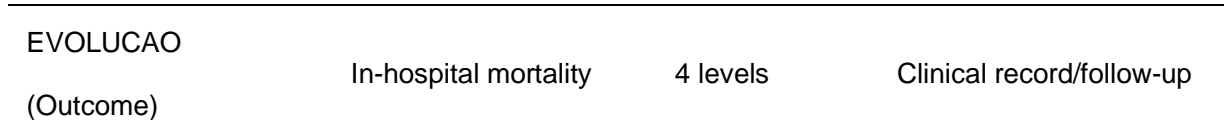

Recoded to 2 levels (Death/Discharge). Deaths grouped as

all-cause in-hospital mortality. Missing and ignored levels

were considered as missing and not use in the main

analysis.

We derived ICU mortality for those patients who were

admitted to the ICU and have available both ICU and

Not applicable ICU mortality Not applicable Derived hospital discharge dates. We considered ICU death when

the patient died in the hospital and had the same date for

ICU and hospital discharge.

Derived variable by considering any comorbidity

\begin{tabular}{|c|c|c|c|c|c|}
\hline Not applicable & Any comorbidity & Not applicable & Derived & $\begin{array}{l}\text { (Cardiovascular disease, Diabetes, Kidney disease, Obesity, } \\
\text { Neurological disease, Chronic obstructive pulmonary } \\
\text { disease, Immunodepression, Haematological disease, and } \\
\text { Hepatic disease) }\end{array}$ & $\begin{array}{l}\text { Selected by the literature of } \\
\text { comorbidities associated with } \\
\text { poor outcomes in COVID-19 }\end{array}$ \\
\hline Not applicable & $\begin{array}{l}\text { Number of } \\
\text { comorbidities }\end{array}$ & Not applicable & Derived & $\begin{array}{l}\text { We added the nine comorbidities above for those patients } \\
\text { without missing data in any of them in complete-case } \\
\text { analysis }\end{array}$ & \\
\hline
\end{tabular}




\begin{tabular}{|c|c|c|c|c|c|}
\hline Not applicable & $\begin{array}{l}\text { SARI (severe acute } \\
\text { respiratory infection) }\end{array}$ & Not applicable & Derived & $\begin{array}{l}\text { Combination of symptoms: High fever }\left(>37.8^{\circ} \mathrm{C}\right) \text { AND } \\
\text { [Cough OR Sore Throat] AND [Respiratory distress OR } \\
\text { Dyspnoea OR Oxygen saturation }<95 \% \text { ] }\end{array}$ & $\begin{array}{l}\text { Original SARI definition also } \\
\text { considers deaths outside } \\
\text { hospitals. We derived SARI for } \\
\text { those hospitalized. }\end{array}$ \\
\hline Not applicable & $\begin{array}{l}\text { SARI (severe acute } \\
\text { respiratory infection) } \\
\text { without fever criterion }\end{array}$ & Not applicable & Derived & $\begin{array}{l}\text { Combination of symptoms: [Cough OR Sore Throat] AND } \\
\text { [Respiratory distress OR Dyspnoea OR Oxygen saturation < } \\
\text { 95\%] }\end{array}$ & $\begin{array}{l}\text { Adapted SARI definition for } \\
\text { COVID-19 over the pandemic. } \\
\text { We derived SARI for those } \\
\text { hospitalized. }\end{array}$ \\
\hline Not applicable & $\begin{array}{l}\text { Time from onset of } \\
\text { symptoms to Hospital } \\
\text { admission, to ICU } \\
\text { admission, and to } \\
\text { death }\end{array}$ & Not applicable & Derived & $\begin{array}{l}\text { We derived times from the date of the first symptoms to the } \\
\text { date of hospital admission, ICU admission, and to death, } \\
\text { when the dates are available. Times are in days. We } \\
\text { censored the times in } 30 \text { days (percentile 95) and } \\
\text { considered } 0 \text { days as missing. }\end{array}$ & \\
\hline Not applicable & $\begin{array}{l}\text { Length-of-stay in the } \\
\text { Hospital and in the ICU }\end{array}$ & Not applicable & Derived & $\begin{array}{l}\text { Length-of-stay were calculated in days using the reported } \\
\text { dates of admission and discharge of hospital or the ICU. }\end{array}$ & \\
\hline $\begin{array}{l}\text { PCR_SARS2, } \\
\text { PCR_RESUL, } \\
\text { DS_PCR_OUT }\end{array}$ & $\begin{array}{l}\text { RT-qPCR status for } \\
\text { SARS-CoV-2 }\end{array}$ & Not applicable & Derived & $\begin{array}{l}\text { We corrected few patients that had positive RT-qPCR for } \\
\text { SARS-CoV-2 but it was described as string in } \\
\text { DS_PCR_OUT }\end{array}$ & $\begin{array}{l}\text { Ministry of Health } \\
\text { recommendation }\end{array}$ \\
\hline
\end{tabular}

${ }^{\mathrm{a}}$ Addressing racial inequalities in a pandemic: data limitations and a call for critical analyses. Pilecco FB, Leite L, Góes EF, Diele-Viegas LM, Aquino EML. Lancet Glob Health. 2020 Sep 15:S2214109X(20)30360-0. /

The correlation between ancestry and color in two cities of Northeast Brazil with contrasting ethnic compositions. Magalhaes da Silva T, Sandhya Rani MR, de Oliveira Costa GN, et al. Eur J Hum Genet 2015 ; 23(7): 984-9 


\section{Appendix A4.2 - Multiple imputation procedure and results}

To conduct the multiple imputation, we used the database with a defined hospital outcome $(n=232,036)$. We first investigated the patterns of missing variables. We explored whether missing values were conditioned on observed variables and the pattern suggested a missing at random (MAR) mechanism (Sterne JA, White IR, Carlin JB, et al. Multiple imputation for missing data in epidemiological and clinical research: potential and pitfalls. BMJ 2009; 338: b2393) (eTables 4, 5, 6 and 7 and eFigure 1). We conducted multiple imputation by chained equations using the command mi impute in Stata $13.1 \mathrm{We}$ followed the recommended steps to build the imputed model, including all variables of the interest, auxiliary variables (temporality: week of symptoms onset, regional: region/hospitalization in capitals, age) and the outcome. Below we show the imputation model specification and specifies the method used for each imputed variable. We generated 30 imputed datasets, following recent recommendations (Madley-Dowd P, et al. J Clin Epidemiol. 2019, 110:63-73) on and combined the results using Rubin's rule (Rubin DB. Multiple imputation for nonresponse in surveys. New York;: Wiley; 1987.). The distribution of the imputed variables before-and-after the imputation in on eTable 8. We also checked the convergence of values following the iterative process (10 iterations).

\begin{tabular}{|c|c|}
\hline Variable & \\
\hline Imputed variables & $\begin{array}{l}\text { Sex, self-reported race, ICU admission, } \\
\text { Respiratory support, Comorbidities } \\
\text { (Cardiovascular disease, Diabetes, Kidney disease, } \\
\text { Obesity, Neurological disease, Chronic obstructive } \\
\text { pulmonary disease, Immunodepression, } \\
\text { Haematological disease, and Hepatic disease), SARI, } \\
\text { Oxygen saturation <95\%, Dyspnoea, Respiratory } \\
\text { distress and time from symptoms onset to } \\
\text { hospital admission }\end{array}$ \\
\hline Auxiliary variables & $\begin{array}{l}\text { Region (factor), Age category (factor), Week of } \\
\text { symptoms onset (factor), hospitalization in } \\
\text { capitals (factor) and in-hospital mortality (factor) }\end{array}$ \\
\hline
\end{tabular}

Methods used to impute the five covariates

\begin{tabular}{|l|c|c|}
\hline Variable & $\begin{array}{c}\text { Method used for } \\
\text { imputation }\end{array}$ & Command \\
\hline $\begin{array}{l}\text { Sex, ICU admission, Comorbidities, } \\
\text { SARI, Oxygen saturation, Dyspnoea, } \\
\text { Respiratory distress }\end{array}$ & Binary logistic regression & "logit" \\
\hline $\begin{array}{l}\text { Self-reported race, Respiratory } \\
\text { support, Time from symptoms onset } \\
\text { to hospital admission }\end{array}$ & $\begin{array}{c}\text { Multinomial logistic } \\
\text { regression }\end{array}$ & "mlogit" \\
\hline
\end{tabular}


Table A12.8 - Missingness pattern (proportion of missing values in assessed variables) on the population used in the main analysis (RT-qPCR confirmed) ( $n=232,036$ with a defined hospital outcome)

\begin{tabular}{|c|c|c|c|c|c|c|}
\hline & Brazil & North & Northeast & Central-West & Southeast & South \\
\hline Variables, No. (\%) & $(n=232,036)$ & $(n=13,496)$ & $(n=45,238)$ & $(n=17,012)$ & $(n=131,556)$ & $(n=24,734)$ \\
\hline \multicolumn{7}{|l|}{ Covariates } \\
\hline Sex & $41(<0.1 \%)$ & $2(<0.1 \%)$ & $21(<0.1 \%)$ & $1(<0.1 \%)$ & $16(<0.1 \%)$ & $1(<0.1 \%)$ \\
\hline \multicolumn{7}{|l|}{ Comorbidities $^{\text {a }}$} \\
\hline Complete case & 84400 (36\%) & $4212(31 \%)$ & $14214(31 \%)$ & 7794 (46\%) & 46609 (35\%) & $11571(47 \%)$ \\
\hline 1 missing value & $4986(2.1 \%)$ & $283(2.1 \%)$ & $1134(2.5 \%)$ & $447(2.6 \%)$ & 2546 (1.9\%) & $576(2.3 \%)$ \\
\hline 2 missing values & $1025(0.4 \%)$ & $62(0.5 \%)$ & $228(0.5 \%)$ & $96(0.6 \%)$ & $517(0.4 \%)$ & $122(0.5 \%)$ \\
\hline 3 missing values & $328(0.1 \%)$ & $17(0.1 \%)$ & $69(0.2 \%)$ & $27(0.2 \%)$ & $185(0.1 \%)$ & $30(0.1 \%)$ \\
\hline 4 missing values & $210(<0.1 \%)$ & $12(<0.1 \%)$ & $43(<0.1 \%)$ & $14(<0.1 \%)$ & $122(<0.1 \%)$ & $19(<0.1 \%)$ \\
\hline 5 missing values & $703(0.3 \%)$ & $33(0.2 \%)$ & $122(0.3 \%)$ & $31(0.2 \%)$ & $435(0.3 \%)$ & $82(0.3 \%)$ \\
\hline 6 missing values & 3908 (1.7\%) & $140(1.0 \%)$ & $683(1.5 \%)$ & $147(0.9 \%)$ & 2554 (1.9\%) & $384(1.6 \%)$ \\
\hline 7 missing values & 15981 (6.9\%) & $751(5.6 \%)$ & $3212(7.1 \%)$ & $628(3.7 \%)$ & $10208(7.8 \%)$ & $1182(4.8 \%)$ \\
\hline 8 missing values & 29161 (13\%) & $1772(13 \%)$ & $6067(13 \%)$ & $1254(7.4 \%)$ & 18015 (14\%) & $2053(8.3 \%)$ \\
\hline All missing & $91334(39 \%)$ & $6214(46 \%)$ & 19466 (43\%) & $6574(39 \%)$ & $50365(38 \%)$ & 8715 (35\%) \\
\hline \multicolumn{7}{|l|}{$\begin{array}{l}\text { Respiratory } \\
\text { Support }\end{array}$} \\
\hline No & $54314(23 \%)$ & 3047 (23\%) & 8177 (18\%) & 4076 (24\%) & 32756 (25\%) & $6258(25 \%)$ \\
\hline Yes, non-invasive & 96729 (42\%) & $4743(35 \%)$ & 14485 (32\%) & 7561 (44\%) & 58444 (44\%) & $11496(46 \%)$ \\
\hline Yes, invasive & 45205 (19\%) & 3155 (23\%) & $10322(23 \%)$ & 3667 (22\%) & 22648 (17\%) & $5413(22 \%)$ \\
\hline Missing & 35788 (15\%) & 2551 (19\%) & $12254(27 \%)$ & $1708(10 \%)$ & 17708 (13\%) & 1567 (6.3\%) \\
\hline \multicolumn{7}{|l|}{ ICU admission } \\
\hline No & $125806(54 \%)$ & $8187(61 \%)$ & 19665 (43\%) & $9353(55 \%)$ & 73859 (56\%) & $14742(60 \%)$ \\
\hline Yes & 79687 (34\%) & 3786 (28\%) & 14867 (33\%) & $6682(39 \%)$ & 45224 (34\%) & 9128 (37\%) \\
\hline Missing & $26543(11 \%)$ & $1523(11 \%)$ & 10706 (24\%) & 977 (5.7\%) & 12473 (9.5\%) & $864(3.5 \%)$ \\
\hline Hospital outcomes & 254288 & 14712 & 51993 & 18701 & 142963 & 25919 \\
\hline Death & 87515 (34\%) & $6727(46 \%)$ & $21858(42 \%)$ & 5964 (32\%) & $45269(32 \%)$ & 7697 (30\%) \\
\hline Discharge & $144521(57 \%)$ & $6769(46 \%)$ & $23380(45 \%)$ & 11048 (59\%) & $86287(60 \%)$ & 17037 (66\%) \\
\hline Ongoing & 22252 (8.8\%) & $1216(8.3 \%)$ & 6755 (13\%) & $1689(9.0 \%)$ & 11407 (8.0\%) & 1185 (4.6\%) \\
\hline
\end{tabular}

${ }^{a}$ Comorbidities considered: Cardiovascular disease, Diabetes, Kidney disease, Obesity, Neurological disease, Chronic obstructive pulmonary disease, Immunodepression, Haematological disease, and Hepatic disease 

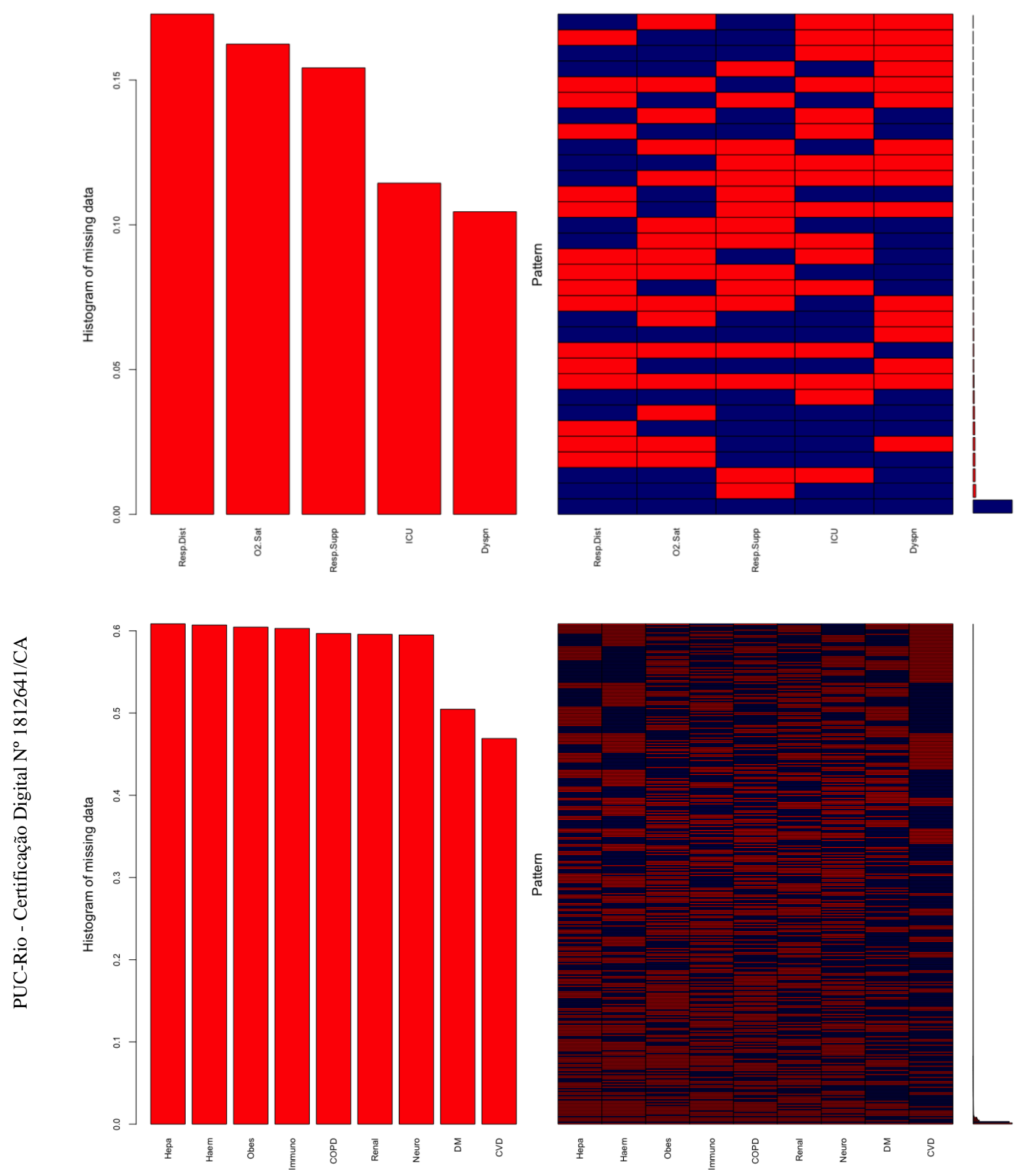

Figure A12.2 - Missingness pattern for ICU, respiratory support, signs/symptoms and comorbidities 
Table A12.9 - Missing vs. not missing comorbidities ( $n=232,036$, sample with defined hospital outcome)

\begin{tabular}{|c|c|c|}
\hline & Missing comorbidities & Not Missing comorbidities \\
\hline Age, median (IQR) & $59(45,72)$ & $65(53,76)$ \\
\hline \multicolumn{3}{|l|}{ Age group, No. (\%) } \\
\hline $20-39$ & $23823(16 \%)$ & $6780(8.0 \%)$ \\
\hline $40-49$ & $24421(17 \%)$ & $9547(11 \%)$ \\
\hline $50-59$ & $27938(19 \%)$ & $15438(18 \%)$ \\
\hline $60-69$ & $28384(19 \%)$ & $19886(24 \%)$ \\
\hline 70-79 & $23477(16 \%)$ & $17957(21 \%)$ \\
\hline $80+$ & $19593(13 \%)$ & $14792(18 \%)$ \\
\hline \multicolumn{3}{|l|}{ Sex, No. (\%) } \\
\hline Female & $62344(42 \%)$ & $38482(46 \%)$ \\
\hline Male & $85266(58 \%)$ & $45903(54 \%)$ \\
\hline Missing & $26(<0.1 \%)$ & $15(<0.1 \%)$ \\
\hline \multicolumn{3}{|l|}{ Self-reported race, No. (\%) * } \\
\hline Black/Brown & $48647(33 \%)$ & $31745(38 \%)$ \\
\hline White & $48315(33 \%)$ & $35108(42 \%)$ \\
\hline Asian & $1601(1.1 \%)$ & $989(1.2 \%)$ \\
\hline Indigenous & $325(0.2 \%)$ & $152(0.2 \%)$ \\
\hline Missing & $48748(33 \%)$ & $16406(19 \%)$ \\
\hline \multicolumn{3}{|l|}{ Respiratory support, No. (\%) } \\
\hline No & 35396 (24\%) & 18918 (22\%) \\
\hline Yes, non-invasive & $57659(39 \%)$ & $39070(46 \%)$ \\
\hline Yes, invasive & $25275(17 \%)$ & $19930(24 \%)$ \\
\hline Missing & $29306(20 \%)$ & $6482(7.7 \%)$ \\
\hline \multicolumn{3}{|l|}{ ICU admission, No. (\%) } \\
\hline No & $78697(53 \%)$ & $47109(56 \%)$ \\
\hline Yes & $46456(31 \%)$ & $33231(39 \%)$ \\
\hline Missing & $22483(15 \%)$ & $4060(4.8 \%)$ \\
\hline \multicolumn{3}{|l|}{ Region, No. (\%) } \\
\hline North & $9284(6.3 \%)$ & $4212(5.0 \%)$ \\
\hline Northeast & $31024(21 \%)$ & $14214(17 \%)$ \\
\hline Central-West & $9218(6.2 \%)$ & $7794(9.2 \%)$ \\
\hline Southeast & $84947(58 \%)$ & $46609(55 \%)$ \\
\hline South & $13163(8.9 \%)$ & $11571(14 \%)$ \\
\hline Hospitalization in capital city, No. (\%) & $85511(58 \%)$ & $40208(48 \%)$ \\
\hline \multicolumn{3}{|l|}{ Outcome, No. (\%) } \\
\hline Death & $51403(35 \%)$ & $36112(43 \%)$ \\
\hline Discharge & $96233(65 \%)$ & $48288(57 \%)$ \\
\hline
\end{tabular}

* Race was collected as self-reported race or skin colour, originally classified as White (Branco), Black (Preto), Brown (Pardo), Asian (Amarelo), and Indigenous (Indígena) 
Table A12.10 - Missing ICU admission versus not missing ICU admission ( $n=232,036$ with a defined hospital outcome)

\begin{tabular}{|c|c|c|}
\hline & Missing ICU & Not missing ICU \\
\hline Age, median (IQR) & $63(49,74)$ & $61(48,73)$ \\
\hline \multicolumn{3}{|l|}{ Age group, No. (\%) } \\
\hline $20-39$ & $3294(12 \%)$ & $27309(13 \%)$ \\
\hline $40-49$ & $3615(14 \%)$ & $30353(15 \%)$ \\
\hline $50-59$ & $4656(18 \%)$ & $38720(19 \%)$ \\
\hline $60-69$ & $5653(21 \%)$ & $42617(21 \%)$ \\
\hline $70-79$ & $5011(19 \%)$ & $36423(18 \%)$ \\
\hline $80+$ & $4314(16 \%)$ & $30071(15 \%)$ \\
\hline \multicolumn{3}{|l|}{ Sex, No. (\%) } \\
\hline Female & $11911(45 \%)$ & $88915(43 \%)$ \\
\hline Male & $14616(55 \%)$ & $116553(57 \%)$ \\
\hline Missing & $16(<0.1 \%)$ & $25(<0.1 \%)$ \\
\hline \multicolumn{3}{|l|}{ Number of comorbidities, No. (\%) } \\
\hline No comorbidities & 794 (3.0\%) & $14183(6.9 \%)$ \\
\hline $1-2$ & $10002(38 \%)$ & $102934(50 \%)$ \\
\hline$>=3$ & $721(2.7 \%)$ & $12068(5.9 \%)$ \\
\hline Missing & $15026(57 \%)$ & $76308(37 \%)$ \\
\hline \multicolumn{3}{|l|}{ Self-reported race, No. $(\%)^{*}$} \\
\hline Black/Brown & $9261(35 \%)$ & $71131(35 \%)$ \\
\hline White & $6008(23 \%)$ & $77415(38 \%)$ \\
\hline Asian & $318(1.2 \%)$ & $2272(1.1 \%)$ \\
\hline Indigenous & $58(0.2 \%)$ & $419(0.2 \%)$ \\
\hline Missing & $10898(41 \%)$ & $54256(26 \%)$ \\
\hline \multicolumn{3}{|l|}{ Respiratory support, No. (\%) } \\
\hline No & $1395(5.3 \%)$ & $52919(26 \%)$ \\
\hline Yes, non-invasive & $4913(19 \%)$ & $91816(45 \%)$ \\
\hline Yes, invasive & $1150(4.3 \%)$ & $44055(21 \%)$ \\
\hline Missing & $19085(72 \%)$ & $16703(8.1 \%)$ \\
\hline \multicolumn{3}{|l|}{ Region, No. (\%) } \\
\hline North & $1523(5.7 \%)$ & $11973(5.8 \%)$ \\
\hline Northeast & $10706(40 \%)$ & $34532(17 \%)$ \\
\hline Central-West & $977(3.7 \%)$ & $16035(7.8 \%)$ \\
\hline Southeast & $12473(47 \%)$ & $119083(58 \%)$ \\
\hline South & $864(3.3 \%)$ & $23870(12 \%)$ \\
\hline Hospitalization in capital city, No. (\%) & $16650(63 \%)$ & $109069(53 \%)$ \\
\hline \multicolumn{3}{|l|}{ Outcome, No. (\%) } \\
\hline Death & $11152(42 \%)$ & $76363(37 \%)$ \\
\hline Discharge & $15391(58 \%)$ & $129130(63 \%)$ \\
\hline
\end{tabular}

${ }^{*}$ Race was collected as self-reported race or skin colour, originally classified as White (Branco), Black (Preto), Brown (Pardo), Asian (Amarelo), and Indigenous (Indígena) 
Table A12.11 - Missing Respiratory support versus not missing Respiratory support (sample $\mathrm{n}=232,036$ with a defined hospital outcome)

\begin{tabular}{|c|c|c|}
\hline & $\begin{array}{c}\text { Missing } \\
\text { respiratory support }\end{array}$ & $\begin{array}{l}\text { Not missing } \\
\text { respiratory support }\end{array}$ \\
\hline Age, median (IQR) & $62(48,74)$ & $61(48,73)$ \\
\hline \multicolumn{3}{|l|}{ Age group, No. (\%) } \\
\hline $20-39$ & $4732(13 \%)$ & $25871(13 \%)$ \\
\hline $40-49$ & $5202(15 \%)$ & $28766(15 \%)$ \\
\hline $50-59$ & $6372(18 \%)$ & $37004(19 \%)$ \\
\hline $60-69$ & $7459(21 \%)$ & $40811(21 \%)$ \\
\hline $70-79$ & $6521(18 \%)$ & $34913(18 \%)$ \\
\hline $80+$ & $5502(15 \%)$ & $28883(15 \%)$ \\
\hline \multicolumn{3}{|l|}{ Sex, No. (\%) } \\
\hline Female & $15798(44 \%)$ & $85028(43 \%)$ \\
\hline Male & $19972(56 \%)$ & $111197(57 \%)$ \\
\hline Missing & $18(<0.1 \%)$ & $23(<0.1 \%)$ \\
\hline \multicolumn{3}{|l|}{ Number of comorbidities, No. (\%) } \\
\hline No comorbidities & $1417(4.0 \%)$ & $13560(6.9 \%)$ \\
\hline $1-2$ & $13447(38 \%)$ & $99489(51 \%)$ \\
\hline$>=3$ & $1015(2.8 \%)$ & $11774(6.0 \%)$ \\
\hline Missing & $19909(56 \%)$ & $71425(36 \%)$ \\
\hline \multicolumn{3}{|l|}{ Self-reported race, No. $(\%)^{*}$} \\
\hline Black/Brown & $12278(34 \%)$ & $68114(35 \%)$ \\
\hline White & $8562(24 \%)$ & $74861(38 \%)$ \\
\hline Asian & $460(1.3 \%)$ & $2130(1.1 \%)$ \\
\hline Indigenous & $87(0.2 \%)$ & $390(0.2 \%)$ \\
\hline Missing & $14401(40 \%)$ & $50753(26 \%)$ \\
\hline \multicolumn{3}{|l|}{ ICU admission, No. (\%) } \\
\hline No & $11000(31 \%)$ & $114806(59 \%)$ \\
\hline Yes & $5703(16 \%)$ & $73984(38 \%)$ \\
\hline Missing & $19085(53 \%)$ & $7458(3.8 \%)$ \\
\hline \multicolumn{3}{|l|}{ Region, No. (\%) } \\
\hline North & $2551(7.1 \%)$ & $10945(5.6 \%)$ \\
\hline Northeast & $12254(34 \%)$ & $32984(17 \%)$ \\
\hline Central-West & $1708(4.8 \%)$ & $15304(7.8 \%)$ \\
\hline Southeast & $17708(49 \%)$ & $113848(58 \%)$ \\
\hline South & $1567(4.4 \%)$ & $23167(12 \%)$ \\
\hline Hospitalization in capital city, No. (\%) & $20950(59 \%)$ & $104769(53 \%)$ \\
\hline \multicolumn{3}{|l|}{ Outcome, No. (\%) } \\
\hline Death & $14527(41 \%)$ & $72988(37 \%)$ \\
\hline Discharge & $21261(59 \%)$ & $123260(63 \%)$ \\
\hline
\end{tabular}


Table A12.12 - Comparison between complete-case and imputed values (sample $n=232,036$ with a defined hospital outcome)

\begin{tabular}{|c|c|c|}
\hline Imputed variables & Original (complete cases) & Imputed values \\
\hline \multicolumn{3}{|l|}{ Sex } \\
\hline Female & $100,826 / 231,995(43 \%)$ & $43.5 \%$ \\
\hline Male & $131,169 / 231,995(57 \%)$ & $56.5 \%$ \\
\hline \multicolumn{3}{|l|}{ Self-reported race * } \\
\hline White & $83,423 / 166,882(50 \%)$ & $48.1 \%$ \\
\hline Black/Brown & $80,392 / 166,882(48 \%)$ & $50.0 \%$ \\
\hline Asian & $2,590 / 166,882(1.6 \%)$ & $1.6 \%$ \\
\hline Indigenous & $477 / 166,882(0.3 \%)$ & $0.3 \%$ \\
\hline \multicolumn{3}{|l|}{ Sign/Symptoms } \\
\hline Oxygen Saturation $<95 \%$ & $135,620 / 194,351(70 \%)$ & $70.8 \%$ \\
\hline Dyspnoea & $165,977 / 207,780(80 \%)$ & $80.4 \%$ \\
\hline Respiratory distress & $132,188 / 191,943(69 \%)$ & $70.5 \%$ \\
\hline SARI & $117,832 / 193,494(61 \%)$ & $62.3 \%$ \\
\hline \multicolumn{3}{|l|}{ Comorbidities } \\
\hline Cardiovascular disease & $81,156 / 123,187(66 \%)$ & $64.2 \%$ \\
\hline Diabetes & $61,537 / 114,921(54 \%)$ & $54.1 \%$ \\
\hline Obesity & $11,617 / 91,744(13 \%)$ & $18.0 \%$ \\
\hline Kidney disease & $10,676 / 93,806(11 \%)$ & $16.7 \%$ \\
\hline COPD & $9,290 / 93,565(10 \%)$ & $14.0 \%$ \\
\hline Neurological disease & $9,654 / 93,969(10 \%)$ & $13.4 \%$ \\
\hline Immunodepression & $6,849 / 92,142(7 \%)$ & $11.7 \%$ \\
\hline Hepatic disease & $2,240 / 90,845(3 \%)$ & $7.1 \%$ \\
\hline Haematological disease & $1,963 / 91,161(2 \%)$ & $6.9 \%$ \\
\hline \multicolumn{3}{|l|}{ Number of comorbidities } \\
\hline 0 & $13,836 / 84,400(16 \%)$ & $13.3 \%$ \\
\hline $1-2$ & $62,766 / 84,400(74 \%)$ & $60.6 \%$ \\
\hline$\geq 3$ & $7,798 / 84,400(9 \%)$ & $26.1 \%$ \\
\hline \multicolumn{3}{|l|}{$\begin{array}{l}\text { Time from onset of symptoms to } \\
\text { hospital admission }\end{array}$} \\
\hline$<=3$ days & $74,728 / 228,447$ (33\%) & $32.7 \%$ \\
\hline$<=6$ days & $56,914 / 228,447$ (25\%) & $24.9 \%$ \\
\hline$<=9$ days & $51,416 / 228,447(23 \%)$ & $22.5 \%$ \\
\hline$<=12$ days & $25,007 / 228,447(11 \%)$ & $10.9 \%$ \\
\hline$<=15$ days & $11,684 / 228,447(5 \%)$ & $5.1 \%$ \\
\hline$>15$ days & $8,698 / 228,447(4 \%)$ & $3.8 \%$ \\
\hline \multicolumn{3}{|l|}{ Respiratory Support } \\
\hline None & $54,314 / 196,248(28 \%)$ & $27.7 \%$ \\
\hline Yes, non-invasive & $96,729 / 196,248(49 \%)$ & $49.1 \%$ \\
\hline Yes, invasive & $45,205 / 196,248(23 \%)$ & $23.2 \%$ \\
\hline ICU admission & $79,687 / 205,493(39 \%)$ & $38.9 \%$ \\
\hline
\end{tabular}


12.3.3

Appendix A4.3 - Supplementary analyses of COVID-19 hospital admissions, use of resources and outcomes
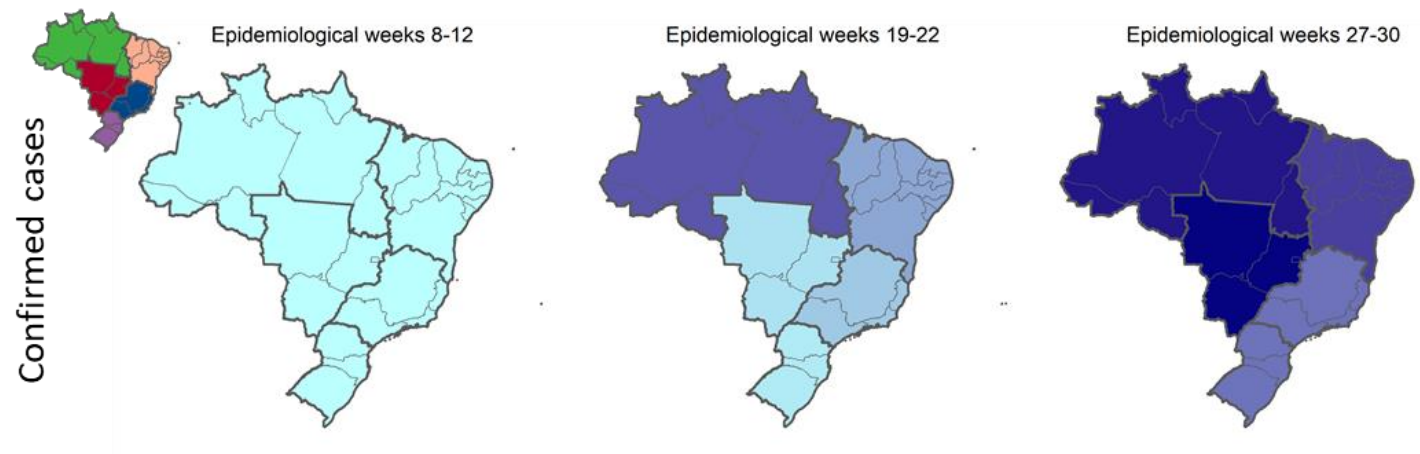

per 100,000
population

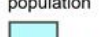

อง
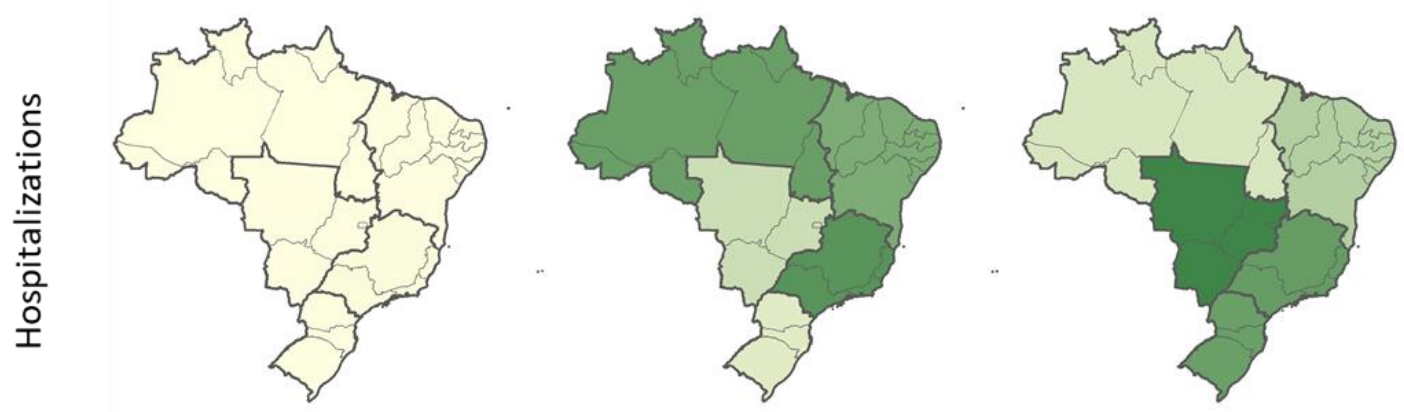

per 100,000 population
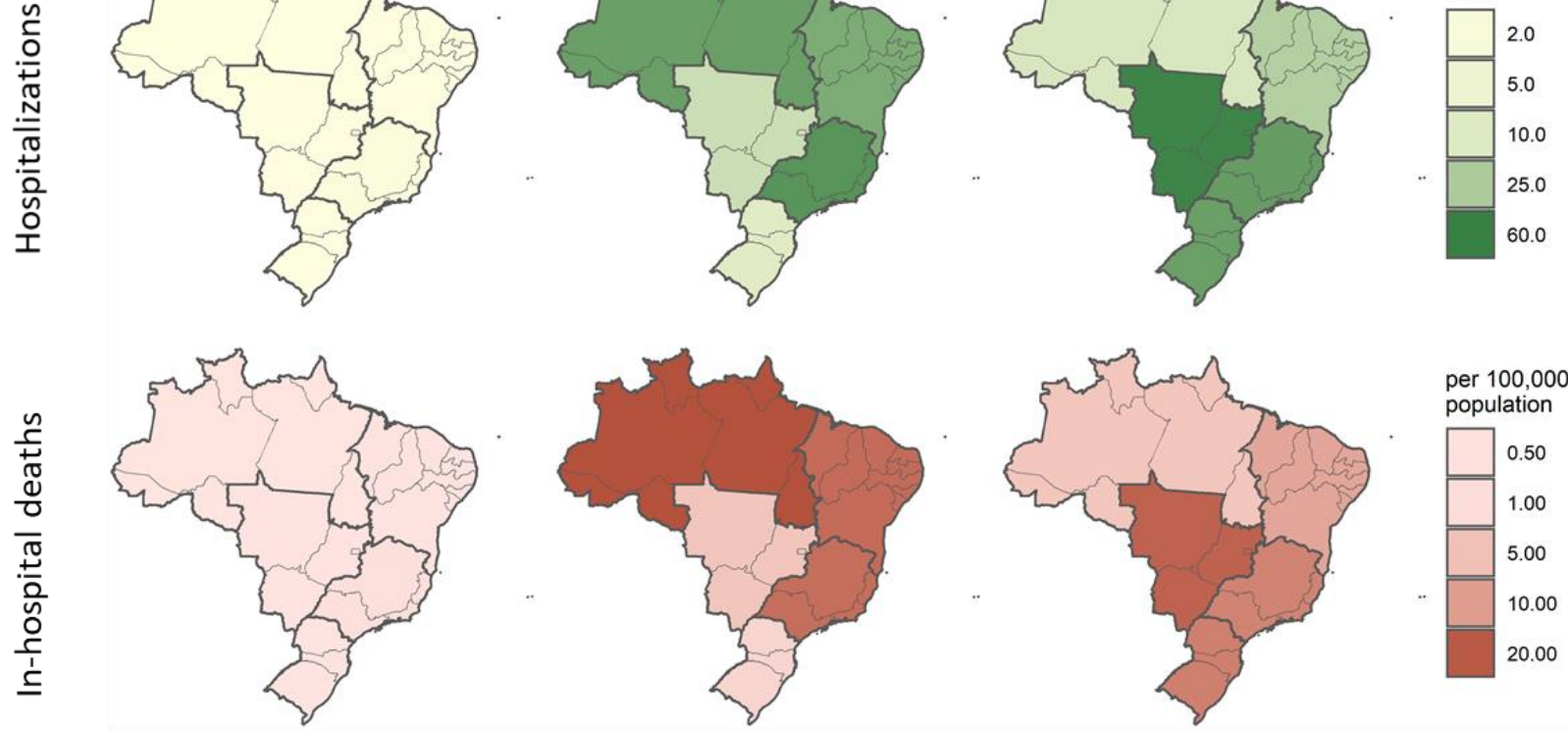

per 100,000 population

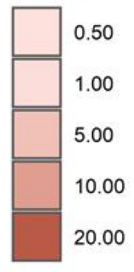

Figure A12.3 - Epidemic evolution showed during three-time frames in Brazil with rates per 100,000 population 
Table A12.13 - Number of COVID-19 cases, hospitalisations, and in-hospital deaths, absolute and age and sex-adjusted rates per 100,000 population for each time frame of the pandemic and region of Brazil

\section{Epidemiological Weeks}

\begin{tabular}{|c|c|c|c|c|}
\hline Region & 8 to 12 & 19 to 22 & 27 to 30 & Overall \\
\hline \multicolumn{5}{|l|}{ Brazil } \\
\hline Population (Total) & & & & $211,755,692$ \\
\hline Confirmed Cases & 1,060 & 402,336 & $1,066,763$ & $3,278,839$ \\
\hline Rate per 100,000 population & 0.5 & 190.0 & 503.8 & $1,548.4$ \\
\hline Population (Adults) & & & & $151,778,729$ \\
\hline Hospitalisation & 1,243 & 58,292 & 57,615 & 254,288 \\
\hline Rate per 100,000 population & 0.8 & 38.4 & 38.0 & 167.5 \\
\hline In-hospital Deaths & 440 & 21,615 & 18,501 & 87,515 \\
\hline Rate per 100,000 population & 0.3 & 14.2 & 12.2 & 57.7 \\
\hline
\end{tabular}

* Brazilian 2020 projected population as reference.

\begin{tabular}{|l|c|c|c|c|c|c|}
\hline \multicolumn{1}{|c|}{ Region } & Hospitalisations & $\begin{array}{c}\text { Crude rate } \\
\text { per 100,000 } \\
\text { population }\end{array}$ & $\begin{array}{c}\text { Age-and-sex } \\
\text { adj. rate per } \\
\mathbf{1 0 0 , 0 0 0} \\
\text { population* }\end{array}$ & $\begin{array}{c}\text { In-hospital } \\
\text { Deaths }\end{array}$ & $\begin{array}{c}\text { Crude rate } \\
\text { per 100,000 } \\
\text { population }\end{array}$ & $\begin{array}{c}\text { Age-and-sex } \\
\text { adj. rate per } \\
\mathbf{1 0 0 , 0 0 0} \\
\text { population* }\end{array}$ \\
\hline North & 14,712 & 122.1 & 153.5 & 6,727 & 55.8 & 76.3 \\
\hline Northeast & 51,993 & 130.4 & 137.1 & 21,858 & 54.8 & 58.3 \\
\hline $\begin{array}{l}\text { Central- } \\
\text { West }\end{array}$ & 18,701 & 160.1 & 172.9 & 5,964 & 51.1 & 58.5 \\
\hline Southeast & 142,963 & 217.3 & 207.8 & 45,269 & 68.8 & 64.1 \\
\hline South & 25,919 & 115.9 & 109.3 & 7,697 & 34.4 & 31.3 \\
\hline
\end{tabular}


Table A12.14 - Symptoms of hospitalised COVID-19 patients in Brazil and regions (sample $\mathrm{n}=254,288$ )

\begin{tabular}{|c|c|c|c|c|c|c|}
\hline Symptoms & Brazil & North & $\begin{array}{l}\text { Northea } \\
\text { st }\end{array}$ & $\begin{array}{c}\text { Central- } \\
\text { West }\end{array}$ & $\begin{array}{c}\text { Southea } \\
\text { st }\end{array}$ & South \\
\hline $\begin{array}{l}\text { Cough, No. } \\
(\%)[n= \\
229323(90 \%)]\end{array}$ & $\begin{array}{c}188423 / \\
229323 \\
(82 \%)\end{array}$ & $\begin{array}{l}11947 / \\
13615 \\
(88 \%)\end{array}$ & $\begin{array}{c}37703 / \\
44796 \\
(84 \%)\end{array}$ & $\begin{array}{c}13747 / \\
17705 \\
(78 \%)\end{array}$ & $\begin{array}{c}105914 / \\
128913 \\
(82 \%)\end{array}$ & $\begin{array}{l}19112 / \\
24294 \\
(79 \%)\end{array}$ \\
\hline $\begin{array}{l}\text { Fever, No. (\%) } \\
{[n=226013} \\
(89 \%)]\end{array}$ & $\begin{array}{c}171396 / \\
226013 \\
(76 \%)\end{array}$ & $\begin{array}{l}11975 / \\
13668 \\
(88 \%)\end{array}$ & $\begin{array}{c}35539 / \\
43823 \\
(81 \%)\end{array}$ & $\begin{array}{c}12602 / \\
17507 \\
(72 \%)\end{array}$ & $\begin{array}{c}94565 / \\
127028 \\
(74 \%)\end{array}$ & $\begin{array}{l}16715 / \\
23987 \\
(70 \%)\end{array}$ \\
\hline $\begin{array}{l}\text { Dyspnoea, No. } \\
(\%)[n= \\
226724(89 \%)]\end{array}$ & $\begin{array}{c}180818 / \\
226724 \\
(80 \%)\end{array}$ & $\begin{array}{l}11379 / \\
13532 \\
(84 \%)\end{array}$ & $\begin{array}{c}36883 / \\
44413 \\
(83 \%)\end{array}$ & $\begin{array}{l}13709 / \\
17694 \\
(77 \%)\end{array}$ & $\begin{array}{c}99548 / \\
126589 \\
(79 \%)\end{array}$ & $\begin{array}{l}19299 / \\
24496 \\
(79 \%)\end{array}$ \\
\hline $\begin{array}{l}\text { Oxygen } \\
\text { saturation < } \\
95 \% \text {, No. (\%) } \\
{[n=212016} \\
(83 \%)]\end{array}$ & $\begin{array}{c}147596 / \\
212016 \\
(70 \%)\end{array}$ & $\begin{array}{l}7955 / \\
11901 \\
(67 \%)\end{array}$ & $\begin{array}{l}27410 / \\
39688 \\
(69 \%)\end{array}$ & $\begin{array}{l}10913 / \\
17097 \\
(64 \%)\end{array}$ & $\begin{array}{c}85739 / \\
120027 \\
(71 \%)\end{array}$ & $\begin{array}{l}15579 / \\
23303 \\
(67 \%)\end{array}$ \\
\hline $\begin{array}{l}\text { Respiratory } \\
\text { distress, No. } \\
(\%)[n= \\
209145(82 \%)]\end{array}$ & $\begin{array}{c}143977 / \\
209145 \\
(69 \%)\end{array}$ & $\begin{array}{l}9802 / \\
12538 \\
(78 \%)\end{array}$ & $\begin{array}{c}26737 / \\
38207 \\
(70 \%)\end{array}$ & $\begin{array}{c}11286 / \\
17083 \\
(66 \%)\end{array}$ & $\begin{array}{c}80530 / \\
118114 \\
(68 \%)\end{array}$ & $\begin{array}{l}15622 / \\
23203 \\
(67 \%)\end{array}$ \\
\hline $\begin{array}{l}\text { Sore throat, } \\
\text { No. }(\%)[n= \\
185936(73 \%)]\end{array}$ & $\begin{array}{c}46239 / \\
185936 \\
(25 \%)\end{array}$ & $\begin{array}{l}5193 / \\
11638 \\
(45 \%)\end{array}$ & $\begin{array}{l}8130 / \\
31754 \\
(26 \%)\end{array}$ & $\begin{array}{l}3052 / \\
16059 \\
(19 \%)\end{array}$ & $\begin{array}{c}24619 / \\
104815 \\
(23 \%)\end{array}$ & $\begin{array}{l}5245 / \\
21670 \\
(24 \%)\end{array}$ \\
\hline $\begin{array}{l}\text { Diarrhoea, No. } \\
(\%)[n= \\
182938(72 \%)]\end{array}$ & $\begin{array}{c}34515 / \\
182938 \\
(19 \%)\end{array}$ & $\begin{array}{l}2338 / \\
11157 \\
(21 \%)\end{array}$ & $\begin{array}{l}5855 / \\
31356 \\
(19 \%)\end{array}$ & $\begin{array}{l}2556 / \\
16170 \\
(16 \%)\end{array}$ & $\begin{array}{c}19485 / \\
102533 \\
(19 \%)\end{array}$ & $\begin{array}{l}4281 / \\
21722 \\
(20 \%)\end{array}$ \\
\hline $\begin{array}{l}\text { Vomit, No. (\%) } \\
{[n=178603} \\
(70 \%)]\end{array}$ & $\begin{array}{c}19802 / \\
178603 \\
(11 \%)\end{array}$ & $\begin{array}{l}1199 / \\
10859 \\
(11 \%)\end{array}$ & $\begin{array}{l}3321 / \\
30394 \\
(11 \%)\end{array}$ & $\begin{array}{l}1518 / \\
15954 \\
(9.5 \%)\end{array}$ & $\begin{array}{c}11240 / \\
100040 \\
(11 \%)\end{array}$ & $\begin{array}{l}2524 / \\
21356 \\
(12 \%)\end{array}$ \\
\hline $\begin{array}{l}\text { Other } \\
\text { symptoms, No. } \\
(\%)[n= \\
182647(72 \%)]\end{array}$ & $\begin{array}{c}87316 / \\
182647 \\
(48 \%)\end{array}$ & $\begin{array}{l}4305 / \\
10661 \\
(40 \%)\end{array}$ & $\begin{array}{c}17327 / \\
33223 \\
(52 \%)\end{array}$ & $\begin{array}{l}7796 / \\
15758 \\
(49 \%)\end{array}$ & $\begin{array}{c}47292 / \\
101788 \\
(46 \%)\end{array}$ & $\begin{array}{l}10596 / \\
21217 \\
(50 \%)\end{array}$ \\
\hline
\end{tabular}


Table A12.15 -- Chronic comorbidities description of hospitalised COVID-19 patients in Brazil (sample $n=254,288$ )

\begin{tabular}{|c|c|c|c|c|c|c|}
\hline Comorbidities & Brazil & North & Northeast & Central-West & Southeast & South \\
\hline \multicolumn{7}{|c|}{ Cardiovascular disease, №. (\%) } \\
\hline No & $\begin{array}{c}45248 / 254288 \\
(18 \%)\end{array}$ & $\begin{array}{c}2563 / 14712 \\
(17 \%)\end{array}$ & $\begin{array}{c}8645 / 51993 \\
(17 \%)\end{array}$ & $\begin{array}{c}4549 \text { / } 18701 \\
(24 \%)\end{array}$ & $\begin{array}{c}23465 / 142963 \\
(16 \%)\end{array}$ & $\begin{array}{c}6026 / 25919 \\
(23 \%)\end{array}$ \\
\hline Yes & $\begin{array}{c}88279 / 254288 \\
(35 \%)\end{array}$ & $\begin{array}{c}4049 / 14712 \\
(28 \%)\end{array}$ & $\begin{array}{c}16310 / 51993 \\
(31 \%)\end{array}$ & $\begin{array}{c}5811 / 18701 \\
(31 \%)\end{array}$ & $\begin{array}{c}53015 / 142963 \\
(37 \%)\end{array}$ & $\begin{array}{c}9094 / 25919 \\
(35 \%)\end{array}$ \\
\hline Missing & $\begin{array}{c}120761 / 254288 \\
(47 \%)\end{array}$ & $\begin{array}{c}8100 / 14712 \\
(55 \%)\end{array}$ & $\begin{array}{c}27038 / 51993 \\
(52 \%)\end{array}$ & $\begin{array}{c}8341 / 18701 \\
(45 \%)\end{array}$ & $\begin{array}{c}66483 / 142963 \\
(47 \%)\end{array}$ & $\begin{array}{c}10799 / 25919 \\
(42 \%)\end{array}$ \\
\hline \multicolumn{7}{|c|}{ Diabetes, No. (\%) } \\
\hline 1 & $\begin{array}{c}57461 / 254288 \\
(23 \%)\end{array}$ & $\begin{array}{c}2748 / 14712 \\
(19 \%)\end{array}$ & $\begin{array}{c}9901 / 51993 \\
(19 \%)\end{array}$ & $\begin{array}{c}5382 / 18701 \\
(29 \%)\end{array}$ & $\begin{array}{c}31378 / 142963 \\
(22 \%)\end{array}$ & $\begin{array}{c}8052 \text { / } 25919 \\
(31 \%)\end{array}$ \\
\hline Yes & $\begin{array}{c}66871 / 254288 \\
(26 \%)\end{array}$ & $\begin{array}{c}3605 / 14712 \\
(25 \%)\end{array}$ & $\begin{array}{c}14145 / 51993 \\
(27 \%)\end{array}$ & $\begin{array}{c}4896 / 18701 \\
(26 \%)\end{array}$ & $\begin{array}{c}37742 / 142963 \\
(26 \%)\end{array}$ & $\begin{array}{c}6483 / 25919 \\
(25 \%)\end{array}$ \\
\hline Missing & $\begin{array}{c}129956 / 254288 \\
(51 \%)\end{array}$ & $\begin{array}{c}8359 / 14712 \\
(57 \%)\end{array}$ & $\begin{array}{c}27947 / 51993 \\
(54 \%)\end{array}$ & $\begin{array}{c}8423 / 18701 \\
(45 \%)\end{array}$ & $\begin{array}{c}73843 / 142963 \\
(52 \%)\end{array}$ & $\begin{array}{c}11384 / 25919 \\
(44 \%)\end{array}$ \\
\hline \multicolumn{7}{|c|}{ Kidney disease, No. (\%) } \\
\hline No & $\begin{array}{c}89542 \text { / } 254288 \\
(35 \%)\end{array}$ & $\begin{array}{c}4449 \text { / } 14712 \\
(30 \%)\end{array}$ & $\begin{array}{c}16024 / 51993 \\
(31 \%)\end{array}$ & $\begin{array}{c}8464 \text { / } 18701 \\
(45 \%)\end{array}$ & $\begin{array}{c}48837 / 142963 \\
(34 \%)\end{array}$ & $\begin{array}{c}11768 / 25919 \\
(45 \%)\end{array}$ \\
\hline Yes & $\begin{array}{c}11467 / 254288 \\
(4.5 \%)\end{array}$ & $\begin{array}{c}634 / 14712 \\
(4.3 \%)\end{array}$ & $\begin{array}{c}2378 / 51993 \\
(4.6 \%)\end{array}$ & $\begin{array}{c}784 \text { / } 18701 \\
(4.2 \%)\end{array}$ & $\begin{array}{c}6383 / 142963 \\
(4.5 \%)\end{array}$ & $\begin{array}{c}1288 / 25919 \\
(5.0 \%)\end{array}$ \\
\hline Missing & $\begin{array}{c}153279 \text { / } 254288 \\
(60 \%)\end{array}$ & $\begin{array}{c}9629 / 14712 \\
(65 \%)\end{array}$ & $\begin{array}{c}33591 / 51993 \\
(65 \%)\end{array}$ & $\begin{array}{c}9453 / 18701 \\
(51 \%)\end{array}$ & $\begin{array}{c}87743 / 142963 \\
(61 \%)\end{array}$ & $\begin{array}{c}12863 / 25919 \\
(50 \%)\end{array}$ \\
\hline \multicolumn{7}{|l|}{ Obesity, No. (\%) } \\
\hline 年 & $\begin{array}{c}86270 / 254288 \\
(34 \%)\end{array}$ & $\begin{array}{c}4525 / 14712 \\
(31 \%)\end{array}$ & $\begin{array}{c}15850 / 51993 \\
(30 \%)\end{array}$ & $\begin{array}{c}8093 / 18701 \\
(43 \%)\end{array}$ & $\begin{array}{c}46730 / 142963 \\
(33 \%)\end{array}$ & $\begin{array}{c}11072 \text { / } 25919 \\
(43 \%)\end{array}$ \\
\hline es & $\begin{array}{c}12556 / 254288 \\
(4.9 \%)\end{array}$ & $\begin{array}{c}355 / 14712 \\
(2.4 \%)\end{array}$ & $\begin{array}{c}1714 \text { / } 51993 \\
(3.3 \%)\end{array}$ & $\begin{array}{c}991 / 18701 \\
(5.3 \%)\end{array}$ & $\begin{array}{c}7520 / 142963 \\
(5.3 \%)\end{array}$ & $\begin{array}{c}1976 / 25919 \\
(7.6 \%)\end{array}$ \\
\hline 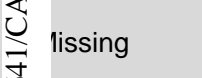 & $\begin{array}{c}155462 / 254288 \\
(61 \%)\end{array}$ & $\begin{array}{c}9832 / 14712 \\
(67 \%)\end{array}$ & $\begin{array}{c}34429 / 51993 \\
(66 \%)\end{array}$ & $\begin{array}{c}9617 / 18701 \\
(51 \%)\end{array}$ & $\begin{array}{c}88713 / 142963 \\
(62 \%)\end{array}$ & $\begin{array}{c}12871 / 25919 \\
(50 \%)\end{array}$ \\
\hline \multicolumn{7}{|c|}{ త Arological disease, No. (\%) } \\
\hline 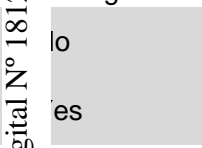 & $\begin{array}{c}90869 / 254288 \\
(36 \%) \\
10299 / 254288 \\
(4.1 \%)\end{array}$ & $\begin{array}{c}4707 / 14712 \\
(32 \%) \\
292 / 14712 \\
(2.0 \%)\end{array}$ & $\begin{array}{c}16642 / 51993 \\
(32 \%) \\
1592 / 51993 \\
(3.1 \%)\end{array}$ & $\begin{array}{c}8602 / 18701 \\
(46 \%) \\
610 / 18701 \\
(3.3 \%)\end{array}$ & $\begin{array}{c}49275 / 142963 \\
(34 \%) \\
6308 / 142963 \\
(4.4 \%)\end{array}$ & $\begin{array}{c}11643 / 25919 \\
(45 \%) \\
1497 / 25919 \\
(5.8 \%)\end{array}$ \\
\hline 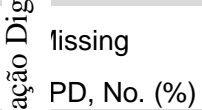 & $\begin{array}{c}153120 / 254288 \\
(60 \%)\end{array}$ & $\begin{array}{c}9713 / 14712 \\
(66 \%)\end{array}$ & $\begin{array}{c}33759 / 51993 \\
(65 \%)\end{array}$ & $\begin{array}{c}9489 / 18701 \\
(51 \%)\end{array}$ & $\begin{array}{c}87380 / 142963 \\
(61 \%)\end{array}$ & $\begin{array}{c}12779 \text { / } 25919 \\
(49 \%)\end{array}$ \\
\hline$\overbrace{0}^{\mathscr{E}} 10$ & $\begin{array}{c}90816 / 254288 \\
(36 \%)\end{array}$ & $\begin{array}{c}4631 / 14712 \\
(31 \%)\end{array}$ & $\begin{array}{c}16755 / 51993 \\
(32 \%)\end{array}$ & $\begin{array}{c}8487 / 18701 \\
(45 \%)\end{array}$ & $\begin{array}{c}49375 / 142963 \\
(35 \%)\end{array}$ & $\begin{array}{c}11568 / 25919 \\
(45 \%)\end{array}$ \\
\hline$\bigcup_{0}^{\infty}$ es & $\begin{array}{c}9914 / 254288 \\
(3.9 \%)\end{array}$ & $\begin{array}{c}388 / 14712 \\
(2.6 \%)\end{array}$ & $\begin{array}{c}1370 / 51993 \\
(2.6 \%)\end{array}$ & $\begin{array}{c}792 / 18701 \\
(4.2 \%)\end{array}$ & $\begin{array}{c}5768 / 142963 \\
(4.0 \%)\end{array}$ & $\begin{array}{c}1596 / 25919 \\
(6.2 \%)\end{array}$ \\
\hline لئ & $\begin{array}{c}153558 / 254288 \\
(60 \%)\end{array}$ & $\begin{array}{c}9693 / 14712 \\
(66 \%)\end{array}$ & $\begin{array}{c}33868 / 51993 \\
(65 \%)\end{array}$ & $\begin{array}{c}9422 / 18701 \\
(50 \%)\end{array}$ & $\begin{array}{c}87820 / 142963 \\
(61 \%)\end{array}$ & $\begin{array}{c}12755 / 25919 \\
(49 \%)\end{array}$ \\
\hline \multicolumn{7}{|c|}{ inodepression, No. (\%) } \\
\hline No & $\begin{array}{c}91884 \text { / } 254288 \\
(36 \%)\end{array}$ & $\begin{array}{c}4584 \text { / } 14712 \\
(31 \%)\end{array}$ & $\begin{array}{c}16586 / 51993 \\
(32 \%)\end{array}$ & $\begin{array}{c}8715 / 18701 \\
(47 \%)\end{array}$ & $\begin{array}{c}50070 / 142963 \\
(35 \%)\end{array}$ & $\begin{array}{c}11929 / 25919 \\
(46 \%)\end{array}$ \\
\hline Yes & $\begin{array}{c}7314 \text { / } 254288 \\
(2.9 \%)\end{array}$ & $\begin{array}{c}417 / 14712 \\
(2.8 \%)\end{array}$ & $\begin{array}{c}1315 \text { / } 51993 \\
(2.5 \%)\end{array}$ & $\begin{array}{c}429 / 18701 \\
(2.3 \%)\end{array}$ & $\begin{array}{c}4123 / 142963 \\
(2.9 \%)\end{array}$ & $\begin{array}{c}1030 / 25919 \\
(4.0 \%)\end{array}$ \\
\hline Missing & $\begin{array}{c}155090 / 254288 \\
(61 \%)\end{array}$ & $\begin{array}{c}9711 / 14712 \\
(66 \%)\end{array}$ & $\begin{array}{c}34092 / 51993 \\
(66 \%)\end{array}$ & $\begin{array}{c}9557 / 18701 \\
(51 \%)\end{array}$ & $\begin{array}{c}88770 / 142963 \\
(62 \%)\end{array}$ & $\begin{array}{c}12960 / 25919 \\
(50 \%)\end{array}$ \\
\hline \multicolumn{7}{|l|}{ Asthma, No. (\%) } \\
\hline No & $\begin{array}{c}92690 / 254288 \\
(36 \%)\end{array}$ & $\begin{array}{c}4684 / 14712 \\
(32 \%)\end{array}$ & $\begin{array}{c}17041 / 51993 \\
(33 \%)\end{array}$ & $\begin{array}{c}8692 \text { / } 18701 \\
(46 \%)\end{array}$ & $\begin{array}{c}50273 / 142963 \\
(35 \%)\end{array}$ & $\begin{array}{c}12000 / 25919 \\
(46 \%)\end{array}$ \\
\hline Yes & $\begin{array}{c}6858 / 254288 \\
(2.7 \%)\end{array}$ & $\begin{array}{c}300 / 14712 \\
(2.0 \%)\end{array}$ & $\begin{array}{c}988 / 51993 \\
(1.9 \%)\end{array}$ & $\begin{array}{c}516 / 18701 \\
(2.8 \%)\end{array}$ & $\begin{array}{c}4032 \text { / } 142963 \\
(2.8 \%)\end{array}$ & $\begin{array}{c}1022 / 25919 \\
(3.9 \%)\end{array}$ \\
\hline Missing & $\begin{array}{c}154740 / 254288 \\
(61 \%)\end{array}$ & $\begin{array}{c}9728 / 14712 \\
(66 \%)\end{array}$ & $\begin{array}{c}33964 / 51993 \\
(65 \%)\end{array}$ & $\begin{array}{c}9493 / 18701 \\
(51 \%)\end{array}$ & $\begin{array}{c}88658 / 142963 \\
(62 \%)\end{array}$ & $\begin{array}{c}12897 / 25919 \\
(50 \%)\end{array}$ \\
\hline \multicolumn{7}{|c|}{ Haematological disease, No. (\%) } \\
\hline No & $\begin{array}{c}96004 \text { / } 254288 \\
(38 \%)\end{array}$ & $\begin{array}{c}4831 / 14712 \\
(33 \%)\end{array}$ & $\begin{array}{c}17390 / 51993 \\
(33 \%)\end{array}$ & $\begin{array}{c}9013 / 18701 \\
(48 \%)\end{array}$ & $\begin{array}{c}52164 / 142963 \\
(36 \%)\end{array}$ & $\begin{array}{c}12606 / 25919 \\
(49 \%)\end{array}$ \\
\hline Yes & $\begin{array}{c}2130 / 254288 \\
(0.8 \%)\end{array}$ & $\begin{array}{c}101 / 14712 \\
(0.7 \%)\end{array}$ & $\begin{array}{c}392 / 51993 \\
(0.8 \%)\end{array}$ & $\begin{array}{c}114 / 18701 \\
(0.6 \%)\end{array}$ & $\begin{array}{c}1290 / 142963 \\
(0.9 \%)\end{array}$ & $\begin{array}{c}233 / 25919 \\
(0.9 \%)\end{array}$ \\
\hline Missing & $\begin{array}{c}156154 / 254288 \\
(61 \%)\end{array}$ & $\begin{array}{c}9780 / 14712 \\
(66 \%)\end{array}$ & $\begin{array}{c}34211 / 51993 \\
(66 \%)\end{array}$ & $\begin{array}{c}9574 / 18701 \\
(51 \%)\end{array}$ & $\begin{array}{c}89509 / 142963 \\
(63 \%)\end{array}$ & $\begin{array}{c}13080 / 25919 \\
(50 \%)\end{array}$ \\
\hline
\end{tabular}

Hepatic disease, No. 


\begin{tabular}{|c|c|c|c|c|c|c|}
\hline No & $\begin{array}{c}95414 \text { / } 254288 \\
(38 \%)\end{array}$ & $\begin{array}{c}4807 / 14712 \\
(33 \%)\end{array}$ & $\begin{array}{c}17256 / 51993 \\
(33 \%)\end{array}$ & $\begin{array}{c}8957 / 18701 \\
(48 \%)\end{array}$ & $\begin{array}{c}51906 / 142963 \\
(36 \%)\end{array}$ & $\begin{array}{c}12488 / 25919 \\
(48 \%)\end{array}$ \\
\hline Yes & $\begin{array}{c}2395 / 254288 \\
(0.9 \%)\end{array}$ & $\begin{array}{c}106 / 14712 \\
(0.7 \%)\end{array}$ & $\begin{array}{c}475 / 51993 \\
(0.9 \%)\end{array}$ & $\begin{array}{c}150 / 18701 \\
(0.8 \%)\end{array}$ & $\begin{array}{c}1310 / 142963 \\
(0.9 \%)\end{array}$ & $\begin{array}{c}354 / 25919 \\
(1.4 \%)\end{array}$ \\
\hline Missing & $\begin{array}{c}156479 \text { / } 254288 \\
(62 \%)\end{array}$ & $\begin{array}{c}9799 / 14712 \\
(67 \%)\end{array}$ & $\begin{array}{c}34262 \text { / } 51993 \\
(66 \%)\end{array}$ & $\begin{array}{c}9594 / 18701 \\
(51 \%)\end{array}$ & $\begin{array}{c}89747 \text { / } 142963 \\
(63 \%)\end{array}$ & $\begin{array}{c}13077 \text { / } 25919 \\
(50 \%)\end{array}$ \\
\hline \multicolumn{7}{|c|}{ Puerperal ${ }^{a}$, No.· (\%) } \\
\hline No & $\begin{array}{c}44184 \text { / } 110722 \\
(40 \%)\end{array}$ & $\begin{array}{c}1966 / 5894 \\
(33 \%)\end{array}$ & $\begin{array}{c}7913 \text { / } 22987 \\
(34 \%)\end{array}$ & $\begin{array}{c}4100 / 7971 \\
(51 \%)\end{array}$ & $\begin{array}{c}24461 / 62605 \\
(39 \%)\end{array}$ & $\begin{array}{c}5744 / 11265 \\
(51 \%)\end{array}$ \\
\hline Yes & $\begin{array}{c}757 / 110722 \\
(0.7 \%)\end{array}$ & $\begin{array}{c}70 / 5894 \\
(1.2 \%)\end{array}$ & $\begin{array}{c}246 / 22987 \\
(1.1 \%)\end{array}$ & $76 / 7971$ (1.0\%) & $\begin{array}{c}318 / 62605 \\
(0.5 \%)\end{array}$ & $\begin{array}{c}47 / 11265 \\
(0.4 \%)\end{array}$ \\
\hline Missing & $\begin{array}{c}65781 / 110722 \\
(59 \%)\end{array}$ & $\begin{array}{c}3858 / 5894 \\
(65 \%)\end{array}$ & $\begin{array}{c}14828 / 22987 \\
(65 \%)\end{array}$ & $\begin{array}{c}3795 / 7971 \\
(48 \%)\end{array}$ & $\begin{array}{c}37826 / 62605 \\
(60 \%)\end{array}$ & $\begin{array}{c}5474 / 11265 \\
(49 \%)\end{array}$ \\
\hline \multicolumn{7}{|c|}{$\begin{array}{l}\text { Down syndrome, No. } \\
\text { (\%) }\end{array}$} \\
\hline No & $\begin{array}{c}97308 / 254288 \\
(38 \%)\end{array}$ & $\begin{array}{c}4874 \text { / } 14712 \\
(33 \%)\end{array}$ & $\begin{array}{c}17790 / 51993 \\
(34 \%)\end{array}$ & $\begin{array}{c}9094 / 18701 \\
(49 \%)\end{array}$ & $\begin{array}{c}52747 / 142963 \\
(37 \%)\end{array}$ & $\begin{array}{c}12803 / 25919 \\
(49 \%)\end{array}$ \\
\hline Yes & $\begin{array}{c}648 / 254288 \\
(0.3 \%)\end{array}$ & $\begin{array}{c}37 / 14712 \\
(0.3 \%)\end{array}$ & $\begin{array}{c}118 / 51993 \\
(0.2 \%)\end{array}$ & $\begin{array}{c}44 \text { / } 18701 \\
(0.2 \%)\end{array}$ & $\begin{array}{c}380 / 142963 \\
(0.3 \%)\end{array}$ & $\begin{array}{c}69 / 25919 \\
(0.3 \%)\end{array}$ \\
\hline Missing & $\begin{array}{c}156332 / 254288 \\
(61 \%)\end{array}$ & $\begin{array}{c}9801 / 14712 \\
(67 \%)\end{array}$ & $\begin{array}{c}34085 / 51993 \\
(66 \%)\end{array}$ & $\begin{array}{c}9563 / 18701 \\
(51 \%)\end{array}$ & $\begin{array}{c}89836 / 142963 \\
(63 \%)\end{array}$ & $\begin{array}{c}13047 \text { / } 25919 \\
(50 \%)\end{array}$ \\
\hline \multicolumn{7}{|c|}{ Other comorbidities, No. (\%) } \\
\hline No & $\begin{array}{c}50217 / 254288 \\
(20 \%)\end{array}$ & $\begin{array}{c}2682 / 14712 \\
(18 \%)\end{array}$ & $\begin{array}{c}8420 / 51993 \\
(16 \%)\end{array}$ & $\begin{array}{c}4376 / 18701 \\
(23 \%)\end{array}$ & $\begin{array}{c}28676 / 142963 \\
(20 \%)\end{array}$ & $\begin{array}{c}6063 / 25919 \\
(23 \%)\end{array}$ \\
\hline Yes & $\begin{array}{c}69893 / 254288 \\
(27 \%)\end{array}$ & $\begin{array}{c}3161 / 14712 \\
(21 \%)\end{array}$ & $\begin{array}{c}15041 / 51993 \\
(29 \%)\end{array}$ & $\begin{array}{c}5861 / 18701 \\
(31 \%)\end{array}$ & $\begin{array}{c}37538 / 142963 \\
(26 \%)\end{array}$ & $\begin{array}{c}8292 / 25919 \\
(32 \%)\end{array}$ \\
\hline Missing & $\begin{array}{c}134178 / 254288 \\
(53 \%)\end{array}$ & $\begin{array}{c}8869 / 14712 \\
(60 \%)\end{array}$ & $\begin{array}{c}28532 \text { / } 51993 \\
(55 \%)\end{array}$ & $\begin{array}{c}8464 \text { / } 18701 \\
(45 \%)\end{array}$ & $\begin{array}{c}76749 / 142963 \\
(54 \%)\end{array}$ & $\begin{array}{c}11564 \text { / } 25919 \\
(45 \%)\end{array}$ \\
\hline
\end{tabular}

ita from female patients

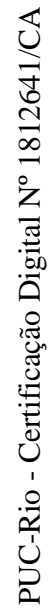


Table A12.16 - Times of the disease among patients with a defined hospital outcome (main analysis)

\begin{tabular}{|c|c|c|c|c|c|c|}
\hline & $\begin{array}{c}\text { Brazil } \\
(n=232,036)\end{array}$ & $\begin{array}{c}\text { North } \\
(n=13,496)\end{array}$ & $\begin{array}{l}\text { Northeast } \\
(n=45,238)\end{array}$ & $\begin{array}{l}\text { Central-West } \\
\qquad(n=17,012)\end{array}$ & $\begin{array}{l}\text { Southeast } \\
(n=131,556)\end{array}$ & $\begin{array}{c}\text { South } \\
(n=24,734)\end{array}$ \\
\hline \multicolumn{7}{|c|}{ Time from onset of symptoms, median (IQR) } \\
\hline $\begin{array}{l}\text { to hospital } \\
\text { admission [n = } \\
202842(87 \%)]\end{array}$ & $6(4,9)$ & $7(4,10)$ & $6(4,9)$ & $7(4,10)$ & $6(4,9)$ & $6(3,9)$ \\
\hline $\begin{array}{l}\text { to ICU admission [n } \\
=72154(91 \%)]\end{array}$ & $7(4,10)$ & $8(5,12)$ & $7(4,10)$ & $7(5,10)$ & $7(4,10)$ & $7(4,10)$ \\
\hline $\begin{array}{l}\text { to death [n = } 86482 \\
(99 \%)]\end{array}$ & $15(9,23)$ & $13(8,21)$ & $14(8,22)$ & $16(10,25)$ & $15(9,23)$ & $16(10,26)$ \\
\hline $\begin{array}{l}\text { Time from hospital } \\
\text { admission to death } \\
\text { [n=80527 (92\%)] }\end{array}$ & $10(5,17)$ & $7(3,14)$ & $9(4,16)$ & $11(5,19)$ & $10(5,18)$ & $12(6,20)$ \\
\hline
\end{tabular}

The numbers and proportions within brackets refer to the available data for each variable. ICU intensive care unit 
Table A12.17 - In-hospital mortality stratified by age and sex in Brazil

\begin{tabular}{lcc} 
& Total & By age \\
\hline Total & 232036 & $87515 / 232036(38 \%)$ \\
Age groups & & \\
$20-39$ & 30603 & $3780 / 30603(12 \%)$ \\
$40-49$ & 33968 & $6162 / 33968(18 \%)$ \\
$50-59$ & 43376 & $11818 / 43376(27 \%)$ \\
$60-69$ & 48270 & $20317 / 48270(42 \%)$ \\
$70-79$ & 41434 & $22651 / 41434(55 \%)$ \\
$80+$ & 34385 & $22787 / 34385(66 \%)$ \\
\hline
\end{tabular}

\begin{tabular}{lccc} 
& Total & Female & Male \\
\hline Total & 231995 & $36827 / 100826(37 \%)$ & $50676 / 131169(39 \%)$
\end{tabular}

Age groups

\begin{tabular}{llll}
$20-39$ & 30594 & $1577 / 13976(11 \%)$ & $2202 / 16618(13 \%)$ \\
$40-49$ & 33960 & $2249 / 12781(18 \%)$ & $3913 / 21179(18 \%)$ \\
$50-59$ & 43369 & $4374 / 17221(25 \%)$ & $7442 / 26148(28 \%)$ \\
$60-69$ & 48258 & $7838 / 20211(39 \%)$ & $12473 / 28047(44 \%)$ \\
$70-79$ & 41432 & $9337 / 18470(51 \%)$ & $13313 / 22962(58 \%)$ \\
$80+$ & 34382 & $11452 / 18167(63 \%)$ & $11333 / 16215(70 \%)$ \\
\hline
\end{tabular}



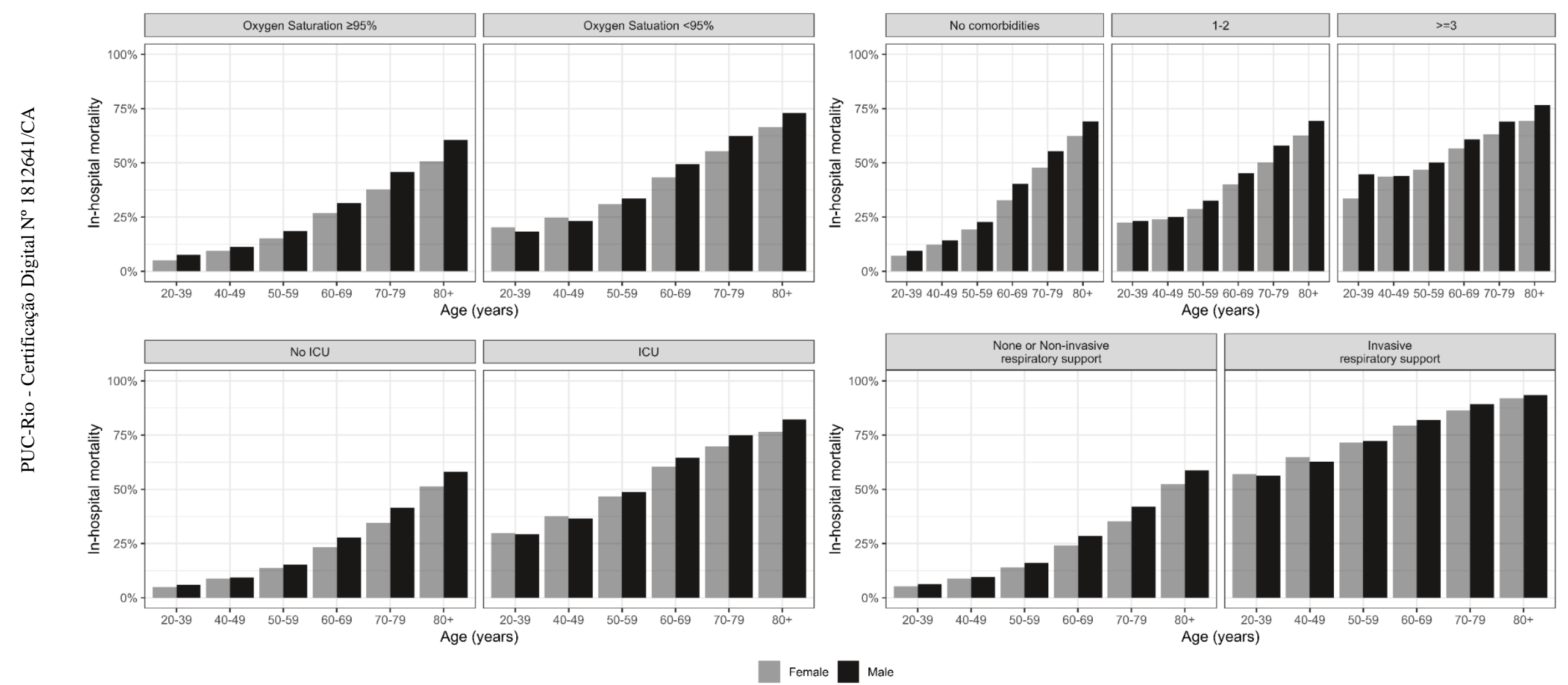

Figure A12.4 - In-hospital mortality stratified by age and sex accounting for the reported symptom of oxygen saturation < $95 \%$, number of comorbidities, ICU admission, and respiratory support. 
Table A12.18 - In-hospital mortality stratified by chronic comorbidities, level of education and selfreported race and age

\section{Comorbidities}

\begin{tabular}{lcccc} 
& Total & No Comorbidity & $1-2$ Comorbidities & $\geq 3$ Comorbidities \\
\hline $\begin{array}{l}\text { Total } \\
\text { Age groups }\end{array}$ & 84400 & $4494 / 13836(32 \%)$ & $26933 / 62766(43 \%)$ & $4685 / 7798(60 \%)$ \\
$20-39$ & 6780 & $291 / 2245(13 \%)$ & $937 / 4278(22 \%)$ & $104 / 257(40 \%)$ \\
$40-49$ & 9547 & $396 / 2171(18 \%)$ & $1591 / 6824(23 \%)$ & $243 / 552(44 \%)$ \\
$50-59$ & 15438 & $625 / 2691(23 \%)$ & $3432 / 11484(30 \%)$ & $594 / 1263(47 \%)$ \\
$60-69$ & 19886 & $951 / 2691(35 \%)$ & $6300 / 15112(42 \%)$ & $1187 / 2083(57 \%)$ \\
$70-79$ & 17957 & $1051 / 2165(49 \%)$ & $7360 / 13772(53 \%)$ & $1349 / 2020(67 \%)$ \\
$80+$ & 14792 & $1180 / 1873(63 \%)$ & $7313 / 11296(65 \%)$ & $1208 / 1623(74 \%)$ \\
\hline
\end{tabular}

\section{Self-reported race *}

\begin{tabular}{|c|c|c|c|c|c|}
\hline & Total & White & Black/Brown & Asian & Indigenous \\
\hline Total & 166882 & $30061 / 83423$ (36\%) & $34345 / 80392(43 \%)$ & $1031 / 2590(40 \%)$ & $202 / 477(42 \%)$ \\
\hline \multicolumn{6}{|c|}{ Age groups } \\
\hline 20-39 & 21677 & $1041 / 10493$ (10\%) & $1775 / 10817$ (16\%) & 43/300 (14\%) & $11 / 67(16 \%)$ \\
\hline $40-49$ & 23813 & $1758 / 11477$ (15\%) & $2784 / 11955$ (23\%) & $54 / 302$ (18\%) & $19 / 79$ (24\%) \\
\hline $50-59$ & 30866 & $3610 / 15171$ (24\%) & $5053 / 15175$ (33\%) & $122 / 428(29 \%)$ & $37 / 92(40 \%)$ \\
\hline $60-69$ & 34990 & 6703/17302 (39\%) & $8319 / 17043$ (49\%) & $221 / 554$ (40\%) & $31 / 91$ (34\%) \\
\hline $70-79$ & 30519 & $8027 / 15378$ (52\%) & $8773 / 14504(60 \%)$ & $289 / 561(52 \%)$ & $51 / 76(67 \%)$ \\
\hline $80+$ & 25017 & $8922 / 13602$ (66\%) & 7641/10898 (70\%) & $302 / 445(68 \%)$ & $53 / 72(74 \%)$ \\
\hline
\end{tabular}

${ }^{*}$ Race was collected as self-reported race or skin colour, originally classified as White (Branco), Black (Preto), Brown (Pardo), Asian (Amarelo), and Indigenous (Indígena)

\section{Level of education}

\begin{tabular}{|c|c|c|c|c|c|}
\hline & Total & Illiterate & Up to high school & High school & College/University \\
\hline Total & 79721 & $3146 / 4993$ (63\%) & $16489 / 35750$ (46\%) & $7735 / 26146(30 \%)$ & 2952/12832 (23\%) \\
\hline \multicolumn{6}{|c|}{ Age groups } \\
\hline $20-39$ & 11890 & 46/132 (35\%) & 451/2238 (20\%) & 755/6291 (12\%) & 235/3229 (7\%) \\
\hline $40-49$ & 12354 & 67/174 (39\%) & $884 / 3674$ (24\%) & 988/5715 (17\%) & 323/2791 (12\%) \\
\hline $50-59$ & 15279 & $168 / 385(44 \%)$ & $2081 / 6729(31 \%)$ & $1511 / 5592(27 \%)$ & $483 / 2573(19 \%)$ \\
\hline $60-69$ & 16216 & $513 / 885(58 \%)$ & 4107/8922 (46\%) & $1823 / 4265(43 \%)$ & 698/2144 (33\%) \\
\hline $70-79$ & 13405 & $939 / 1479(63 \%)$ & $4664 / 8075(58 \%)$ & $1474 / 2562(58 \%)$ & $646 / 1289(50 \%)$ \\
\hline $80+$ & 10577 & 1413/1938 (73\%) & $4302 / 6112(70 \%)$ & $1184 / 1721(69 \%)$ & $567 / 806(70 \%)$ \\
\hline
\end{tabular}


Table A12.19 - In-hospital mortality stratified by ICU admission, respiratory support and age

\begin{tabular}{lccc} 
& Total & No ICU admission & ICU admission \\
\hline $\begin{array}{l}\text { Total } \\
\text { Age groups }\end{array}$ & 205493 & $29361 / 125806(23 \%)$ & $47002 / 79687(59 \%)$ \\
$20-39$ & & & \\
$40-49$ & 27309 & $1088 / 19797(5 \%)$ & $2225 / 7512(30 \%)$ \\
$50-59$ & 30353 & $1910 / 20875(9 \%)$ & $3503 / 9478(37 \%)$ \\
$60-69$ & 38720 & $3634 / 24686(15 \%)$ & $6732 / 14034(48 \%)$ \\
$70-79$ & 42617 & $6350 / 24559(26 \%)$ & $11372 / 18058(63 \%)$ \\
$80+$ & 36423 & $7499 / 19575(38 \%)$ & $12257 / 16848(73 \%)$ \\
\hline
\end{tabular}

\begin{tabular}{lcccc} 
& Total & $\begin{array}{c}\text { No respiratory } \\
\text { support }\end{array}$ & $\begin{array}{c}\text { Non-invasive } \\
\text { ventilation }\end{array}$ & $\begin{array}{c}\text { Invasive } \\
\text { mechanical ventilation }\end{array}$ \\
\hline Total & 196248 & $8655 / 54314(16 \%)$ & $28287 / 96729(29 \%)$ & $36046 / 45205(80 \%)$ \\
Age groups & & & & \\
$20-39$ & 25871 & $396 / 11482(3 \%)$ & $929 / 11111(8 \%)$ & $1858 / 3278(57 \%)$ \\
$40-49$ & 28766 & $597 / 10170(6 \%)$ & $1663 / 14108(12 \%)$ & $2850 / 4488(64 \%)$ \\
$50-59$ & 37004 & $1085 / 10625(10 \%)$ & $3409 / 18793(18 \%)$ & $5459 / 7586(72 \%)$ \\
$60-69$ & 40811 & $1862 / 9467(20 \%)$ & $6031 / 20196(30 \%)$ & $9028 / 11148(81 \%)$ \\
$70-79$ & 34913 & $2225 / 7178(31 \%)$ & $7214 / 17029(42 \%)$ & $9433 / 10706(88 \%)$ \\
$80+$ & 28883 & $2490 / 5392(46 \%)$ & $9041 / 15492(58 \%)$ & $7418 / 7999(93 \%)$ \\
\hline
\end{tabular}

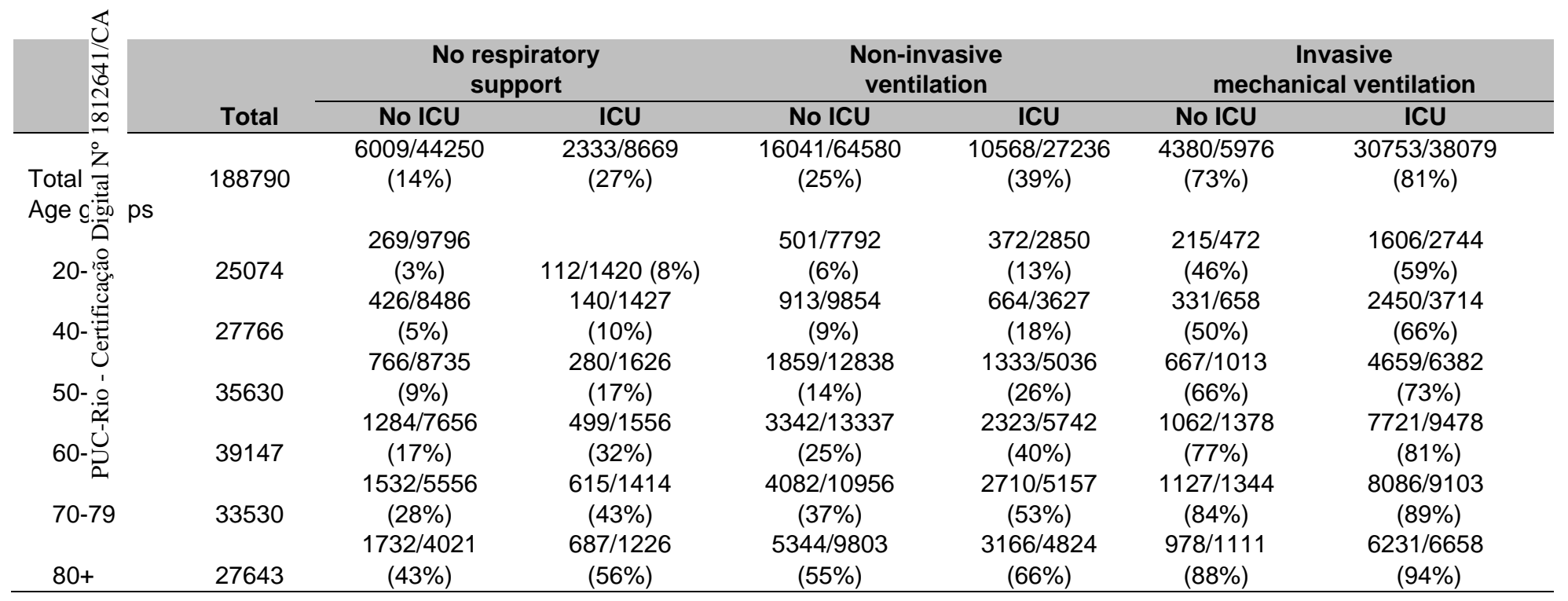



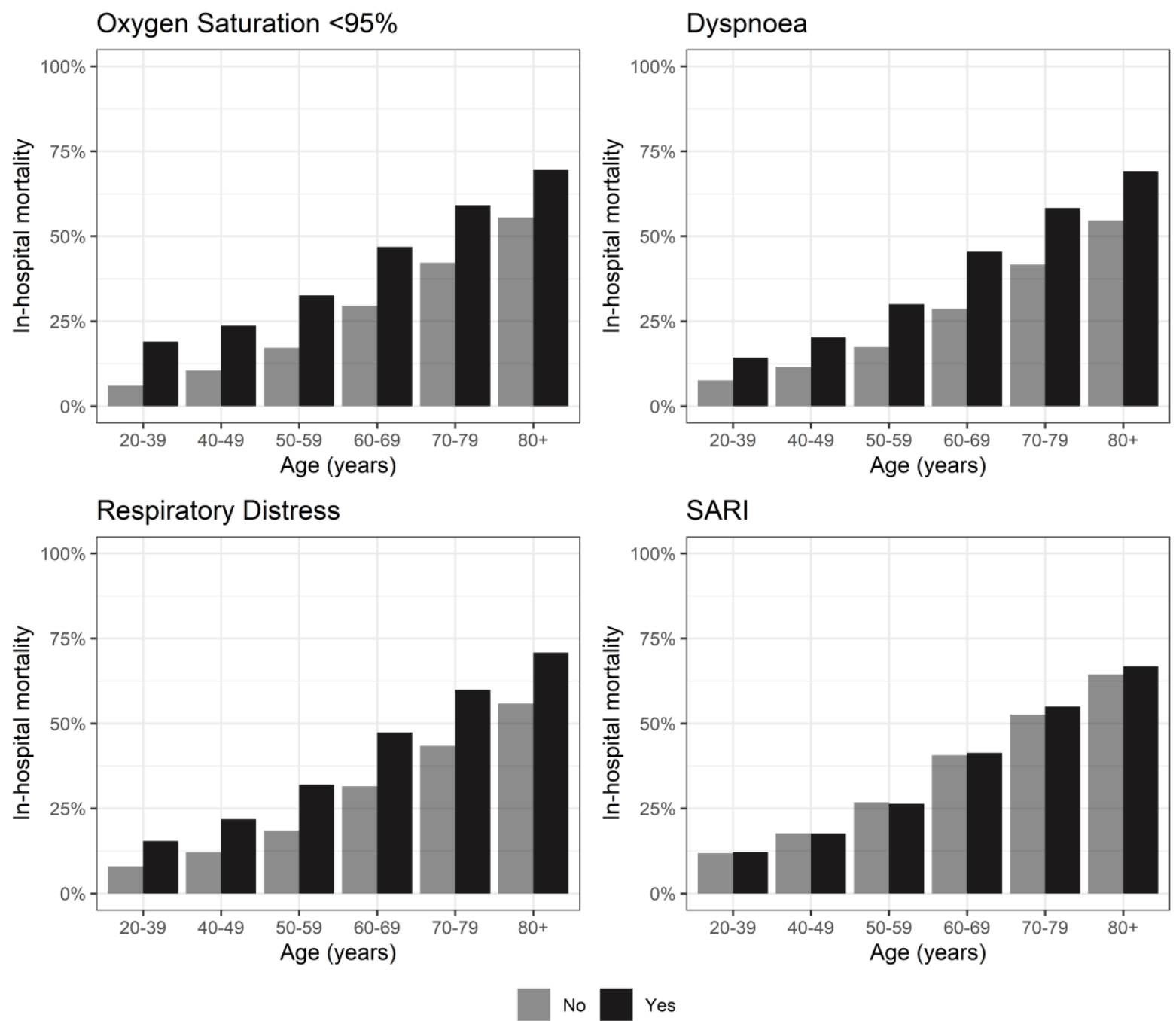

Figure A12.5 - In-hospital mortality per age group for symptoms of Oxygen saturation $<95 \%$, Dyspnoea, respiratory distress, and SARI diagnosis 
Table A12.20 - Health system burden in Brazil and its regions (number / per 100,000 inhabitants)

\section{Hospitalisations per population}

\begin{tabular}{|c|c|c|c|c|c|c|c|c|c|c|c|c|}
\hline & \multicolumn{2}{|l|}{ Brazil } & \multicolumn{2}{|l|}{ North } & \multicolumn{2}{|l|}{ Northeast } & \multicolumn{2}{|c|}{ Central-West } & \multicolumn{2}{|l|}{ Southeast } & \multicolumn{2}{|l|}{ South } \\
\hline Total & $232036 / 151778729$ & 152.9 & $13496 / 12049813$ & 112.0 & $45238 / 39882347$ & 113.4 & $17012 / 11678574$ & 145.7 & $131556 / 65803414$ & 199.9 & $24734 / 22364581$ & 110.6 \\
\hline Age groups & & & & & & & & & & & & \\
\hline $20-39$ & $603 / 68451093$ & 44.7 & $976 / 6448447$ & 30.6 & 3242 & 29.3 & $2498 / 5484644$ & 45.5 & 711 & 61.2 & $3372 / 9$ & 35.8 \\
\hline $40-49$ & $33968 / 29255478$ & 116.1 & $1973 / 2357103$ & 83.7 & $5575 / 7654000$ & 72.8 & $2795 / 2386731$ & 117.1 & $19901 / 12717264$ & 156.5 & $3724 / 4140380$ & 89.9 \\
\hline $50-59$ & $43376 / 23875081$ & 181.7 & $2272 / 1600270$ & 142.0 & $7461 / 5930317$ & 125.8 & $3373 / 1825822$ & 184.7 & $25389 / 10724660$ & 236.7 & $4881 / 3794012$ & 128.7 \\
\hline $60-69$ & 4827 & 288.5 & 2816 & 288.9 & & 236.1 & 343 & 297.3 & $27453 /$ & 346.7 & $5370 / 278$ & 192.5 \\
\hline $70-79$ & $41434 / 9023052$ & 459.2 & $2616 / 470277$ & 556.3 & $9086 / 2245607$ & 404.6 & 2823 & 490.8 & $22658 / 4225114$ & 536.3 & $4251 / 1506892$ & 282.1 \\
\hline $80+$ & $34385 / 4441053$ & 774.3 & $1843 / 198888$ & 926.7 & $8334 / 1110376$ & 750.6 & $2087 / 250358$ & 833.6 & $18985 / 2157323$ & 880.0 & $3136 / 724108$ & 433.1 \\
\hline
\end{tabular}

\section{ICU admissions per population}

\begin{tabular}{|c|c|c|c|c|c|c|c|c|c|c|c|c|}
\hline & \multicolumn{2}{|l|}{ Brazil } & \multicolumn{2}{|l|}{ North } & \multicolumn{2}{|l|}{ Northeast } & \multicolumn{2}{|c|}{ Central-West } & \multicolumn{2}{|c|}{ Southeast } & \multicolumn{2}{|l|}{ South } \\
\hline Total & $79687 / 151778729$ & 52.5 & $3786 / 12049813$ & 31.4 & $14867 / 39882347$ & 37.3 & $6682 / 11678574$ & 57.2 & $45224 / 65803414$ & 68.7 & $9128 / 22364581$ & 40.8 \\
\hline Age grou & & & & & & & & & & & & \\
\hline $20-39$ & $7512 / 68451093$ & 11.0 & $334 / 6448447$ & 5.2 & $1279 / 19048242$ & 6.7 & $732 / 5484644$ & 13.3 & $4354 / 28059711$ & 15.5 & $813 / 9410049$ & 8.6 \\
\hline $40-49$ & $9478 / 29255478$ & 32.4 & $404 / 2357103$ & 17.1 & $1440 / 7654000$ & 18.8 & $890 / 2386731$ & 37.3 & $5630 / 12717264$ & 44.3 & $1114 / 4140380$ & 26.9 \\
\hline $50-59$ & $14034 / 23875081$ & 58.8 & $645 / 1600270$ & 40.3 & $2324 / 5930317$ & 39.2 & $1266 / 1825822$ & 69.3 & $8097 / 10724660$ & 75.5 & $1702 / 3794012$ & 44.9 \\
\hline $60-69$ & $18058 / 16732972$ & 107.9 & $950 / 974828$ & 97.5 & $3318 / 3893805$ & 85.2 & $1420 / 1155857$ & 122.9 & $10094 / 7919342$ & 127.5 & $2276 / 2789140$ & 81.6 \\
\hline $70-79$ & $16848 / 9023052$ & 186.7 & $891 / 470277$ & 189.5 & $3401 / 2245607$ & 151.5 & $1345 / 575162$ & 233.8 & $9264 / 4225114$ & 219.3 & $1947 / 1506892$ & 129.2 \\
\hline $80+$ & $13757 / 4441053$ & 309.8 & $562 / 198888$ & 282.6 & $3105 / 1110376$ & 279.6 & $1029 / 250358$ & 411.0 & $7785 / 2157323$ & 360.9 & $1276 / 724108$ & 176.2 \\
\hline
\end{tabular}

\section{Hospitalisations requiring invasive mechanical ventilation per population}

\begin{tabular}{|c|c|c|c|c|c|c|c|c|c|c|c|c|}
\hline & \multicolumn{2}{|l|}{ Brazil } & \multicolumn{2}{|l|}{ North } & \multicolumn{2}{|c|}{ Northeast } & \multicolumn{2}{|c|}{ Central-West } & \multicolumn{2}{|c|}{ Southeast } & \multicolumn{2}{|l|}{ South } \\
\hline Total & $45205 / 151778729$ & 29.8 & $3155 / 12049813$ & 26.2 & $10322 / 39882347$ & 25.9 & $3667 / 11678574$ & 31.4 & $22648 / 65803414$ & 34.4 & $5413 / 22364581$ & 24.2 \\
\hline Age grou & & & & & & & & & & & & \\
\hline $20-39$ & $3278 / 68451093$ & 4.8 & $250 / 6448447$ & 3.9 & $791 / 19048242$ & 4.2 & $279 / 5484644$ & 5.1 & $1559 / 28059711$ & 5.6 & $399 / 9410049$ & 4.2 \\
\hline $40-49$ & $4488 / 29255478$ & 15.3 & $334 / 2357103$ & 14.2 & $947 / 7654000$ & 12.4 & $397 / 2386731$ & 16.6 & $2239 / 12717264$ & 17.6 & $571 / 4140380$ & 13.8 \\
\hline $50-59$ & $7586 / 23875081$ & 31.8 & $520 / 1600270$ & 32.5 & $1579 / 5930317$ & 26.6 & $605 / 1825822$ & 33.1 & $3923 / 10724660$ & 36.6 & $959 / 3794012$ & 25.3 \\
\hline $60-69$ & $11148 / 16732972$ & 66.6 & $821 / 974828$ & 84.2 & $2353 / 3893805$ & 60.4 & $875 / 1155857$ & 75.7 & $5673 / 7919342$ & 71.6 & $1426 / 2789140$ & 51.1 \\
\hline $70-79$ & $10706 / 9023052$ & 118.7 & $765 / 470277$ & 162.7 & $2494 / 2245607$ & 111.1 & $863 / 575162$ & 150.0 & $5291 / 4225114$ & 125.2 & $1293 / 1506892$ & 85.8 \\
\hline $80+$ & $7999 / 4441053$ & 180.1 & $465 / 198888$ & 233.8 & $2158 / 1110376$ & 194.3 & $648 / 250358$ & 258.8 & $3963 / 2157323$ & 183.7 & $765 / 724108$ & 105.6 \\
\hline
\end{tabular}


Table A12.21 - Overall in-hospital mortality, among those admitted to the ICU or under invasive mechanical ventilation, stratified by age and Brazilian regions.

\section{In-hospital mortality (Overall)}

\begin{tabular}{|c|c|c|c|c|c|c|}
\hline & Brazil & North & Northeast & Central-West & Southeast & South \\
\hline Total & $87515 / 232036(37.7 \%)$ & $6727 / 13496(49.8 \%)$ & $21858 / 45238(48.3 \%)$ & $5964 / 17012(35.1 \%)$ & $45269 / 131556(34.4 \%)$ & $7697 / 24734(31.1 \%)$ \\
\hline \multicolumn{7}{|c|}{ Age groups } \\
\hline $20-39$ & $3780 / 30603(12.4 \%)$ & $393 / 1976(19.9 \%)$ & $1083 / 5587(19.4 \%)$ & $284 / 2498(11.4 \%)$ & $1736 / 17170(10.1 \%)$ & $284 / 3372(8.4 \%)$ \\
\hline $40-49$ & $6162 / 33968(18.1 \%)$ & $556 / 1973(28.2 \%)$ & $1542 / 5575(27.7 \%)$ & $504 / 2795(18 \%)$ & $3062 / 19901(15.4 \%)$ & 498/3724 (13.4\%) \\
\hline $50-59$ & $11818 / 43376(27.2 \%)$ & 945/2272 (41.6\%) & $2893 / 7461(38.8 \%)$ & $863 / 3373(25.6 \%)$ & $6119 / 25389(24.1 \%)$ & $998 / 4881(20.4 \%)$ \\
\hline $60-69$ & $20317 / 48270(42.1 \%)$ & $1662 / 2816(59 \%)$ & $4730 / 9195(51.4 \%)$ & $1380 / 3436(40.2 \%)$ & $10659 / 27453(38.8 \%)$ & $1886 / 5370(35.1 \%)$ \\
\hline $70-79$ & $22651 / 41434(54.7 \%)$ & $1784 / 2616(68.2 \%)$ & $5660 / 9086(62.3 \%)$ & $1528 / 2823(54.1 \%)$ & $11583 / 22658(51.1 \%)$ & $2096 / 4251(49.3 \%)$ \\
\hline $80+$ & $22787 / 34385(66.3 \%)$ & $1387 / 1843(75.3 \%)$ & $5950 / 8334(71.4 \%)$ & $1405 / 2087(67.3 \%)$ & $12110 / 18985(63.8 \%)$ & $1935 / 3136(61.7 \%)$ \\
\hline
\end{tabular}

\section{In-hospital mortality (ICU admissions)}

\begin{tabular}{|c|c|c|c|c|c|c|}
\hline & Brazil & North & $\begin{array}{c}\text { Northeast } \\
\end{array}$ & Central-West & Southeast & South \\
\hline Total & $47002 / 79687(59 \%)$ & $3022 / 3786(79.8 \%)$ & $10483 / 14867(70.5 \%)$ & $3734 / 6682(55.9 \%)$ & $24693 / 45224(54.6 \%)$ & $5070 / 9128(55.5 \%)$ \\
\hline \multicolumn{7}{|c|}{ Age groups } \\
\hline $20-39$ & $2225 / 7512(29.6 \%)$ & $195 / 334(58.4 \%)$ & $579 / 1279(45.3 \%)$ & $185 / 732(25.3 \%)$ & $1065 / 4354(24.5 \%)$ & $201 / 813(24.7 \%)$ \\
\hline $40-49$ & $3503 / 9478(37 \%)$ & $265 / 404(65.6 \%)$ & $799 / 1440(55.5 \%)$ & $324 / 890(36.4 \%)$ & $1763 / 5630(31.3 \%)$ & $352 / 1114(31.6 \%)$ \\
\hline $50-59$ & $6732 / 14034(48 \%)$ & $468 / 645(72.6 \%)$ & $1441 / 2324(62 \%)$ & $554 / 1266(43.8 \%)$ & $3513 / 8097$ (43.4\%) & $756 / 1702(44.4 \%)$ \\
\hline $60-69$ & $11372 / 18058(63 \%)$ & $800 / 950(84.2 \%)$ & $2350 / 3318(70.8 \%)$ & $896 / 1420(63.1 \%)$ & $5980 / 10094(59.2 \%)$ & $1346 / 2276(59.1 \%)$ \\
\hline $70-79$ & $12257 / 16848(72.8 \%)$ & $779 / 891(87.4 \%)$ & $2720 / 3401(80 \%)$ & $954 / 1345(70.9 \%)$ & $6408 / 9264(69.2 \%)$ & $1396 / 1947(71.7 \%)$ \\
\hline $80+$ & $10913 / 13757(79.3 \%)$ & $515 / 562(91.6 \%)$ & $2594 / 3105(83.5 \%)$ & $821 / 1029(79.8 \%)$ & $5964 / 7785(76.6 \%)$ & 1019/1276 (79.9\%) \\
\hline
\end{tabular}

\section{In-hospital mortality (Invasive mechanical ventilation)}

\begin{tabular}{|c|c|c|c|c|c|c|}
\hline & Brazil & North & Northeast & Central-West & Southeast & South \\
\hline Total & $36046 / 45205(79.7 \%)$ & $2810 / 3155(89.1 \%)$ & $8963 / 10322(86.8 \%)$ & $3039 / 3667(82.9 \%)$ & $17325 / 22648(76.5 \%)$ & $3909 / 5413(72.2 \%)$ \\
\hline \multicolumn{7}{|c|}{ Age groups } \\
\hline $20-39$ & $1858 / 3278(56.7 \%)$ & $193 / 250(77.2 \%)$ & $551 / 791(69.7 \%)$ & $164 / 279(58.8 \%)$ & $774 / 1559(49.6 \%)$ & 176/399 (44.1\%) \\
\hline $40-49$ & $2850 / 4488(63.5 \%)$ & $269 / 334(80.5 \%)$ & $717 / 947(75.7 \%)$ & $273 / 397(68.8 \%)$ & $1317 / 2239(58.8 \%)$ & $274 / 571(48 \%)$ \\
\hline $50-59$ & $5459 / 7586(72 \%)$ & $446 / 520(85.8 \%)$ & $1291 / 1579(81.8 \%)$ & $456 / 605(75.4 \%)$ & $2662 / 3923(67.9 \%)$ & $604 / 959(63 \%)$ \\
\hline $60-69$ & $9028 / 11148(81 \%)$ & $748 / 821(91.1 \%)$ & $2056 / 2353(87.4 \%)$ & $743 / 875(84.9 \%)$ & $4424 / 5673(78 \%)$ & $1057 / 1426(74.1 \%)$ \\
\hline $70-79$ & $9433 / 10706(88.1 \%)$ & $711 / 765(92.9 \%)$ & $2289 / 2494(91.8 \%)$ & 787/863 (91.2\%) & $4551 / 5291(86 \%)$ & $1095 / 1293(84.7 \%)$ \\
\hline $80+$ & $7418 / 7999(92.7 \%)$ & $443 / 465(95.3 \%)$ & $2059 / 2158(95.4 \%)$ & $616 / 648(95.1 \%)$ & $3597 / 3963(90.8 \%)$ & $703 / 765(91.9 \%)$ \\
\hline
\end{tabular}


Table A12.22 - Hospitalisations and ICU admissions per hospital and ICU beds in Brazil and regions*

\begin{tabular}{|c|c|c|c|c|c|c|c|c|c|c|c|c|}
\hline & \multicolumn{2}{|c|}{ Brazil } & \multicolumn{2}{|c|}{ North } & \multicolumn{2}{|c|}{ Northeast } & \multicolumn{2}{|c|}{ Central-West } & \multicolumn{2}{|c|}{ Southeast } & \multicolumn{2}{|c|}{ South } \\
\hline & $\mathrm{N}$ & Rate & $\mathrm{N}$ & Rate & $\mathrm{N}$ & Rate & $\mathrm{N}$ & Rate & $\mathrm{N}$ & Rate & $\mathrm{N}$ & Rate \\
\hline $\begin{array}{c}\text { All } \\
\text { hospitalizations } \\
\text { (n/per 100,000 } \\
\text { hospital beds) }\end{array}$ & $\begin{array}{l}232036 / \\
356344\end{array}$ & 65116 & $\begin{array}{l}13496 / \\
23719\end{array}$ & 56900 & $\begin{array}{l}45238 / \\
87604\end{array}$ & 51639 & $\begin{array}{l}17012 / \\
29606\end{array}$ & 57461 & $\begin{array}{c}131556 / \\
157510\end{array}$ & 83522 & $\begin{array}{l}24734 / \\
57905\end{array}$ & 42715 \\
\hline $\begin{array}{l}\text { ICU admissions } \\
\text { (n/per } 1,000 \\
\text { ICU beds) }\end{array}$ & $\begin{array}{c}79687 / \\
37692\end{array}$ & 2114 & $\begin{array}{c}3786 / \\
1686\end{array}$ & 2246 & $\begin{array}{c}14867 / \\
7171\end{array}$ & 2073 & $\begin{array}{l}6682 / \\
3340\end{array}$ & 2001 & $\begin{array}{l}45224 / \\
20403\end{array}$ & 2217 & $\begin{array}{l}9128 / \\
5092\end{array}$ & 1793 \\
\hline
\end{tabular}

* Beds data on February 2020. 

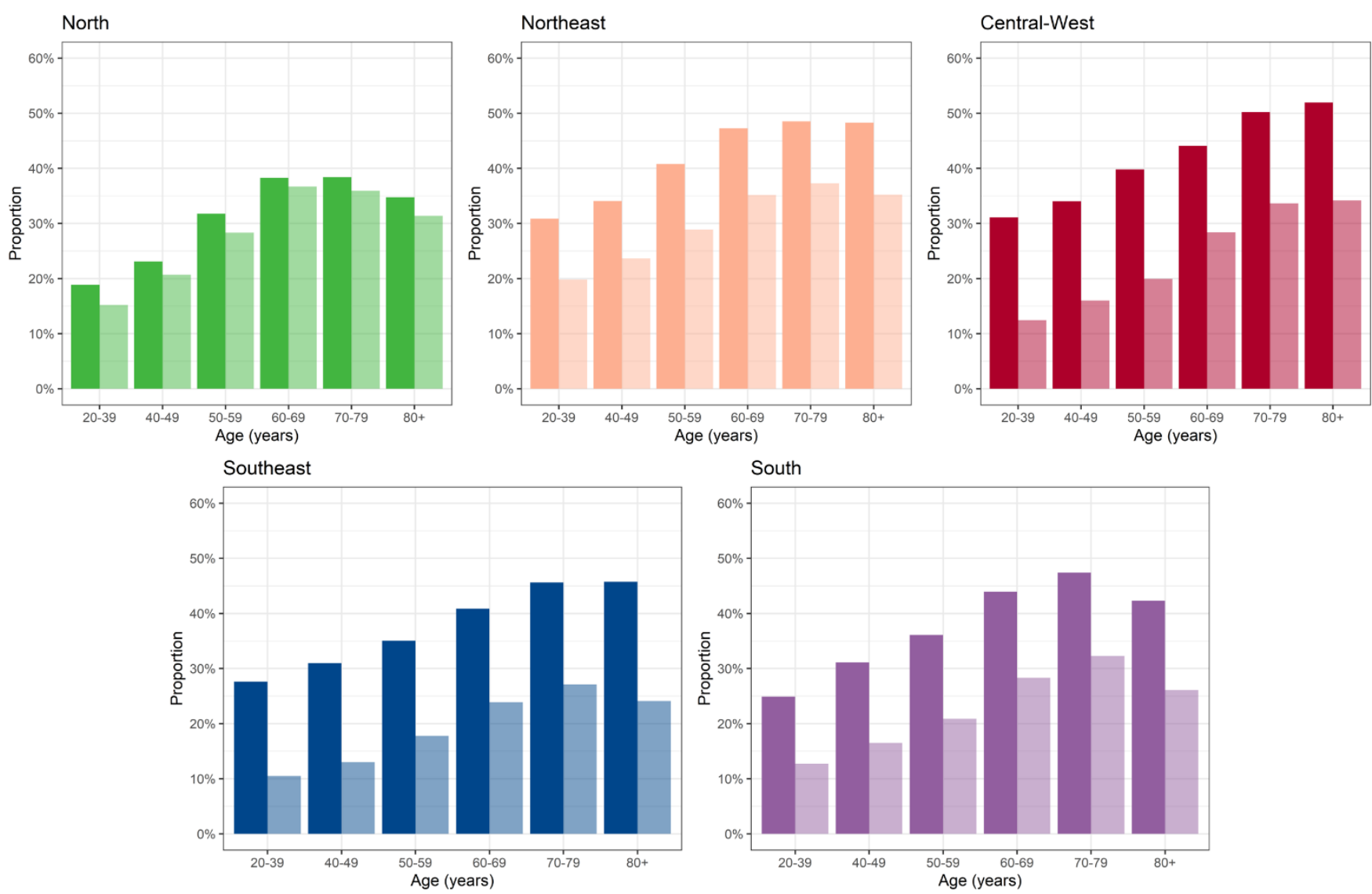

ICU Invasive ventilation

Figure A12.6 - Proportion of intensive care unit admission and use of invasive mechanical ventilation stratified by age in hospitalised COVID-19 patients in the five regions of Brazil. 
Table A12.23 - Use of resources in terms of proportions of hospitalised patients admitted to the ICU and under invasive mechanical ventilation stratified by age and region

\section{Proportion of ICU admission}

\begin{tabular}{|c|c|c|c|c|c|c|}
\hline & Brazil & North & Northeast & Central-West & Southeast & South \\
\hline Total & $\begin{array}{c}79687 / 205493 \\
(38.8 \%)\end{array}$ & $\begin{array}{c}3786 / 11973 \\
(31.6 \%)\end{array}$ & $\begin{array}{c}14867 / 34532 \\
(43.1 \%)\end{array}$ & $\begin{array}{c}6682 / 16035 \\
(41.7 \%)\end{array}$ & $\begin{array}{c}45224 / 119083 \\
(38 \%)\end{array}$ & $\begin{array}{c}9128 / 23870 \\
(38.2 \%)\end{array}$ \\
\hline \multicolumn{7}{|l|}{$\begin{array}{l}\text { Age } \\
\text { groups }\end{array}$} \\
\hline $20-39$ & $\begin{array}{c}7512 / 27309 \\
(27.5 \%)\end{array}$ & $\begin{array}{c}334 / 1773 \\
(18.8 \%)\end{array}$ & $\begin{array}{c}1279 / 4144 \\
(30.9 \%)\end{array}$ & $\begin{array}{c}732 / 2354 \\
(31.1 \%)\end{array}$ & $\begin{array}{c}4354 / 15772 \\
(27.6 \%)\end{array}$ & $\begin{array}{c}813 / 3266 \\
(24.9 \%)\end{array}$ \\
\hline $40-49$ & $\begin{array}{c}9478 / 30353 \\
(31.2 \%)\end{array}$ & $\begin{array}{c}404 / 1748 \\
(23.1 \%)\end{array}$ & $\begin{array}{c}1440 / 4225 \\
(34.1 \%)\end{array}$ & $\begin{array}{c}890 / 2618 \\
(34 \%)\end{array}$ & $\begin{array}{c}5630 / 18181 \\
(31 \%)\end{array}$ & $\begin{array}{c}1114 / 3581 \\
(31.1 \%)\end{array}$ \\
\hline $50-59$ & $\begin{array}{c}14034 / 38720 \\
(36.2 \%)\end{array}$ & $\begin{array}{c}645 / 2031 \\
(31.8 \%)\end{array}$ & $\begin{array}{c}2324 / 5698 \\
(40.8 \%)\end{array}$ & $\begin{array}{c}1266 / 3182 \\
(39.8 \%)\end{array}$ & $\begin{array}{c}8097 / 23092 \\
(35.1 \%)\end{array}$ & $\begin{array}{c}1702 / 4717 \\
(36.1 \%)\end{array}$ \\
\hline $60-69$ & $\begin{array}{c}18058 / 42617 \\
(42.4 \%)\end{array}$ & $\begin{array}{c}950 / 2482 \\
(38.3 \%)\end{array}$ & $\begin{array}{c}3318 / 7024 \\
(47.2 \%)\end{array}$ & $\begin{array}{c}1420 / 3221 \\
(44.1 \%)\end{array}$ & $\begin{array}{c}10094 / 24709 \\
(40.9 \%)\end{array}$ & $\begin{array}{c}2276 / 5181 \\
(43.9 \%)\end{array}$ \\
\hline $70-79$ & $\begin{array}{c}16848 / 36423 \\
(46.3 \%)\end{array}$ & $\begin{array}{c}891 / 2322 \\
(38.4 \%)\end{array}$ & $\begin{array}{c}3401 / 7010 \\
(48.5 \%)\end{array}$ & $\begin{array}{c}1345 / 2679 \\
(50.2 \%)\end{array}$ & $\begin{array}{c}9264 / 20304 \\
(45.6 \%)\end{array}$ & $\begin{array}{c}1947 / 4108 \\
(47.4 \%)\end{array}$ \\
\hline $80_{+}$ & $\begin{array}{c}13757 / 30071 \\
(45.7 \%)\end{array}$ & $\begin{array}{c}562 / 1617 \\
(34.8 \%)\end{array}$ & $\begin{array}{c}3105 / 6431 \\
(48.3 \%)\end{array}$ & $\begin{array}{c}1029 / 1981 \\
(51.9 \%)\end{array}$ & $\begin{array}{c}7785 / 17025 \\
(45.7 \%)\end{array}$ & $\begin{array}{c}1276 / 3017 \\
(42.3 \%)\end{array}$ \\
\hline
\end{tabular}

\section{Proportion on invasive mechanical ventilation}

\begin{tabular}{|c|c|c|c|c|c|c|}
\hline & Brazil & North & Northeast & $\begin{array}{l}\text { Central- } \\
\text { West }\end{array}$ & Southeast & South \\
\hline Total & $\begin{array}{c}45205 / 196248 \\
(23 \%)\end{array}$ & $\begin{array}{c}3155 / 10945 \\
(28.8 \%)\end{array}$ & $\begin{array}{c}10322 / 32984 \\
(31.3 \%)\end{array}$ & $\begin{array}{c}3667 / 15304 \\
(24 \%)\end{array}$ & $\begin{array}{c}22648 / 113848 \\
(19.9 \%)\end{array}$ & $\begin{array}{c}5413 / 23167 \\
(23.4 \%)\end{array}$ \\
\hline \multicolumn{7}{|l|}{$\begin{array}{l}\text { Age } \\
\text { groups }\end{array}$} \\
\hline 20-39 & $\begin{array}{c}3278 / 25871 \\
(12.7 \%) \\
\end{array}$ & $\begin{array}{c}250 / 1644 \\
(15.2 \%)\end{array}$ & $\begin{array}{c}791 / 3995 \\
(19.8 \%) \\
\end{array}$ & $\begin{array}{c}279 / 2245 \\
(12.4 \%) \\
\end{array}$ & $\begin{array}{c}1559 / 14846 \\
(10.5 \%) \\
\end{array}$ & $\begin{array}{c}399 / 3141 \\
(12.7 \%) \\
\end{array}$ \\
\hline $40-49$ & $\begin{array}{c}4488 / 28766 \\
(15.6 \%)\end{array}$ & $\begin{array}{c}334 / 1616 \\
(20.7 \%)\end{array}$ & $\begin{array}{c}947 / 4005 \\
(23.6 \%) \\
\end{array}$ & $\begin{array}{c}397 / 2479 \\
(16 \%)\end{array}$ & $\begin{array}{c}2239 / 17206 \\
(13 \%)\end{array}$ & $\begin{array}{c}571 / 3460 \\
(16.5 \%)\end{array}$ \\
\hline $50-59$ & $\begin{array}{c}7586 / 37004 \\
(20.5 \%)\end{array}$ & $\begin{array}{c}520 / 1836 \\
(28.3 \%)\end{array}$ & $\begin{array}{c}1579 / 5468 \\
(28.9 \%) \\
\end{array}$ & $\begin{array}{c}605 / 3035 \\
(19.9 \%)\end{array}$ & $\begin{array}{c}3923 / 22070 \\
(17.8 \%)\end{array}$ & $\begin{array}{c}959 / 4595 \\
(20.9 \%) \\
\end{array}$ \\
\hline $60-69$ & $\begin{array}{c}11148 / 40811 \\
(27.3 \%)\end{array}$ & $\begin{array}{c}821 / 2237 \\
(36.7 \%)\end{array}$ & $\begin{array}{c}2353 / 6699 \\
(35.1 \%)\end{array}$ & $\begin{array}{c}875 / 3082 \\
(28.4 \%)\end{array}$ & $\begin{array}{c}5673 / 23756 \\
(23.9 \%)\end{array}$ & $\begin{array}{c}1426 / 5037 \\
(28.3 \%)\end{array}$ \\
\hline 70-79 & $\begin{array}{c}10706 / 34913 \\
(30.7 \%)\end{array}$ & $\begin{array}{c}765 / 2130 \\
(35.9 \%)\end{array}$ & $\begin{array}{c}2494 / 6690 \\
(37.3 \%)\end{array}$ & $\begin{array}{c}863 / 2566 \\
(33.6 \%)\end{array}$ & $\begin{array}{c}5291 / 19524 \\
(27.1 \%)\end{array}$ & $\begin{array}{c}1293 / 4003 \\
(32.3 \%)\end{array}$ \\
\hline $80+$ & $\begin{array}{c}7999 / 28883 \\
(27.7 \%)\end{array}$ & $\begin{array}{c}465 / 1482 \\
(31.4 \%)\end{array}$ & $\begin{array}{c}2158 / 6127 \\
(35.2 \%)\end{array}$ & $\begin{array}{c}648 / 1897 \\
(34.2 \%)\end{array}$ & $\begin{array}{c}3963 / 16446 \\
(24.1 \%)\end{array}$ & $\begin{array}{c}765 / 2931 \\
(26.1 \%)\end{array}$ \\
\hline
\end{tabular}


12.3.4

\section{Appendix A4.4 - Sensitivity analyses}

Table A12.24 - Patients characteristics stratified by region (Sensitivity Analysis: patients with laboratorial and clinical diagnosis of COVID-19)

\begin{tabular}{|c|c|c|c|c|c|c|}
\hline Variables & $\begin{array}{c}\text { Brazil } \\
(n=314,615)\end{array}$ & $\begin{array}{c}\text { North } \\
(n=27,502)\end{array}$ & $\begin{array}{l}\text { Northeast } \\
(n=71,442)\end{array}$ & $\begin{array}{c}\text { Central-West } \\
(n=23,908)\end{array}$ & $\begin{array}{c}\text { Southeast } \\
(n=162,563)\end{array}$ & $\begin{array}{c}\text { South } \\
(n=29,200)\end{array}$ \\
\hline $\begin{array}{l}\text { Age, mean }(\mathrm{sd})[\mathrm{n}=314615(100 \%)] \\
\text { median }(\mathrm{IQR})\end{array}$ & $\begin{array}{c}60(17) \\
61(48,73)\end{array}$ & $\begin{array}{c}59(18) \\
60(46,73)\end{array}$ & $\begin{array}{c}62(18) \\
64(49,76)\end{array}$ & $\begin{array}{c}58(17) \\
58(46,71)\end{array}$ & $\begin{array}{c}60(17) \\
61(48,73)\end{array}$ & $\begin{array}{c}59(17) \\
60(47,72)\end{array}$ \\
\hline \multicolumn{7}{|l|}{ Age group, $\mathrm{N}(\%)$} \\
\hline $40-49$ & $45773(15 \%)$ & $4063(15 \%)$ & $8992(13 \%)$ & $3967(17 \%)$ & $24312(15 \%)$ & $4439(15 \%)$ \\
\hline $50-59$ & $58569(19 \%)$ & $4697(17 \%)$ & $11893(17 \%)$ & $4832(20 \%)$ & $31330(19 \%)$ & $5817(20 \%)$ \\
\hline $60-69$ & $65266(21 \%)$ & $5603(20 \%)$ & $14359(20 \%)$ & $4785(20 \%)$ & $34158(21 \%)$ & $6361(22 \%)$ \\
\hline $70-79$ & $56297(18 \%)$ & $5011(18 \%)$ & $14366(20 \%)$ & $3880(16 \%)$ & $28105(17 \%)$ & $4935(17 \%)$ \\
\hline $80+$ & $46320(15 \%)$ & $3601(13 \%)$ & $12631(18 \%)$ & $2802(12 \%)$ & $23639(15 \%)$ & 3647 (12\%) \\
\hline Male sex, No $(\%)[n=314556(100 \%)]$ & $177819(57 \%)$ & $16317(59 \%)$ & $39937(56 \%)$ & $13710(57 \%)$ & $91307(56 \%)$ & $16548(57 \%)$ \\
\hline \multicolumn{7}{|l|}{ Self-reported race, No. (\%) $[n=229079(73 \%)]^{a}$} \\
\hline White & $104274(46 \%)$ & $2419(10 \%)$ & $7292(15 \%)$ & $4349(29 \%)$ & $67763(57 \%)$ & $22451(88 \%)$ \\
\hline Black/Brown & $120326(53 \%)$ & $19877(86 \%)$ & $38811(82 \%)$ & $10071(67 \%)$ & $48864(41 \%)$ & $2703(11 \%)$ \\
\hline Asian & $3511(1.5 \%)$ & $333(1.4 \%)$ & $908(1.9 \%)$ & $310(2.1 \%)$ & $1802(1.5 \%)$ & $158(0.6 \%)$ \\
\hline Indigenous & $968(0.4 \%)$ & $447(1.9 \%)$ & $139(0.3 \%)$ & $225(1.5 \%)$ & $98(<0.1 \%)$ & $59(0.2 \%)$ \\
\hline \multicolumn{7}{|l|}{ Level of education, No. (\%) $[n=109128(35 \%)]$} \\
\hline Illiterate & $8084(7.4 \%)$ & $1734(13 \%)$ & $2728(15 \%)$ & $396(5.7 \%)$ & $2682(4.7 \%)$ & $544(4.0 \%)$ \\
\hline Up to high school & $49609(45 \%)$ & $6131(45 \%)$ & 7790 (44\%) & 2926 (42\%) & $25914(45 \%)$ & $6848(51 \%)$ \\
\hline High school & $34909(32 \%)$ & $4274(31 \%)$ & 4995 (28\%) & $2371(34 \%)$ & $19312(34 \%)$ & 3957 (29\%) \\
\hline College/University & $16526(15 \%)$ & $1620(12 \%)$ & $2334(13 \%)$ & $1271(18 \%)$ & $9184(16 \%)$ & $2117(16 \%)$ \\
\hline 0 & $18705(17 \%)$ & $1675(20 \%)$ & $3846(17 \%)$ & $2088(20 \%)$ & 8928 (16\%) & $2168(16 \%)$ \\
\hline $1-2$ & $83320(75 \%)$ & $6361(75 \%)$ & $16895(76 \%)$ & $7680(73 \%)$ & $42400(75 \%)$ & $9984(73 \%)$ \\
\hline$\geq 3$ & 9564 (8.6\%) & $425(5.0 \%)$ & $1607(7.2 \%)$ & 756 (7.2\%) & $5306(9.4 \%)$ & $1470(11 \%)$ \\
\hline Oxygen saturation < 95\%, №. (\%) $[\mathrm{n}=261862(83 \%)]$ & $181336(69 \%)$ & $15430(67 \%)$ & $37291(68 \%)$ & $13928(64 \%)$ & $97090(71 \%)$ & $17597(67 \%)$ \\
\hline Dyspnoea, No. (\%) $[n=280719(89 \%)]$ & $224655(80 \%)$ & $21220(84 \%)$ & $51329(83 \%)$ & $17532(78 \%)$ & $112723(78 \%)$ & $21851(79 \%)$ \\
\hline Respiratory distress, No. (\%) [n = 259205 (82\%)] & $179444(69 \%)$ & $18568(78 \%)$ & $37211(70 \%)$ & $14538(67 \%)$ & $91374(68 \%)$ & $17753(68 \%)$ \\
\hline SARI criteria, No. (\%) $[n=260790(83 \%)]$ & $159444(61 \%)$ & $18556(76 \%)$ & $35926(66 \%)$ & $11950(56 \%)$ & $79075(59 \%)$ & 13937 (54\%) \\
\hline SARI without fever criteria, No. (\%) [n = $275676(88 \%)]$ & $211673(77 \%)$ & $21121(84 \%)$ & $46362(78 \%)$ & $15986(72 \%)$ & $108369(76 \%)$ & 19835 (73\%) \\
\hline Hospitalization in state capital, No. (\%) $[n=314615(100 \%)]$ & $162333(52 \%)$ & $13938(51 \%)$ & $45441(64 \%)$ & $15856(66 \%)$ & $78357(48 \%)$ & $8741(30 \%)$ \\
\hline
\end{tabular}

The numbers and proportions within brackets refer to the available data for each variable.

SD - Standard deviation; SARI - Severe acute respiratory infection

a Race was collected as self-reported race or skin colour, originally classified as White (Branco), Black (Preto), Brown (Pardo), Asian (Amarelo), and Indigenous (Indígena)

b Number of chronic comorbidities is the sum of the following comorbidities: cardiovascular, diabetes, renal, neurologic, hematologic, hepatic, chronic respiratory disorder, obesity,

immunosuppression. 
Table A12.25 - Times of the disease, intensive care admissions and need of respiratory support among patients with a defined hospital outcome (Sensitivity Analysis: patients with laboratorial and clinical diagnosis of COVID-19)

\begin{tabular}{|c|c|c|c|c|c|c|}
\hline & $\begin{array}{c}\text { Brazil } \\
(n=284,747)\end{array}$ & $\begin{array}{c}\text { North } \\
(n=25061)\end{array}$ & $\begin{array}{l}\text { Northeast } \\
(n=61322)\end{array}$ & $\begin{array}{c}\text { Central-West } \\
(n=21186)\end{array}$ & $\begin{array}{l}\text { Southeast } \\
(n=149384)\end{array}$ & $\begin{array}{c}\text { South } \\
(n=27794)\end{array}$ \\
\hline \multicolumn{7}{|l|}{ ICU, No. (\%) } \\
\hline $\begin{array}{l}\text { ICU admission [n } \\
=251620(88 \%)]\end{array}$ & $94948(38 \%)$ & $6118(28 \%)$ & $19495(41 \%)$ & $7701(39 \%)$ & $51663(38 \%)$ & $9971(37 \%)$ \\
\hline \multicolumn{7}{|c|}{ Respiratory support, No. (\%) [n=240084; 84\%] } \\
\hline No support & $65310(27 \%)$ & $5621(28 \%)$ & $11301(25 \%)$ & $5040(27 \%)$ & $36362(28 \%)$ & $6986(27 \%)$ \\
\hline Yes, non-invasive & $119717(50 \%)$ & $9859(48 \%)$ & $20958(46 \%)$ & $9470(50 \%)$ & $66356(52 \%)$ & $\begin{array}{l}13074 \\
(50 \%)\end{array}$ \\
\hline \multicolumn{7}{|c|}{ Place of non-invasive respiratory support [n = $113543(95 \%)]^{a}$} \\
\hline In ICU & $32005(28 \%)$ & $1375(14 \%)$ & $5113(26 \%)$ & $2589(29 \%)$ & $19293(31 \%)$ & $3635(28 \%)$ \\
\hline Outside ICU & $81538(72 \%)$ & $8153(86 \%)$ & $14386(74 \%)$ & $6480(71 \%)$ & $43286(69 \%)$ & $9233(72 \%)$ \\
\hline Yes, invasive & $55057(23 \%)$ & $4950(24 \%)$ & $13766(30 \%)$ & $4430(23 \%)$ & $25967(20 \%)$ & $5944(23 \%)$ \\
\hline \multicolumn{7}{|c|}{ Place of invasive respiratory support, $N(\%)[n=53591,97 \%]^{a}$} \\
\hline In ICU & $45997(86 \%)$ & $3963(82 \%)$ & $11135(84 \%)$ & $3550(82 \%)$ & $21912(87 \%)$ & $5437(92 \%)$ \\
\hline Outside ICU & 7594 (14\%) & $895(18 \%)$ & 2127 (16\%) & $796(18 \%)$ & 3297 (13\%) & $479(8.1 \%)$ \\
\hline \multicolumn{7}{|l|}{ Hospitalisation } \\
\hline $\begin{array}{l}\text { Hospital mortality, } \\
\text { No. (\%) } \\
{[n=284747} \\
(100 \%) ; 100 \%)]\end{array}$ & 108566 (38\%) & 11099 (44\%) & 28929 (47\%) & $7278(34 \%)$ & 52777 (35\%) & $8483(31 \%)$ \\
\hline \multicolumn{7}{|l|}{ Length-of-Stay } \\
\hline $\begin{array}{l}\text { Hospital length-of- } \\
\text { stay, median } \\
\text { (IQR) [n=267418; } \\
94 \%]\end{array}$ & $8(4,14)$ & $7(3,14)$ & $8(4,15)$ & $8(4,14)$ & $8(4,14)$ & $8(4,14)$ \\
\hline $\begin{array}{l}\text { ICU LOS, median } \\
\text { (IQR) [n=51777 } \\
(55 \%)]\end{array}$ & $7(3,14)$ & $6(3,12)$ & $6(3,13)$ & $7(3,13)$ & $7(3,14)$ & $9(4,17)$ \\
\hline \multicolumn{7}{|l|}{$\begin{array}{l}\text { Time from onset } \\
\text { of symptoms, } \\
\text { median (IQR) }\end{array}$} \\
\hline $\begin{array}{l}\text { to hospital } \\
\text { admission [n = } \\
248829(87 \%)]\end{array}$ & $7(4,10)$ & $7(5,11)$ & $7(4,10)$ & $7(4,10)$ & $6(4,9)$ & $6(4,9)$ \\
\hline $\begin{array}{l}\text { to ICU admission } \\
\text { [n = } 85714(90 \%)]\end{array}$ & $7(4,10)$ & $9(5,13)$ & $7(4,11)$ & $7(5,11)$ & $7(4,10)$ & $7(4,10)$ \\
\hline $\begin{array}{l}\text { to death }[n= \\
106727(98 \%)]\end{array}$ & $15(9,23)$ & $14(8,21)$ & $14(8,22)$ & $16(10,25)$ & $15(9,23)$ & $17(10,25)$ \\
\hline $\begin{array}{l}\text { Time from hospital } \\
\text { admission to } \\
\text { death }[\mathrm{n}=99041 \\
(91 \%)]\end{array}$ & $9(4,17)$ & $7(3,13)$ & $8(4,16)$ & $10(5,18)$ & $10(5,17)$ & $11(6,20)$ \\
\hline
\end{tabular}

The numbers and proportions in brackets refer to the available data for each variable.

ICU - intensive care unit

a The sum of non-invasive and invasive respiratory support when stratified by place - in ICU and outside ICU - does not match the total respiratory support type because of missing values on the variable ICU admission. 
Table A12.26 - In-hospital mortality stratified by age and sex in Brazil (Sensitivity Analysis: patients with laboratorial and clinical diagnosis of COVID-19)

\begin{tabular}{lcc} 
& Total & By age \\
\hline Total & 284747 & $108566 / 284747(38 \%)$ \\
Age groups & & \\
$20-39$ & 37557 & $4726 / 37557(13 \%)$ \\
$40-49$ & 40980 & $7650 / 40980(19 \%)$ \\
$50-59$ & 52599 & $14590 / 52599(28 \%)$ \\
$60-69$ & 59222 & $25123 / 59222(42 \%)$ \\
$70-79$ & 51503 & $28166 / 51503(55 \%)$ \\
$80+$ & 42886 & $28311 / 42886(66 \%)$ \\
\hline
\end{tabular}

\begin{tabular}{lccc} 
& Total & Female & Male \\
\hline Total & 284697 & $45418 / 123502(37 \%)$ & $63134 / 161195(39 \%)$ \\
Age groups & & & \\
$20-39$ & 37545 & $1977 / 17242(11 \%)$ & $2748 / 20303(14 \%)$ \\
$40-49$ & 40971 & $2764 / 15541(18 \%)$ & $4886 / 25430(19 \%)$ \\
$50-59$ & 52591 & $5361 / 20788(26 \%)$ & $9227 / 31803(29 \%)$ \\
$60-69$ & 59208 & $9699 / 24736(39 \%)$ & $15417 / 34472(45 \%)$ \\
$70-79$ & 51499 & $11569 / 22826(51 \%)$ & $16595 / 28673(58 \%)$ \\
$80+$ & 42883 & $14048 / 22369(63 \%)$ & $14261 / 20514(70 \%)$ \\
\hline
\end{tabular}


Table A12.27 - In-hospital mortality stratified by chronic comorbidities, level of education, self-reported race, and age (Sensitivity Analysis: patients with laboratorial and clinical diagnosis of COVID-19)

\section{Comorbidities}

\begin{tabular}{lcccc} 
& Total & No Comorbidity & $\mathbf{1 - 2}$ Comorbidities & $\geq 3$ Comorbidities \\
\hline Total & 102788 & $5649 / 17154(33 \%)$ & $33113 / 76635(43 \%)$ & $5372 / 8999(60 \%)$ \\
Age groups & & & & \\
$20-39$ & 8252 & $381 / 2815(14 \%)$ & $1125 / 5134(22 \%)$ & $128 / 303(42 \%)$ \\
$40-49$ & 11452 & $483 / 2587(19 \%)$ & $1978 / 8226(24 \%)$ & $280 / 639(44 \%)$ \\
$50-59$ & 18669 & $780 / 3278(24 \%)$ & $4206 / 13915(30 \%)$ & $687 / 1476(47 \%)$ \\
$60-69$ & 24193 & $1193 / 3334(36 \%)$ & $7757 / 18473(42 \%)$ & $1360 / 2386(57 \%)$ \\
$70-79$ & 22021 & $1354 / 2762(49 \%)$ & $9069 / 16953(53 \%)$ & $1524 / 2306(66 \%)$ \\
$80+$ & 18201 & $1458 / 2378(61 \%)$ & $8978 / 13934(64 \%)$ & $1393 / 1889(74 \%)$ \\
\hline
\end{tabular}

\section{Self-reported race *}

\begin{tabular}{|c|c|c|c|c|c|}
\hline & Total & White & Black/Brown & Asian & Indigenous \\
\hline Total & 208812 & $35218 / 96976(36 \%)$ & 45520/107793 (42\%) & $1260 / 3149(40 \%)$ & $392 / 894(44 \%)$ \\
\hline \multicolumn{6}{|c|}{ Age groups } \\
\hline $20-39$ & 27256 & $1201 / 12024(10 \%)$ & $2346 / 14733(16 \%)$ & 49/370 (13\%) & $21 / 129(16 \%)$ \\
\hline $40-49$ & 29353 & $2040 / 13127$ (16\%) & $3662 / 15724$ (23\%) & $74 / 374(20 \%)$ & $29 / 128(23 \%)$ \\
\hline $50-59$ & 38190 & $4231 / 17638$ (24\%) & 6603/19868 (33\%) & $146 / 523(28 \%)$ & $60 / 161(37 \%)$ \\
\hline $60-69$ & 43752 & 7878/20159 (39\%) & 10910/22742 (48\%) & $277 / 682(41 \%)$ & 75/169 (44\%) \\
\hline $70-79$ & 38537 & 9333/17944 (52\%) & $11751 / 19793$ (59\%) & $343 / 656$ (52\%) & $89 / 144(62 \%)$ \\
\hline $80+$ & 31724 & $10535 / 16084(65 \%)$ & $10248 / 14933(69 \%)$ & $371 / 544(68 \%)$ & $118 / 163(72 \%)$ \\
\hline \multirow{2}{*}{\multicolumn{6}{|c|}{$\begin{array}{l}\text { * Race was collected as self-reported race or skin colour, originally classified as White (Branco), Black (Preto), Brown (Pardo), } \\
\text { Asian (Amarelo), and Indigenous (Indígena) }\end{array}$}} \\
\hline & & & & & \\
\hline & Total & Illiterate & Up to high school & High school & College/University \\
\hline Total & 100107 & $4558 / 7397(62 \%)$ & $21069 / 45781$ (46\%) & $9613 / 31949$ (30\%) & $3569 / 14980$ (24\%) \\
\hline \multicolumn{6}{|c|}{ Age groups } \\
\hline $20-39$ & 14770 & $63 / 182(35 \%)$ & $580 / 3003(19 \%)$ & $929 / 7779(12 \%)$ & 285/3806 (7\%) \\
\hline $40-49$ & 15159 & 109/278 (39\%) & $1141 / 4698(24 \%)$ & 1239/6924 (18\%) & $402 / 3259(12 \%)$ \\
\hline $50-59$ & 18903 & $256 / 590(43 \%)$ & $2690 / 8524(32 \%)$ & $1875 / 6808(28 \%)$ & $588 / 2981(20 \%)$ \\
\hline $60-69$ & 20415 & 727/1306 (56\%) & $5228 / 11380(46 \%)$ & $2300 / 5222(44 \%)$ & $867 / 2507$ (35\%) \\
\hline $70-79$ & 17128 & 1367/2204 (62\%) & $5963 / 10339$ (58\%) & $1805 / 3105$ (58\%) & $755 / 1480(51 \%)$ \\
\hline $80+$ & 13732 & 2036/2837 (72\%) & $5467 / 7837(70 \%)$ & $1465 / 2111(69 \%)$ & $672 / 947(71 \%)$ \\
\hline
\end{tabular}


Table A12.28 - In-hospital mortality stratified by ICU admission, respiratory support and age (Sensitivity Analysis: patients with laboratorial and clinical diagnosis of COVID-19)

\begin{tabular}{lccc} 
& Total & No ICU admission & ICU admission \\
\hline Total & 251620 & $37515 / 156672(24 \%)$ & $57175 / 94948(60 \%)$ \\
Age groups & & & \\
$20-39$ & 33297 & $1414 / 24466(6 \%)$ & $2732 / 8831(31 \%)$ \\
$40-49$ & 36490 & $2456 / 25452(10 \%)$ & $4262 / 11038(39 \%)$ \\
$50-59$ & 46899 & $4663 / 30397(15 \%)$ & $8159 / 16502(49 \%)$ \\
$60-69$ & 52261 & $8102 / 30703(26 \%)$ & $13804 / 21558(64 \%)$ \\
$70-79$ & 45196 & $9591 / 24855(39 \%)$ & $14945 / 20341(73 \%)$ \\
$80+$ & 37477 & $11289 / 20799(54 \%)$ & $13273 / 16678(80 \%)$ \\
\hline
\end{tabular}

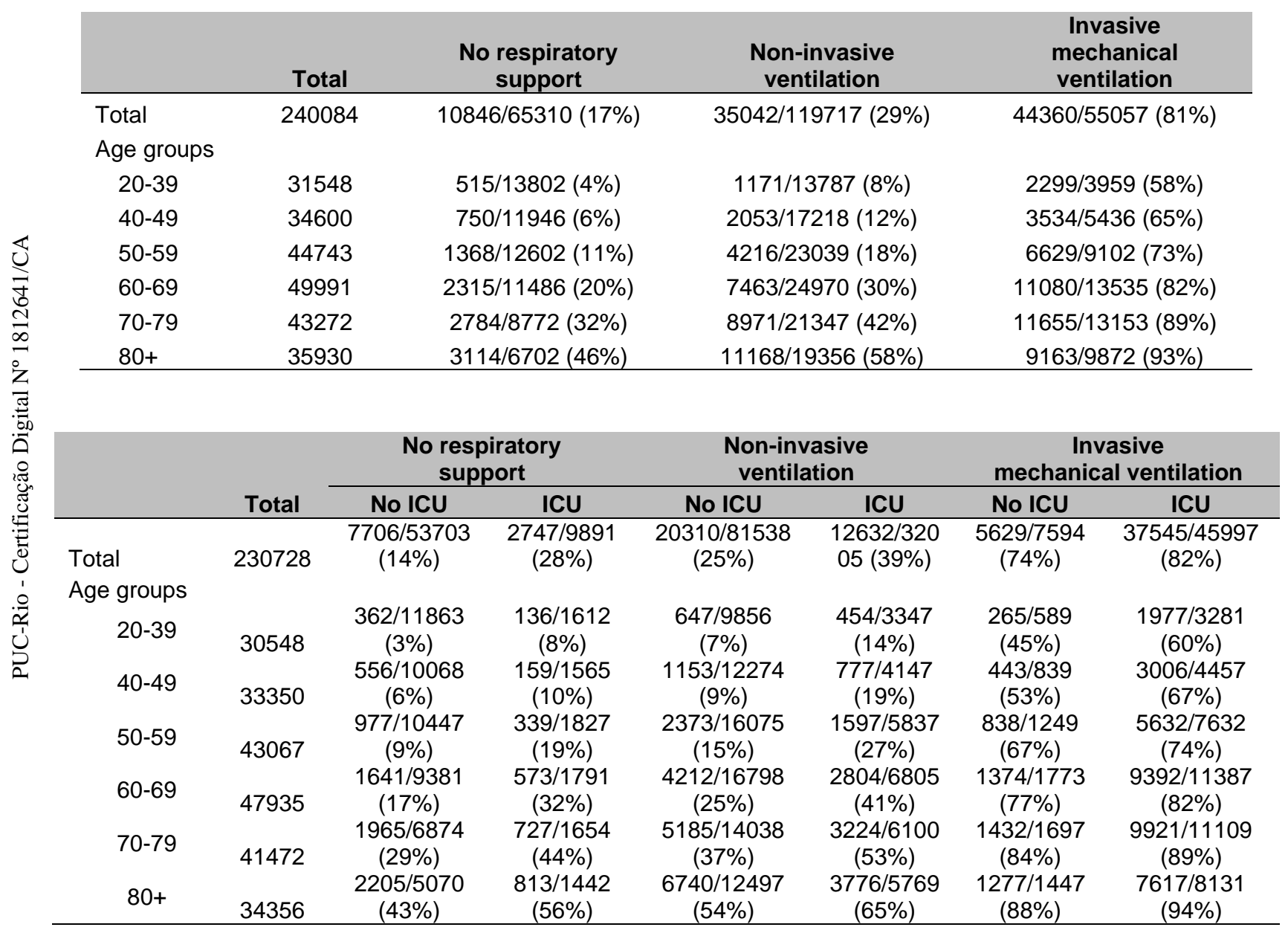



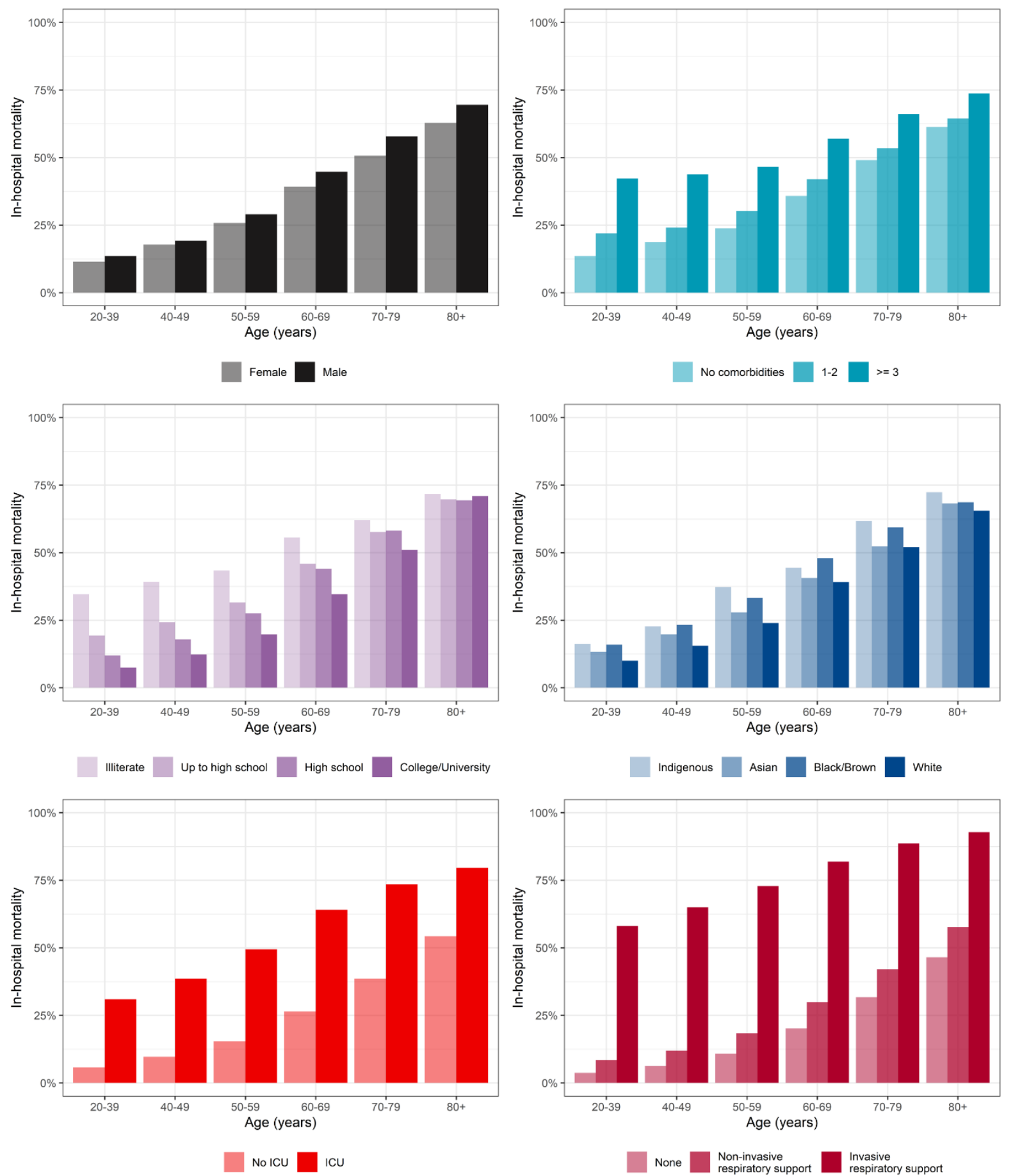

Figure A12.7 - In-hospital mortality stratified by age, sex, comorbidities, level of education, selfreported race *, intensive care admission and invasive mechanical ventilation for hospitalized COVID19 patients in Brazil (Sensitivity Analysis: patients with laboratorial and clinical diagnosis of COVID19)

* Race was collected as self-reported race or skin colour, originally classified as White (Branco), Black (Preto), Brown (Pardo), Asian (Amarelo), and Indigenous (Indígena) 

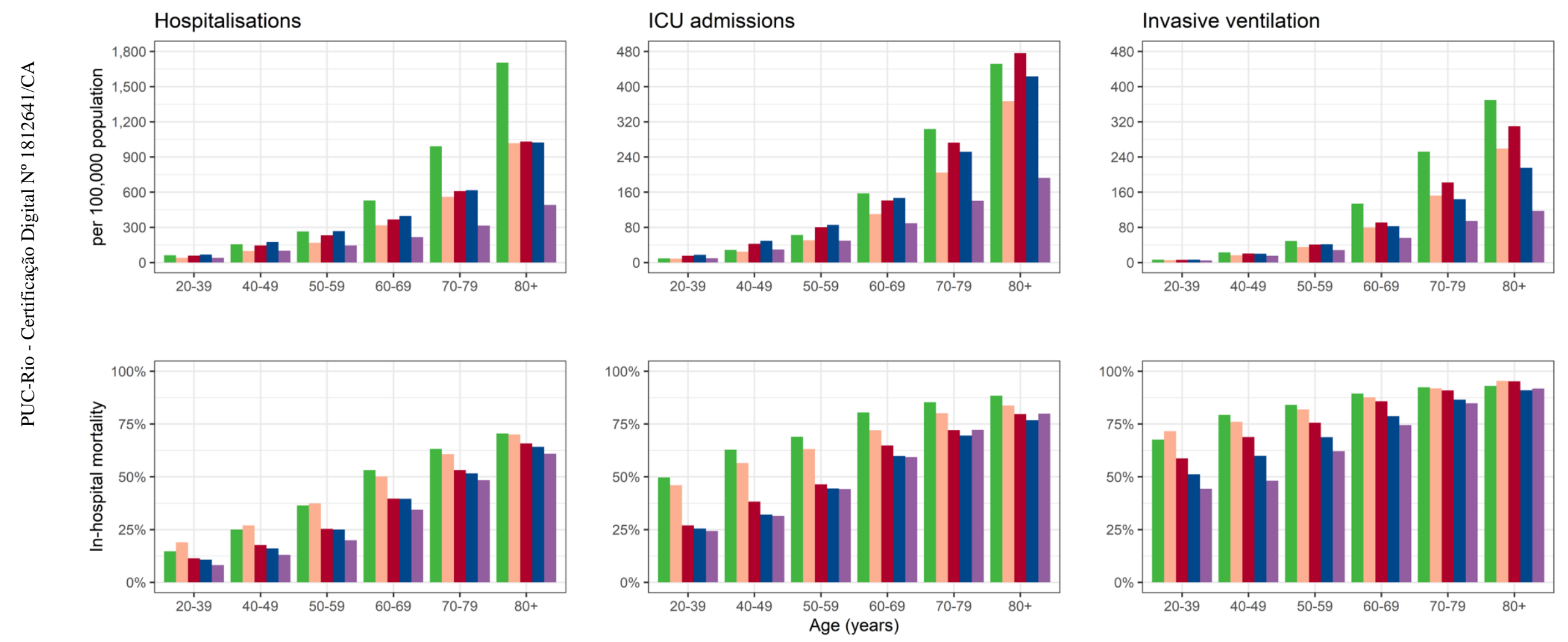

North $\square$ Northeast $\square$ Central-West $\square$ Southeast $\square$ South

Figure A12.8 - Health system burden and in-hospital mortality stratified by age in hospitalised COVID-19 patients in the five regions of Brazil (Sensitivity Analysis: patients with laboratorial and clinical diagnosis of COVID-19) 
Table A12.29 - Health system burden in Brazil and its regions (number / per 100,000 inhabitants) (Sensitivity Analysis: patients with laboratorial and clinical diagnosis of COVID-19)

Hospitalisations per population

\begin{tabular}{|c|c|c|c|c|c|c|c|c|c|c|c|c|}
\hline & \multicolumn{2}{|l|}{ Brazil } & \multicolumn{2}{|l|}{ North } & \multicolumn{2}{|l|}{ Northeast } & \multicolumn{2}{|c|}{ Central-West } & \multicolumn{2}{|l|}{ Southeast } & \multicolumn{2}{|l|}{ South } \\
\hline Total & $284747 / 151778729$ & 187.6 & $25061 / 12049813$ & 208.0 & $61322 / 39882347$ & 153.8 & $21186 / 11678574$ & 181.4 & $149384 / 65803414$ & 227.0 & $27794 / 22364581$ & 124.3 \\
\hline \multicolumn{13}{|l|}{ Age groups } \\
\hline $20-39$ & $37557 / 68451093$ & 54.9 & $3976 / 6448447$ & 61.7 & $7596 / 19048242$ & 39.9 & $3156 / 5484644$ & 57.5 & $19060 / 28059711$ & 67.9 & $3769 / 9410049$ & 40.1 \\
\hline $40-49$ & $40980 / 29255478$ & 140.1 & $3662 / 2357103$ & 155.4 & $7516 / 7654000$ & 98.2 & $3483 / 2386731$ & 145.9 & $22137 / 12717264$ & 174.1 & $4182 / 4140380$ & 101.0 \\
\hline $50-59$ & $52599 / 23875081$ & 220.3 & $4226 / 1600270$ & 264.1 & $10000 / 5930317$ & 168.6 & $4226 / 1825822$ & 231.5 & $28625 / 10724660$ & 266.9 & $5522 / 3794012$ & 145.5 \\
\hline $60-69$ & $59222 / 16732972$ & 353.9 & 5150/974828 & 528.3 & $12343 / 3893805$ & 317.0 & $4241 / 1155857$ & 366.9 & $31455 / 7919342$ & 397.2 & $6033 / 2789140$ & 216.3 \\
\hline $70-79$ & $51503 / 9023052$ & 570.8 & $4657 / 470277$ & 990.3 & $12572 / 2245607$ & 559.8 & $3499 / 575162$ & 608.4 & $26037 / 4225114$ & 616.2 & $4738 / 1506892$ & 314.4 \\
\hline $80+$ & $42886 / 4441053$ & 965.7 & $3390 / 198888$ & 1704.5 & $11295 / 1110376$ & 1017.2 & $2581 / 250358$ & 1030.9 & $22070 / 2157323$ & 1023.0 & $3550 / 724108$ & 490.3 \\
\hline \multicolumn{13}{|c|}{ ICU admissions per population } \\
\hline & \multicolumn{2}{|l|}{ Brazil } & \multicolumn{2}{|l|}{ North } & \multicolumn{2}{|l|}{ Northeast } & \multicolumn{2}{|c|}{ Central-West } & \multicolumn{2}{|l|}{ Southeast } & \multicolumn{2}{|l|}{ South } \\
\hline Total & 94948/151778729 & 62.6 & $6118 / 12049813$ & 50.8 & $19495 / 39882347$ & 48.9 & $7701 / 11678574$ & 65.9 & $51663 / 65803414$ & 78.5 & $9971 / 22364581$ & 44.6 \\
\hline \multicolumn{13}{|l|}{ Age groups } \\
\hline $20-39$ & $8831 / 68451093$ & 12.9 & $590 / 6448447$ & 9.1 & $1661 / 19048242$ & 8.7 & $836 / 5484644$ & 15.2 & $4854 / 28059711$ & 17.3 & $890 / 9410049$ & 9.5 \\
\hline $40-49$ & $11038 / 29255478$ & 37.7 & $672 / 2357103$ & 28.5 & $1881 / 7654000$ & 24.6 & $1010 / 2386731$ & 42.3 & $6265 / 12717264$ & 49.3 & $1210 / 4140380$ & 29.2 \\
\hline $50-59$ & $16502 / 23875081$ & 69.1 & $1001 / 1600270$ & 62.6 & $2999 / 5930317$ & 50.6 & $1468 / 1825822$ & 80.4 & $9154 / 10724660$ & 85.4 & $1880 / 3794012$ & 49.6 \\
\hline $60-69$ & $21558 / 16732972$ & 128.8 & $1531 / 974828$ & 157.1 & $4288 / 3893805$ & 110.1 & $1628 / 1155857$ & 140.8 & $11626 / 7919342$ & 146.8 & $2485 / 2789140$ & 89.1 \\
\hline $70-79$ & $20341 / 9023052$ & 225.4 & $1426 / 470277$ & 303.2 & $4594 / 2245607$ & 204.6 & $1568 / 575162$ & 272.6 & $10640 / 4225114$ & 251.8 & $2113 / 1506892$ & 140.2 \\
\hline $80+$ & $16678 / 4441053$ & 375.5 & $898 / 198888$ & 451.5 & $4072 / 1110376$ & 366.7 & $1191 / 250358$ & 475.7 & $9124 / 2157323$ & 422.9 & 1393/724108 & 192.4 \\
\hline
\end{tabular}

Hospitalisations requiring invasive mechanical ventilation per population

\begin{tabular}{|c|c|c|c|c|c|c|c|c|c|c|c|c|}
\hline & \multicolumn{2}{|l|}{ Brazil } & \multicolumn{2}{|l|}{ North } & \multicolumn{2}{|l|}{ Northeast } & \multicolumn{2}{|c|}{ Central-West } & \multicolumn{2}{|c|}{ Southeast } & \multicolumn{2}{|l|}{ South } \\
\hline Total & $55057 / 151778729$ & 36.3 & $4950 / 12049813$ & 41.1 & $13766 / 39882347$ & 34.5 & $4430 / 11678574$ & 37.9 & $25967 / 65803414$ & 39.5 & $5944 / 22364581$ & 26.6 \\
\hline Age grour & & & & & & & & & & & & \\
\hline $20-39$ & $3959 / 68451093$ & 5.8 & $407 / 6448447$ & 6.3 & $1035 / 19048242$ & 5.4 & $329 / 5484644$ & 6.0 & $1757 / 28059711$ & 6.3 & $431 / 9410049$ & 4.6 \\
\hline $40-49$ & $5436 / 29255478$ & 18.6 & $540 / 2357103$ & 22.9 & $1261 / 7654000$ & 16.5 & $481 / 2386731$ & 20.2 & $2531 / 12717264$ & 19.9 & $623 / 4140380$ & 15.0 \\
\hline $50-59$ & $9102 / 23875081$ & 38.1 & $779 / 1600270$ & 48.7 & $2071 / 5930317$ & 34.9 & $745 / 1825822$ & 40.8 & $4446 / 10724660$ & 41.5 & $1061 / 3794012$ & 28.0 \\
\hline $60-69$ & $13535 / 16732972$ & 80.9 & $1304 / 974828$ & 133.8 & $3105 / 3893805$ & 79.7 & $1053 / 1155857$ & 91.1 & $6512 / 7919342$ & 82.2 & $1561 / 2789140$ & 56.0 \\
\hline $70-79$ & $13153 / 9023052$ & 145.8 & $1186 / 470277$ & 252.2 & $3420 / 2245607$ & 152.3 & $1046 / 575162$ & 181.9 & $6082 / 4225114$ & 143.9 & $1419 / 1506892$ & 94.2 \\
\hline $80+$ & $9872 / 4441053$ & 222.3 & $734 / 198888$ & 369.1 & $2874 / 1110376$ & 258.8 & $776 / 250358$ & 310.0 & $4639 / 2157323$ & 215.0 & $849 / 724108$ & 117.2 \\
\hline
\end{tabular}


Table A12.30 - Overall in-hospital mortality, among those admitted to the ICU or under invasive mechanical ventilation, stratified by age and Brazilian regions (Sensitivity Analysis: patients with laboratorial and clinical diagnosis of COVID-19)

\section{In-hospital mortality (Overall)}

\begin{tabular}{|c|c|c|c|c|c|c|}
\hline & Brazil & North & Northeast & Central-West & Southeast & South \\
\hline Total & $108566 / 284747(38.1 \%)$ & $11099 / 25061(44.3 \%)$ & $28929 / 61322(47.2 \%)$ & 7278/21186 (34.4\%) & $52777 / 149384(35.3 \%)$ & $8483 / 27794(30.5 \%)$ \\
\hline \multicolumn{7}{|l|}{ Age groups } \\
\hline $20-39$ & $4726 / 37557$ (12.6\%) & $584 / 3976(14.7 \%)$ & 1439/7596 (18.9\%) & $358 / 3156(11.3 \%)$ & $2038 / 19060$ (10.7\%) & $307 / 3769(8.1 \%)$ \\
\hline $40-49$ & $7650 / 40980(18.7 \%)$ & $914 / 3662(25 \%)$ & $2026 / 7516(27 \%)$ & $615 / 3483(17.7 \%)$ & $3553 / 22137(16.1 \%)$ & $542 / 4182(13 \%)$ \\
\hline $50-59$ & $14590 / 52599(27.7 \%)$ & $1537 / 4226(36.4 \%)$ & $3740 / 10000(37.4 \%)$ & $1069 / 4226(25.3 \%)$ & $7143 / 28625(25 \%)$ & $1101 / 5522(19.9 \%)$ \\
\hline $60-69$ & $25123 / 59222(42.4 \%)$ & $2733 / 5150(53.1 \%)$ & $6183 / 12343(50.1 \%)$ & $1681 / 4241(39.6 \%)$ & $12449 / 31455$ (39.6\%) & $2077 / 6033(34.4 \%)$ \\
\hline $70-79$ & $28166 / 51503(54.7 \%)$ & $2942 / 4657(63.2 \%)$ & $7634 / 12572(60.7 \%)$ & $1858 / 3499(53.1 \%)$ & $13438 / 26037(51.6 \%)$ & $2294 / 4738(48.4 \%)$ \\
\hline $80+$ & $28311 / 42886(66 \%)$ & $2389 / 3390(70.5 \%)$ & $7907 / 11295(70 \%)$ & $1697 / 2581(65.7 \%)$ & $14156 / 22070(64.1 \%)$ & $2162 / 3550(60.9 \%)$ \\
\hline
\end{tabular}

\section{In-hospital mortality (ICU admissions)}

\begin{tabular}{|c|c|c|c|c|c|c|}
\hline & Brazil & North & Northeast & Central-West & Southeast & $\begin{array}{l}\text { South } \\
\end{array}$ \\
\hline Total & $57175 / 94948(60.2 \%)$ & $4647 / 6118(76 \%)$ & $13899 / 19495$ (71.3\%) & $4427 / 7701(57.5 \%)$ & $28661 / 51663(55.5 \%)$ & $5541 / 9971(55.6 \%)$ \\
\hline \multicolumn{7}{|c|}{ Age groups } \\
\hline $20-39$ & $2732 / 8831(30.9 \%)$ & $293 / 590(49.7 \%)$ & $764 / 1661(46 \%)$ & $225 / 836(26.9 \%)$ & $1234 / 4854(25.4 \%)$ & $216 / 890(24.3 \%)$ \\
\hline $40-49$ & $4262 / 11038(38.6 \%)$ & $422 / 672(62.8 \%)$ & $1063 / 1881(56.5 \%)$ & $386 / 1010(38.2 \%)$ & $2010 / 6265(32.1 \%)$ & $381 / 1210(31.5 \%)$ \\
\hline $50-59$ & $8159 / 16502(49.4 \%)$ & $690 / 1001(68.9 \%)$ & $1893 / 2999(63.1 \%)$ & $681 / 1468(46.4 \%)$ & $4065 / 9154(44.4 \%)$ & $830 / 1880(44.1 \%)$ \\
\hline $60-69$ & $13804 / 21558(64 \%)$ & $1232 / 1531(80.5 \%)$ & $3090 / 4288(72.1 \%)$ & $1055 / 1628(64.8 \%)$ & $6952 / 11626(59.8 \%)$ & $1475 / 2485(59.4 \%)$ \\
\hline $70-79$ & $14945 / 20341(73.5 \%)$ & $1216 / 1426(85.3 \%)$ & $3678 / 4594(80.1 \%)$ & $1131 / 1568(72.1 \%)$ & $7394 / 10640(69.5 \%)$ & $1526 / 2113(72.2 \%)$ \\
\hline $80+$ & $13273 / 16678(79.6 \%)$ & $794 / 898(88.4 \%)$ & $3411 / 4072(83.8 \%)$ & $949 / 1191(79.7 \%)$ & $7006 / 9124(76.8 \%)$ & $1113 / 1393(79.9 \%)$ \\
\hline
\end{tabular}

\section{In-hospital mortality (Invasive mechanical ventilation)}

\begin{tabular}{|c|c|c|c|c|c|c|}
\hline & Brazil & North & Northeast & Central-West & Southeast & South \\
\hline Total & $44360 / 55057(80.6 \%)$ & $4303 / 4950(86.9 \%)$ & $12004 / 13766(87.2 \%)$ & $3680 / 4430(83.1 \%)$ & $20077 / 25967$ (77.3\%) & $4296 / 5944(72.3 \%)$ \\
\hline \multicolumn{7}{|c|}{ Age groups } \\
\hline $20-39$ & $2299 / 3959(58.1 \%)$ & $275 / 407(67.6 \%)$ & $741 / 1035(71.6 \%)$ & $193 / 329(58.7 \%)$ & $899 / 1757(51.2 \%)$ & $191 / 431(44.3 \%)$ \\
\hline $40-49$ & $3534 / 5436(65 \%)$ & $428 / 540(79.3 \%)$ & $959 / 1261(76.1 \%)$ & $331 / 481(68.8 \%)$ & $1516 / 2531(59.9 \%)$ & $300 / 623(48.2 \%)$ \\
\hline $50-59$ & $6629 / 9102(72.8 \%)$ & $655 / 779(84.1 \%)$ & $1697 / 2071(81.9 \%)$ & $563 / 745(75.6 \%)$ & $3055 / 4446(68.7 \%)$ & $659 / 1061(62.1 \%)$ \\
\hline $60-69$ & $11080 / 13535(81.9 \%)$ & $1166 / 1304(89.4 \%)$ & $2722 / 3105(87.7 \%)$ & $903 / 1053(85.8 \%)$ & $5126 / 6512(78.7 \%)$ & $1163 / 1561(74.5 \%)$ \\
\hline $70-79$ & $11655 / 13153(88.6 \%)$ & $1096 / 1186(92.4 \%)$ & $3142 / 3420(91.9 \%)$ & $951 / 1046(90.9 \%)$ & $5262 / 6082(86.5 \%)$ & $1204 / 1419(84.8 \%)$ \\
\hline $80+$ & $9163 / 9872(92.8 \%)$ & 683/734 (93.1\%) & $2743 / 2874(95.4 \%)$ & $739 / 776(95.2 \%)$ & $4219 / 4639(90.9 \%)$ & $779 / 849(91.8 \%)$ \\
\hline
\end{tabular}


Table A12.31 - Hospitalisations and ICU admissions per hospital and ICU beds in Brazil and regions (Sensitivity Analysis: patients with laboratorial and clinical diagnosis of COVID-19)*

\begin{tabular}{|c|c|c|c|c|c|c|c|c|c|c|c|c|}
\hline & \multicolumn{2}{|c|}{ Brazil } & \multicolumn{2}{|c|}{ North } & \multicolumn{2}{|c|}{ Northeast } & \multicolumn{2}{|c|}{ Central-West } & \multicolumn{2}{|c|}{ Southeast } & \multicolumn{2}{|c|}{ South } \\
\hline & $\mathrm{N}$ & rate & $\mathrm{N}$ & rate & $\mathrm{N}$ & rate & $\mathrm{N}$ & rate & $\mathrm{N}$ & rate & $\mathrm{N}$ & rate \\
\hline $\begin{array}{c}\text { All } \\
\text { hospitalizations } \\
\text { (n/per 100,000 } \\
\text { hospital beds) }\end{array}$ & $\begin{array}{l}28474 / \\
356344\end{array}$ & 79,908 & $\begin{array}{l}2506 / \\
23719\end{array}$ & 105,658 & $\begin{array}{l}61322 / \\
87604\end{array}$ & 69,999 & $\begin{array}{l}21186 / \\
29606\end{array}$ & 71,560 & $\begin{array}{c}149384 / 1 \\
57510\end{array}$ & 94,841 & $\begin{array}{l}18525 / \\
57905\end{array}$ & 31,992 \\
\hline $\begin{array}{l}\text { ICU admissions } \\
\text { (n/per } 1000 \text { ICU } \\
\text { beds) }\end{array}$ & $\begin{array}{l}94948 / \\
37692\end{array}$ & 2,519 & $\begin{array}{c}6118 / \\
1686\end{array}$ & 3,629 & $\begin{array}{c}19495 / \\
7171\end{array}$ & 2,719 & $\begin{array}{l}7701 / \\
3340\end{array}$ & 2,306 & $\begin{array}{l}51663 / \\
20403\end{array}$ & 2,532 & $\begin{array}{l}9971 / \\
5092\end{array}$ & 1,958 \\
\hline
\end{tabular}

\footnotetext{
${ }^{*}$ Beds data on February 2020
} 

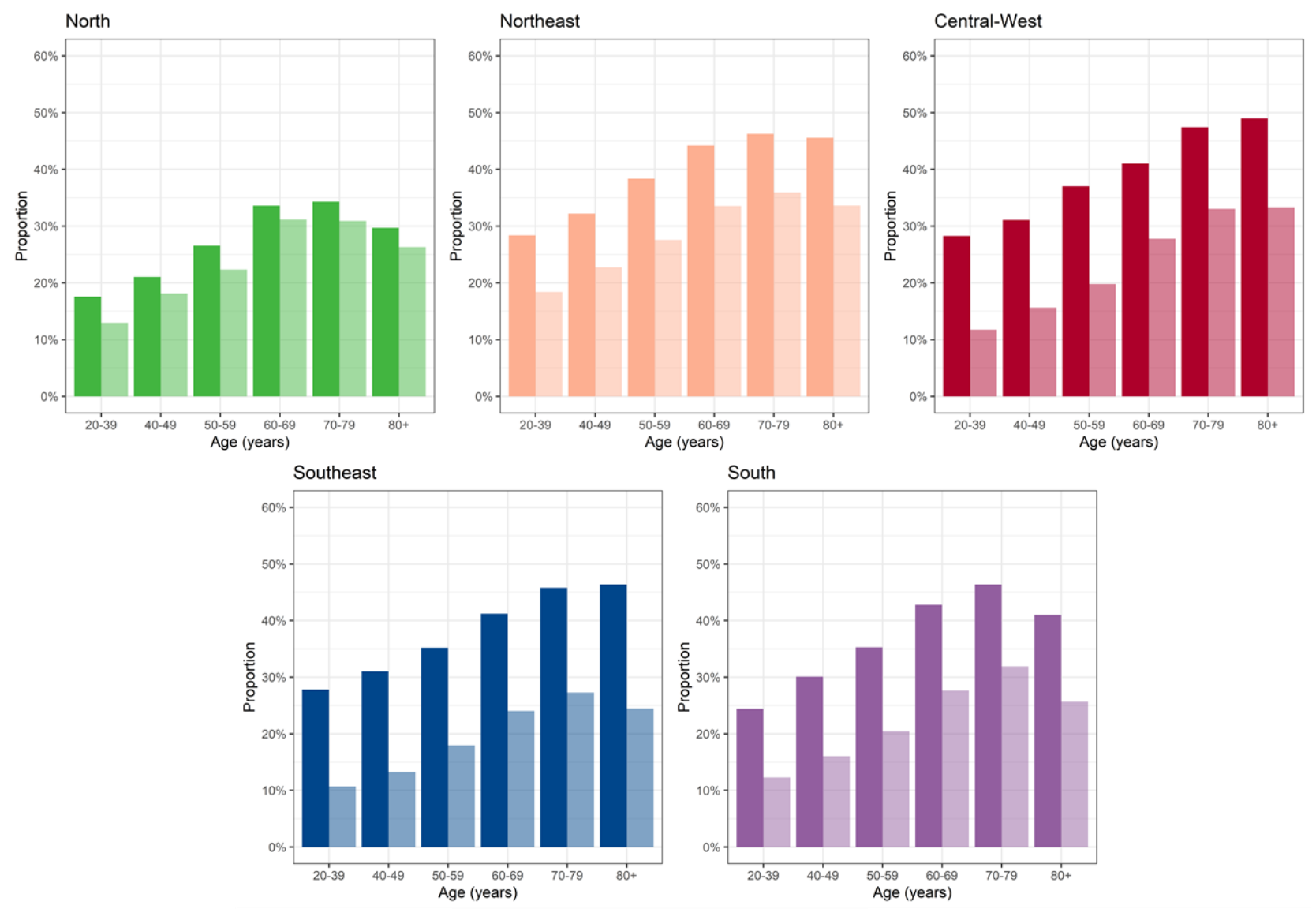

ICU Invasive ventilation

Figure A12.9 - Proportion of intensive care unit admission and use of mechanical ventilation stratified by age in hospitalised COVID-19 patients in the five regions of Brazil (Sensitivity Analysis: patients with laboratorial and clinical diagnosis of COVID-19) 
Table A12.32 - Use of resources in terms of proportions of hospitalised patients admitted to the ICU and under invasive mechanical ventilation stratified by age and region (Sensitivity Analysis: patients with laboratorial and clinical diagnosis of COVID-19)

Proportion of ICU admission

\begin{tabular}{|c|c|c|c|c|c|c|}
\hline & Brazil & North & Northeast & Central-West & Southeast & South \\
\hline Total & $94948 / 251620$ (37.7\%) & 6118/22070 (27.7\%) & $19495 / 48095$ (40.5\%) & 7701/19886 (38.7\%) & $51663 / 134801$ (38.3\%) & $9971 / 26768(37.2 \%)$ \\
\hline \multicolumn{7}{|c|}{ Age groups } \\
\hline $20-39$ & $8831 / 33297$ (26.5\%) & $590 / 3367$ (17.5\%) & $1661 / 5859(28.3 \%)$ & $836 / 2960$ (28.2\%) & $4854 / 17465$ (27.8\%) & $890 / 3646(24.4 \%)$ \\
\hline $40-49$ & $11038 / 36490$ (30.2\%) & 672/3194 (21\%) & $1881 / 5840(32.2 \%)$ & $1010 / 3249$ (31.1\%) & 6265/20185 (31\%) & $1210 / 4022(30.1 \%)$ \\
\hline $50-59$ & $16502 / 46899$ (35.2\%) & $1001 / 3771$ (26.5\%) & 2999/7822 (38.3\%) & $1468 / 3967$ (37\%) & 9154/26007 (35.2\%) & 1880/5332 (35.3\%) \\
\hline $60-69$ & $21558 / 52261$ (41.3\%) & $1531 / 4557(33.6 \%)$ & 4288/9704 (44.2\%) & 1628/3969 (41\%) & 11626/28222 (41.2\%) & $2485 / 5809(42.8 \%)$ \\
\hline $70-79$ & $20341 / 45196$ (45\%) & $1426 / 4156(34.3 \%)$ & $4594 / 9933(46.2 \%)$ & 1568/3308 (47.4\%) & $10640 / 23240$ (45.8\%) & $2113 / 4559(46.3 \%)$ \\
\hline $80+$ & $16678 / 37477$ (44.5\%) & 898/3025 (29.7\%) & $4072 / 8937$ (45.6\%) & $1191 / 2433(49 \%)$ & $9124 / 19682$ (46.4\%) & $1393 / 3400(41 \%)$ \\
\hline
\end{tabular}

Proportion on invasive mechanical ventilation

\begin{tabular}{|c|c|c|c|c|c|c|}
\hline & Brazil & North & Northeast & Central-West & Southeast & South \\
\hline Total & $55057 / 240084(22.9 \%)$ & $4950 / 20430(24.2 \%)$ & $13766 / 46025$ (29.9\%) & $4430 / 18940(23.4 \%)$ & $25967 / 128685$ (20.2\%) & $5944 / 26004(22.9 \%)$ \\
\hline \multicolumn{7}{|c|}{ Age groups } \\
\hline $20-39$ & $3959 / 31548$ (12.5\%) & 407/3142 (13\%) & $1035 / 5627$ (18.4\%) & $329 / 2806(11.7 \%)$ & $1757 / 16456(10.7 \%)$ & $431 / 3517$ (12.3\%) \\
\hline $40-49$ & $5436 / 34600$ (15.7\%) & $540 / 2980(18.1 \%)$ & $1261 / 5546(22.7 \%)$ & $481 / 3079$ (15.6\%) & $2531 / 19105$ (13.2\%) & $623 / 3890$ (16\%) \\
\hline $50-59$ & $9102 / 44743$ (20.3\%) & 779/3491 (22.3\%) & $2071 / 7520(27.5 \%)$ & 745/3766 (19.8\%) & $4446 / 24774$ (17.9\%) & 1061/5192 (20.4\%) \\
\hline $60-69$ & $13535 / 49991(27.1 \%)$ & $1304 / 4186(31.2 \%)$ & $3105 / 9262(33.5 \%)$ & 1053/3792 (27.8\%) & $6512 / 27103$ (24\%) & $1561 / 5648(27.6 \%)$ \\
\hline $70-79$ & $13153 / 43272(30.4 \%)$ & $1186 / 3839$ (30.9\%) & $3420 / 9520$ (35.9\%) & 1046/3168 (33\%) & 6082/22295 (27.3\%) & $1419 / 4450(31.9 \%)$ \\
\hline $80+$ & $9872 / 35930(27.5 \%)$ & 734/2792 (26.3\%) & $2874 / 8550(33.6 \%)$ & 776/2329 (33.3\%) & 4639/18952 (24.5\%) & $849 / 3307$ (25.7\%) \\
\hline
\end{tabular}


Table A 12.33 - In-hospital mortality by comorbidities numbers, ICU admission and respiratory support in main analysis and multiple imputed data in Brazil (sensitivity analysis)

\begin{tabular}{|c|c|c|}
\hline & $\begin{array}{c}\text { Original } \\
\text { (complete cases) }\end{array}$ & Imputed values \\
\hline Number of comorbidities & & \\
\hline 0 & $4494 / 13836(32 \%)$ & $24.6 \%$ \\
\hline $1-2$ & $26933 / 62766(43 \%)$ & $35.9 \%$ \\
\hline$\geq 3$ & $4685 / 7798(60 \%)$ & $48.7 \%$ \\
\hline Respiratory Support & & \\
\hline None & $8655 / 54314(16 \%)$ & $16.4 \%$ \\
\hline Yes, non-invasive & $28287 / 96729(29 \%)$ & $29.7 \%$ \\
\hline Yes, invasive & $36046 / 45205(80 \%)$ & $80.0 \%$ \\
\hline ICU admission & $47002 / 79687(59 \%)$ & $59.4 \%$ \\
\hline
\end{tabular}


Table A 12.34 - In-hospital mortality by comorbidities numbers and age in main analysis and multiple imputed data in Brazil (sensitivity analysis)

\begin{tabular}{|c|c|c|c|c|}
\hline & Total & No Comorbidity & 1-2 Comorbidities & $\geq 3$ Comorbidities \\
\hline \multicolumn{5}{|l|}{ Age groups } \\
\hline $20-39$ & 6780 & $291 / 2245$ (13\%) & $937 / 4278(22 \%)$ & $104 / 257(40 \%)$ \\
\hline $40-49$ & 9547 & $396 / 2171(18 \%)$ & 1591/6824 (23\%) & 243/552 (44\%) \\
\hline $50-59$ & 15438 & $625 / 2691(23 \%)$ & $3432 / 11484(30 \%)$ & $594 / 1263(47 \%)$ \\
\hline $60-69$ & 19886 & $951 / 2691(35 \%)$ & $6300 / 15112(42 \%)$ & $1187 / 2083(57 \%)$ \\
\hline $70-79$ & 17957 & $1051 / 2165(49 \%)$ & $7360 / 13772(53 \%)$ & $1349 / 2020(67 \%)$ \\
\hline $80+$ & 14792 & $1180 / 1873(63 \%)$ & $7313 / 11296(65 \%)$ & $1208 / 1623(74 \%)$ \\
\hline Multiple imputed & & No Comorbidity & 1-2 Comorbidities & $\geq 3$ Comorbidities \\
\hline \multicolumn{5}{|l|}{ Age groups } \\
\hline 20-39 & & $7.9 \%$ & $12.2 \%$ & $19.0 \%$ \\
\hline $40-49$ & & $13.3 \%$ & $16.8 \%$ & $25.9 \%$ \\
\hline $50-59$ & & $20.4 \%$ & $24.9 \%$ & $37.0 \%$ \\
\hline $60-69$ & & $34.5 \%$ & $38.7 \%$ & $52.4 \%$ \\
\hline $70-79$ & & $48.5 \%$ & $51.5 \%$ & $62.9 \%$ \\
\hline $80+$ & & $63 \%$ & $63.9 \%$ & $71.7 \%$ \\
\hline
\end{tabular}


Table A 12.35 -In-hospital mortality by comorbidities numbers, ICU admission and respiratory support in main analysis and multiple imputed data stratified by age (sensitivity analysis)

In-hospital mortality (ICU admissions)

\begin{tabular}{|c|c|c|c|c|c|c|c|c|c|c|}
\hline & \multicolumn{2}{|c|}{ North } & \multicolumn{2}{|c|}{ Northeast } & \multicolumn{2}{|c|}{ Central-West } & \multicolumn{2}{|c|}{ Southeast } & \multicolumn{2}{|c|}{ South } \\
\hline & $\begin{array}{c}\text { Complete } \\
\text { case }\end{array}$ & Imputed & $\begin{array}{c}\text { Complete } \\
\text { case }\end{array}$ & Imputed & $\begin{array}{c}\text { Complete } \\
\text { case }\end{array}$ & Imputed & $\begin{array}{c}\text { Complete } \\
\text { case }\end{array}$ & Imputed & $\begin{array}{c}\text { Complete } \\
\text { case }\end{array}$ & Imputed \\
\hline \multicolumn{11}{|c|}{ Age groups } \\
\hline $\begin{array}{l}20- \\
39\end{array}$ & $\begin{array}{l}195 / 334 \\
(58.4 \%) \\
\end{array}$ & $57.5 \%$ & $\begin{array}{c}579 / 1279 \\
(45.3 \%)\end{array}$ & $42.1 \%$ & $\begin{array}{l}185 / 732 \\
(25.3 \%) \\
\end{array}$ & $25.4 \%$ & $\begin{array}{c}1065 / 4354 \\
(24.5 \%) \\
\end{array}$ & $24.6 \%$ & $\begin{array}{l}201 / 813 \\
(24.7 \%)\end{array}$ & $24.6 \%$ \\
\hline $\begin{array}{l}40- \\
49 \\
\end{array}$ & $\begin{array}{l}265 / 404 \\
(65.6 \%) \\
\end{array}$ & $65.7 \%$ & $\begin{array}{c}799 / 1440 \\
(55.5 \%) \\
\end{array}$ & $52.4 \%$ & $\begin{array}{l}324 / 890 \\
(36.4 \%) \\
\end{array}$ & $36.3 \%$ & $\begin{array}{c}1763 / 5630 \\
(31.3 \%)\end{array}$ & $31.5 \%$ & $\begin{array}{c}352 / 1114 \\
(31.6 \%) \\
\end{array}$ & $31.5 \%$ \\
\hline $\begin{array}{l}50- \\
59 \\
\end{array}$ & $\begin{array}{l}468 / 645 \\
(72.6 \%) \\
\end{array}$ & $72.8 \%$ & $\begin{array}{c}1441 / 2324 \\
(62 \%) \\
\end{array}$ & $60.5 \%$ & $\begin{array}{c}554 / 1266 \\
(43.8 \%) \\
\end{array}$ & $44.1 \%$ & $\begin{array}{c}3513 / 8097 \\
(43.4 \%) \\
\end{array}$ & $43.6 \%$ & $\begin{array}{c}756 / 1702 \\
(44.4 \%) \\
\end{array}$ & $44.3 \%$ \\
\hline $\begin{array}{l}60- \\
69 \\
\end{array}$ & $\begin{array}{l}800 / 950 \\
(84.2 \%) \\
\end{array}$ & $84.1 \%$ & $\begin{array}{c}2350 / 3318 \\
(70.8 \%)\end{array}$ & $70.8 \%$ & $\begin{array}{c}896 / 1420 \\
(63.1 \%)\end{array}$ & $62.7 \%$ & $\begin{array}{c}5980 / 10094 \\
(59.2 \%)\end{array}$ & $59.5 \%$ & $\begin{array}{c}1346 / 2276 \\
(59.1 \%) \\
\end{array}$ & $59.1 \%$ \\
\hline $\begin{array}{l}70- \\
79 \\
\end{array}$ & $\begin{array}{l}779 / 891 \\
(87.4 \%) \\
\end{array}$ & $87.7 \%$ & $\begin{array}{c}2720 / 3401 \\
(80 \%)\end{array}$ & $79.4 \%$ & $\begin{array}{c}954 / 1345 \\
(70.9 \%)\end{array}$ & $70.9 \%$ & $\begin{array}{c}6408 / 9264 \\
(69.2 \%)\end{array}$ & $69.4 \%$ & $\begin{array}{c}1396 / 1947 \\
(71.7 \%)\end{array}$ & $71.6 \%$ \\
\hline $80+$ & $\begin{array}{l}515 / 562 \\
(91.6 \%) \\
\end{array}$ & $91.7 \%$ & $\begin{array}{c}2594 / 3105 \\
(83.5 \%)\end{array}$ & $83.9 \%$ & $\begin{array}{c}821 / 1029 \\
(79.8 \%)\end{array}$ & $79.9 \%$ & $\begin{array}{c}5964 / 7785 \\
(76.6 \%)\end{array}$ & $77.0 \%$ & $\begin{array}{c}1019 / 1276 \\
(79.9 \%)\end{array}$ & $79.7 \%$ \\
\hline
\end{tabular}

In-hospital mortality (Invasive mechanical ventilation)

\begin{tabular}{|c|c|c|c|c|c|c|c|c|c|c|}
\hline & \multicolumn{2}{|c|}{ North } & \multicolumn{2}{|c|}{ Northeast } & \multicolumn{2}{|c|}{ Central-West } & \multicolumn{2}{|c|}{ Southeast } & \multicolumn{2}{|c|}{ South } \\
\hline & $\begin{array}{c}\text { Complete } \\
\text { case }\end{array}$ & Imputed & $\begin{array}{c}\text { Complete } \\
\text { case }\end{array}$ & Imputed & $\begin{array}{c}\text { Complete } \\
\text { case }\end{array}$ & Imputed & $\begin{array}{c}\text { Complete } \\
\text { case }\end{array}$ & Imputed & $\begin{array}{c}\text { Complete } \\
\text { case }\end{array}$ & Imputed \\
\hline Age & roups & & & & & & & & & \\
\hline $\begin{array}{l}20- \\
39 \\
\end{array}$ & $\begin{array}{l}193 / 250 \\
(77.2 \%) \\
\end{array}$ & $75.7 \%$ & $\begin{array}{l}551 / 791 \\
(69.7 \%) \\
\end{array}$ & $65.4 \%$ & $\begin{array}{l}164 / 279 \\
(58.8 \%)\end{array}$ & $57.4 \%$ & $\begin{array}{c}774 / 1559 \\
(49.6 \%)\end{array}$ & $49.8 \%$ & $\begin{array}{l}176 / 399 \\
(44.1 \%) \\
\end{array}$ & $43.8 \%$ \\
\hline $\begin{array}{l}40- \\
49 \\
\end{array}$ & $\begin{array}{l}269 / 334 \\
(80.5 \%) \\
\end{array}$ & $80.2 \%$ & $\begin{array}{l}717 / 947 \\
(75.7 \%) \\
\end{array}$ & $72.5 \%$ & $\begin{array}{l}273 / 397 \\
(68.8 \%) \\
\end{array}$ & $67.8 \%$ & $\begin{array}{c}1317 / 2239 \\
(58.8 \%) \\
\end{array}$ & $59 \%$ & $\begin{array}{c}274 / 571 \\
(48 \%) \\
\end{array}$ & $48 \%$ \\
\hline $\begin{array}{l}50- \\
59 \\
\end{array}$ & $\begin{array}{l}446 / 520 \\
(85.8 \%)\end{array}$ & $85.8 \%$ & $\begin{array}{c}1291 / 1579 \\
(81.8 \%) \\
\end{array}$ & $79.9 \%$ & $\begin{array}{l}456 / 605 \\
(75.4 \%) \\
\end{array}$ & $74.4 \%$ & $\begin{array}{c}2662 / 3923 \\
(67.9 \%) \\
\end{array}$ & $68.1 \%$ & $\begin{array}{c}604 / 959 \\
(63 \%) \\
\end{array}$ & $62.6 \%$ \\
\hline $\begin{array}{l}60- \\
69\end{array}$ & $\begin{array}{l}748 / 821 \\
(91.1 \%)\end{array}$ & $91.1 \%$ & $\begin{array}{c}2056 / 2353 \\
(87.4 \%)\end{array}$ & $86.4 \%$ & $\begin{array}{l}743 / 875 \\
(84.9 \%)\end{array}$ & $84.5 \%$ & $\begin{array}{c}4424 / 5673 \\
(78 \%)\end{array}$ & $78.6 \%$ & $\begin{array}{c}1057 / 1426 \\
(74.1 \%)\end{array}$ & $74.2 \%$ \\
\hline $\begin{array}{l}70- \\
79 \\
\end{array}$ & $\begin{array}{l}711 / 765 \\
(92.9 \%) \\
\end{array}$ & $93.4 \%$ & $\begin{array}{c}2289 / 2494 \\
(91.8 \%) \\
\end{array}$ & $91.3 \%$ & $\begin{array}{l}787 / 863 \\
(91.2 \%) \\
\end{array}$ & $91 \%$ & $\begin{array}{c}4551 / 5291 \\
(86 \%) \\
\end{array}$ & $86.5 \%$ & $\begin{array}{c}1095 / 1293 \\
(84.7 \%) \\
\end{array}$ & $84.6 \%$ \\
\hline $80+$ & $\begin{array}{l}443 / 465 \\
(95.3 \%)\end{array}$ & $95.3 \%$ & $\begin{array}{c}2059 / 2158 \\
(95.4 \%)\end{array}$ & $95.1 \%$ & $\begin{array}{l}616 / 648 \\
(95.1 \%)\end{array}$ & $94.9 \%$ & $\begin{array}{c}3597 / 3963 \\
(90.8 \%)\end{array}$ & $91.2 \%$ & $\begin{array}{l}703 / 765 \\
(91.9 \%)\end{array}$ & $91.8 \%$ \\
\hline
\end{tabular}


12.3.5

Appendix A4.5 - Comparison of multicenter COVID-19 cohorts

\begin{tabular}{|c|c|c|c|c|c|c|}
\hline & \multicolumn{6}{|c|}{ Multicentre study cohort } \\
\hline Authors & Ranzani et al. & $\begin{array}{l}\text { (DOCHERTY } \\
\text { et al., 2020) }\end{array}$ & $\begin{array}{l}\text { (GRASSELLI } \\
\text { et al., 2020) }\end{array}$ & $\begin{array}{l}\text { (GUPTA et } \\
\text { al., 2020) }\end{array}$ & $\begin{array}{c}\text { (KARAGIANNIDIS } \\
\text { et al., 2020) }\end{array}$ & $\begin{array}{l}\text { (ÑAMENDYS- } \\
\text { SILVA; } \\
\text { GUTIÉRREZ- } \\
\text { VILLASEÑOR; } \\
\text { ROMERO- } \\
\text { GONZÁLEZ, } \\
2020 \text { ) }\end{array}$ \\
\hline Country & Brazil & $\begin{array}{c}\text { United } \\
\text { Kingdom }\end{array}$ & Italy & $\begin{array}{l}\text { United } \\
\text { States }\end{array}$ & Germany & Mexico \\
\hline Coverage & Nationwide & Nationwide & Lombardy & Nationwide & Nationwide & Nationwide \\
\hline Population & $\begin{array}{c}\text { Adults }(\geq 20 \\
\text { years) }\end{array}$ & $\begin{array}{l}\text { No age } \\
\text { restriction }\end{array}$ & $\begin{array}{l}\text { No age } \\
\text { restriction }\end{array}$ & $\geq 18$ years & $\geq 18$ years & $\geq 18$ years \\
\hline $\begin{array}{l}\text { Hospitalized } \\
\text { patients }(\mathrm{N})\end{array}$ & 232,036 & 20,133 & 3,988 & 2,215 & 10,021 & 131,583 \\
\hline $\begin{array}{l}\text { ICU } \\
\text { patients } \\
\text { analyzed } \\
\text { (N) }\end{array}$ & $79,687 / 205,493$ & 3,001 & 3,988 & 2,215 & Not reported & Not reported \\
\hline $\begin{array}{l}\text { Patients } \\
\text { with } \\
\text { invasive } \\
\text { mechanical } \\
\text { ventilation } \\
\text { (N) }\end{array}$ & $\begin{array}{l}45,205 / \\
196,248\end{array}$ & 1,658 & 2,929 & 1,494 & 1,318 & 12,018 \\
\hline $\begin{array}{l}\text { Age } \\
\text { (median, } \\
\text { IQR or } \\
\text { mean, SD) }\end{array}$ & $61(47-73)$ & $72 \cdot 9(58-82)$ & $63(56-69)$ & $60.5(14.5)$ & $72(57-82)$ & Not reported \\
\hline Male (\%) & $56 \%$ & $60 \%$ & $79.9 \%$ & $64.8 \%$ & $51.9 \%$ & Not reported \\
\hline $\begin{array}{l}\text { Patients } \\
\text { that } \\
\text { remained } \\
\text { hospitalized } \\
\text { at the end } \\
\text { of study or } \\
\text { incomplete } \\
\text { data }(\mathrm{N}, \%)\end{array}$ & $22,252(8 \cdot 8 \%)$ & $6,769(34 \%)$ & $501(12 \cdot 6 \%)$ & $137(6 \cdot 2 \%)$ & $4 \cdot 8 \%$ & Not reported \\
\hline $\begin{array}{l}\text { In-hospital } \\
\text { mortality of } \\
\text { patients } \\
\text { with a } \\
\text { hospital } \\
\text { discharge } \\
\text { (\%) }\end{array}$ & $38 \%$ & $\begin{array}{c}5165 / 13,364 \\
(39 \%)\end{array}$ & Not reported & $35 \cdot 4 \%$ & $22 \%$ & Not reported \\
\hline $\begin{array}{l}\text { In-hospital } \\
\text { mortality of } \\
\text { ICU } \\
\text { patients } \\
\text { with } \\
\text { discharge } \\
\text { disposition } \\
\text { at study end } \\
\text { (\%) }\end{array}$ & $59 \%$ & $54 \%$ & $48 \cdot 3 \%$ & $39.5 \%$ & Not reported & Not reported \\
\hline $\begin{array}{l}\text { Mortality in } \\
\text { patients } \\
\text { with } \\
\text { invasive } \\
\text { mechanical } \\
\text { ventilation } \\
\text { (\%) }\end{array}$ & $\begin{array}{c}80 \cdot 0 \% \\
(36,046 / 45,205)\end{array}$ & $69 \%$ & $51.7 \%$ & $\begin{array}{l}\text { Not } \\
\text { reported }\end{array}$ & $52 \cdot 8 \%$ & $73.7 \%$ \\
\hline
\end{tabular}


12.4

Appendix A5

\subsection{1}

\section{Appendix A5.1 - Data sources and study population}

Data from hospital admissions were obtained from the Influenza Epidemiological Surveillance Information System, SIVEP-Gripe (Sistema de Informação de Vigilância Epidemiológica da Gripe), a nationwide surveillance database used to monitor severe acute respiratory infections in Brazil. A detailed description of data definition was provided previously (RANZANI et al., 2021)

Briefly, we included patients with COVID-19 diagnosed by RT-qPCR or other criteria, aged over 20 years, an admitted to the hospital between February 16, 2020 to May 24, 2021.

In our analysis, we compared first, and second waves defined by the lowest value per week of hospitalized cases in Brazil (Epidemiological week 43). ${ }^{2}$ Within the second wave, we compared the periods before and after the dominance (when genomic data point towards $50 \%$ of the sample carrying the mutation, epidemiological week 53).

In Appendix A4.2, we also considered data of prevalence for the P.1 and B.1.1.7 variants of concern (LATIF et al., 2020).

\subsection{2}

\section{Appendix A5.2 - Average daily prevalence of Variants of Concern in Brazil}

Shaded area: Noisy data due to small samples reported, according to https://outbreak.info

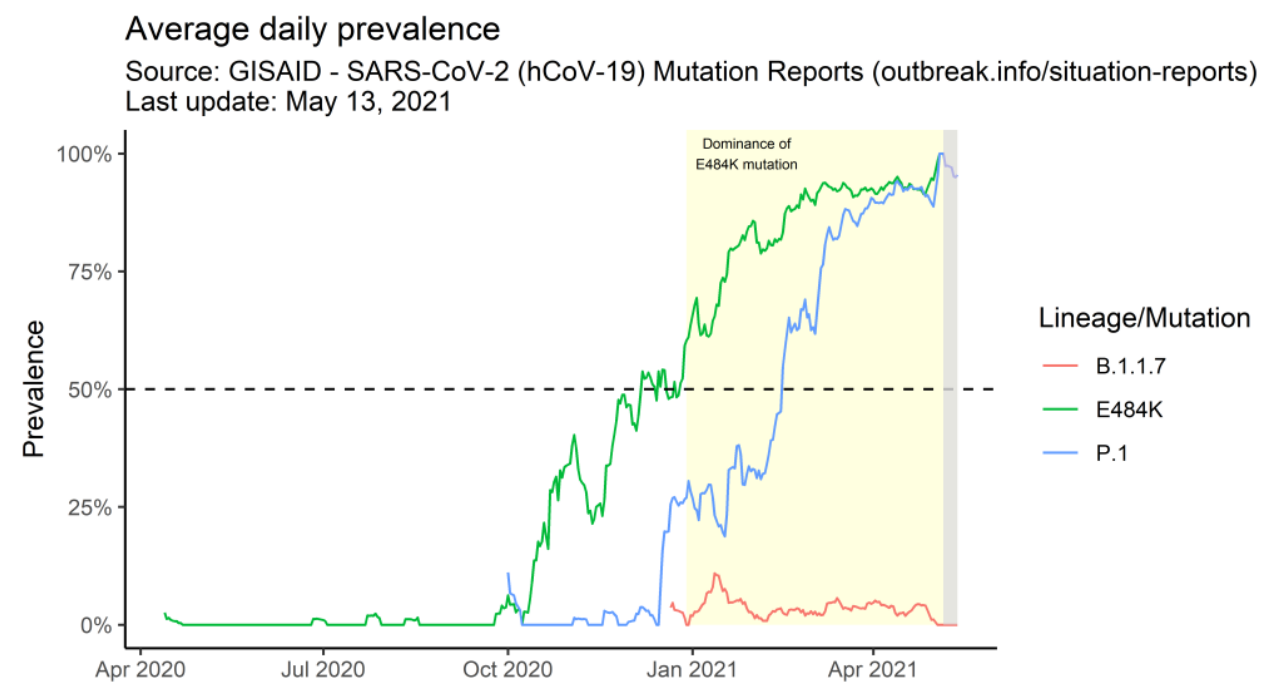


12.4.3

Appendix A5.3 - Comparison of hospital admissions and in-hospital
mortality between first and second COVID-19 waves in Brazil ( $n=$
$1,187,840)$

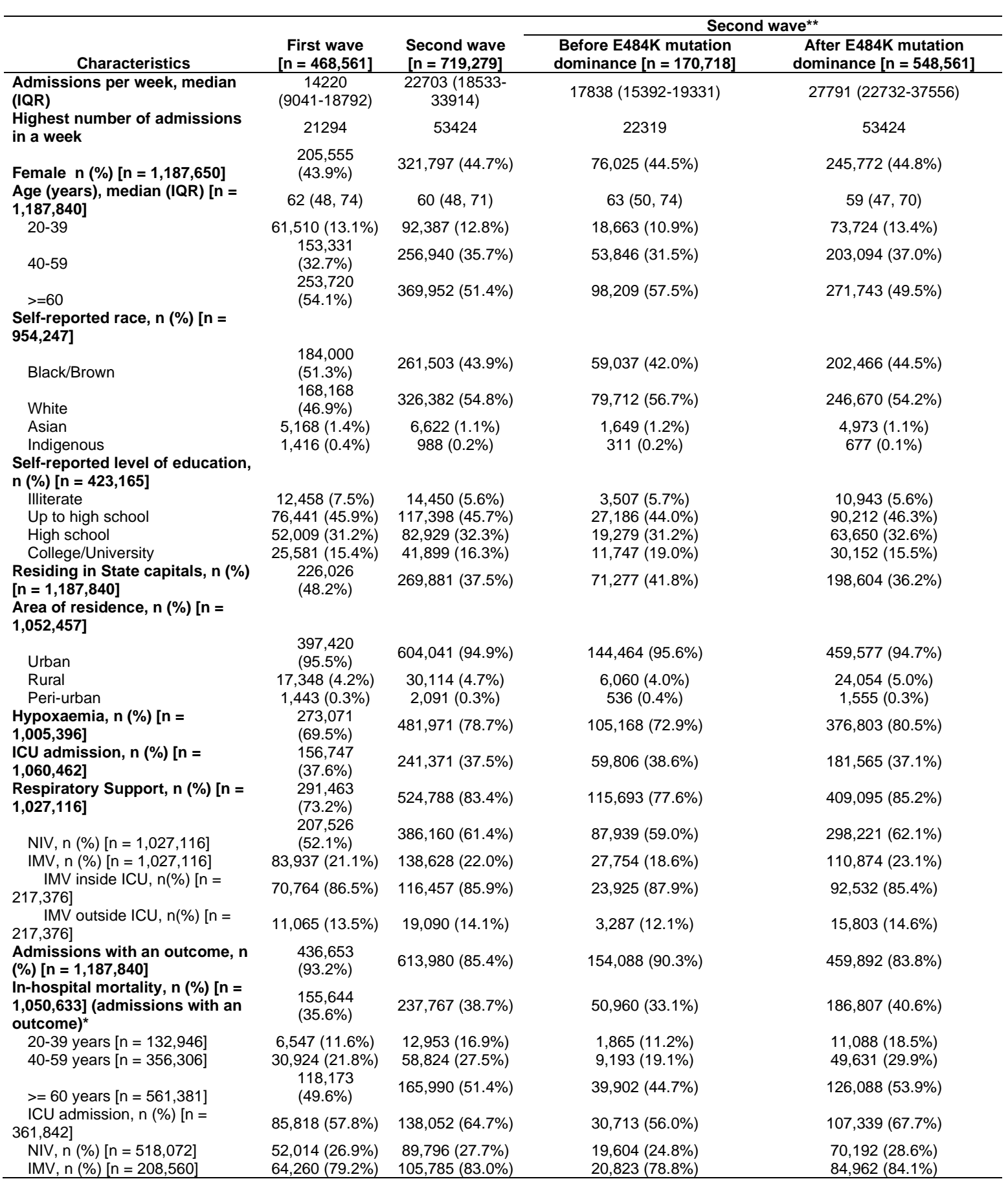

ICU - intensive care unit; NIV - Non-invasive ventilation; IMV - Invasive Mechanical Ventilation

*All in-hospital mortality estimates were calculated using only admissions with an outcome.

First wave - Epidemiological weeks 8/2020 to 43/2020 (February 16, 2020 to October 24, 2020)

Second wave - Epidemiological weeks $44 / 2020$ to $17 / 2021^{*}$ (October 25, 2020 to May 01, 2021)

**We included data until week 16/2021 (May 01, 2021) to reduce potential effects from the notification delay on estimates.

Before E484K mutation dominance - Epidemiological weeks 44/2020 to 53/2020 (October 25, 2020 to January 02, 2021)

After E484K mutation dominance - Epidemiological weeks 01/2021 to 16/2021 


\subsection{4}

Appendix A5.4 - Comparison of first and second waves of COVID-19 hospital admissions in Brazil.

We compared the caseload of hospital admissions since the beginning of each wave: first wave - week 8, second wave - week 43. Dashed line represents expected delay in notification of hospital admissions to the SIVEP-Gripe database

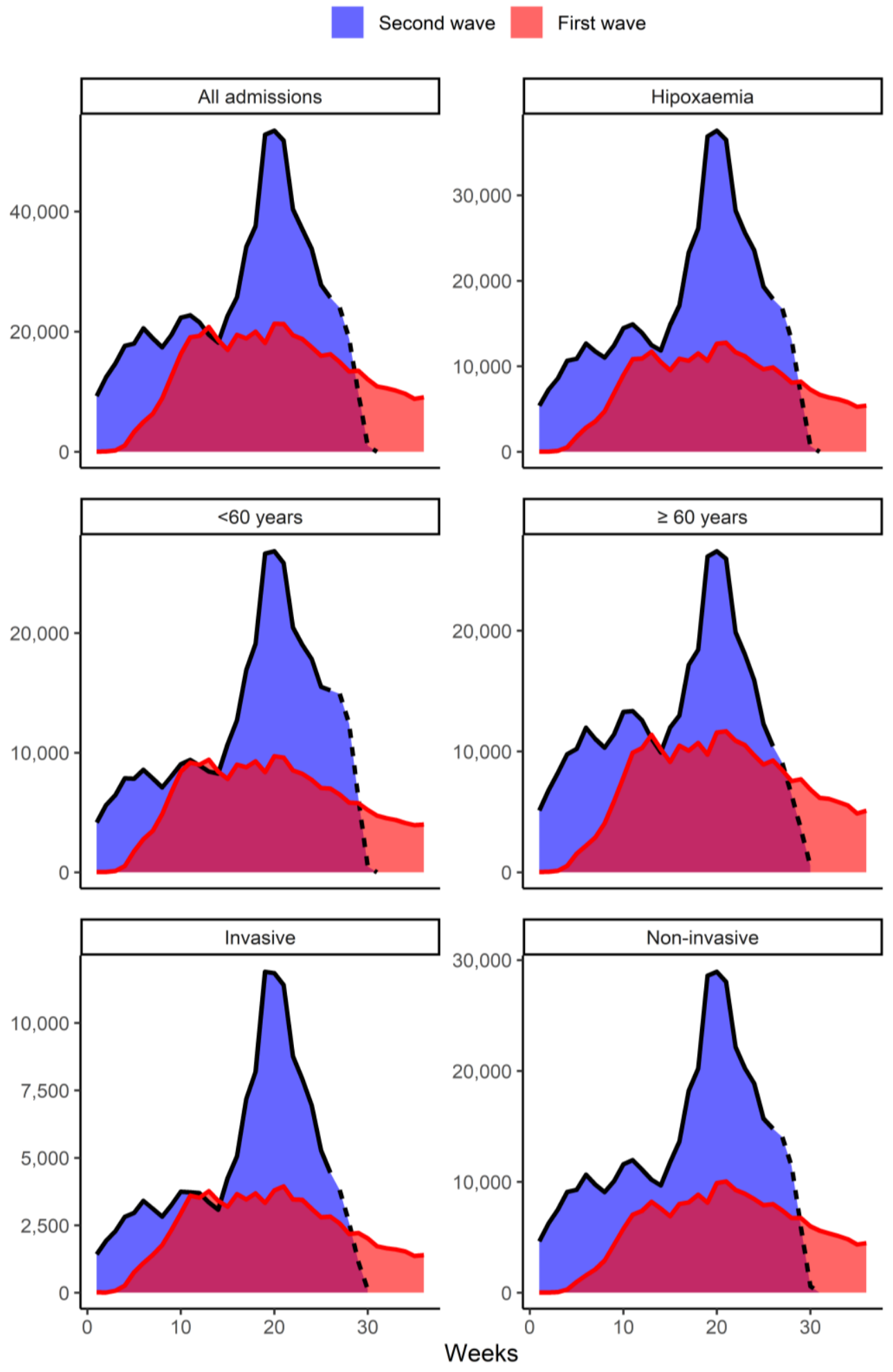




\subsection{5}

\section{Appendix A5.5 - In-hospital mortality stratified by hypoxaemia, age, and respiratory support.}

Data refers to adult COVID-19 hospital admissions with an outcome. The $\mathrm{x}$-axis denotes the epidemiological week when symptom onset occurred for hospital admissions. The grey-shaded area represents a period of uncertainty, particularly for deaths, due to the expected notification delay from the SIVEP-Gripe (Data exported on May 26, 2021). First and second waves are defined by the lowest value per week of hospitalized cases in Brazil (dashed line, epidemiological week 43/2020),3 whereas the yellow-shaded area in the period of the mutation's domination (epidemiological week 53/2020).
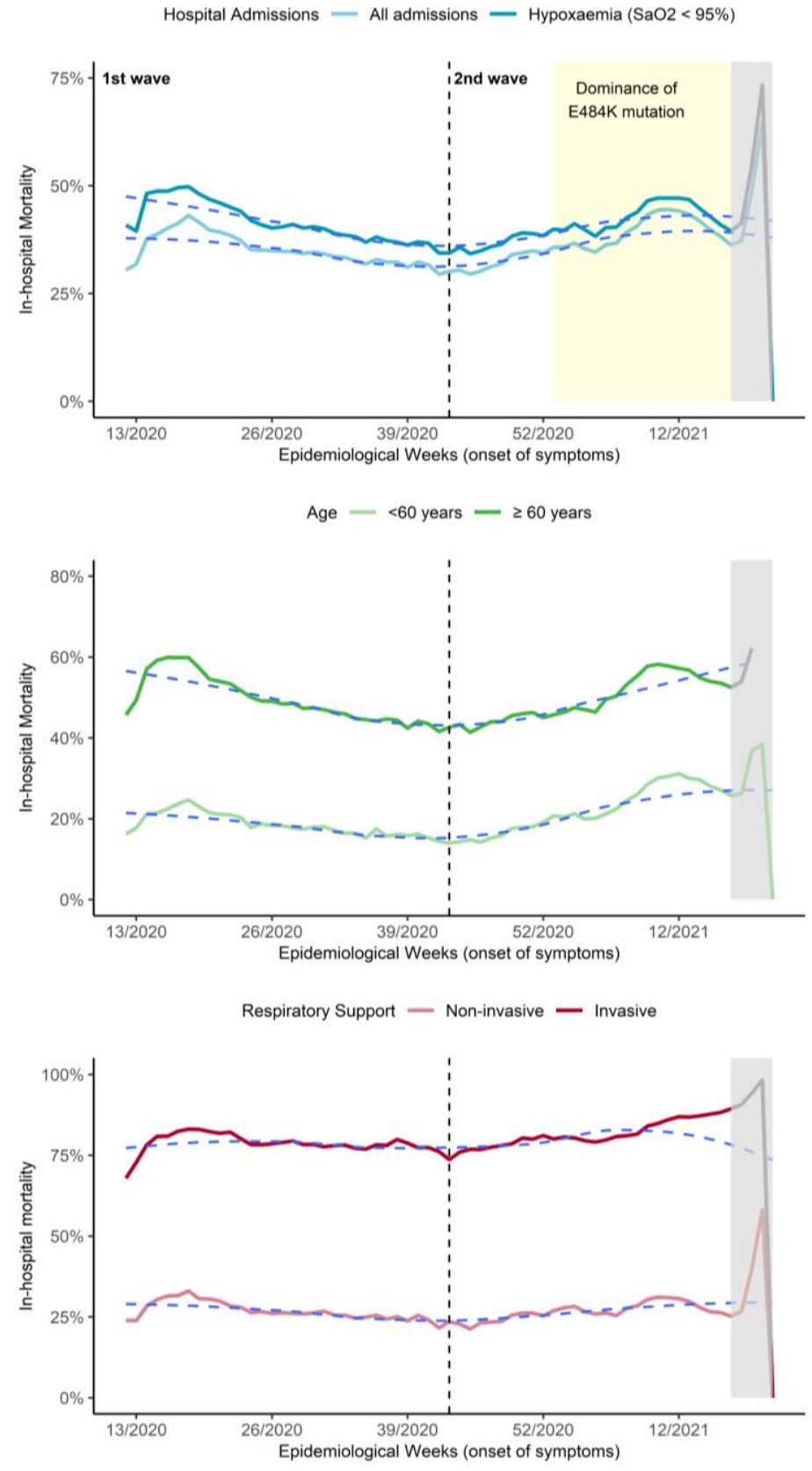


\subsection{6}

\section{Appendix A5.6 - Changes in Mobility in Brazil}

Data extracted from the Google COVID-19 Community Mobility Reports on May 23, 2021. Last update was May 26, 2021.

Mobility is evaluated as the percent change from baseline values of length of stay and visits to different places. Baselines are the median values of each day of the week from January 03, 2020 to February 06, 2020 (GOOGLE LLC, 2020).

We calculated the 7-day moving average of change from daily baseline to reduce noisy data.

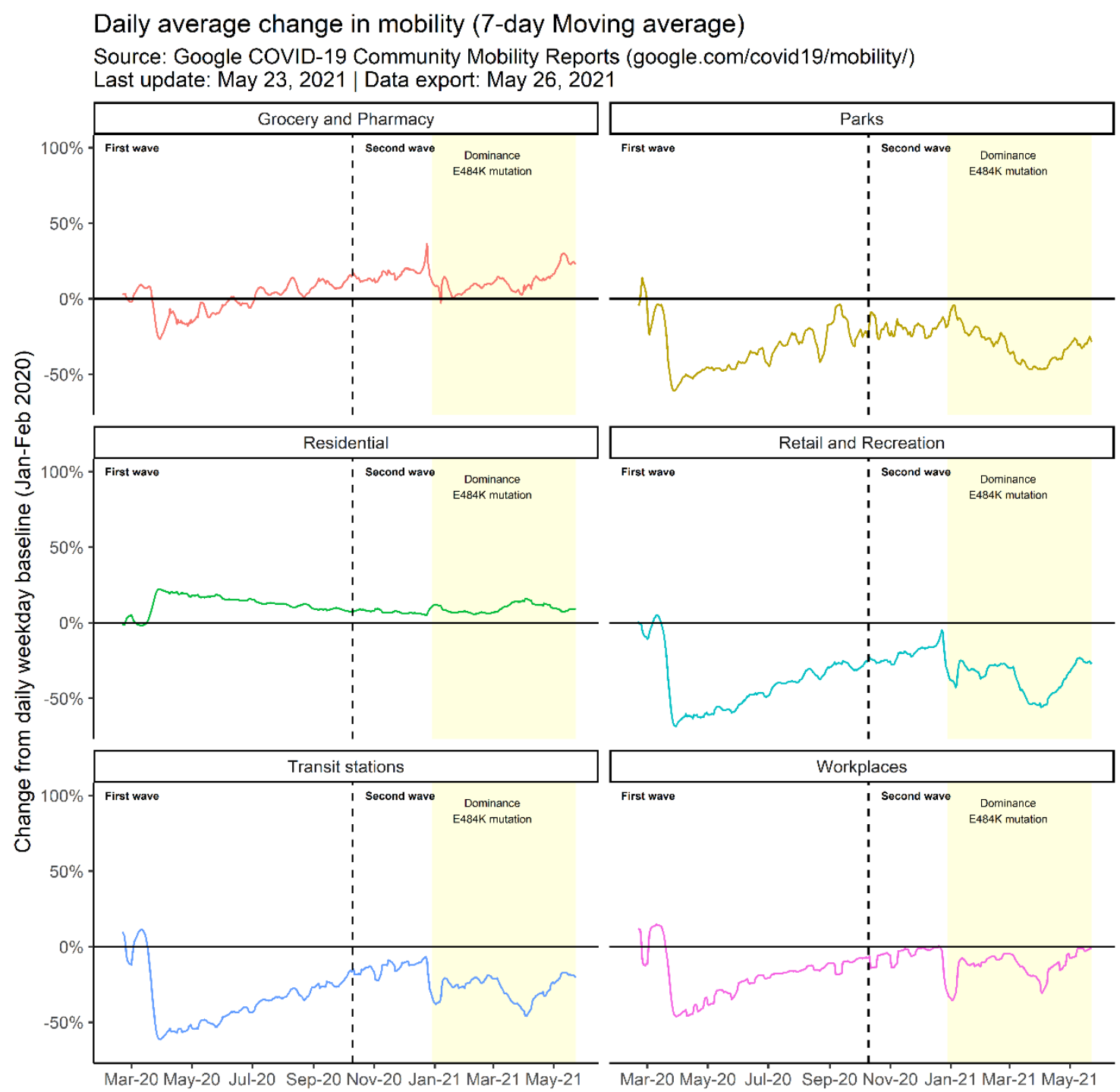




\subsection{1}

\section{Appendix A6.1 - Supplemental Methods}

Missing values: To describe patient's clinical characteristics, outcomes, and organ support, we provided their corresponding number of complete cases for incomplete variables. Those variables were not included in the primary analyses. In sensitivity analysis, we imputed values of $\mathrm{PaFO}_{2} / \mathrm{FiO}_{2}$ ratio using multiple imputation with chained equations.

Structure change identification: To select time periods of different structural behaviors (e.g., trends or inflection points), we used a method for automatic identification of structural changes in time series. This method stratifies the time series and estimates linear models in the segmented data, thus finding the optimal number of breakpoints that minimizes the Bayesian Information Criteria (BIC). The method if available in the $\mathrm{R}$ package strucchange (ZEILEIS et al., 2002).

We evaluated the structural changes in the time series of daily number of ICU deaths. Since there is a delay in the deaths related to the admission, we considered only the time series starting at the occurrence of first death (in this case, March $15^{\text {th }}$ ). The estimated breakpoint dates divided our sample into time periods referred to as "period of admission".

In our study, three breakpoints were estimated (Figure 1 in the manuscript): April $25^{\text {th }}, 2020$; June $06^{\text {th }}, 2020$; and August $10^{\text {th }}$. Thus, our sample was stratified into four distinct time periods, separated by those breakpoints: Period $1-$ February $27^{\text {th }}$ to April $25^{\text {th }}$; Period $2-$ April $26^{\text {th }}$ to June $6^{\text {th }}$; Period $3-$ June $7^{\text {th }}$ to August $10^{\text {th }} ;$ Period 4 - August $11^{\text {th }}$ to October $28^{\text {th }}$.

Random-effects Cox regression model: We fitted a random-effects cox proportional hazards (frailty) model to evaluate the association between clinically relevant variables and 60-day in-hospital death.

The selected variables were: Age [<40, 40-49, 50-59, 60-69, 70-79, 80+ years]; Sex [Female, Male]; Frailty [Modified frailty Index $(\mathrm{MFI})=0, \mathrm{MFI}=1$ or 2, MFI > 3]; Simplified Acute Physiology Score (SAPS-3) quartile at admission [categorical]; Sequential Organ Failure Assessment (SOFA) at admission [continuous]; Admission from an emergency department [indicator]; presence of comorbidities [indicator]: 
Hypertension, Diabetes, Obesity, COPD or Asthma, and Cardiovascular disease; Use of Vasopressor [indicator] and renal replacement therapy [indicator] during the ICU stay; the first respiratory support used [noninvasive respiratory support - NIRS or invasive mechanical ventilation - IMV], and the time period of admission defined by the estimated breakpoints.

Outcome: In-hospital mortality in 60 days after ICU admission (60-day in-hospital mortality)

Multivariable cox final model: Death at day $60 \sim$ Age + Sex + MFI + SAPS-3 + SOFA + Admission from an emergency department + Hypertension + Diabetes + Obesity + COPD or Asthma + Cardiovascular disease + Vasopressor during ICU stay + Renal Replacement Therapy during ICU stay + First respiratory support (NIRS or IMV) + Period of Admission + (1| Hospital $)$

This model syntax means that death in 60 days was to be predicted for several fixed effects (outside parenthesis) and one random effect, which was a random intercept for the Hospital.

Propensity score model: We used propensity score inverse probability treatment weighting (IPTW) to account for the nonrandomization of data in the mortality model.

Propensity score were obtained using a multivariable logistic regression to estimate the probability for a patient to use NIRS as the first respiratory support strategy as opposed to IMV (binary exposure variable: NIRS first $=1$, IMV first $=0$ ).

As predictors, we considered variables that were clinically relevant to the decision of NIRS: Age [<40, 40-49, 50-59, 60-69, 70-79, 80+ years]; Sex [Female, Male]; Frailty [Modified frailty Index $(\mathrm{MFI})=0, \mathrm{MFI}=1$ or 2, MFI > 3]; Sequential Organ Failure Assessment (SOFA) at admission [continuous]; Admission from an emergency department [indicator]; presence of comorbidities [indicator]: Hypertension, Diabetes, Obesity, COPD or Asthma, and Cardiovascular disease; Use of Vasopressor [indicator] and renal replacement therapy [indicator] in the first 24 hours after ICU admission; and the time period of admission defined by the estimated breakpoints. We evaluated the overlapping assumption of propensity scores using density and histogram plots.

Propensity scores final model: First respiratory support (NIRS or IMV) Age + Sex + MFI + SOFA + Admission from an emergency department + Cardiovascular disease + Vasopressor in the first $24 \mathrm{~h}$ after admissions

We performed all analysis in R 4.0.2, using packages: dplyr (WICKHAM et al., [s.d.]) and ggplot2 (HADLEY WICKHAM, [s.d.]) from the tidyverse (WICKHAM et al., 
2019), survival (THERNEAU et al., [s.d.]), WeightIt (GREIFER, 2020), and coxme (THERNEAU, TERRY M., [s.d.]).

12.5.2

Appendix A6.2 - Summary of hospitals and ICUs

Table A12.36 -List of Hospitals

\begin{tabular}{|c|c|c|}
\hline Hospital Name & State & City \\
\hline Instituto Cardio Pulmonar & Bahia & Salvador \\
\hline Hospital Santa Helena & Distrito Federal & Brasília \\
\hline São Luiz Hospital DF Star & Distrito Federal & Brasília \\
\hline Hospital do Coração do Brasil & Distrito Federal & Brasília \\
\hline Hospital Santa Luzia & Distrito Federal & Brasília \\
\hline UDI Hospital & Maranhão & São Luís \\
\hline Hospital Esperança Olinda & Pernambuco & Olinda \\
\hline Hospital Memorial Sao Jose & Pernambuco & Recife \\
\hline Hospital Esperança & Pernambuco & Recife \\
\hline Hospital Sao Marcos & Pernambuco & Recife \\
\hline Caxias D'Or & Rio de Janeiro & Duque de Caxias \\
\hline Niteroi D’Or & Rio de Janeiro & Niteroi \\
\hline Samer Hospital - Serviço de Assistência Médica de Resende & Rio de Janeiro & Resende \\
\hline Hospital Badim & Rio de Janeiro & Rio de Janeiro \\
\hline Hospital Israelita Albert Sabin & Rio de Janeiro & Rio de Janeiro \\
\hline Norte D'Or & Rio de Janeiro & Rio de Janeiro \\
\hline Hospital Oeste D'Or & Rio de Janeiro & Rio de Janeiro \\
\hline Hospital Bangu & Rio de Janeiro & Rio de Janeiro \\
\hline Copa Star & Rio de Janeiro & Rio de Janeiro \\
\hline Hospital Rio Mar & Rio de Janeiro & Rio de Janeiro \\
\hline Hospital Gloria D’Or & Rio de Janeiro & Rio de Janeiro \\
\hline Copa D'Or & Rio de Janeiro & Rio de Janeiro \\
\hline Clínica Sao Vicente & Rio de Janeiro & Rio de Janeiro \\
\hline Hospital Quinta D'Or & Rio de Janeiro & Rio de Janeiro \\
\hline Barra D'Or & Rio de Janeiro & Rio de Janeiro \\
\hline Rios D'Or & Rio de Janeiro & Rio de Janeiro \\
\hline Hospital Alphamed & São Paulo & Carapicuíba \\
\hline Hospital Sino Brasileiro & São Paulo & Osasco \\
\hline Hospital Ribeirao Pires & São Paulo & Ribeirão Pires \\
\hline Hospital Bartira & São Paulo & Santo André \\
\hline Hospital e Maternidade Brasil & São Paulo & Santo André \\
\hline Hospital Assunção & São Paulo & São Bernardo do Campo \\
\hline Hospital e Maternidade São Luiz - São Caetano & São Paulo & São Caetano do Sul \\
\hline Hospital Vivalle & São Paulo & São José dos Campos \\
\hline Hospital Aviccena & São Paulo & São Paulo \\
\hline Hospital São Luiz - Unidade Jabaquara & São Paulo & São Paulo \\
\hline Hospital Villa-Lobos & São Paulo & São Paulo \\
\hline Hospital Vila Nova Star & São Paulo & São Paulo \\
\hline Hospital São Luiz Analia Franco & São Paulo & São Paulo \\
\hline Hospital e Maternidade São Luiz - Unidade Itaim & São Paulo & São Paulo \\
\hline Hospital São Luiz - Unidade Morumbi & São Paulo & São Paulo \\
\hline Hospital Sao Lucas - Aracaju & Sergipe & Aracaju \\
\hline
\end{tabular}


Figure A12.10 - Map of hospital locations

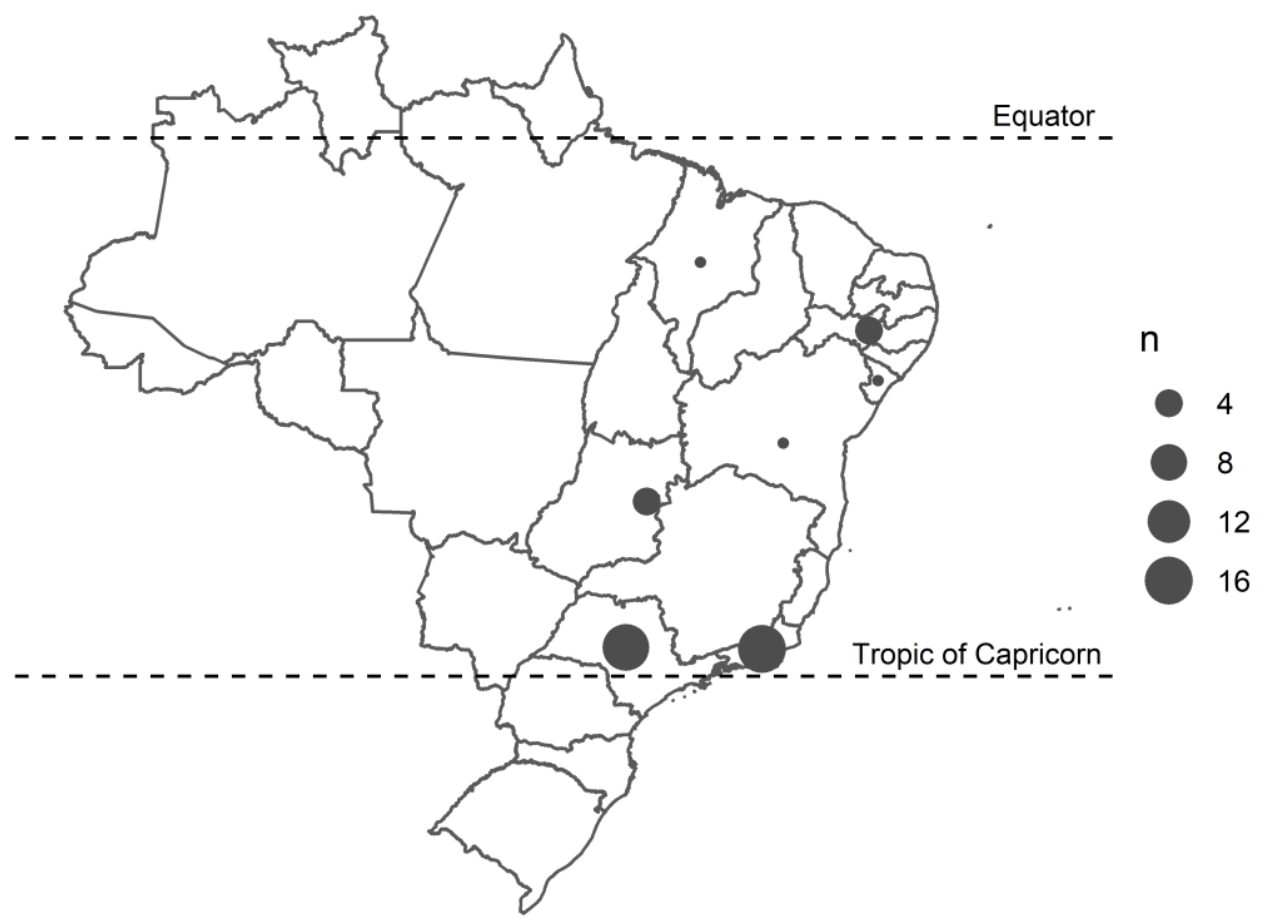

\subsection{3}

Appendix A6.3 - Study Flowchart and ICU occupancy rates

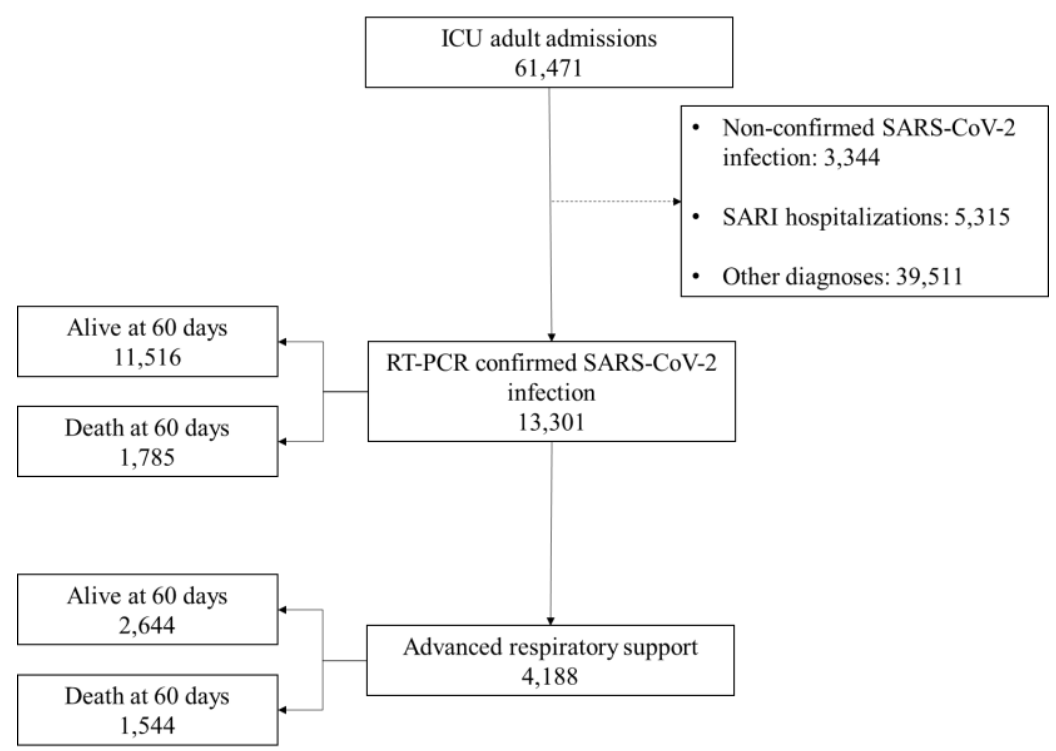

Figure A12.11- Flowchart of the study population.

From February $27^{\text {th }}, 2020$, to July $28^{\text {th }}, 2020,61,471$ patients were admitted to the ICU, from which 13,301 (22\%) were patients with COVID-19, and 4,188 (31\%) underwent advanced respiratory support (Initial advanced respiratory support: noninvasive respiratory support first [NIRS first]: 2,423; invasive mechanical ventilation first [IMV first]: 1,765). 
Table A12.37 - Number of beds; ICU occupancy rates and proportion of patients in invasive mechanical ventilation before and after the pandemic

\begin{tabular}{|c|c|c|c|}
\hline Period & ICU Beds & Occupancy Rate & \\
\hline \multicolumn{4}{|l|}{ October/2019 } \\
\hline Start $\left(1^{\text {st }}\right)$ & 1,692 & $87.2 \%$ & \\
\hline End $\left(31^{\mathrm{st}}\right)$ & 1,730 & $86.4 \%$ & \\
\hline \multicolumn{4}{|l|}{ November/2019 } \\
\hline Start $\left(1^{\text {st }}\right)$ & 1,740 & $83.9 \%$ & \\
\hline End $\left(30^{\text {th }}\right)$ & 1,754 & $75.9 \%$ & \\
\hline \multicolumn{4}{|l|}{ December/2019 } \\
\hline Start $\left(1^{\text {st }}\right)$ & 1,754 & $76.5 \%$ & \\
\hline End $\left(31^{\mathrm{st}}\right)$ & 1,694 & $64.5 \%$ & \\
\hline \multicolumn{4}{|l|}{ January/2019 } \\
\hline Start $\left(1^{\mathrm{st}}\right)$ & 1,694 & $65.2 \%$ & \\
\hline End $\left(31^{\text {st }}\right)$ & 1,803 & $83.5 \%$ & \\
\hline Average & 1,733 & $77.9 \%$ & \\
\hline Peak (May 13 ${ }^{\text {th, }}$ 2020) & Beds/Patients & & \% Patients in IMV \\
\hline Overall available beds & 2,264 & $85.6 \%$ & $495(25.5 \%)$ \\
\hline Non-COVID-19 (bed occupied) & 872 & $38.5 \%$ & $119(13.6 \%)$ \\
\hline COVID-19 (beds occupied) & 1066 & $47.1 \%$ & $376(35.3 \%)$ \\
\hline
\end{tabular}

IMV - Invasive Mechanical Ventilation

Proportion of admissions per diagnostics stratified by the time periods estimated using breakpoints

\begin{tabular}{cccccc}
\hline $\begin{array}{c}\text { Diagnostic } \\
\text { (All Admissions) }\end{array}$ & Period 1 & Period 2 & Period 3 & Period 4 & Total \\
\hline COVID-19 & $2,184(17 \%)$ & $3,536(37 \%)$ & $3,938(23 \%)$ & $3,643(16 \%)$ & $13,301(22 \%)$ \\
Non-COVID-19 & $10,620(83 \%)$ & $5,921(63 \%)$ & $13,214(77 \%)$ & $18,415(84 \%)$ & $48,170(78 \%)$ \\
Total & 12,804 & 9,457 & 17,152 & 22,058 & 61,471 \\
\hline
\end{tabular}

Proportion of admissions per diagnostics stratified by the time periods estimated using breakpoints and that underwent advanced respiratory support (noninvasive or invasive)

\begin{tabular}{cccccc}
\hline $\begin{array}{c}\text { Diagnostic } \\
\text { (Advanced } \\
\text { Resp. Support) }\end{array}$ & Period 1 & Period 2 & Period 3 & Period 4 & Total \\
\hline COVID-19 & $727(36 \%)$ & $1,264(60 \%)$ & $1,058(42 \%)$ & $1,139(35 \%)$ & $4,188(58 \%)$ \\
Non-COVID-19 & $1,310(64 \%)$ & $854(40 \%)$ & $1,462(58 \%)$ & $2,097(65 \%)$ & $5,723(42 \%)$ \\
Total & 2,037 & 2,118 & 2,520 & 3,236 & 9,911 \\
\hline
\end{tabular}


12.5.4

Appendix A6.4 - Additional descriptive analyses

Table A12.38 - Clinical characteristics and outcomes of 4,188 critically ill COVID-19 patients that required advanced respiratory support (noninvasive or invasive). Period $1-$ February $27^{\text {th }}$ to April $25^{\text {th }}$; Period $2-$ April $26^{\text {th }}$ to June $6^{\text {th }}$; Period 3 - June $7^{\text {th }}$ to August $10^{\text {th }}$; Period $4-$ August $11^{\text {th }}$ to October $28^{\text {th }}$

\begin{tabular}{|c|c|c|c|c|c|}
\hline Characteristics & $\begin{array}{c}\text { Total } \\
{[n=4,188]}\end{array}$ & $\begin{array}{l}\text { Period 1 } \\
{[n=727]}\end{array}$ & $\begin{array}{c}\text { Period 2 } \\
{[n=1,264]}\end{array}$ & $\begin{array}{c}\text { Period } 3 \\
{[n=1,058]}\end{array}$ & $\begin{array}{c}\text { Period } 4 \\
{[n=1,139]}\end{array}$ \\
\hline Age, median (IQR) & $63(49,76)$ & $64(52,76)$ & $65(51,79)$ & $61(48,75)$ & $61(47,73)$ \\
\hline$<40$ & $482(12 \%)$ & $70(9.6 \%)$ & $116(9.2 \%)$ & $132(12 \%)$ & $164(14 \%)$ \\
\hline $40-49$ & $601(14 \%)$ & $89(12 \%)$ & $177(14 \%)$ & $168(16 \%)$ & $167(15 \%)$ \\
\hline $50-59$ & $779(19 \%)$ & $144(20 \%)$ & $223(18 \%)$ & $207(20 \%)$ & $205(18 \%)$ \\
\hline $60-69$ & $840(20 \%)$ & $156(21 \%)$ & $223(18 \%)$ & $207(20 \%)$ & $254(22 \%)$ \\
\hline 70-79 & $696(17 \%)$ & $132(18 \%)$ & $215(17 \%)$ & $170(16 \%)$ & $179(16 \%)$ \\
\hline$\geq 80$ & $790(19 \%)$ & $136(19 \%)$ & $310(25 \%)$ & $174(16 \%)$ & $170(15 \%)$ \\
\hline \multicolumn{6}{|l|}{ Sex, No. (\%) } \\
\hline Female & $1516(36 \%)$ & $243(33 \%)$ & $482(38 \%)$ & $380(36 \%)$ & $411(36 \%)$ \\
\hline Male & $2672(64 \%)$ & $484(67 \%)$ & $782(62 \%)$ & $678(64 \%)$ & $728(64 \%)$ \\
\hline Admissions from emergency department, №. (\%) & $2848(68 \%)$ & $469(65 \%)$ & $810(64 \%)$ & $738(70 \%)$ & $831(73 \%)$ \\
\hline \multicolumn{6}{|l|}{ Modified Frailty Index (MFI) } \\
\hline Mean (SD) & $1.52(1.37)$ & $1.61(1.33)$ & $1.66(1.40)$ & $1.43(1.35)$ & $1.40(1.36)$ \\
\hline Median (IQR) & $1(0,2)$ & $1(1,2)$ & $2(1,2)$ & $1(0,2)$ & $1(0,2)$ \\
\hline Non-frail $(M F I=0)$, No. (\%) & $1164(28 \%)$ & $181(25 \%)$ & $308(24 \%)$ & $313(30 \%)$ & $362(32 \%)$ \\
\hline Pre-frail (MFI = 1-2) & $2128(51 \%)$ & $367(50 \%)$ & $642(51 \%)$ & $552(52 \%)$ & $567(50 \%)$ \\
\hline Frail $(\mathrm{MFI}>=3$ ) & $896(21 \%)$ & $179(25 \%)$ & $314(25 \%)$ & $193(18 \%)$ & $210(18 \%)$ \\
\hline SAPS-3, Median (IQR) & $50(42,61)$ & $52(43,64)$ & $53(44,64)$ & $48(41,58)$ & $47(41,57)$ \\
\hline$\leq 42$ & $1,165(28 \%)$ & $158(22 \%)$ & $282(22 \%)$ & $347(33 \%)$ & $378(33 \%)$ \\
\hline $43-50$ & $982(23 \%)$ & $161(22 \%)$ & $262(21 \%)$ & $257(24 \%)$ & $302(27 \%)$ \\
\hline $51-61$ & $1,034(25 \%)$ & $205(28 \%)$ & $339(27 \%)$ & $244(23 \%)$ & $246(22 \%)$ \\
\hline$>61$ & $1,007(24 \%)$ & $203(28 \%)$ & $381(30 \%)$ & $210(20 \%)$ & $213(19 \%)$ \\
\hline SOFA, Median (IQR) & $2(0,5)$ & $3(1,7)$ & $3(1,6)$ & $1(0,4)$ & $1(0,4)$ \\
\hline PaO2/FiO2, No. (\%) $[n=1,963]$ & $170(94,279)$ & $170(89,281)$ & $175(107,276)$ & $168(89,285)$ & $169(88,275)$ \\
\hline Normal $(>300)$ & $431(22 \%)$ & $86(21 \%)$ & $149(22 \%)$ & $101(24 \%)$ & $95(21 \%)$ \\
\hline Mild (201-300) & $385(20 \%)$ & $75(19 \%)$ & $139(21 \%)$ & $77(18 \%)$ & $94(20 \%)$ \\
\hline Moderate (101-200) & $621(32 \%)$ & $128(32 \%)$ & $229(34 \%)$ & $125(29 \%)$ & $139(30 \%)$ \\
\hline Severe $(\leq 100)$ & $526(27 \%)$ & $116(29 \%)$ & $151(23 \%)$ & $126(29 \%)$ & $133(29 \%)$ \\
\hline \multicolumn{6}{|l|}{ Advanced respiratory support, №. (\%) } \\
\hline Noninvasive respiratory support (NIRS) & $2423(58 \%)$ & $182(25 \%)$ & $567(45 \%)$ & $772(73 \%)$ & $902(79 \%)$ \\
\hline Only NPPV & $2061(85 \%)$ & $168(92 \%)$ & $519(92 \%)$ & $659(85 \%)$ & $715(79 \%)$ \\
\hline Only HFNC & $136(5.6 \%)$ & $8(4.4 \%)$ & $26(4.6 \%)$ & $48(6.2 \%)$ & $54(6.0 \%)$ \\
\hline Both & $226(9.3 \%)$ & $6(3.3 \%)$ & $22(3.9 \%)$ & $65(8.4 \%)$ & $133(15 \%)$ \\
\hline Only NIRS & $1558(37 \%)$ & $84(12 \%)$ & $308(24 \%)$ & $513(48 \%)$ & $653(57 \%)$ \\
\hline NIRS failure & $865(21 \%)$ & $98(13 \%)$ & $259(20 \%)$ & $259(24 \%)$ & $249(22 \%)$ \\
\hline Only IMV & $1765(42 \%)$ & $545(75 \%)$ & $697(55 \%)$ & $286(27 \%)$ & $237(21 \%)$ \\
\hline Vasopressor, No. (\%) & $1890(45 \%)$ & $461(63 \%)$ & $697(55 \%)$ & $383(36 \%)$ & $349(31 \%)$ \\
\hline Renal Replacement Therapy, №. (\%) & $896(21 \%)$ & $239(33 \%)$ & $333(26 \%)$ & $186(18 \%)$ & $138(12 \%)$ \\
\hline \multicolumn{6}{|l|}{ Length-of-stay (LOS), Median (IQR) } \\
\hline ICU $[n=4,185]$ & $12(7,22)$ & $17(9,30)$ & $14(7,23)$ & $11(6,19)$ & $11(6,18)$ \\
\hline Hospital $[n=4,160]$ & $17(10,30)$ & $21(11,38)$ & $18(10,32)$ & $16(9,27)$ & $14(9,25)$ \\
\hline \multicolumn{6}{|l|}{ Hospitalizations with LOS > 7 days, No. (\%) } \\
\hline $\operatorname{ICU}[\mathrm{n}=4,185]$ & 3011 (72\%) & $583(80 \%)$ & $933(74 \%)$ & $724(68 \%)$ & $771(68 \%)$ \\
\hline Hospital $[n=4,160]$ & $3496(84 \%)$ & $619(85 \%)$ & $1077(86 \%)$ & $876(83 \%)$ & $924(82 \%)$ \\
\hline 60-day in-hospital deaths, №. (\%) & $1544(37 \%)$ & $331(46 \%)$ & $569(45 \%)$ & $338(32 \%)$ & $306(27 \%)$ \\
\hline ICU deaths, No. $(\%)[n=4,185]$ & $1329(32 \%)$ & $294(40 \%)$ & $501(40 \%)$ & $292(28 \%)$ & $242(21 \%)$ \\
\hline In-hospital deaths, No. (\%) $[\mathrm{n}=4,160]$ & $1572(38 \%)$ & $336(46 \%)$ & $582(46 \%)$ & $344(33 \%)$ & $310(28 \%)$ \\
\hline Ongoing patients, No. (\%) & $28(0.7 \%)$ & $3(0.4 \%)$ & $5(0.4 \%)$ & $4(0.4 \%)$ & $16(1.4 \%)$ \\
\hline
\end{tabular}

IQR - Interquartile Range; SAPS - Simplified Acute Physiology Score; SOFA - Sequential Organ Failure Assessment; NIRS - Noninvasive Respiratory Support; NPPV - Noninvasive Positive Pressure Ventilation; HFNC - High-Flow Nasal Cannula; IMV - Invasive Mechanical Ventilation; ICU - Intensive care unit 
Table A12.39 - Distribution of patient's comorbidities in overall and stratified by the time periods defined by the estimated breakpoints (Period $1-$ February $27^{\text {th }}$ to April $25^{\text {th }}$; Period $2-$ April $26^{\text {th }}$ to June $6^{\text {th }}$; Period 3 - June $7^{\text {th }}$ to August $10^{\text {th }}$; Period 4 - August $11^{\text {th }}$ to October $28^{\text {th }}$ )

Comorbidities of all patients with COVID-19 in overall and stratified by the time periods defined by the estimated breakpoints $(n=13,301)$.

\begin{tabular}{|c|c|c|c|c|c|}
\hline Comorbidities & $\begin{array}{c}\text { Total } \\
{[n=13,301]}\end{array}$ & $\begin{array}{c}\text { Period 1 } \\
{[n=2,184]}\end{array}$ & $\begin{array}{c}\text { Period 2 } \\
{[n=3,536]}\end{array}$ & $\begin{array}{c}\text { Period } 3 \\
{[n=3,938]}\end{array}$ & $\begin{array}{c}\text { Period } 4 \\
{[n=3,643]}\end{array}$ \\
\hline Any comorbidities, No. (\%) & $9019(68 \%)$ & $1544(71 \%)$ & $2553(72 \%)$ & 2524 (64\%) & $2398(66 \%)$ \\
\hline Hypertension & $5669(43 \%)$ & $998(46 \%)$ & $1689(48 \%)$ & $1497(38 \%)$ & $1485(41 \%)$ \\
\hline Diabetes & 3207 (24\%) & $546(25 \%)$ & $983(28 \%)$ & $868(22 \%)$ & $810(22 \%)$ \\
\hline Immunosuppression & $1796(14 \%)$ & $304(14 \%)$ & $472(13 \%)$ & $522(13 \%)$ & $498(14 \%)$ \\
\hline Cardiovascular disease & $1654(12 \%)$ & $302(14 \%)$ & $546(15 \%)$ & $418(11 \%)$ & $388(11 \%)$ \\
\hline Obesity & $1129(8.5 \%)$ & $160(7.3 \%)$ & $318(9.0 \%)$ & $321(8.2 \%)$ & $330(9.1 \%)$ \\
\hline COPD or Asthma & $980(7.4 \%)$ & $180(8.2 \%)$ & $278(7.9 \%)$ & $251(6.4 \%)$ & $271(7.4 \%)$ \\
\hline Malignancy & $785(5.9 \%)$ & $155(7.1 \%)$ & $227(6.4 \%)$ & $211(5.4 \%)$ & $192(5.3 \%)$ \\
\hline Cerebrovascular disease & $889(6.7 \%)$ & $172(7.9 \%)$ & $343(9.7 \%)$ & $210(5.3 \%)$ & $164(4.5 \%)$ \\
\hline Chronic kidney disease & $648(4.9 \%)$ & $116(5.3 \%)$ & $237(6.7 \%)$ & $176(4.5 \%)$ & $119(3.3 \%)$ \\
\hline Tobacco & $395(3.0 \%)$ & $71(3.3 \%)$ & $120(3.4 \%)$ & $99(2.5 \%)$ & $105(2.9 \%)$ \\
\hline Liver cirrhosis & $68(0.5 \%)$ & $16(0.7 \%)$ & $20(0.6 \%)$ & $20(0.5 \%)$ & $12(0.3 \%)$ \\
\hline Other comorbidities & $2532(19 \%)$ & $393(18 \%)$ & $611(17 \%)$ & $773(20 \%)$ & $755(21 \%)$ \\
\hline \multicolumn{6}{|l|}{$\begin{array}{l}\text { Charlson Comorbidity Index } \\
{[n=13,128]}\end{array}$} \\
\hline Mean (SD) & $0.82(1.53)$ & $0.90(1.66)$ & $0.96(1.59)$ & $0.73(1.45)$ & $0.71(1.46)$ \\
\hline Median (IQR) & $0(0,1)$ & $0(0,1)$ & $0(0,1)$ & $0(0,1)$ & $0(0,1)$ \\
\hline
\end{tabular}

Comorbidities of all patients with COVID-19 that underwent invasive mechanical ventilation or noninvasive respiratory support - in overall and stratified by the time periods defined by the estimated breakpoints $(n=4,188)$.

\begin{tabular}{lccccc}
\hline \multicolumn{1}{c}{ Comorbidities } & $\begin{array}{c}\text { Total } \\
\text { [n = 4,188] }\end{array}$ & $\begin{array}{c}\text { Period 1 } \\
\text { [n= 727] }\end{array}$ & $\begin{array}{c}\text { Period 2 } \\
{[\mathbf{n = 1 , 2 6 4 ]}}\end{array}$ & $\begin{array}{c}\text { Period 3 } \\
{[\mathbf{n = 1 , 0 5 8 ]}}\end{array}$ & $\begin{array}{c}\text { Period 4 } \\
{[\mathbf{n = 1 , 1 3 9 ]}}\end{array}$ \\
\hline Any comorbidities, No. (\%) & $3393(81 \%)$ & $600(83 \%)$ & $1061(84 \%)$ & $843(80 \%)$ & $889(78 \%)$ \\
$\quad$ Hypertension & $2310(55 \%)$ & $438(60 \%)$ & $744(59 \%)$ & $546(52 \%)$ & $582(51 \%)$ \\
Diabetes & $1421(34 \%)$ & $256(35 \%)$ & $449(36 \%)$ & $348(33 \%)$ & $368(32 \%)$ \\
Immunosuppression & $699(17 \%)$ & $111(15 \%)$ & $205(16 \%)$ & $188(18 \%)$ & $195(17 \%)$ \\
Cardiovascular disease & $766(18 \%)$ & $146(20 \%)$ & $255(20 \%)$ & $185(17 \%)$ & $180(16 \%)$ \\
Obesity & $543(13 \%)$ & $67(9.2 \%)$ & $162(13 \%)$ & $156(15 \%)$ & $158(14 \%)$ \\
COPD or Asthma & $419(10 \%)$ & $73(10 \%)$ & $125(9.9 \%)$ & $105(9.9 \%)$ & $116(10 \%)$ \\
Malignancy & $336(8.0 \%)$ & $70(9.6 \%)$ & $106(8.4 \%)$ & $84(7.9 \%)$ & $76(6.7 \%)$ \\
Cerebrovascular disease & $399(9.5 \%)$ & $82(11 \%)$ & $158(12 \%)$ & $81(7.7 \%)$ & $78(6.8 \%)$ \\
Chronic kidney disease & $360(8.6 \%)$ & $68(9.4 \%)$ & $138(11 \%)$ & $93(8.8 \%)$ & $61(5.4 \%)$ \\
Tobacco & $189(4.5 \%)$ & $31(4.3 \%)$ & $68(5.4 \%)$ & $43(4.1 \%)$ & $47(4.1 \%)$ \\
Liver cirrhosis & $44(1.1 \%)$ & $12(1.7 \%)$ & $16(1.3 \%)$ & $6(0.6 \%)$ & $10(0.9 \%)$ \\
$\quad$ Other comorbidities & $944(23 \%)$ & $149(20 \%)$ & $268(21 \%)$ & $266(25 \%)$ & $261(23 \%)$ \\
Charlson Comorbidity Index & & & & & \\
[n = 4,138] & $1.23(1.84)$ & $1.34(1.93)$ & $1.33(1.82)$ & $1.21(1.84)$ & $1.08(1.79)$ \\
$\quad$ Mean (SD) & $1(0,2)$ & $1(0,2)$ & $1(02)$ & $1(0,2)$ & $0(0,1)$ \\
$\quad$ Median (IQR) &
\end{tabular}

COPD - Chronic Obstructive Pulmonary Disease; SD - Standard deviation; IQR - Interquartile Range 
(A)

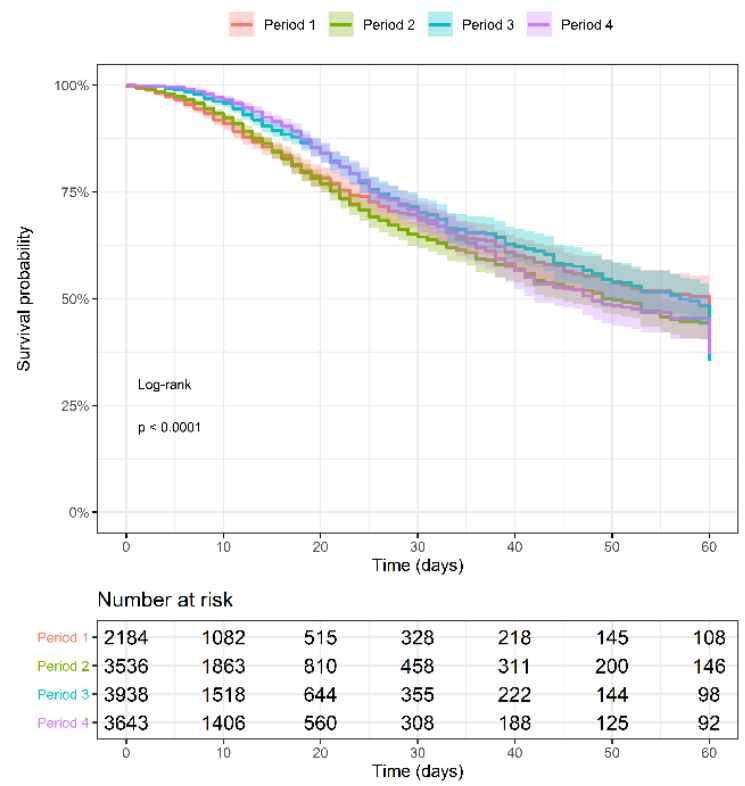

(C)

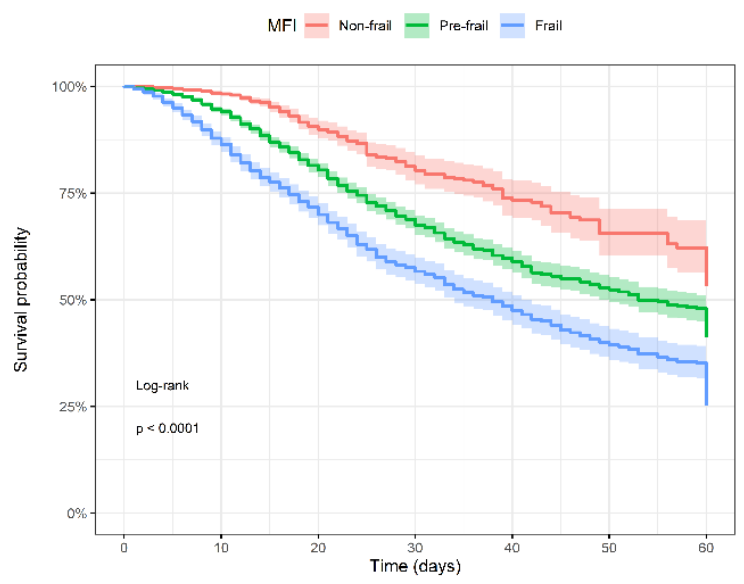

(B)

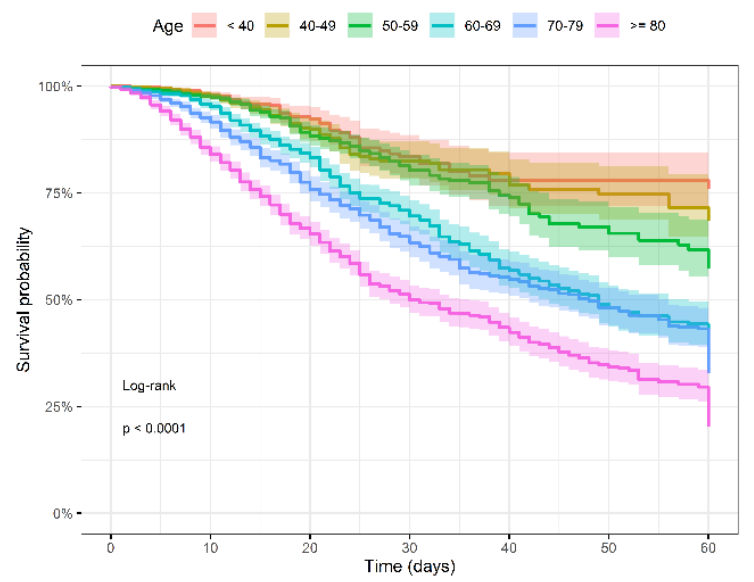

Number at risk

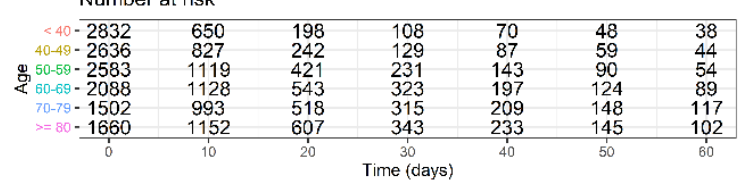

(D)

First Respiratory Support - oxygen - NIRS first - IMV first

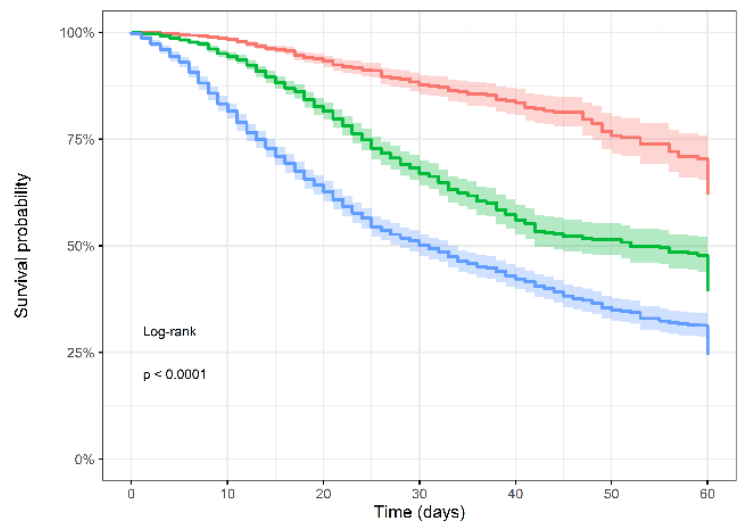

Figure A12.12 - Univariable survival curves (Kaplan-Meier) of factors related to the 60-day outcome in critically ill patients that underwent invasive mechanical ventilation or noninvasive respiratory support $(n=13,301)$.

A - Time periods estimated with the breakpoints of structure change in daily ICU deaths time series (Period 1: February 27 th to April $25^{\text {th }}$; Period: April $26^{\text {th }}$ to June $6^{\text {th }}$; Period 3: June $7^{\text {th }}$ to August $10^{\text {th }}$; Period 4: August $11^{\text {th }}$ to October $\left.28^{\text {th }}\right) ; B$ - Age $(<40,40-49,50-59,60-69,70-79$, $\geq 80$; C - Modified Frailty Index (MFI) at the admission, with groups Non-frail (MFI =0), Pre-frail $(\mathrm{MFI}=1-2)$ and Frail (MFI $\geq 3$ ); $\mathrm{D}$ - First respiratory support considering patients that underwent only oxygen, firstly respiratory support (NIRS first) or invasive ventilation (IMV first). Differences among curves were assessed using the log-rank test with a confidence level of 0.05 . 

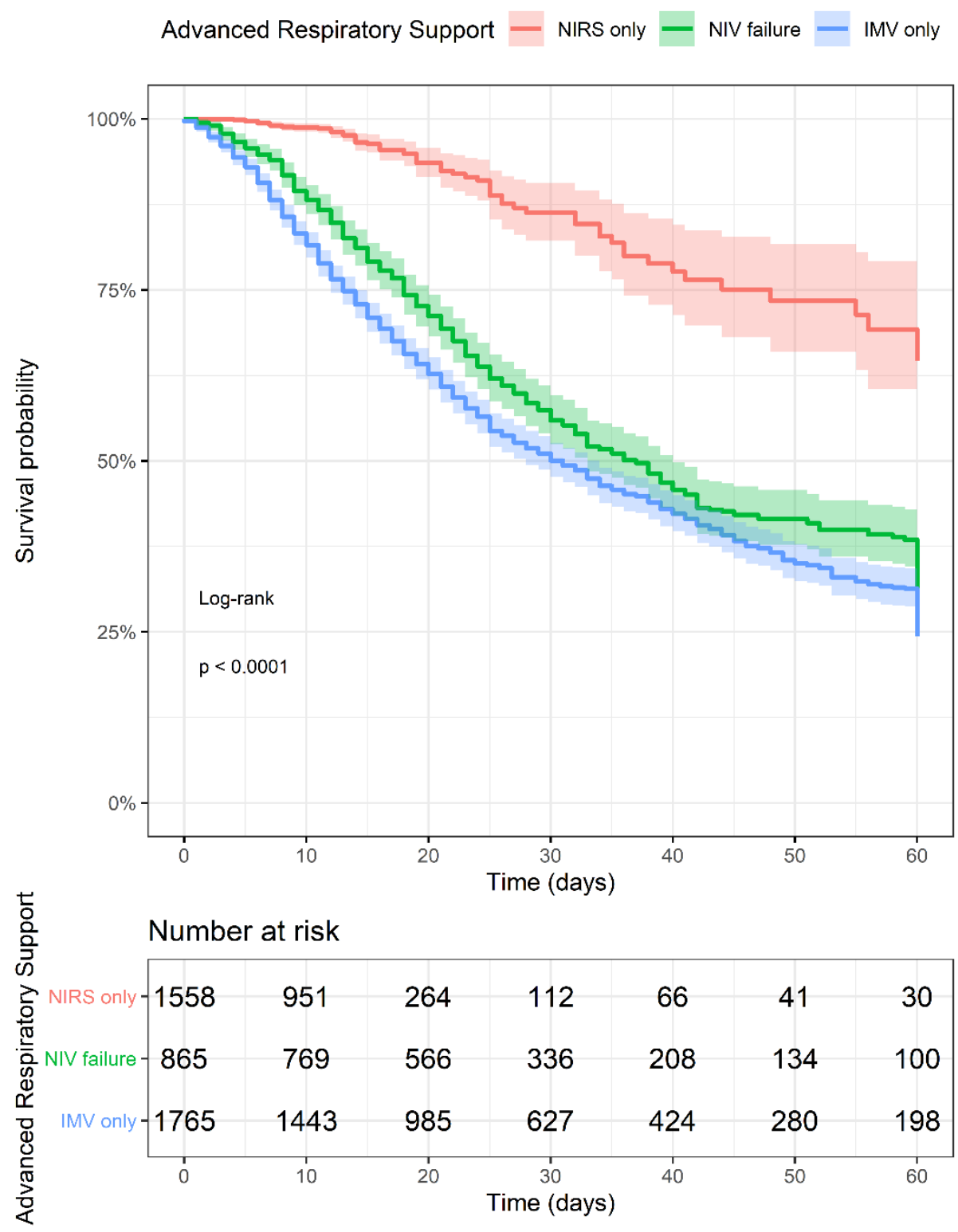

Figure A12.13 - Univariable survival curves (Kaplan-Meier) 60-day outcome in critically ill patients that underwent advanced respiratory support $(n=4,188)$ stratified by Respiratory support considering patients that underwent oxygen support, noninvasive respiratory support (NIRS only), NIRS with subsequent intubation (NIRS failure) and invasive ventilation (IMV only). Differences among curves were assessed using the log-rank test with a confidence level of 0.05 . 


\section{5 .5}

Appendix A6.5 - Modelling preparations, propensity scores and results
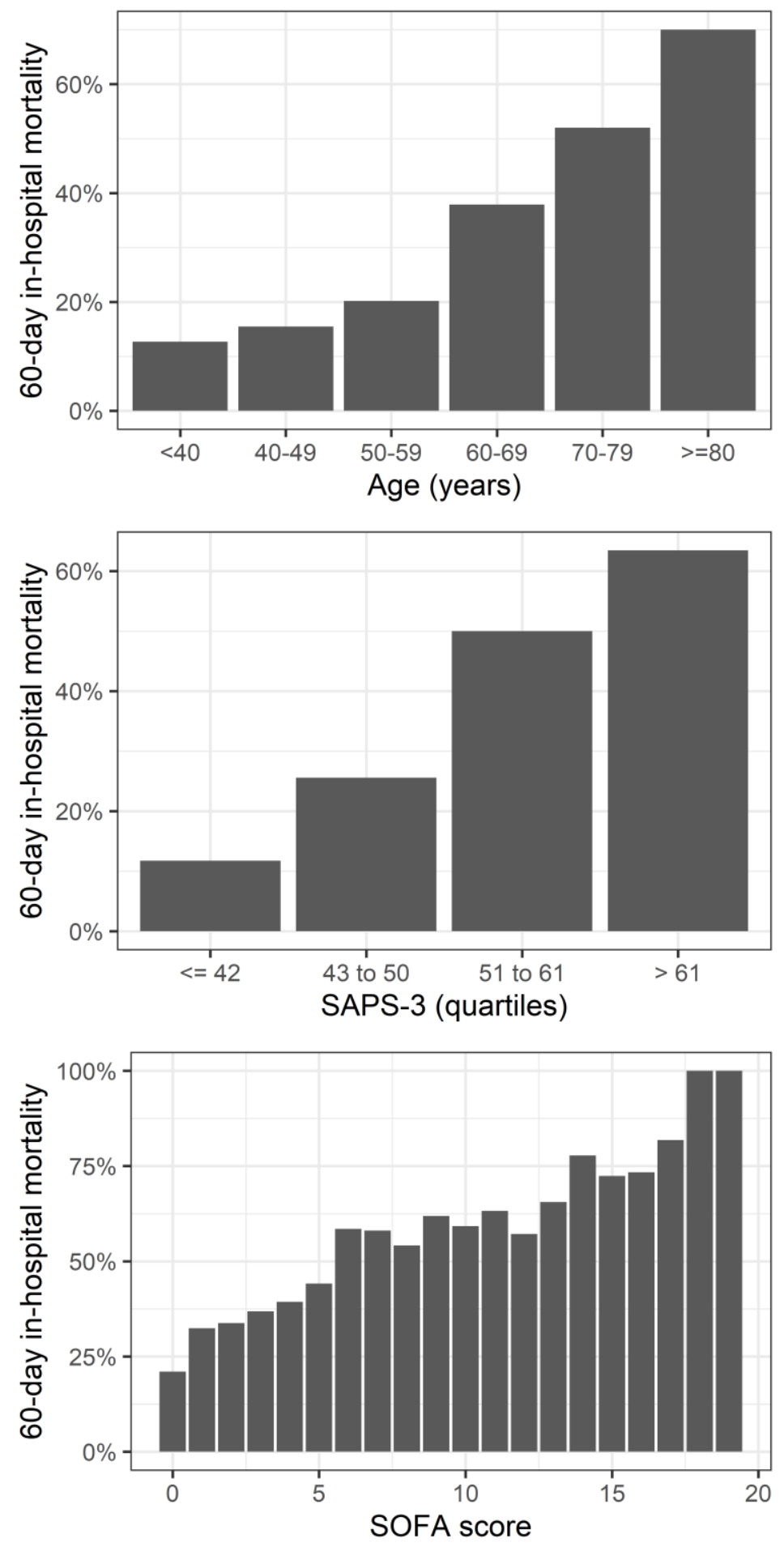

Figure A12.14 - Distribution of 60-day in-hospital mortality per age, SOFA, and SAPS-3 from patients that underwent advanced respiratory support $(n=4,188)$ 

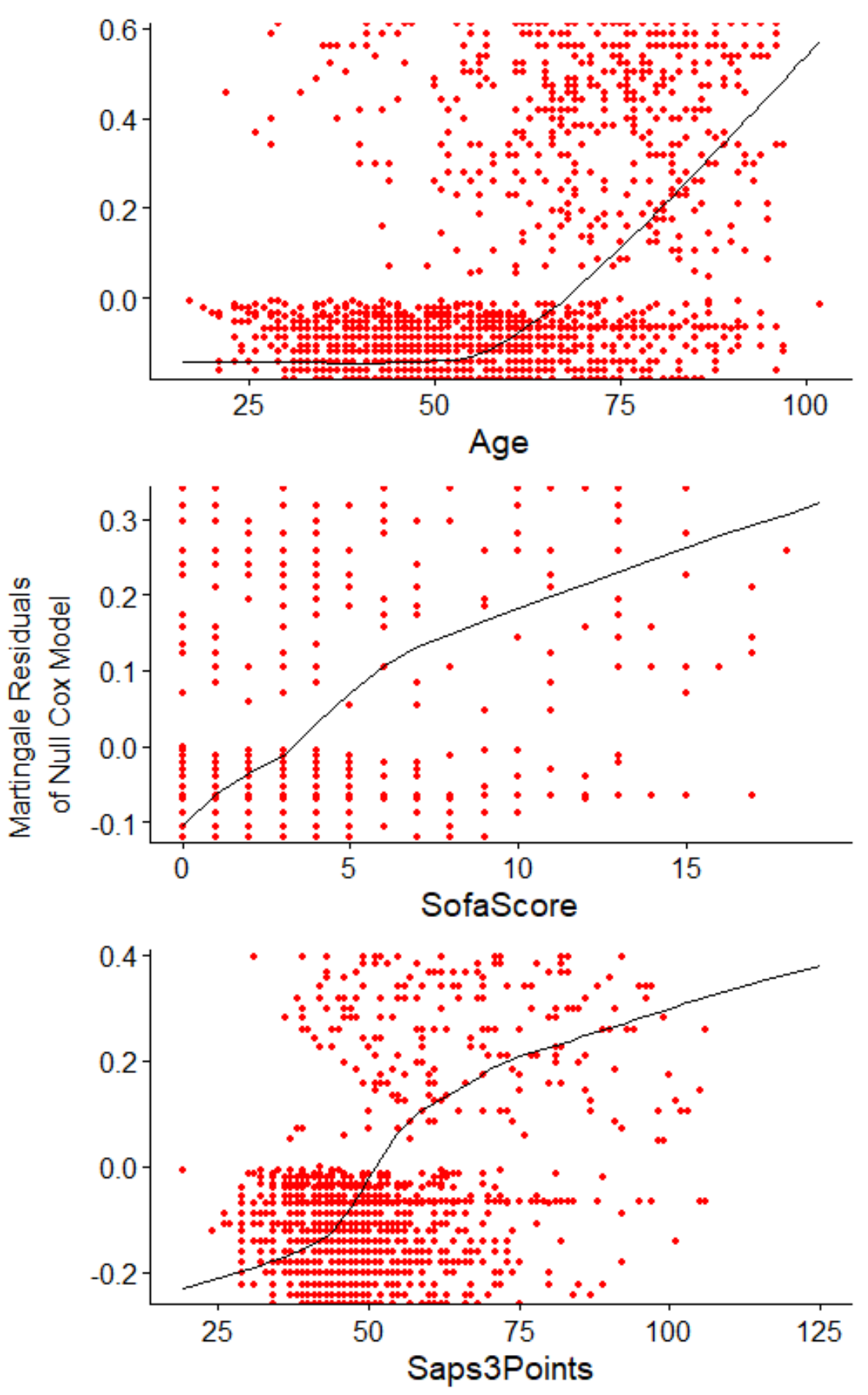

Figure A12.15 - Martingale residuals for Age, SOFA, and SAPS-3

Martingale residuals' plots were obtained using ggcoxfunction() from survminer package 
Table A12.40 - Clinical characteristics and outcomes of critically ill COVID-19 patients that underwent advanced respiratory support $(n=4,188)$, stratified by the first support used (IMV invasive mechanical ventilation, NIRS - Noninvasive respiratory)

\begin{tabular}{|c|c|c|c|}
\hline Characteristics & $\begin{array}{c}\text { Total } \\
{[n=4,188]}\end{array}$ & $\begin{array}{c}\text { IMV first } \\
{[n=1,765]}\end{array}$ & $\begin{array}{c}\text { NIRS first } \\
{[n=2,423]}\end{array}$ \\
\hline Age, Median (IQR) & $63(49,76)$ & $68(54,80)$ & $58(46,71)$ \\
\hline$<40$, No. $(\%)$ & $482(12 \%)$ & $131(7.4 \%)$ & $351(14 \%)$ \\
\hline $40-49$ & $601(14 \%)$ & $189(11 \%)$ & $412(17 \%)$ \\
\hline $50-59$ & $779(19 \%)$ & $260(15 \%)$ & $519(21 \%)$ \\
\hline $60-69$ & $840(20 \%)$ & $343(19 \%)$ & $497(21 \%)$ \\
\hline 70-79 & $696(17 \%)$ & $391(22 \%)$ & $305(13 \%)$ \\
\hline$\geq 80$ & $790(19 \%)$ & $451(26 \%)$ & $339(14 \%)$ \\
\hline \multicolumn{4}{|l|}{ Sex, No. (\%) } \\
\hline Female & $1,516(36 \%)$ & $665(38 \%)$ & $851(35 \%)$ \\
\hline Male & $2,672(64 \%)$ & $1,100(62 \%)$ & $1,572(65 \%)$ \\
\hline Admissions from emergency department, No. (\%) & $2,848(68 \%)$ & $1,023(58 \%)$ & $1,825(75 \%)$ \\
\hline \multicolumn{4}{|c|}{ Modified Frailty Index (MFI), No. (\%) } \\
\hline Non-frail $(M F I=0)$ & $1,164(28 \%)$ & $348(20 \%)$ & $816(34 \%)$ \\
\hline Pre-frail (MFI = 1-2) & $2,128(51 \%)$ & $937(53 \%)$ & $1,191(49 \%)$ \\
\hline Frail $(M F I>=3)$ & $896(21 \%)$ & $480(27 \%)$ & $416(17 \%)$ \\
\hline SAPS-3, Median (IQR) & $50(42,61)$ & $55(46,67)$ & $46(39,56)$ \\
\hline SOFA, Median (IQR) & $2.0(0.0,5.0)$ & $4.0(1.0,8.0)$ & $1.0(0.0,4.0)$ \\
\hline Hypertension, No. (\%) & $2,310(55 \%)$ & $1,089(62 \%)$ & $1,221(50 \%)$ \\
\hline Diabetes, No. (\%) & $1,421(34 \%)$ & $664(38 \%)$ & 757 (31\%) \\
\hline Cardiovascular disease, №. (\%) & $766(18 \%)$ & $377(21 \%)$ & $389(16 \%)$ \\
\hline Obesity, No. (\%) & $543(13 \%)$ & $188(11 \%)$ & $355(15 \%)$ \\
\hline COPD or Asthma, No. (\%) & $419(10 \%)$ & $193(11 \%)$ & $226(9.3 \%)$ \\
\hline $\mathrm{PaO2} / \mathrm{FiO2}[\mathrm{n}=4,649]$ & $2,310(55 \%)$ & $1,089(62 \%)$ & $1,221(50 \%)$ \\
\hline Normal (> 300), No. (\%) & $431(22 \%)$ & $217(21 \%)$ & $214(23 \%)$ \\
\hline Mild (201-300) & $385(20 \%)$ & $205(20 \%)$ & $180(19 \%)$ \\
\hline Moderate (101-200) & $621(32 \%)$ & $352(34 \%)$ & $269(29 \%)$ \\
\hline Severe $(\leq 100)$ & $526(27 \%)$ & $255(25 \%)$ & $271(29 \%)$ \\
\hline \multicolumn{4}{|l|}{ Advanced respiratory support, №. (\%) } \\
\hline Only NIRS, No. (\%) & $1,558(37 \%)$ & $0(0 \%)$ & $1,558(64 \%)$ \\
\hline NIRS failure, No. (\%) & $865(21 \%)$ & $0(0 \%)$ & $865(36 \%)$ \\
\hline Only IMV, No. (\%) & $1,765(42 \%)$ & $1,765(100 \%)$ & $0(0 \%)$ \\
\hline Vasopressor (first 24h), No. (\%) & $1,065(25 \%)$ & $710(40 \%)$ & $355(15 \%)$ \\
\hline Renal Replacement Therapy (first 24h), No. (\%) & $201(4.8 \%)$ & $130(7.4 \%)$ & $71(2.9 \%)$ \\
\hline Vasopressor, No. (\%) & $1,890(45 \%)$ & $1,158(66 \%)$ & $732(30 \%)$ \\
\hline Renal Replacement Therapy, №. (\%) & $896(21 \%)$ & $594(34 \%)$ & $302(12 \%)$ \\
\hline \multicolumn{4}{|l|}{ Length-of-stay (LOS), Median (IQR) } \\
\hline $\mathrm{ICU}[\mathrm{n}=4,185]$ & $12(7,22)$ & $16(9,27)$ & $10(6,17)$ \\
\hline Hospital $[n=4,160]$ & $17(10,30)$ & $22(12,38)$ & $14(9,24)$ \\
\hline \multicolumn{4}{|l|}{ Hospitalizations with LOS > 7 days, No. (\%) } \\
\hline $\operatorname{ICU}[\mathrm{n}=4,185]$ & $3,011(72 \%)$ & $1,436(81 \%)$ & $1,575(65 \%)$ \\
\hline Hospital $[n=4,160]$ & $3,496(84 \%)$ & $1,526(87 \%)$ & $1,970(82 \%)$ \\
\hline Period 1 (February 27th to April 25th), No. (\%) & $727(17 \%)$ & $545(31 \%)$ & $182(7.5 \%)$ \\
\hline Period 2 (April 26th to June 6th) & $1,264(30 \%)$ & $697(39 \%)$ & $567(23 \%)$ \\
\hline Period 3 (June 7th to August 10th) & $1,058(25 \%)$ & $286(16 \%)$ & $772(32 \%)$ \\
\hline Period 4 (August 11th to October 28th) & $1,139(27 \%)$ & $237(13 \%)$ & $902(37 \%)$ \\
\hline 60-day in-hospital deaths, No. (\%) & $1,544(37 \%)$ & $1,028(58 \%)$ & $516(21 \%)$ \\
\hline
\end{tabular}

IQR - Interquartile Range; SAPS - Simplified Acute Physiology Score; SOFA - Sequential Organ Failure Assessment; COPD - Chronic Obstructive Pulmonary Disease; NIRS - Noninvasive Respiratory Support; NPPV - Noninvasive Positive Pressure Ventilation; HFNC - High-Flow Nasal Cannula; IMV - Invasive Mechanical Ventilation; ICU Intensive care unit 

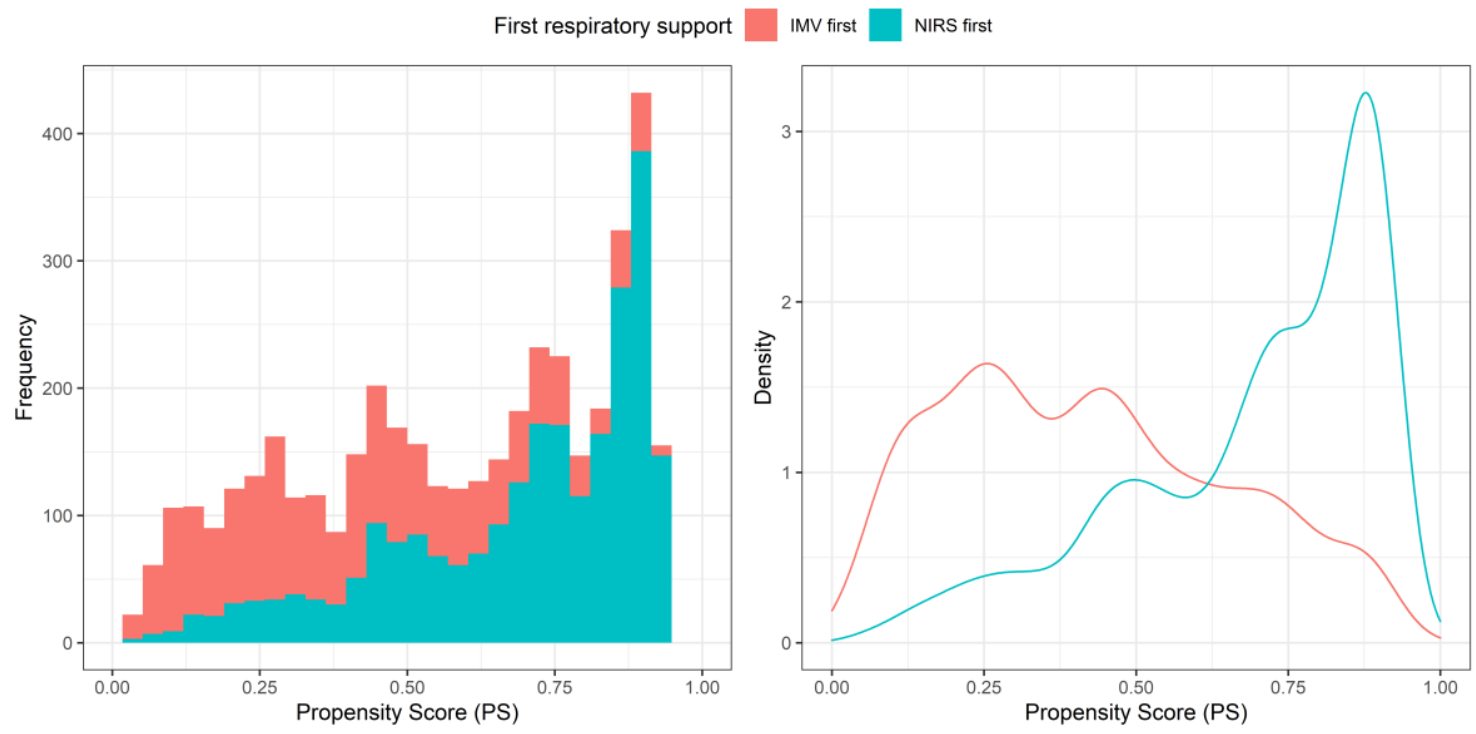

Figure A12.16 - Distribution of propensity scores among those treated initially with noninvasive respiratory support (NIRS first) and those treated initially with invasive mechanical ventilation (IMV first) 
Table A12.41 - Comparison of three multivariable logistic regression modelling approaches for propensity score estimation (patients that underwent advanced respiratory support, $n=4,188$ )

\begin{tabular}{|c|c|c|c|c|c|c|}
\hline \multirow[b]{2}{*}{ Variable } & \multicolumn{2}{|c|}{$\begin{array}{c}\text { All clinically relevant } \\
\text { variables }\end{array}$} & \multicolumn{2}{|c|}{$\begin{array}{l}\text { Without non-statistically } \\
\text { significant comorbidities }\end{array}$} & \multicolumn{2}{|c|}{$\begin{array}{c}\text { Without any non-statistically } \\
\text { significant variable }\end{array}$} \\
\hline & OR (95\% Cl) & $\mathbf{p}$ & OR $(95 \% \mathrm{Cl})$ & p & OR (95\% Cl) & $\mathbf{p}$ \\
\hline \multicolumn{7}{|l|}{ Sex } \\
\hline Female & Ref. & & Ref. & & Ref. & \\
\hline Male & $1.01(0.87-1.18)$ & 0.855 & $1.01(0.87-1.18)$ & 0.885 & $1.01(0.87-1.18)$ & 0.886 \\
\hline \multicolumn{7}{|l|}{ Age } \\
\hline$<40$ & Ref. & & Ref. & & Ref. & \\
\hline $40-49$ & $0.93(0.68-1.25)$ & 0.62 & $0.92(0.68-1.25)$ & 0.602 & $0.92(0.68-1.25)$ & 0.606 \\
\hline $50-59$ & $0.93(0.69-1.24)$ & 0.607 & $0.91(0.68-1.22)$ & 0.54 & $0.91(0.68-1.22)$ & 0.545 \\
\hline $60-69$ & $0.67(0.5-0.9)$ & 0.009 & $0.66(0.49-0.88)$ & 0.005 & $0.66(0.49-0.88)$ & 0.005 \\
\hline $70-79$ & $0.37(0.27-0.5)$ & $<0.001$ & $0.36(0.26-0.48)$ & $<0.001$ & $0.36(0.26-0.48)$ & $<0.001$ \\
\hline$\geq 80$ & $0.38(0.28-0.52)$ & $<0.001$ & $0.37(0.27-0.5)$ & $<0.001$ & $0.37(0.27-0.5)$ & $<0.001$ \\
\hline \multicolumn{7}{|l|}{ Modified Frailty Index } \\
\hline Non-frail $(M F I=0)$ & Ref. & & Ref. & & Ref. & \\
\hline Pre-frail (MFI = 1-2) & $0.75(0.59-0.97)$ & 0.028 & $0.8(0.67-0.97)$ & 0.024 & $0.8(0.67-0.97)$ & 0.024 \\
\hline Frail $(\mathrm{MFI}>=3$ ) & $0.77(0.53-1.11)$ & 0.163 & $0.83(0.64-1.07)$ & 0.144 & $0.83(0.64-1.07)$ & 0.148 \\
\hline SOFA & $0.95(0.93-0.98)$ & $<0.001$ & $0.95(0.93-0.98)$ & $<0.001$ & $0.96(0.93-0.98)$ & $<0.001$ \\
\hline $\begin{array}{l}\text { Admission from emergency } \\
\text { department }\end{array}$ & $1.98(1.7-2.31)$ & $<0.001$ & $1.98(1.7-2.31)$ & $<0.001$ & $1.98(1.7-2.3)$ & $<0.001$ \\
\hline Hypertension & $1.1(0.9-1.35)$ & 0.359 & & & & \\
\hline Diabetes & $0.96(0.8-1.15)$ & 0.664 & & & & \\
\hline Obesity & $1.16(0.93-1.46)$ & 0.197 & & & & \\
\hline COPD or Asthma & $1.04(0.81-1.33)$ & 0.78 & & & & \\
\hline Cardiovascular disease & $1.33(1.08-1.63)$ & 0.008 & $1.32(1.08-1.63)$ & 0.008 & $1.32(1.08-1.63)$ & 0.008 \\
\hline Vasopressor (first 24h) & $0.45(0.36-0.56)$ & $<0.001$ & $0.45(0.36-0.56)$ & $<0.001$ & $0.45(0.36-0.56)$ & $<0.001$ \\
\hline $\begin{array}{l}\text { Renal Replacement Therapy } \\
\text { (first 24h) }\end{array}$ & $1.08(0.75-1.55)$ & 0.658 & $1.1(0.76-1.57)$ & 0.619 & & \\
\hline $\begin{array}{l}\text { Akaike Information Criteria } \\
\text { (AIC) }\end{array}$ & \multicolumn{2}{|l|}{4506.90} & \multicolumn{2}{|l|}{4501.96} & \multicolumn{2}{|l|}{4500.21} \\
\hline $\begin{array}{l}\text { Bayesian Information Criteria } \\
\text { (BIC) }\end{array}$ & \multicolumn{2}{|l|}{4640.04} & \multicolumn{2}{|l|}{4609.74} & \multicolumn{2}{|l|}{4601.65} \\
\hline AUROC (95\% Cl) & \multicolumn{2}{|c|}{$0.804(0.793-0.19)$} & \multicolumn{2}{|c|}{$0.803(0.792-0.818)$} & \multicolumn{2}{|c|}{$0.803(0.792-0.818)$} \\
\hline Brier Score (95\% Cl) & \multicolumn{2}{|c|}{$0.177(0.170-0.182)$} & \multicolumn{2}{|c|}{$0.178(0.171-0.183)$} & \multicolumn{2}{|c|}{$0.78(0.171-0.183)$} \\
\hline
\end{tabular}

OR - Odds Ratio; SAPS-3 - Simplified Acute Physiology Score; SOFA- Sequential Organ Failure Assessment; COPD - Chronic Obstructive Pulmonary Disease; ICU - Intensive care unit; AUROC - Area under the receiving operating characteristic curve.

AUROC and Brier Score's respective 95\% confidence intervals were obtained using 1,000 resamples. 
Table A12.42 - Comparison full and reduced random-effects multivariable cox regression modelling approaches (patients that underwent advanced respiratory support, $n=4,188$ )

\begin{tabular}{|c|c|c|c|c|}
\hline \multirow[b]{2}{*}{ Variable } & \multicolumn{2}{|c|}{ All clinically relevant variables } & \multicolumn{2}{|l|}{$\begin{array}{c}\text { Without non- } \\
\text { statistically } \\
\text { significant }\end{array}$} \\
\hline & Hazard Ratio (95\% Cl) & $\mathbf{p}$ & $\begin{array}{c}\text { Hazard Ratio (95\% } \\
\mathrm{Cl})\end{array}$ & $\mathbf{p}$ \\
\hline \multicolumn{5}{|l|}{ Age } \\
\hline$<40$ & Ref. & & Ref. & \\
\hline $40-49$ & $1.11(0.90-1.37)$ & 0.319 & $1.01(0.89-1.35)$ & 0.389 \\
\hline $50-59$ & $1.09(0.89-1.33)$ & 0.399 & $1.07(0.87-1.30)$ & 0.524 \\
\hline $60-69$ & $1.47(1.20-1.8)$ & $<0.001$ & $1.43(1.17-1.75)$ & $<0.001$ \\
\hline $70-79$ & $1.71(1.38-2.10)$ & $<0.001$ & $1.66(1.35-2.05)$ & $<0.001$ \\
\hline$\geq 80$ & $2.75(2.21-3.41)$ & $<0.001$ & $2.64(2.14-3.27)$ & $<0.001$ \\
\hline \multicolumn{5}{|l|}{ Sex } \\
\hline Female & Ref. & & Ref. & \\
\hline Male & $1.00(0.93-1.08)$ & 0.948 & $1.00(0.93-1.08)$ & 0.989 \\
\hline \multicolumn{5}{|l|}{ Modified Frailty Index } \\
\hline Non-frail $(\mathrm{MFI}=0)$ & Ref. & & Ref. & \\
\hline Pre-frail (MFI = 1-2) & $1.24(1.09-1.42)$ & 0.001 & $1.26(1.11-1.43)$ & $<0.001$ \\
\hline Frail (MFI >= 3) & $1.38(1.15-1.64)$ & $<0.001$ & $1.43(1.22-1.67)$ & $<0.001$ \\
\hline SOFA & $1.04(1.02-1.05)$ & $<0.001$ & $1.04(1.02-1.05)$ & $<0.001$ \\
\hline \multicolumn{5}{|l|}{ SAPS-3 (quartiles) } \\
\hline$\leq 42$ & Ref, & & Ref. & \\
\hline $43-50$ & $1.35(1.16-1.57)$ & $<0.001$ & $1.34(1.15-1.56)$ & $<0.001$ \\
\hline $51-61$ & $1.64(1.41-1.92)$ & $<0.001$ & $1.63(1.40-1.91)$ & $<0.001$ \\
\hline$>61$ & $1.47(1.22-1.77)$ & $<0.001$ & $1.46(1.21-1.76)$ & $<0.001$ \\
\hline Admission from emergency department & $1.38(1.27-1.49)$ & $<0.001$ & $1.37(1.27-1.49)$ & $<0.001$ \\
\hline Hypertension & $0.81(0.73-0.88)$ & $<0.001$ & $0.81(0.74-0.89)$ & $<0.001$ \\
\hline Diabetes & $1.03(0.94-1.12)$ & 0.546 & & \\
\hline Obesity & $1.11(0.99-1.24)$ & 0.085 & & \\
\hline COPD or Asthma & $1.07(0.96-1.20)$ & 0.206 & & \\
\hline Cardiovascular disease & $1.12(1.02-1.22)$ & 0.015 & $1.11(1.02-1.22)$ & 0.021 \\
\hline Vasopressor & $1.30(1.19-1.43)$ & $<0.001$ & $1.30(1.19-1.43)$ & $<0.001$ \\
\hline Renal Replacement Therapy & $1.44(1.33-1.56)$ & $<0.001$ & $1.45(1.34-1.57)$ & $<0.001$ \\
\hline \multicolumn{5}{|l|}{ First respiratory support } \\
\hline Noninvasive Respiratory Support & $0.59(0.54-0.65)$ & $<0.001$ & $0.59(0.55-0.65)$ & $<0.001$ \\
\hline Invasive Mechanical Ventilation & Ref. & & Ref. & \\
\hline SD Random Intercept (Hospital) & 0.50 & & 0.50 & \\
\hline Akaike Information Criteria (AIC) & 1623.63 & & 1624.74 & \\
\hline Bayesian Information Criteria (BIC) & 1490.01 & & 1507.21 & \\
\hline
\end{tabular}

HR - Hazard Ratio; SAPS-3 - Simplified Acute Physiology Score; SOFA- Sequential Organ Failure Assessment; COPD - Chronic Obstructive Pulmonary Disease; ICU - Intensive care unit 


\subsection{6}

\section{Appendix A6.6 - Sensitivity Analyses}

Table A12.43 - Random-effects multivariable cox regression model with SMR-weighting from propensity scores and adjusted by the time periods defined using the estimated breakpoints (Sensitivity analysis)

\begin{tabular}{|c|c|c|}
\hline \multirow[b]{2}{*}{ Variable } & \multicolumn{2}{|c|}{$\begin{array}{l}\text { Advanced respiratory support } \\
\qquad(\mathrm{n}=4,188)\end{array}$} \\
\hline & Hazard Ratio (95\% Cl) & $\mathbf{p}$ \\
\hline \multicolumn{3}{|l|}{ Age } \\
\hline$<40$ & Ref. & \\
\hline $40-49$ & $1.261(0.96-1.655)$ & 0.095 \\
\hline $50-59$ & $1.111(0.857-1.44)$ & 0.426 \\
\hline $60-69$ & $1.736(1.334-2.261)$ & $<0.001$ \\
\hline $70-79$ & $1.974(1.488-2.62)$ & $<0.001$ \\
\hline$\geq 80$ & $3.263(2.439-4.366)$ & $<0.001$ \\
\hline \multicolumn{3}{|l|}{ Sex } \\
\hline Female & Ref. & \\
\hline Male & $0.928(0.834-1.032)$ & 0.167 \\
\hline \multicolumn{3}{|l|}{ Modified Frailty Index } \\
\hline Non-frail $(\mathrm{MFI}=0)$ & Ref. & \\
\hline Pre-frail (MFI = 1-2) & $1.211(1.011-1.451)$ & 0.038 \\
\hline Frail $(\mathrm{MFI}>=3)$ & $1.231(0.953-1.589)$ & 0.111 \\
\hline SOFA & $1.054(1.034-1.075)$ & $<0.001$ \\
\hline \multicolumn{3}{|l|}{ SAPS-3 (quartiles) } \\
\hline$\leq 42$ & Ref. & \\
\hline $43-50$ & $1.369(1.126-1.665)$ & 0.002 \\
\hline $51-61$ & $1.539(1.25-1.895)$ & $<0.001$ \\
\hline$>61$ & $1.393(1.075-1.806)$ & 0.012 \\
\hline Admission from emergency department & $1.236(1.096-1.393)$ & $<0.001$ \\
\hline Hypertension & $0.85(0.742-0.973)$ & 0.018 \\
\hline Diabetes & $1.047(0.925-1.184)$ & 0.469 \\
\hline Obesity & $1.172(0.998-1.376)$ & 0.053 \\
\hline COPD or Asthma & $1.047(0.888-1.233)$ & 0.585 \\
\hline Cardiovascular disease & $1.157(1.013-1.321)$ & 0.031 \\
\hline Vasopressor & $1.338(1.184-1.513)$ & $<0.001$ \\
\hline Renal Replacement Therapy & $1.625(1.448-1.825)$ & $<0.001$ \\
\hline \multicolumn{3}{|l|}{ Initial respiratory support } \\
\hline Noninvasive Respiratory Support first & $0.637(0.563-0.721)$ & $<0.001$ \\
\hline Invasive Mechanical Ventilation first & Ref. & \\
\hline SD Random Intercept (Hospital) & 0.51 & \\
\hline
\end{tabular}


Table A12.44 - Random-effects multivariable cox regression model with inverse probability treatment weighting (IPTW) using trimmed propensity scores (percentile 95\%) and adjusted by the time periods defined using the estimated breakpoints (Sensitivity analysis)

\begin{tabular}{|c|c|c|}
\hline \multirow[b]{2}{*}{ Variable } & \multicolumn{2}{|c|}{$\begin{array}{l}\text { Advanced respiratory support } \\
(n=3,982)\end{array}$} \\
\hline & Hazard Ratio (95\% Cl) & $\mathbf{p}$ \\
\hline \multicolumn{3}{|l|}{ Age } \\
\hline$<40$ & Ref. & \\
\hline $40-49$ & $1.249(1.004-1.554)$ & 0.046 \\
\hline $50-59$ & $1.197(0.972-1.475)$ & 0.091 \\
\hline $60-69$ & $1.56(1.265-1.925)$ & $<0.001$ \\
\hline $70-79$ & $1.833(1.475-2.277)$ & $<0.001$ \\
\hline$\geq 80$ & $2.961(2.369-3.701)$ & $<0.001$ \\
\hline \multicolumn{3}{|l|}{ Sex } \\
\hline Female & Ref. & \\
\hline Male & $1.017(0.944-1.095)$ & 0.661 \\
\hline \multicolumn{3}{|l|}{ Modified Frailty Index } \\
\hline Non-frail $(\mathrm{MFI}=0)$ & Ref. & \\
\hline Pre-frail (MFI = 1-2) & $1.221(1.07-1.394)$ & 0.003 \\
\hline Frail $(\mathrm{MFI}>=3)$ & $1.365(1.143-1.63)$ & $<0.001$ \\
\hline SOFA & $1.038(1.025-1.052)$ & $<0.001$ \\
\hline \multicolumn{3}{|l|}{ SAPS-3 (quartiles) } \\
\hline$\leq 42$ & Ref. & \\
\hline $43-50$ & $1.313(1.126-1.531)$ & $<0.001$ \\
\hline $51-61$ & $1.588(1.357-1.858)$ & $<0.001$ \\
\hline$>61$ & $1.419(1.178-1.71)$ & $<0.001$ \\
\hline Admission from emergency department & $1.39(1.283-1.506)$ & $<0.001$ \\
\hline Hypertension & $0.81(0.737-0.889)$ & $<0.001$ \\
\hline Diabetes & $1.021(0.939-1.11)$ & 0.629 \\
\hline Obesity & $1.077(0.957-1.212)$ & 0.217 \\
\hline COPD or Asthma & $1.074(0.963-1.198)$ & 0.202 \\
\hline Cardiovascular disease & $1.126(1.029-1.233)$ & 0.01 \\
\hline Vasopressor & $1.245(1.139-1.362)$ & $<0.001$ \\
\hline Renal Replacement Therapy & $1.467(1.355-1.588)$ & $<0.001$ \\
\hline \multicolumn{3}{|l|}{ Initial respiratory support } \\
\hline Noninvasive Respiratory Support first & $0.589(0.54-0.641)$ & $<0.001$ \\
\hline Invasive Mechanical Ventilation first & Ref. & \\
\hline SD Random Intercept (Hospital) & 0.49 & \\
\hline
\end{tabular}


Table A 12.45 -Comparison of three multivariable logistic regression modelling approaches for propensity score estimation in the subset of patients that presented $\mathrm{PaO}_{2} / \mathrm{FiO}_{2}$ values $(\mathrm{n}=$ 1,963 ) and adjusted by the time periods defined using the estimated breakpoints (Sensitivity analysis)

\begin{tabular}{|c|c|c|c|c|c|c|}
\hline \multirow[b]{2}{*}{ Variable } & \multicolumn{2}{|c|}{ All clinically relevant variables } & \multicolumn{2}{|c|}{$\begin{array}{l}\text { Without non-statistically } \\
\text { significant comorbidities }\end{array}$} & \multicolumn{2}{|c|}{$\begin{array}{c}\text { Without any non-statistically } \\
\text { significant variable }\end{array}$} \\
\hline & OR (95\% Cl) & p & OR (95\% Cl) & $\mathbf{p}$ & OR $(95 \% \mathrm{Cl})$ & p \\
\hline Intercept & $0.83(0.51-1.34)$ & 0.451 & $0.82(0.51-1.32)$ & 0.417 & $0.85(0.54-1.32)$ & 0.462 \\
\hline \multicolumn{7}{|l|}{ Sex } \\
\hline Female & Ref. & & Ref. & & Ref. & \\
\hline Male & $0.92(0.75-1.13)$ & 0.425 & $0.94(0.76-1.15)$ & 0.544 & $0.94(0.76-1.15)$ & 0.529 \\
\hline \multicolumn{7}{|l|}{ Age } \\
\hline$<40$ & Ref. & & Ref. & & Ref. & \\
\hline $40-49$ & $1.06(0.67-1.66)$ & 0.811 & $1.04(0.66-1.64)$ & 0.863 & $1.05(0.67-1.64)$ & 0.847 \\
\hline $50-59$ & $1.06(0.68-1.64)$ & 0.81 & $1.05(0.68-1.64)$ & 0.816 & $1.06(0.68-1.64)$ & 0.802 \\
\hline $60-69$ & $0.97(0.62-1.51)$ & 0.898 & $0.97(0.62-1.5)$ & 0.889 & $0.96(0.62-1.48)$ & 0.841 \\
\hline $70-79$ & $0.5(0.31-0.79)$ & 0.003 & $0.51(0.32-0.8)$ & 0.004 & $0.5(0.32-0.78)$ & 0.003 \\
\hline$\geq 80$ & $0.51(0.32-0.8)$ & 0.004 & $0.53(0.33-0.84)$ & 0.007 & $0.52(0.33-0.82)$ & 0.005 \\
\hline \multicolumn{7}{|l|}{ Modified Frailty Index } \\
\hline Non-frail (MFI = 0) & Ref. & & Ref. & & Ref. & \\
\hline Pre-frail (MFI = 1-2) & $0.93(0.65-1.33)$ & 0.697 & $0.91(0.69-1.2)$ & 0.502 & $0.91(0.69-1.21)$ & 0.515 \\
\hline Frail (MFI >=3) & $1.28(0.77-2.11)$ & 0.342 & $1.23(0.87-1.72)$ & 0.239 & $1.23(0.88-1.72)$ & 0.237 \\
\hline $\begin{array}{l}\text { SOFA } \\
\text { Admission from }\end{array}$ & $0.96(0.93-1)$ & 0.041 & $0.97(0.93-1)$ & 0.053 & $0.98(0.95-1.01)$ & 0.136 \\
\hline $\begin{array}{l}\text { emergency } \\
\text { department }\end{array}$ & $1.6(1.3-1.97)$ & $<0.001$ & $1.58(1.29-1.95)$ & $<0.001$ & $1.57(1.28-1.92)$ & $<0.001$ \\
\hline Hypertension & $1.05(0.79-1.38)$ & 0.749 & & & & \\
\hline Diabetes & $0.84(0.65-1.08)$ & 0.18 & & & & \\
\hline Obesity & $1.76(1.3-2.38)$ & $<0.001$ & $1.72(1.28-2.32)$ & $<0.001$ & $1.72(1.28-2.32)$ & $<0.001$ \\
\hline COPD or Asthma & $0.83(0.6-1.15)$ & 0.26 & & & & \\
\hline $\begin{array}{l}\text { Cardiovascular } \\
\text { disease }\end{array}$ & $1.29(0.98-1.7)$ & 0.072 & & & & \\
\hline $\begin{array}{l}\text { Vasopressor (first } \\
\text { 24h) }\end{array}$ & $0.53(0.41-0.7)$ & $<0.001$ & $0.54(0.41-0.71)$ & $<0.001$ & $0.53(0.4-0.7)$ & $<0.001$ \\
\hline $\begin{array}{l}\text { Renal Replacement } \\
\text { Therapy (first 24h) }\end{array}$ & $1.11(0.73-1.7)$ & 0.616 & $1.14(0.74-1.73)$ & 0.553 & & \\
\hline \multicolumn{7}{|l|}{$\mathrm{PaO2} / \mathrm{FiO} 2$} \\
\hline Normal (> 300) & Ref. & & Ref. & & & \\
\hline Mild (201-300) & $0.94(0.69-1.28)$ & 0.696 & $0.95(0.69-1.29)$ & 0.722 & & \\
\hline Moderate (101-200) & $1.01(0.75-1.35)$ & 0.956 & $1.01(0.76-1.35)$ & 0.938 & & \\
\hline Severe $(\leq 100)$ & $1.29(0.95-1.75)$ & 0.107 & $1.28(0.94-1.74)$ & 0.114 & & \\
\hline $\begin{array}{l}\text { Akaike Information } \\
\text { Criteria (AIC) }\end{array}$ & 2340.83 & & 2339.65 & & 2336.70 & \\
\hline $\begin{array}{l}\text { Bayesian Information } \\
\text { Criteria (BIC) }\end{array}$ & 2474.80 & & 2451.30 & & 2426.02 & \\
\hline AUROC $(95 \% \mathrm{Cl})$ & $0.76(0.747-0.787)$ & & $0.758(0.743-0.78$ & & $0.756(0.74-0.78)$ & \\
\hline Brier Score $(95 \% \mathrm{Cl})$ & $0.199(0.188-0.204)$ & & $0.2(0.189-0.205)$ & & $0.201(0.191-0.206)$ & \\
\hline
\end{tabular}

OR - Odds Ratio; SAPS-3 - Simplified Acute Physiology Score; SOFA- Sequential Organ Failure Assessment; COPD - Chronic Obstructive Pulmonary Disease; ICU - Intensive care unit; AUROC - Area under the receiving operating characteristic curve.

AUROC and Brier Score's respective 95\% confidence intervals were obtained using 1,000 resamples.

The chosen model was the one with Sex, Age, MFI, SOFA, Admission from emergency department, Obesity and Vasopressor (first 24h) as predictors (lowest AIC and BIC) 
Table A12.46 -Random-effects multivariable cox regression model with inverse probability treatment weighting (IPTW) for the subset of patients that presented $\mathrm{PaO}_{2} / \mathrm{FiO}_{2}(\mathrm{~N}=1,963)$ values and adjusted by the time periods defined using the estimated breakpoints (Sensitivity analysis)

\begin{tabular}{|c|c|c|c|c|}
\hline \multirow[b]{2}{*}{ Variable } & \multicolumn{2}{|c|}{ All clinically relevant variables } & \multicolumn{2}{|l|}{$\begin{array}{l}\text { Without non-statistically } \\
\text { significant comorbidities }\end{array}$} \\
\hline & Hazard Ratio (95\% Cl) & $\mathbf{p}$ & Hazard Ratio (95\% Cl) & $\mathbf{p}$ \\
\hline \multicolumn{5}{|c|}{ 年 } \\
\hline$<40$ & Ref. & & Ref. & \\
\hline $40-49$ & $0.93(0.686-1.26)$ & 0.639 & $0.929(0.69-1.26)$ & 0.637 \\
\hline $50-59$ & $0.761(0.562-1.029)$ & 0.076 & $0.758(0.56-1.02)$ & 0.071 \\
\hline $60-69$ & $1.127(0.839-1.513)$ & 0.427 & $1.121(0.84-1.5)$ & 0.447 \\
\hline $70-79$ & $1.16(0.858-1.569)$ & 0.335 & $1.159(0.86-1.56)$ & 0.337 \\
\hline$\geq 80$ & $1.879(1.382-2.555)$ & $<0.001$ & $1.872(1.38-2.54)$ & $<0.001$ \\
\hline \multicolumn{5}{|l|}{ Sex } \\
\hline Female & Ref. & & Ref. & \\
\hline Male & $0.932(0.841-1.033)$ & 0.181 & $0.933(0.84-1.03)$ & 0.185 \\
\hline \multicolumn{5}{|l|}{ Modified Frailty Index } \\
\hline Non-frail (MFI = 0) & Ref. & & Ref. & \\
\hline Pre-frail (MFI = 1-2) & $1.134(0.939-1.37)$ & 0.19 & $1.143(0.95-1.37)$ & 0.152 \\
\hline Frail $(\mathrm{MFI}>=3)$ & $1.39(1.086-1.779)$ & 0.009 & $1.424(1.15-1.77)$ & 0.001 \\
\hline $\begin{array}{l}\text { SOFA } \\
\text { SAPS-3 (quartiles) }\end{array}$ & $1.022(1.004-1.04)$ & 0.018 & $1.022(1-1.04)$ & 0.016 \\
\hline $\begin{array}{l}\leq 42 \\
43-50 \\
51-61 \\
>61\end{array}$ & $\begin{array}{c}\text { Ref, } \\
1.565(1.181-2.074) \\
2.145(1.633-2.819) \\
1.97(1.463-2.654)\end{array}$ & $\begin{array}{c}0.002 \\
<0.001 \\
<0.001\end{array}$ & $\begin{array}{c}\text { Ref. } \\
1.555(1.17-2.06) \\
2.132(1.62-2.8) \\
1.956(1.45-2.63)\end{array}$ & $\begin{array}{c}0.002 \\
<0.001 \\
<0.001\end{array}$ \\
\hline $\begin{array}{l}\text { Admission from emergency } \\
\text { department }\end{array}$ & $1.492(1.337-1.665)$ & $<0.001$ & $1.491(1.34-1.66)$ & $<0.001$ \\
\hline Hypertension & $0.755(0.667-0.856)$ & $<0.001$ & $0.754(0.67-0.85)$ & $<0.001$ \\
\hline Diabetes & $1.002(0.892-1.124)$ & 0.977 & & \\
\hline Obesity & $1.046(0.895-1.222)$ & 0.573 & & \\
\hline COPD or Asthma & $1.167(1.01-1.349)$ & 0.037 & $1.165(1.01-1.34)$ & 0.037 \\
\hline Cardiovascular disease & $1.051(0.928-1.189)$ & 0.433 & & \\
\hline Vasopressor & $1.397(1.224-1.596)$ & $<0.001$ & $1.399(1.23-1.6)$ & $<0.001$ \\
\hline $\begin{array}{l}\text { Renal Replacement Therapy } \\
\text { PaO2/FiO2 }\end{array}$ & $1.391(1.252-1.547)$ & $<0.001$ & $1.392(1.25-1.55)$ & $<0.001$ \\
\hline Normal $(>300)$ & Ref. & & Ref. & \\
\hline Mild (201-300) & $1.12(0.95-1.32)$ & 0.178 & $1.124(0.95-1.32)$ & 0.162 \\
\hline Moderate (101-200) & $1.099(0.943-1.282)$ & 0.228 & $1.099(0.94-1.28)$ & 0.226 \\
\hline Severe $(\leq 100)$ & $1.204(1.008-1.439)$ & 0.041 & $1.204(1.01-1.44)$ & 0.041 \\
\hline \multicolumn{5}{|l|}{ Initial respiratory support } \\
\hline $\begin{array}{l}\text { Noninvasive Respiratory } \\
\text { Support first }\end{array}$ & $0.564(0.503-0.632)$ & $<0.001$ & $0.564(0.5-0.63)$ & $<0.001$ \\
\hline $\begin{array}{l}\text { Invasive Mechanical } \\
\text { Ventilation first }\end{array}$ & Ref. & & Ref. & \\
\hline $\begin{array}{l}\text { SD Random Intercept } \\
\text { (Hospital) }\end{array}$ & 0.54 & & 0.54 & \\
\hline $\begin{array}{l}\text { Akaike Information Criteria } \\
\text { (AIC) }\end{array}$ & 812.32 & & 817.40 & \\
\hline $\begin{array}{l}\text { Bayesian Information } \\
\text { Criteria (BIC) }\end{array}$ & 678.36 & & 697.79 & \\
\hline
\end{tabular}

HR - Hazard Ratio; SAPS-3 - Simplified Acute Physiology Score; SOFA- Sequential Organ Failure Assessment; COPD - Chronic Obstructive Pulmonary Disease; ICU - Intensive care unit

The chosen model was the one with lowest AIC and BIC (all clinically relevant variables):

Fixed effects: Sex, Age, MFI, SOFA, SAPS-3, Admission from emergency, hypertension, diabetes, obesity, COPD or asthma, Cardiovascular disease, Vasopressor, Rental Replacement Therapy, $\mathrm{PaO} 2 / \mathrm{FiO} 2$ at admission, and Initial respiratory support

Random intercept: Hospital 
Table A12.47 -Missing pattern of $\mathrm{PaO} 2 / \mathrm{FiO} 2$ values

\begin{tabular}{|c|c|c|c|c|}
\hline \multirow[b]{2}{*}{ Variable } & \multicolumn{2}{|c|}{$\begin{array}{l}\text { All admissions } \\
(n=13,301)\end{array}$} & \multicolumn{2}{|c|}{$\begin{array}{l}\text { Advanced respiratory support } \\
\qquad(n=4,188)\end{array}$} \\
\hline & $\begin{array}{c}\text { Not Missing } \\
(\mathrm{n}=8652)\end{array}$ & $\begin{array}{l}\text { Missing } \\
(n=4649)\end{array}$ & $\begin{array}{c}\text { Not Missing } \\
(n=2225)\end{array}$ & $\begin{array}{c}\text { Missing } \\
(n=1963)\end{array}$ \\
\hline Age, median (IQR) & $51(40,66)$ & $59(46,73)$ & $59(46,73)$ & $66(53,78)$ \\
\hline$<40$ & $2129(25 \%)$ & $703(15 \%)$ & $324(15 \%)$ & $158(8.0 \%)$ \\
\hline $40-49$ & $1868(22 \%)$ & $768(17 \%)$ & $359(16 \%)$ & $242(12 \%)$ \\
\hline $50-59$ & $1691(20 \%)$ & $892(19 \%)$ & $460(21 \%)$ & $319(16 \%)$ \\
\hline $60-69$ & $1262(15 \%)$ & $826(18 \%)$ & $419(19 \%)$ & $421(21 \%)$ \\
\hline $70-79$ & $826(9.5 \%)$ & $676(15 \%)$ & $315(14 \%)$ & $381(19 \%)$ \\
\hline$\geq 80$ & $876(10 \%)$ & $784(17 \%)$ & $348(16 \%)$ & $442(23 \%)$ \\
\hline \multicolumn{5}{|l|}{ Sex, No. (\%) } \\
\hline Female & $3646(42 \%)$ & 1903 (41\%) & $766(34 \%)$ & $750(38 \%)$ \\
\hline Male & $5006(58 \%)$ & $2746(59 \%)$ & $1459(66 \%)$ & $1213(62 \%)$ \\
\hline $\begin{array}{l}\text { Admission from emergency } \\
\text { department, No. (\%) }\end{array}$ & $7123(82 \%)$ & $3117(67 \%)$ & $1668(75 \%)$ & $1180(60 \%)$ \\
\hline \multicolumn{5}{|l|}{ Modified Frailty Index, №. (\%) } \\
\hline Non-frail $(M F I=0)$ & $4298(50 \%)$ & $1562(34 \%)$ & $766(34 \%)$ & $398(20 \%)$ \\
\hline Pre-frail (MFI = 1-2) & $3575(41 \%)$ & $2142(46 \%)$ & $1118(50 \%)$ & $1010(51 \%)$ \\
\hline Frail $(\mathrm{MFI}>=3)$ & $779(9.0 \%)$ & $945(20 \%)$ & $341(15 \%)$ & $555(28 \%)$ \\
\hline SAPS-3, median (IQR) & $41(37,47)$ & $47(39,57)$ & $45(39,54)$ & $57(47,70)$ \\
\hline SOFA, median (IQR) & $0(0,1)$ & $2(1,4)$ & $0(0,2)$ & $4(2,8)$ \\
\hline Hypertension, No. (\%) & $3313(38 \%)$ & $2356(51 \%)$ & $1109(50 \%)$ & $1201(61 \%)$ \\
\hline Diabetes, No. (\%) & $1838(21 \%)$ & $1369(29 \%)$ & 701 (32\%) & $720(37 \%)$ \\
\hline Cardiovascular disease, No. (\%) & $910(11 \%)$ & $744(16 \%)$ & $351(16 \%)$ & $415(21 \%)$ \\
\hline Immunosuppression, No. (\%) & $1091(13 \%)$ & $705(15 \%)$ & $343(15 \%)$ & $356(18 \%)$ \\
\hline Obesity, No. (\%) & $662(7.7 \%)$ & $467(10 \%)$ & $268(12 \%)$ & $275(14 \%)$ \\
\hline COPD or Asthma, No. (\%) & $538(6.2 \%)$ & $442(9.5 \%)$ & $189(8.5 \%)$ & $230(12 \%)$ \\
\hline \multicolumn{5}{|l|}{ Repiratory Support, No. (\%) } \\
\hline Oxygen Support & $6427(74 \%)$ & $2686(58 \%)$ & - & - \\
\hline Advanced respiratory support & $2225(26 \%)$ & $1963(42 \%)$ & $2225(100 \%)$ & $1963(100 \%)$ \\
\hline NIRS only & $1127(13 \%)$ & $431(9.3 \%)$ & $1127(51 \%)$ & $431(22 \%)$ \\
\hline NIRS failure & $362(4.2 \%)$ & $503(11 \%)$ & $362(16 \%)$ & $503(26 \%)$ \\
\hline IMV only & $736(8.5 \%)$ & $1029(22 \%)$ & $736(33 \%)$ & $1029(52 \%)$ \\
\hline Vasopressor (first 24h), No. (\%) & $341(3.9 \%)$ & $784(17 \%)$ & 307 (14\%) & 758 (39\%) \\
\hline $\begin{array}{l}\text { Renal Replacement Therapy (first } \\
\text { 24h), No. (\%) }\end{array}$ & $76(0.9 \%)$ & $165(3.5 \%)$ & $56(2.5 \%)$ & $145(7.4 \%)$ \\
\hline $\begin{array}{l}\text { Period } 1 \text { (February 27th to April } \\
\text { 25th), No, (\%) }\end{array}$ & $1313(15 \%)$ & $871(19 \%)$ & $322(14 \%)$ & $405(21 \%)$ \\
\hline Period 2 (April 26th to June 6th) & $2060(24 \%)$ & $1476(32 \%)$ & $596(27 \%)$ & $668(34 \%)$ \\
\hline Period 3 (June 7th to August 10th) & $2689(31 \%)$ & $1249(27 \%)$ & $629(28 \%)$ & $429(22 \%)$ \\
\hline $\begin{array}{l}\text { Period } 4 \text { (August } 11 \text { th to October } \\
\text { 28th) }\end{array}$ & $2590(30 \%)$ & $1053(23 \%)$ & $678(30 \%)$ & $461(23 \%)$ \\
\hline ICU Length-of-stay, median (IQR) & $4(2,8)$ & $7(3,15)$ & $11(6,19)$ & $15(8,24)$ \\
\hline $\begin{array}{l}\text { Hospital Length-of-stay, median } \\
\text { (IQR) }\end{array}$ & $7(5,13)$ & $11(6,21)$ & $14(9,25)$ & $20(11,34)$ \\
\hline $\begin{array}{l}\text { 60-day in-hospital mortality, No. } \\
(\%)\end{array}$ & $796(9.2 \%)$ & $989(21 \%)$ & $660(30 \%)$ & $884(45 \%)$ \\
\hline
\end{tabular}

IQR - Interquartile Range; SAPS - Simplified Acute Physiology Score; SOFA - Sequential Organ Failure Assessment; COPD - Chronic Obstructive Pulmonary Disease; IMV - Invasive Mechanical Ventilation; ICU - Intensive care unit 
Table A12.48 -Random-effects multivariable cox regression model with inverse probability weighting (IPTW) and multiple imputation by chained equations of $\mathrm{PaO}_{2} / \mathrm{FiO}_{2}$ values. The model was adjusted by the time periods defined using the estimated breakpoints

We used multiple imputation with chained equations (MICE) to impute values of $\mathrm{PaO}_{2} / \mathrm{FiO}_{2}$ using auxiliary variables: Age; Sex, Modified Frailty Index; Simplified Acute Physiology Score (SAPS-3); Sequential Organ Failure Assessment (SOFA); Indicator of admission from emergency department; Presence of comorbidities (Hypertension, Diabetes, Obesity, COPD or Asthma; and Cardiovascular disease); Use of Non-invasive Respiratory Support [indicator] + Use of Invasive mechanical ventilation [indicator], 60-day in-hospital outcome, hospital length of stay, and the Hospital. We generated 100 imputed datasets, following recommendations(MADLEY-DOWD et al., 2019) and combined the results using Rubin's rule. (RUBIN, 1987)

We considered inverse probability treatment weighting (IPTW) mortality model as previously defined in Appendix A8.1. In this mortality model, we considered Sex, Age, MFI, SOFA, SAPS-3, Admission from emergency, hypertension, diabetes, obesity, COPD or asthma, Cardiovascular disease, Vasopressor, Rental Replacement Therapy, $\mathrm{PaO} / \mathrm{FiO} 2$ at admission, and Initial respiratory support as fixed effects and the Hospital as the Random intercept.

\begin{tabular}{|c|c|c|}
\hline \multirow[b]{2}{*}{ Variable } & \multicolumn{2}{|c|}{$\begin{array}{c}\text { Advanced respiratory support } \\
(n=4,188)\end{array}$} \\
\hline & Hazard Ratio (95\% Cl) & $\mathbf{p}$ \\
\hline \multicolumn{3}{|l|}{ Age } \\
\hline$<40$ & Ref. & \\
\hline $40-49$ & $1.14(0.91-1.42)$ & 0.238 \\
\hline $50-59$ & $1.09(0.88-1.34)$ & 0.41 \\
\hline $60-69$ & $1.49(1.2-1.84)$ & $<0.001$ \\
\hline $70-79$ & $1.73(1.39-2.16)$ & $<0.001$ \\
\hline$\geq 80$ & $2.84(2.26-3.57)$ & $<0.001$ \\
\hline \multicolumn{3}{|l|}{ Sex } \\
\hline Female & Ref. & \\
\hline Male & $0.99(0.92-1.07)$ & 0.806 \\
\hline \multicolumn{3}{|l|}{ Modified Frailty Index } \\
\hline Non-frail $(\mathrm{MFI}=0)$ & Ref. & \\
\hline Pre-frail (MFI = 1-2) & $1.25(1.09-1.44)$ & 0.003 \\
\hline Frail $(\mathrm{MFI}>=3)$ & $1.36(1.13-1.65)$ & 0.002 \\
\hline SOFA & $1.03(1.02-1.05)$ & $<0.001$ \\
\hline \multicolumn{3}{|l|}{ SAPS-3 (quartiles) } \\
\hline$\leq 42$ & Ref. & \\
\hline $43-50$ & $1.32(1.12-1.55)$ & 0.002 \\
\hline $51-61$ & $1.62(1.38-1.91)$ & $<0.001$ \\
\hline$>61$ & $1.44(1.18-1.75)$ & $<0.001$ \\
\hline Admission from emergency department & $1.38(1.27-1.5)$ & $<0.001$ \\
\hline Hypertension & $0.8(0.73-0.89)$ & $<0.001$ \\
\hline Diabetes & $1.03(0.95-1.13)$ & 0.437 \\
\hline Obesity & $1.11(0.98-1.26)$ & 0.086 \\
\hline COPD or Asthma & $1.07(0.95-1.2)$ & 0.239 \\
\hline Cardiovascular disease & $1.13(1.02-1.24)$ & 0.016 \\
\hline Vasopressor & $1.31(1.19-1.44)$ & $<0.001$ \\
\hline Renal Replacement Therapy & $1.45(1.33-1.57)$ & $<0.001$ \\
\hline \multicolumn{3}{|l|}{$\mathrm{PaO} 2 / \mathrm{FiO} 2$} \\
\hline Normal (> 300) & Ref. & \\
\hline Mild (201-300) & $1.14(0.89-1.44)$ & 0.251 \\
\hline Moderate (101-200) & $1.12(0.88-1.41)$ & 0.306 \\
\hline Severe $(\leq 100)$ & $1.23(0.93-1.64)$ & 0.123 \\
\hline \multicolumn{3}{|l|}{ Initial respiratory support } \\
\hline $\begin{array}{l}\text { Noninvasive Respiratory Support first } \\
\text { Invasive Mechanical Ventilation first }\end{array}$ & $\begin{array}{l}0.59(0.54-0.65) \\
\text { Ref. }\end{array}$ & $<0.001$ \\
\hline
\end{tabular}

HR - Hazard Ratio; SAPS-3 - Simplified Acute Physiology Score; SOFA- Sequential Organ Failure Assessment; COPD - Chronic Obstructive Pulmonary Disease; ICU - Intensive care unit 


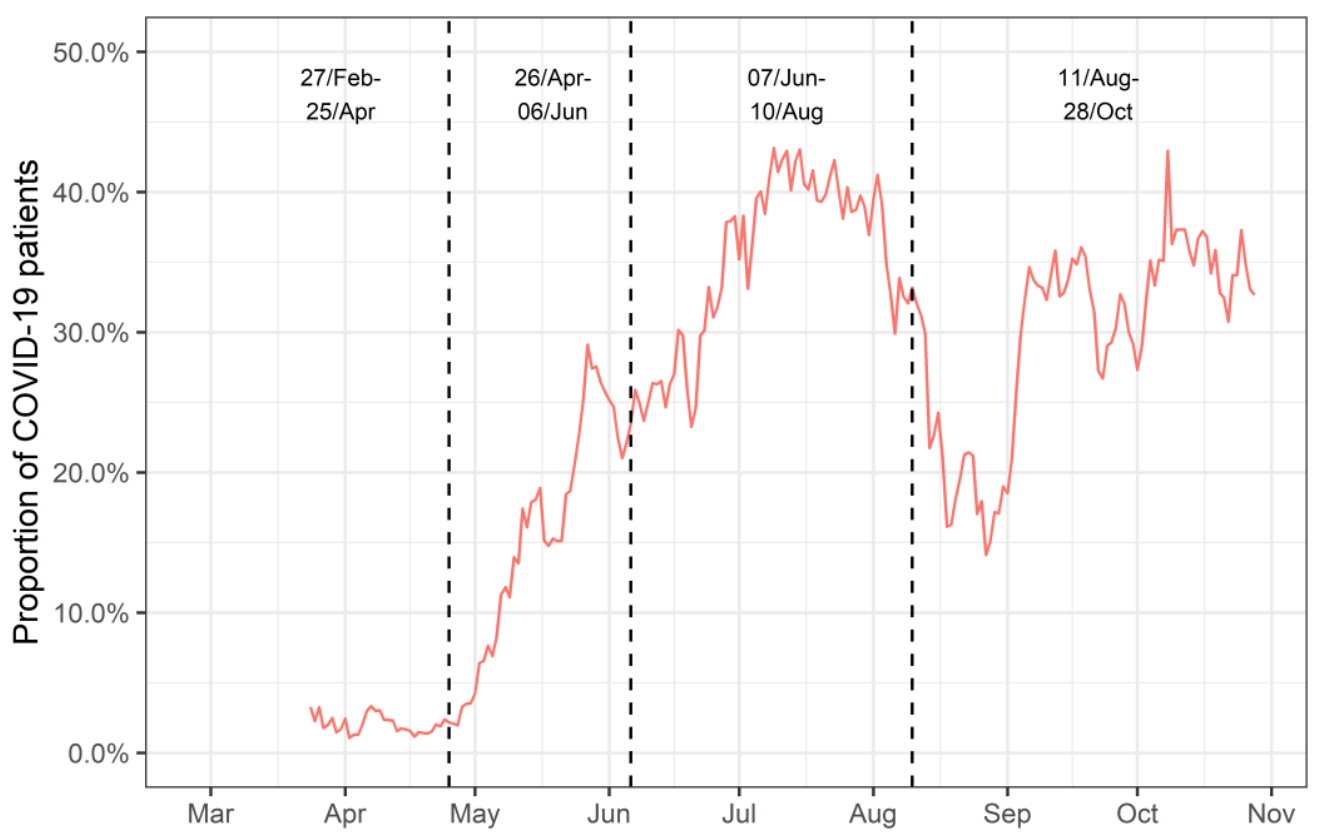

Figure A12.17 - Proportion of COVID-19 patients that underwent steroids administration.

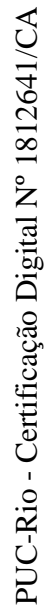

